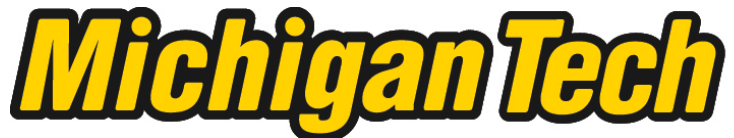 \\ Michigan Technological University Create the Future Digital Commons @ Michigan Tech
}

Development of a 1-D Catalyzed Diesel Particulate Filter Model for Simulation of the Performance and the Oxidation of Particulate Matter and Nitrogen Oxides Using Passive Oxidation and Active Regeneration Engine Experimental Data

Kiran Premchand

Michigan Technological University

Follow this and additional works at: https://digitalcommons.mtu.edu/etds

Part of the Mechanical Engineering Commons

Copyright 2013 Kiran Premchand

Recommended Citation

Premchand, Kiran, "Development of a 1-D Catalyzed Diesel Particulate Filter Model for Simulation of the Performance and the Oxidation of Particulate Matter and Nitrogen Oxides Using Passive Oxidation and Active Regeneration Engine Experimental Data", Dissertation, Michigan Technological University, 2013. https://doi.org/10.37099/mtu.dc.etds/672

Follow this and additional works at: https://digitalcommons.mtu.edu/etds

Part of the Mechanical Engineering Commons 
DEVELOPMENT OF A 1-D CATALYZED DIESEL PARTICULATE FILTER MODEL FOR SIMULATION OF THE PERFORMANCE AND THE OXIDATION OF PARTICULATE MATTER AND NITROGEN OXIDES USING PASSIVE OXIDATION AND ACTIVE REGENERATION ENGINE EXPERIMENTAL DATA

By

Kiran C. Premchand

\begin{abstract}
A DISSERTATION
Submitted in partial fulfillment of the requirements for the degree of DOCTOR OF PHILOSOPHY in Mechanical Engineering - Engineering Mechanics
\end{abstract}

MICHIGAN TECHNOLOGICAL UNIVERSITY

2013

(c) 2013 Kiran C. Premchand 

This dissertation, "Development of a 1-D Catalyzed Diesel Particulate Filter Model for Simulation of the Performance and the Oxidation of Particulate Matter and Nitrogen Oxides Using Passive Oxidation and Active Regeneration Engine Experimental Data," is hereby approved in partial fulfillment for the requirements for the Degree of DOCTOR OF PHILOSOPHY in Mechanical Engineering - Engineering Mechanics.

Department of Mechanical Engineering - Engineering Mechanics

Dissertation Co-Advisor: Dr. John H. Johnson

Dissertation Co-Advisor: Dr. Song-Lin (Jason) Yang

Committee Member: Dr. Jeffrey D. Naber

Committee Member: Dr. Gordon G. Parker

Committee Member: Dr. Jason M. Keith

Department Chair: Dr. William W. Predebon 



\section{CONTENTS}

List of Figures $\ldots \ldots \ldots \ldots \ldots \ldots \ldots \ldots \ldots \ldots \ldots \ldots$

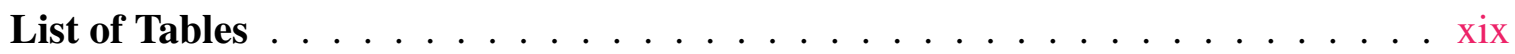

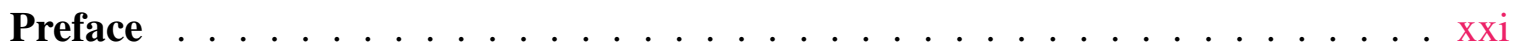

Acknowledgments $\ldots \ldots \ldots \ldots \ldots \ldots \ldots \ldots \ldots \ldots \ldots \ldots \ldots \ldots \ldots$

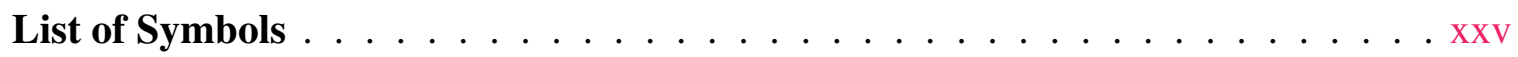

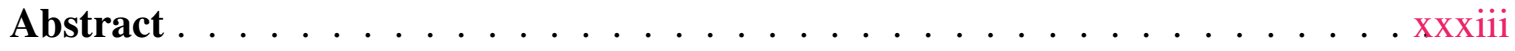

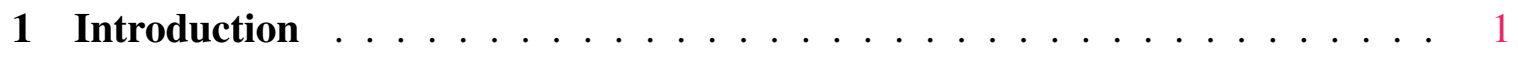

1.1 Research Objectives . . . . . . . . . . . . . . . . . 2

1.2 Overview of Thesis $\ldots \ldots \ldots \ldots \ldots \ldots \ldots$

2 Background and Literature Review $\ldots \ldots \ldots \ldots \ldots$

2.1 1-D CPF Models $\ldots \ldots \ldots \ldots \ldots \ldots \ldots$

2.2 Filtration and Deposition of $\mathrm{PM} \ldots \ldots \ldots \ldots$

2.3 Oxidation of $\mathrm{PM} \ldots \ldots \ldots \ldots \ldots \ldots \ldots \ldots \ldots$ 
2.4 Oxidation of Gaseous Species (Catalytic Reactions) and Back-Diffusion of $\mathrm{NO}_{2} \ldots \ldots \ldots \ldots \ldots \ldots \ldots \ldots \ldots$

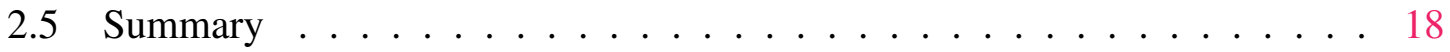

3 Development of the CPF Model . . . . . . . . . . . . . . . . . . . . . . . . . . 19

3.1 Brief Development History . . . . . . . . . . . . . . . . . . . . . . 19

3.2 Overview of the Model . . . . . . . . . . . . . . . . . 21

3.3 Structure of the Model Code . . . . . . . . . . . . . . . . . . 25

3.4 Submodels and Numerical Solution Procedure . . . . . . . . . . . . . 27

3.4.1 Calculating Thermophysical Properties of the Exhaust Gas . . . . 27

3.4.2 Packing Density of PM in the Wall . . . . . . . . . . . 30

3.4.3 Solving the Gaseous Velocity Field . . . . . . . . . . . 31

3.4.4 Solving the Temperature Field . . . . . . . . . . . . . 35

3.4.5 Gaseous Species Concentrations Field - Catalyst Submodel . . . . . 40

3.4.6 PM Mass Balance and PM Oxidation . . . . . . . . . . . 46

3.4.7 Filtration of PM by PM Cake Layer and Substrate Wall . . . . . . 50

3.4.8 Solution Convergence Check . . . . . . . . . . . . . . 56

4 Experimental Data and Model Calibration Procedure . . . . . . . . . . . 58

4.1 Passive Oxidation (PO) Test Matrix $\ldots \ldots \ldots \ldots$

4.2 Active Regeneration (AR) Test Matrix . . . . . . . . . . . . . . . 60

4.3 Procedure for the Model Calibration . . . . . . . . . . . . . . 62

4.3 .1 Filtration $\ldots \ldots \ldots \ldots \ldots \ldots \ldots \ldots \ldots$

4.3.2 Catalytic Reaction Kinetics . . . . . . . . . . . . . . . . . 64

4.3.3 PM Oxidation Kinetics . . . . . . . . . . . . . . . 65 
5 Results and Discussion . . . . . . . . . . . . . . . . . . . . 70

5.1 Input Parameters Obtained from Model Calibration $\ldots \ldots \ldots \ldots$

5.1.1 Filtration Parameters . . . . . . . . . . . . . . . . . . 70

5.1.2 Catalytic Reaction Kinetics . . . . . . . . . . . . . . . . 71

5.1.3 PM Oxidation Kinetics . . . . . . . . . . . . . . . 73

5.2 CPF Model Results from Optimized Kinetics _ . . . . . . . . . 78

5.2.1 Passive Oxidation Case . . . . . . . . . . . . . . . . 78

5.2.2 Active Regeneration Case $\ldots \ldots \ldots$. . . . . . . . 84

5.3 Analysis of PM Mass Oxidized by Mechanism _ . . . . . . . . . . 91

5.4 Analysis of Reaction Rates Obtained from Calibrated PM Oxidation Kinetic Parameters . . . . . . . . . . . . . . . . . . . 92

5.5 Effect of PM Oxidation on PM Cake Layer Thickness . . . . . . . . . . 101

5.6 Effect of Back-Diffusion of $\mathrm{NO}_{2}$ on PM Oxidation Rate $\ldots \ldots . . .105$

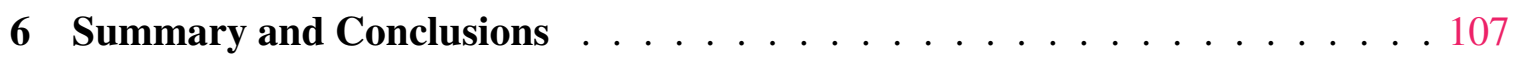

6.1 Summary of CPF Model Development . . . . . . . . . . . . . 107

6.2 Summary of CPF Model Calibration $\ldots \ldots \ldots \ldots \ldots$

6.3 Summary of Major Findings $\ldots \ldots \ldots \ldots \ldots$

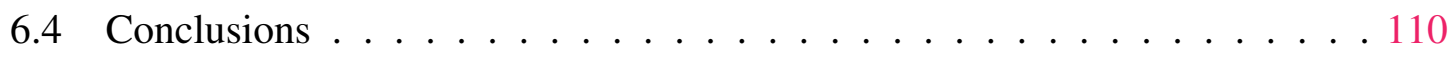

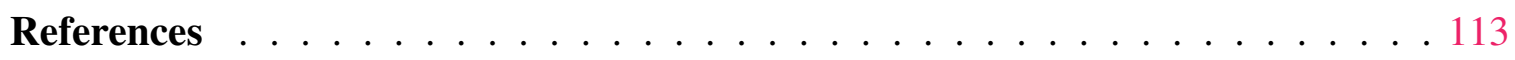

Appendix A Derivation of Conservation of Mass of Exhaust Gas Mixture ․ 120

Appendix B Derivation of Equations for Calculating the Gaseous Velocity Field 124 vii 
Appendix C Derivation of Conservation of Energy Equations $\ldots \ldots \ldots$

Appendix D Derivation of Gaseous Species Concentrations Field $\ldots \ldots$

Appendix E Flowcharts for the CPF Model Source Code . . . . . . . . . . . . 147

Appendix F Compiling and Running the CPF Model Source Code $\ldots \ldots$

Appendix G Gas Property Coefficients Used . . . . . . . . . . . . . . . 158

Appendix H Exhaust Gas Input Data $\ldots \ldots \ldots$

Appendix I Calibration Parameters . . . . . . . . . . . . . . . . . 164

Appendix J Comparison of Model Results to Experimental Data $\ldots \ldots 6$

Appendix K Additional PM Reaction Rate Results from Calibrated PM

Oxidation Kinetic Parameters for the Three Fuels Studied . . . . . . . . . 174

Appendix L Copyright Agreements and Letters of Permission . . . . . . . . 177 


\section{LIST OF FigureS}

2.1 Comparison of thermal contours during a forced regeneration event from experiments and model [17] - Reprinted with permission from SAE Paper No. 2001-01-0912 (c)2001 SAE International. . . . . . . . . . . . . . . . . 7

2.2 Schematic representation of flow of exhaust gas mixture in a wall-flow type particulate filter - adapted from reference [35] . . . . . . . . . . . . 9

2.3 Unit collector filtration efficiency [36] - Reprinted with permission from SAE Paper No. 2007-01-0921 (c)2007 SAE International. . . . . . . . . . . 11

2.4 Measured filtration efficiency evolution for 4 different filters having different filter microstructure and geometry properties. Re-produced from reference $[32] \ldots \ldots \ldots \ldots$. . . . . . . . . . . . . . . . . . . . . . .

2.5 Inlet channel, outlet channel and wall velocity distributions at different Reynolds numbers[37]. - Reprinted with permission from SAE Paper No. 2002-01-1311 (c)2002 SAE International. . . . . . . . . . . . . 13

2.6 Comparison of normalized wall velocity distributions from two different models - 3D model (KIVA3v) and 1D model at the same flow conditions $\left(\right.$ space vel. $\left.=30 \mathrm{~s}^{-1}\right)$ and geometry $(\mathrm{EX}-802.66 " \mathrm{x} 4.5 " 100 / 17$, channel length $=114 \mathrm{~mm}$, channel width $=2.28 \mathrm{~mm}$, wall thickness $=0.432 \mathrm{~mm}$, porosity $=48 \%$, mean pore size $=12 \mu \mathrm{m}$ ) [38]. - Reprinted with permission from SAE Paper No. 2005-01-0963 (c)2005 SAE International. . . . . . . . 14

2.7 Total reaction rates obtained from oxidation of PM derived from ULSD (red squares) and B100 (green circles) fuels[49] - Reprinted with permission from the authors of SAE Paper No. 2010-01-2127 - Appendix L. 16 
2.8 $\mathrm{NO}_{2}$ concentration profiles at three different temperatures near filter entrance $(\mathrm{z} / \mathrm{L}=0.25)[53]$ - Reprinted with permission from SAE Paper No. 2004-01-0696 @ 2004 SAE International. . . . . . . . . . . . . . . . 17

2.9 $\mathrm{NO}_{2}$ concentration profiles at three different temperatures near filter exit $(\mathrm{z} / \mathrm{L}=0.95)$ [53] - Reprinted with permission from SAE Paper No. 2004-01-0696 @2004 SAE International. . . . . . . . . . . . . . . 17

3.1 Schematic representation of the single channel approach used in the development of the CPF model. Reprinted with permission from SAE Paper No. 2013-01-1574 @2013 SAE International. . . . . . . . . . . . 22

3.2 Schematic representation of the discretized inlet channel, PM cake layer, catalyst washcoat, substrate wall and outlet channel control volumes . . . . 23

3.3 Schematic representation of the PM cake layer, catalyst washcoat and substrate wall (cake+cat+wall) control volume discretized in the transverse (y) direction. In this case, number of discretizations in the PM cake layer $=3$, number of discretizations in the catalyst washcoat $=3$, and number of discretizations in the substrate wall $=4$. Reprinted with permission from SAE Paper No. 2013-01-1574 (c)2013 SAE International. . . . . . . . . . . 24

3.4 Flowchart showing the overall structure of the CPF model . . . . . . . . . 26

3.5 Model-predicted CPF pressure drop with constant and variable wall packing density compared to experimental pressure drop . . . . . . . 31

3.6 Diagram showing the calculation of effective inlet channel width . . . . . 32

3.7 Diagram showing an isometric view of the cake+cat+wall control volume with the geometric dimensions as well as interface areas . . . . . . . 37

3.8 Schematic representation of two different types of catalyst location (a. and b.), model framework (c.) and model setup for both catalyst location types when simulating (d. and e.) . . . . . . . . . . . . . . 43

3.9 Schematic representation of cake+cat+wall control volume, showing the different domains and the chemical reactions associated with them reactions are as given in Table $3.2 \ldots \ldots \ldots$. . . . . . . . . . 44

3.10 Numerical discretizations in the axial (x) and transverse (y) directions in the substrate wall sub-domain of the CPF model . . . . . . . . . . . 48 
3.11 Schematic showing catalyst sub-model domain grid: (a) before the addition of the virtual wall PM layer, and (b) after the addition of the virtual wall PM layer . . . . . . . . . . . . . . . . . . . . 49

3.12 Schematic of filtration efficiency and outlet mass calculations . . . . . . 50

3.13 Diagram of unit cell in substrate wall . . . . . . . . . . . . 51

3.14 Schematic of streamlines (shown as dotted lines) for calculating pressure drop across CPF $($ Here,$j=4) \ldots \ldots . \ldots . \ldots 55$

4.1 Overview of the various stages of the typical passive oxidation experiment - adapted from reference [54] . . . . . . . . . . . . . . . 60

4.2 Overview of the various stages of the typical active regeneration experiment - adapted from reference [55] . . . . . . . . . . . . 61

5.1 Arrhenius plot of $\mathrm{NO}_{2}$-assisted PM oxidation reaction in PM cake layer shown for all passive oxidation experiments . . . . . . . . . . . . 74

5.2 Arrhenius plot of $\mathrm{NO}_{2}$-assisted PM oxidation reaction in substrate wall shown for all passive oxidation experiments . . . . . . . . . 75

5.3 Arrhenius plot of thermal $\left(\mathrm{O}_{2}\right)$ PM oxidation reaction in PM cake layer shown for all active regeneration experiments . . . . . . . . . . . 76

5.4 Arrhenius plot of thermal $\left(\mathrm{O}_{2}\right)$ PM oxidation reaction in substrate wall shown for all active regeneration experiments . . . . . . . . . . . 77

5.5 Comparison of experimental and model total pressure drop across CPF and its components - PO-B20-13 . . . . . . . . . . . . . . . 79

5.6 Model cumulative PM mass balance and comparison of experimental and model PM mass retained - PO-B20-13 (Table in the inset shows a comparison of experimental and model PM mass retained at the end of

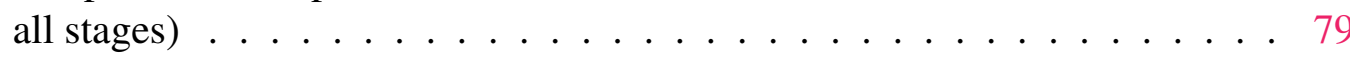

5.7 Distribution of model total PM mass retained into PM cake and substrate wall - PO-B20-13 . . . . . . . . . . . . . . . . 80

5.8 Distribution of total PM oxidation rate (top) and cumulative PM mass oxidized (bottom) into thermal (red) and $\mathrm{NO}_{2}$-assisted (blue) mechanisms . 81 
5.9 Distribution of total PM oxidation rate (top) and cumulative PM mass oxidized (bottom) into PM cake (purple) and substrate wall (green) PO-B20-13 ........................ 81

5.10 Comparison of experimental and model total filtration efficiency and distribution of filtration efficiency into PM cake and substrate wall PO-B20-13 ......................... 82

5.11 Comparison of experimental and model CPF outlet gas temperature with model input CPF inlet gas temperature - PO-B20-13 [Top left: comparison of temperatures for 12 minutes during the start of passive oxidation stage, Top right: comparison of temperatures for 12 minutes during the end of passive oxidation stage, Bottom: comparison of temperatures for the entire duration of the experiment] . . . . . . . . . . . . . . 83

5.12 Comparison of experimental and model CPF outlet $\mathrm{NO}_{2}$ concentrations with model input CPF inlet $\mathrm{NO}_{2}$ concentrations - PO-B20-13 . . . . . . . . 84

5.13 Comparison of experimental and model total pressure drop across CPF and its components - AR-B10-1 . . . . . . . . . . . . . 85

5.14 Model cumulative PM mass balance and comparison of experimental and model PM mass retained - AR-B10-1 (Table in the inset shows a comparison of experimental and model PM mass retained at the end of all stages $\ldots \ldots \ldots \ldots \ldots$. . . . . . . . . . . . . . 86

5.15 Distribution of model total PM mass retained into PM cake and substrate wall - AR-B10-1 . . . . . . . . . . . . . . . 86

5.16 Distribution of total PM oxidation rate (top) and total cumulative PM mass oxidized (bottom) into thermal (red) and $\mathrm{NO}_{2}$-assisted (blue) mechanisms - AR-B10-1 . . . . . . . . . . . . . . . . 87

5.17 Distribution of total PM oxidation rate (top) and total cumulative PM mass oxidized (bottom) into PM cake (purple) and substrate wall (green) AR-B10-1 . . . . . . . . . . . . . . . . 88

5.18 Comparison of experimental and model total filtration efficiency and distribution of filtration efficiency into filtration efficiency of PM cake and filtration efficiency of substrate wall - AR-B10-1 . . . . . . . . . . . 88

5.19 Comparison of experimental and model $\mathrm{CPF}$ outlet gas temperature with model input CPF inlet gas temperature - AR-B10-1 . . . . . . . . . . . 89 
5.20 Comparison of experimental and model CPF outlet $\mathrm{NO}_{2}$ concentrations with model input CPF inlet $\mathrm{NO}_{2}$ concentrations - AR-B10-1 . . . . . . 90

5.21 Fraction of total PM mass oxidized by thermal and $\mathrm{NO}_{2}$-assisted PM oxidation reactions plotted against average $\mathrm{CPF}$ temperature during passive oxidation and active regeneration stages of all experiments considered for CPF model calibration . . . . . . . . . . . . . . . . . . . . . . . . 91

5.22 PM oxidation reaction rates (plotted in log-scale) showing $\mathrm{NO}_{2}$-assisted reaction rates at 5 different $\mathrm{NO}_{2}$ concentrations with thermal $\left(\mathrm{O}_{2}\right)$ reaction (black line) rate shown as functions of temperature for ULSD fuel-type . . 93

5.23 PM oxidation reaction rates (plotted in log-scale) showing $\mathrm{NO}_{2}$-assisted reaction rates at 5 different $\mathrm{NO}_{2}$ concentrations with thermal $\left(\mathrm{O}_{2}\right)$ reaction (black line) rate shown as functions of temperature for B10 fuel-type . . . 93

5.24 PM oxidation reaction rates (plotted in log-scale) showing $\mathrm{NO}_{2}$-assisted reaction rates at 5 different $\mathrm{NO}_{2}$ concentrations with thermal $\left(\mathrm{O}_{2}\right)$ reaction (black line) rate shown as functions of temperature for B20 fuel-type . . . 94

5.25 Comparison of $\mathrm{NO}_{2}$-assisted (blue), thermal (red) and total (black) shown for 3 different $\mathrm{NO}_{2}$ concentrations (top $=10 \mathrm{ppm}$, middle $=50 \mathrm{ppm}$ and bottom $=150 \mathrm{ppm}$ ) as a function of increasing temperature for ULSD

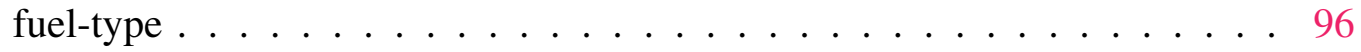

5.26 Comparison of $\mathrm{NO}_{2}$-assisted (blue), thermal (red) and total (black) shown for 3 different $\mathrm{NO}_{2}$ concentrations (top $=10 \mathrm{ppm}$, middle $=50 \mathrm{ppm}$ and bottom $=150 \mathrm{ppm}$ ) as a function of increasing temperature for B10 fuel-type 97

5.27 Comparison of $\mathrm{NO}_{2}$-assisted (blue), thermal (red) and total (black) shown for 3 different $\mathrm{NO}_{2}$ concentrations (top $=10 \mathrm{ppm}$, middle $=50 \mathrm{ppm}$ and bottom $=150 \mathrm{ppm}$ ) as a function of increasing temperature for B20 fuel-type 98

5.28 Plot showing lines of equal total reaction rates (green) from calibrated PM kinetics obtained and lines of equal fraction of $\mathrm{NO}_{2}$-assisted to total PM oxidation reaction rate (blue) for the ULSD fuel type as functions of increasing $\mathrm{NO}_{2}$ concentrations along $\mathrm{x}$ axis and increasing temperatures along $\mathrm{y}$ axis. The red ' $\mathrm{x}$ ' shows one active regeneration experiment (AR-ULSD-2: $\mathrm{NO}_{2}=7 \mathrm{ppm}$, Temp. $=581{ }^{\circ} \mathrm{C}$, total reaction rate from calibrated model $\left.=2.3 \times 10^{-03} \mathrm{~s}^{-1}\right) \ldots \ldots \ldots \ldots 9 . \ldots \ldots$ 
5.29 Plot showing lines of equal total reaction rates (green) from calibrated PM kinetics obtained and lines of equal fraction of $\mathrm{NO}_{2}$-assisted to total PM oxidation reaction rate (blue) for the B10 fuel type as functions of increasing $\mathrm{NO}_{2}$ concentrations along $\mathrm{x}$ axis and increasing temperatures along $\mathrm{y}$ axis. The red ' $\mathrm{x}$ ' shows one active regeneration experiment (AR-B10-4: $\mathrm{NO}_{2}=3 \mathrm{ppm}$, Temp. $=554{ }^{\circ} \mathrm{C}$, total reaction rate from calibrated model $\left.=1.5 \times 10^{-03} \mathrm{~s}^{-1}\right) \ldots \ldots \ldots$. . . . . . . 99

5.30 Plot showing lines of equal total reaction rates (green) from calibrated PM kinetics obtained and lines of equal fraction of $\mathrm{NO}_{2}$-assisted to total PM oxidation reaction rate (blue) for the B20 fuel type as functions of increasing $\mathrm{NO}_{2}$ concentrations along $\mathrm{x}$ axis and increasing temperatures along $\mathrm{y}$ axis. The red ' $\mathrm{x}$ ' shows one active regeneration experiment (AR-B20-6: $N O_{2}=10 \mathrm{ppm}$, Temp. $=531{ }^{\circ} \mathrm{C}$, total reaction rate from calibrated model $\left.=1.3 \times 10^{-03} \mathrm{~s}^{-1}\right) \ldots \ldots \ldots \ldots$

5.31 Plots showing the variation of PM cake layer thickness along axial (x) direction and time, with smaller plots showing: (lower left) PM cake thickness profiles at 3 different time points and (lower right) the cake thickness at 3 different axial locations - PO-B20-12 . . . . . . . . 101

5.32 Plots showing the variation of PM cake layer thickness along axial (x) direction and time, with smaller plots showing: (lower left) the cake thickness profiles at 3 different time points and (lower right) the cake thickness at 3 different axial locations - AR-ULSD-1 . . . . . . . 102

5.33 Axial PM loading distribution from two sets of experimental data [39] compared to a similar point in the simulation of AR-B10-1 at the end of loading stage $2 \ldots \ldots \ldots \ldots \ldots$

5.34 Axial PM loading distribution from experimental data [39] at the end of passive oxidation compared to model results at a similar point in the simulation of PO-B20-12 . . . . . . . . . . . . . . . . . 104

5.35 Axial PM loading distribution from experimental data [39] at the end of active regeneration compared to model results at a similar point in the simulation of AR-ULSD-1 . . . . . . . . . . . . . . . . 104

A.1 Diagram showing dimensions of inlet channel control volume and components of mass balance in inlet channel control volume . . . . . . 120 
A.2 Diagram showing dimensions of the cake+cat+wall control volume and components of mass balance in the cake+cat+wall control volume . . . . 121

A.3 Diagram showing dimensions of outlet channel control volume and components of mass balance in outlet channel control volume . . . . . . 123

C.1 Schematic representation of a control volume in the inlet channel of the CPF 133

C.2 Schematic representation of a control volume in the outlet channel of the CPF . . . . . . . . . . . . . . . . . . . 134

D.1 Schematic representation of the PM cake, catalyst washcoat and substrate wall control volume . . . . . . . . . . . . . . . . . . . 136

D.2 Sample discretization of the PM cake, catalyst washcoat and substrate wall control volume (Here, $n_{\text {div,cake }}=3, n_{\text {div, cat }}=2$ and $n_{\text {div, wall }}=3$ ). Also shown is the wall PM control volume with the calculated virtual wall layer thickness equal to $w_{\text {wall }} \ldots \ldots \ldots \ldots$. . . . . . . . . 137

D.3 Schematic representation of an interior node and its neighboring nodes . . 138

D.4 Schematic representation of an interface shown between two nodes that are in different materials . . . . . . . . . . . . . . . . . . 144

E.1 Flowchart showing a detailed flow of logic in the MTU CPF high-fidelity model version 4.0. Reprinted with permission from SAE Paper No. 2013-01-1574 (C2013 SAE International. . . . . . . . . . . . . . . . 148

E.2 Flowchart of the Startup subroutine as shown in Figure E.1 . . . . . . 149

E.3 Flowchart of the Read Inputs subroutine as shown in Figure E.1 _ . . . 150

E.4 Flowchart of the Set Initial Conditions subroutine as shown in Figure E.1 . 151

E.5 Flowchart of the Inlet Interpolation subroutine as shown in Figure E. 1 . . 152

E.6 Flowchart of the filtration subroutine as shown in Figure E.1 - box titled 'Filtration (cake+wall)' . . . . . . . . . . . . . . . . . 153 
H.1 AR-B20-1 - Corrected and Experimental CPF Flow Resistance (subplot 1), Exhaust Viscosity and CPF Average Temperature (subplot 2), Corrected and Corrected Experimental Exhaust Mass Flow Rate (subplot 3), Corrected and Experimental CPF Pressure Drop Profile (subplot 4) . . . . . 161

I.1 PM cake permeability values used for calibration of passive oxidation cases 164

K.1 Thermal to total and $\mathrm{NO}_{2}$-assisted to total reaction rate percentages shown for 3 different fuel types $-\mathrm{O}_{2}=9 \%$ Vol. and $\mathrm{NO}_{2}=50 \mathrm{ppm}$. Results for $\mathrm{O}_{2}=9 \%$ Vol. and $\mathrm{NO}_{2}=100 \mathrm{ppm}$ and 150 ppm are shown in Table K.1 . . 174

K.2 Total reaction rates along y axis (shown in log-scale) versus temperature for 3 different fuel types $-\mathrm{O}_{2}=9 \%$ Vol. and $\mathrm{NO}_{2}=50 \mathrm{ppm}$. Results for $\mathrm{O}_{2}=9 \%$ Vol. and $\mathrm{NO}_{2}=100$ ppm and 150 ppm are shown in Table K.1 . . 175 


\section{LIST OF TABLES}

2.1 Activation energies of PM oxidation reported in open literature . . . . . . 15

3.1 Tabulated list of chemical species considered in the CPF model version 4.0 41

3.2 Chemical reactions considered in the CPF model. Here, $i=$ cake, wall . . . 43

4.1 Specifications of the after-treatment system used in the experiments [4,61] . 58

4.2 Specifications of the different fuels used in the experiments [54,55] . . . . 59

4.3 Passive oxidation experiments used for the calibration of the CPF model . . 60

4.4 Active regeneration experiments used for the calibration of the CPF model . 62

4.5 Filtration-related input parameters considered for the CPF model calibration 67

4.6 Catalytic reaction kinetic parameters considered for the CPF model

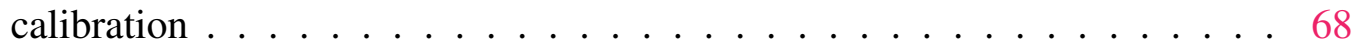

4.7 PM oxidation kinetic parameters considered for the CPF model calibration . 69

4.8 Initial values of kinetic parameters of PM oxidation reactions determined by optimization computation of $\mathrm{NO}_{2}$-assisted PM oxidation and from analysis of experimental data in reference [4] used for calibration of high-fidelity CPF model . . . . . . . . . . . . . . . 69

5.1 Single set of filtration parameters obtained from CPF model calibration . . 72

5.2 Single set of kinetic parameters for catalytic reactions obtained from $\mathrm{CPF}$ model calibration . . . . . . . . . . . . . . . . 73 xvii 
5.3 Single set of kinetic parameters of PM oxidation obtained from CPF model

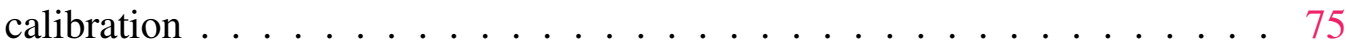

5.4 Effect of back-diffusion of $\mathrm{NO}_{2}$ on PM oxidation for all passive oxidation

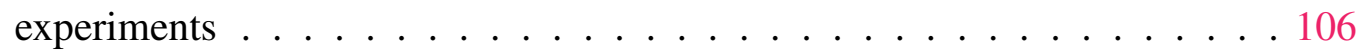

G.1 Coefficients used in the calculation of specific heat of exhaust gas mixture - see equation (3.4) [66] . . . . . . . . . . . . . . . . . 158

G.2 Coefficients used in the calculation of dynamic viscosity of individual chemical species - see equation (3.6) [66] . . . . . . . . . . 158

G.3 Coefficients used in the calculation of thermal conductivity of individual chemical species - see equation (3.10) [66] . . . . . . . . . . . . 159

H.1 Correction factors (CF) used for calibration of the CPF model $\ldots \ldots$

H.2 Original and modified values of $\mathrm{CPF}$ inlet PM concentrations and $\mathrm{CPF}$ inlet NO2/NOx for all experiments used for calibration of CPF model . . . . . 163

I.1 Filtration parameters for all test cases used for calibration of CPF model . 165

J.1 Comparison of experimental and model total pressure drop across the CPF at three different time points during each experiment. Model results are from simulations using filtration parameters in Table I.1, catalytic reaction kinetics from Table 5.2 and PM oxidation kinetics from Table 5.3 . . . . 166

J.2 Comparison of experimental and model total PM mass retained in the CPF at the end of all stages. Model results are from simulations using filtration parameters in Table I.1, catalytic reaction kinetics from Table 5.2 and PM oxidation kinetics from Table 5.3 . . . . . . . . . . . . . . . 167

J.3 Comparison of experimental and model CPF total filtration efficiency during Stage-2 loading. Model results are from simulations using filtration parameters in Table I.1, catalytic reaction kinetics from Table 5.2 and PM oxidation kinetics from Table 5.3 . . . . . . . . . . . . . . . 168

J.4 Comparison of experimental and model CPF outlet temperature during $\mathrm{PO} / \mathrm{AR}$. Model results are from simulations using filtration parameters in Table I.1, catalytic reaction kinetics from Table 5.2 and PM oxidation kinetics from Table 5.3 . . . . . . . . . . . . . . . . . . . 169 
J.5 Comparison of experimental and model CPF outlet $\mathrm{NO}_{2}$ concentrations averaged during Loading, PO/AR and Post-loading. Model results are from simulations using filtration parameters in Table I.1, catalytic reaction kinetics from Table 5.2 and PM oxidation kinetics from Table 5.3 . . . . 170

J.6 Comparison of experimental and model total pressure drop across the CPF at three different time points during each experiment. Model results are from simulations using a single set of input parameters shown in Tables $5.1,5.2$ and $5.3 \ldots \ldots \ldots \ldots \ldots \ldots$

J.7 Comparison of experimental and model total PM mass retained in the CPF at the end of all stages. Model results are from simulations using a single set of input parameters shown in Tables 5.1, 5.2 and 5.3 . . . . . . . 172

J.8 Comparison of experimental and model CPF total filtration efficiency during Stage-2 loading. Model results are from simulations using a single set of input parameters shown in Tables 5.1, 5.2 and $5.3 \ldots \ldots . . . .173$

K.1 Total reaction rates calculated from the calibrated PM oxidation kinetic parameters shown in Table 5.3 for the three different fuels along with percentages of thermal to total and $\mathrm{NO}_{2}$-assisted to total fractions shown for each case . . . . . . . . . . . . . . . . . . 176 



\section{PREFACE}

The work presented in this $\mathrm{PhD}$ dissertation has been published or is currently under consideration for publication by SAE International as two technical papers.

The first paper covers the development and description of the numerical model developed in this work, and is presented in parts of Chapters 1, 2, 3 and 4 of this dissertation. This paper was titled "Development of a 1-D Catalyzed Diesel Particulate Filter Model for Simulation of the Oxidation of Particulate Matter and Gaseous Species during Passive Oxidation and Active Regeneration" and was published and presented at the 2013 SAE World Congress held on April 16-18 at the Cobo Center in Detroit, Michigan. The authors of this publication are Kiran C. Premchand, Dr. John H. Johnson and Dr. Song-Lin (Jason) Yang, all of Michigan Technological University. Kiran Premchand was responsible for the development of the model. Dr. John Johnson provided technical guidance regarding the experimental data and modeling aspects. Dr. Song-Lin Yang provided technical guidance with the numerical aspects of model development.

The second paper explains the development of a calibration procedure for the numerical model based on engine experimental data and the application of this calibration procedure to determine input parameters to the model and is presented in parts of Chapters 4, 5 and 6 of this dissertation. This paper is tentatively titled "Particulate Matter and Nitrogen Oxides Kinetics Based on Engine Experimental Data for a Catalyzed Diesel Particulate Filter" and is planned to be presented at the 2014 SAE World Congress on April 8-10, 2014 at the Cobo Center in Detroit, Michigan. The authors of this publication are Kiran C. Premchand, Dr. Harsha S. Surenahalli and Dr. John H. Johnson, all of Michigan Technological University. Kiran Premchand was responsible for the development of the model calibration procedure and the calibration of the model according to this procedure. Dr. Harsha Surenahalli provided technical assistance with numerical optimization. Dr. John Johnson provided technical guidance regarding the experimental data and modeling aspects. The manuscript is currently being reviewed by SAE International. 



\section{ACKNOWLEDGMENTS}

This research work was made possible by the support of several persons and organizations. I would like to sincerely thank all of them for the opportunity.

I would like to thank Dr. John Johnson for being my advisor and Dr. Jason Yang for being my co-advisor. I would like to thank them both for all the help and advice that they have provided me during the course of my degree work. I would also like to thank Dr. Jeff Naber, Dr. Gordon Parker and Dr. Jason Keith for being on my graduate defense committee and providing valuable advice during various stages of my research work. I would also like to thank the opportunity to be a part of the Diesel Group at Michigan Tech.

I would like to thank John Deere and the United States Department of Energy for financial support for my graduate research. This research work was initiated in 2006 and was financially supported by John Deere from 2006 to 2010. I would also like to thank Kirby Baumgard, Robert Iverson and Darren Cruchelow of John Deere for their technical assistance in collecting experimental data and answering my questions. From 2010, this research work was supported by the United States Department of Energy under the project titled "Experimental Studies for CPF and SCR Model, Control System, and OBD Development for Engines Using Diesel and Biodiesel Fuels". "This material is based upon work supported by the Department of Energy National Energy Technology Laboratory under Award Number(s) DE-EE0000204". The writing of this thesis was supported by the MTU Diesel Engine Aftertreament Consortium.

I thank the faculty and staff at the Mechanical Engineering - Engineering Mechanics department for their help on a regular basis. I thank the IT department at MTU for their help in providing the computer programs that I needed for my research work as well as for writing this thesis. I sincerely acknowledge the MTU Graduate School for its assistance with thesis submission and defense preparation.

I would also like to extend my gratitude to fellow researchers and graduate students for their help and support in various aspects of my school life. The following are the people that I would like to acknowledge by name: Hasan Mohammed, Harsha Surenahalli, Ryan Foley, Ken Shiel and James Pidgeon. I would also like to thank my family and dear friends 
for all the love and support that they have shown me over the years. I dedicate this thesis to my father, the late KrishnanNair Premchand.

DISCLAIMER: "This report was prepared as an account of work sponsored by an agency of the United States Government. Neither the United States Government nor any agency thereof, nor any of their employees, makes any warranty, express or implied, or assumes any legal liability or responsibility for the accuracy, completeness, or usefulness of any information, apparatus, product, or process disclosed, or represents that its use would not infringe privately owned rights. Reference herein to any specific commercial product, process, or service by trade name, trademark, manufacturer, or otherwise does not necessarily constitute or imply its endorsement, recommendation, or favoring by the United States Government or any agency thereof. The views and opinions of authors expressed herein do not necessarily state or reflect those of the United States Government or any agency thereof." 


\section{LIST OF SYMBOLS}

$\alpha \quad$ Instantaneous solidosity of the unit cell, $=1-\epsilon[$.$] , see equation (3.58)$

$\Delta P_{\text {cake }} \quad$ Pressure drop across the PM cake layer, $[P a]$, see equation (3.66)

$\Delta P_{\text {channels }}$ Pressure drop due to frictional losses in the inlet and outlet channels, $[P a]$, see equation (3.66)

$\Delta P_{\text {total }} \quad$ Total pressure drop across the CPF, $[\mathrm{Pa}]$, see equation (3.66)

$\Delta P_{\text {wall }} \quad$ Pressure drop across the substrate wall, $[P a]$, see equation (3.66)

$\Delta x \quad$ Discretized dimension of a control volume in the $x$ direction of the particulate filter, $[m]$, page 121

$\Delta y_{i} \quad$ Thickness of the $i^{\text {th }}$ wall slab, $[m]$, see equation (3.61)

$\delta y_{m^{+}} \quad$ Distance of separation of node ' $m$ ' from 'east' $\left(m+\frac{1}{2}\right)$ control surface, $[m]$, page 133

$\delta y_{m^{-}} \quad$ Distance of separation of node ' $m$ ' from 'west' $\left(m-\frac{1}{2}\right)$ control surface, $[m]$ , page 133

$\dot{m} \quad$ Mass flow rate into the CPF, $\left[\frac{\mathrm{kg}}{\mathrm{s}}\right]$, see equation (B.9)

$\dot{m} \quad$ Mass flow rate into/out of the control volume, $\left[\frac{\mathrm{kg}}{\mathrm{s}}\right]$, see equation (C.1)

$\dot{Q} \quad$ Heat transfer rate to/from the control volume across the control surfaces of the control volume, $[W]$, see equation (C.1)

$\dot{q}_{\text {cond,cake }} \quad$ Rate of energy transferred between adjacent PM cake layer elements via conduction, $[W]$, see equation (3.28)

$\dot{q}_{\text {cond,wall }}$ Rate of energy transferred between adjacent substrate wall elements via conduction, $[W]$, see equation (3.28)

$\dot{q}_{c o n v, 1-w} \quad$ Rate of energy transferred via convection between inlet channel and substrate wall, $[W]$, see equation (3.28)

$\dot{q}_{c o n v, 2-w} \quad$ Rate of energy transferred via convection between outlet channel and substrate wall, $[W]$, see equation (3.28)

$\dot{q}_{\text {rxn }} \quad$ Resultant energy release rate due to chemical reactions in the cake+cat+wall control volume, $[W]$, see equation (3.28)

$\dot{W} \quad$ Rate of work done on/by the system, $[W]$, see equation (C.1) 
$\epsilon \quad$ Instantaneous porosity of the unit cell, [.] , see equation (3.58)

$\epsilon \quad$ Porosity of porous medium, [.], see equation (D.5)

$\epsilon_{0} \quad$ Clean wall porosity, [.] , see equation (3.55)

$\eta_{\text {cake }} \quad$ Particulate filtration efficiency of PM cake layer, [.] , see equation (3.65)

$\eta_{D R} \quad$ Equivalent collection efficiency of a single wall collector due to diffusion and direct interception, [.], see equation (3.60)

$\eta_{D} \quad$ Collection efficiency of a unit collector in the substrate wall due to Brownian diffusion mechanism, [.], see equation (3.58)

$\eta_{R} \quad$ Particle collection efficiency of a single unit collector due to interception, [.] , see equation (3.59)

$\eta_{s l a b, i} \quad$ PM filtration efficiency of the $i^{t h}$ wall slab, [.], see equation (3.61)

$\frac{\eta_{c}}{d_{c, c a k e}} \quad$ Ratio of filtration efficiency to diameter of PM cake layer collector parameter, $\left[\frac{1}{m}\right]$, see equation (3.65)

$\hat{h} \quad$ Specific enthalpy of the fluid in the control volume, $=\int_{P} c_{p} d T\left[\frac{J}{k g}\right]$, see equation (C.1)

$\hat{u} \quad$ Specific internal energy of the fluid in the control volume, $=\int_{V} c_{v} d T\left[\frac{J}{k g}\right]$, see equation (C.1)

$\lambda \quad$ Mean free path of the gas, $=\frac{\mu}{P} \sqrt{\frac{\pi \bar{R} T}{2 M W_{e x h}}}[m]$, see equation (3.58)

$\lambda_{p} \quad$ Thermal conductivity of the PM cake layer, $\left[\frac{W}{m \cdot K}\right]$, see equation (3.28)

$\lambda_{s} \quad$ Thermal conductivity of the substrate wall, $\left[\frac{W}{m . K}\right]$, see equation (3.28)

$\left(\bar{\rho}_{\text {mix }}\right)_{w} \quad$ Molecular density of exhaust gas mixture in cake+cat+wall control volume, $\left[\frac{\mathrm{kmol} \mathrm{exh}_{\text {exh }}}{\mathrm{m}^{3}}\right]$, see equation (3.35)

$(M W)_{e x h, 1}$ Equivalent molecular weight of the exhaust gas mixture in the inlet channel control volume, $\left[\frac{\mathrm{kg}}{\mathrm{kmol}}\right]$, see equation (3.1)

$(M W)_{e x h, 2}$ Equivalent molecular weight of the exhaust gas mixture in the inlet channel control volume, $\left[\frac{\mathrm{kg}}{\mathrm{kmol}}\right]$, see equation (3.1)

$(M W)_{e x h, w}$ Equivalent molecular weight of the exhaust gas mixture in the inlet channel control volume, $\left[\frac{\mathrm{kg}}{\mathrm{kmol}}\right]$, see equation (3.1)

$\left(P_{1}\right)_{x=0} \quad$ Absolute pressure at CPF inlet, $[P a]$, page 53

$\left(P_{2}\right)_{x=L} \quad$ Absolute pressure at $\mathrm{CPF}$ outlet, $[\mathrm{Pa}]$, page 53

$\left[\mathrm{C}_{12} \mathrm{H}_{24}\right]$ Concentration of $\mathrm{C}_{12} \mathrm{H}_{24},\left[\frac{\mathrm{kmol}}{\mathrm{m}^{3}}\right]$, see equation (3.37)

$[\mathrm{CO}] \quad$ Concentration of $\mathrm{CO},\left[\frac{\mathrm{kmol}}{\mathrm{m}^{3}}\right]$, see equation (3.37)

[NO] Concentration of $N O,\left[\frac{\mathrm{kmol}}{\mathrm{m}^{3}}\right]$, see equation (3.37)

$\left[\mathrm{NO}_{2}\right] \quad$ Concentration of $\mathrm{NO}_{2},\left[\frac{\mathrm{kmol}}{\mathrm{m}^{3}}\right]$, see equation (3.37)

$\left[\mathrm{O}_{2}\right] \quad$ Concentration of $\mathrm{O}_{2},\left[\frac{\mathrm{kmol}}{\mathrm{m}^{3}}\right]$, see equation (3.37)

$\mu \quad$ Dynamic viscosity, $\left[\frac{\mathrm{Ns}}{\mathrm{m}^{2}}\right]$ or $\left[\frac{\mathrm{kg}}{\mathrm{ms}}\right]$, see equation (3.58) 
$\mu_{1} \quad$ Dynamic viscosity of the exhaust gas mixture in the inlet channel of the particulate filter, $\left[\frac{\mathrm{Ns}}{\mathrm{m}^{2}}\right]$, see equation (B.13)

$\mu_{2} \quad$ Dynamic viscosity of the exhaust gas mixture in the outlet channel of the particulate filter, $\left[\frac{\mathrm{Ns}}{\mathrm{m}^{2}}\right]$, see equation (B.13)

$\mu_{i} \quad$ Dynamic viscosity of chemical species $i,\left[\frac{\mathrm{Ns}}{\mathrm{m}^{2}}\right]$ or $\left[\frac{\mathrm{kg}}{\mathrm{ms}}\right]$, see equation (3.5)

$\mu_{m i x} \quad$ Dynamic viscosity of the exhaust gas mixture, $\left[\frac{\mathrm{Ns}}{\mathrm{m}^{2}}\right]$ or $\left[\frac{\mathrm{kg}}{\mathrm{ms}}\right]$, see equation (3.5)

$\mu_{w} \quad$ Dynamic viscosity of the exhaust gas mixture in the cake+cat+wall control volume of the particulate filter, $\left[\frac{N s}{m^{2}}\right]$, see equation (B.14)

$\bar{a}_{c a t} \quad$ Midpoint width of the catalyst washcoat control volume, $=\frac{a+a_{c p}}{2}[m]$, see equation (3.28)

$\bar{a}_{p} \quad$ Midpoint width of the PM cake layer control volume, $=\frac{a_{c p}+a^{*}}{2}[m]$, see equation (3.28)

$\bar{R} \quad$ Universal gas constant, $=8314.4\left[\frac{\mathrm{J}}{\mathrm{kmol.K}}\right]$, see equation (3.58)

$\rho_{1} \quad$ Density of exhaust gas mixture in the inlet channel control volume, $\left[\frac{\mathrm{kg}}{\mathrm{m}^{3}}\right]$, see equation (B.1)

$\rho_{2} \quad$ Density of exhaust gas mixture in the outlet channel control volume, $\left[\frac{\mathrm{kg}}{\mathrm{m}^{3}}\right]$, see equation (B.1)

$\rho_{p w} \quad$ Wall PM packing density, $\left[\frac{\mathrm{kg}}{\mathrm{m}^{3}}\right]$, see equation (3.12)

$\rho_{s} \quad$ Bulk density of substrate wall, $\left[\frac{\mathrm{kg}}{\mathrm{m}^{3}}\right]$, see equation (3.28)

$\rho_{w} \quad$ Density of exhaust gas mixture in the cake+cat+wall control volume, $\left[\frac{\mathrm{kg}}{\mathrm{m}^{3}}\right]$, see equation (B.1)

$\tau \quad$ Tortuosity of porous medium, [.] , see equation (D.5)

$\xi_{i j} \quad$ Stoichiometric coefficient of species $i$ in reaction $j$ (positive values for reactants, negative values for products), [.], see equation (3.35)

a Width of a clean outlet channel of the particulate filter, $[m]$, see equation (B.1)

$a^{*} \quad$ Effective width of an inlet channel of the particulate filter loaded with PM, $=a-2 w_{c a t}-2 w_{p}[m]$, see equation (B.1)

$A_{\eta} \quad$ Maximum PM cake layer filtration efficiency parameter, [.], see equation (3.65)

$A_{C O} \quad$ Pre-exponential factor of the $\mathrm{CO}$ oxidation reaction, $\left[\frac{m^{3}}{k m o l . s}\right]$, see equation (3.37)

$a_{c p} \quad$ Width available to flow at the PM cake layer - catalyst washcoat interface, $=a-2 w_{\text {cat }}[m]$, see equation (3.28)

$A_{H C} \quad$ Pre-exponential factor of the $\mathrm{HC}$ oxidation reaction, $\left[\frac{m^{3}}{k m o l . s}\right]$, see equation (3.37)

$a_{m+\frac{1}{2}} \quad$ Available width of 'east' $\left(m+\frac{1}{2}\right)$ control surface, $[m]$, page 133

$a_{m-\frac{1}{2}} \quad$ Available width of 'west' $\left(m-\frac{1}{2}\right)$ control surface, $[m]$, page 133 
$A_{\mathrm{NO}_{2}, \text { cake }}$ Passive pre-exponential factor in PM cake layer, $\left[\frac{\mathrm{m}}{\mathrm{s}}\right]$, see equation (3.49)

$A_{N_{2}, \text { wall }}$ Passive pre-exponential factor in substrate wall, $\left[\frac{m}{s}\right]$, see equation (3.52)

$A_{N O} \quad$ Pre-exponential factor of the NO oxidation reaction, $\left[\frac{\mathrm{m}^{3 / 2}}{\mathrm{kmol}^{1 / 2 . s}}\right]$, see equation (3.37)

$A_{p} \quad$ Area of PM per unit mass of PM, $=1 x 10^{5},\left[\frac{\mathrm{m}^{2}}{\mathrm{~kg}}\right]$, see equation (3.49)

$A_{p} \quad$ Area of PM per unit mass of PM, $=1 x 10^{5},\left[\frac{\mathrm{m}^{2}}{\mathrm{~kg}}\right]$, see equation (3.52)

$A_{p} \quad$ Cross-sectional area of the PM cake layer at a given axial $(x)$ interface, $=$ $\frac{a^{*}+a_{c p}}{2} w_{p}\left[m^{2}\right]$, see equation (3.28)

$A_{t h, c a k e} \quad$ Thermal pre-exponential factor in PM cake layer, $\left[\frac{\mathrm{m}}{\mathrm{s}}\right]$, see equation (3.49)

$A_{\text {th,wall }} \quad$ Thermal pre-exponential factor in substrate wall, $\left[\frac{\mathrm{m}}{\mathrm{s}}\right]$, see equation (3.52)

$b \quad$ Wall unit cell diameter, $[m]$, see equation (3.55)

$C$ Stokes-Cunningham slip correction factor $(S C F),=1+$ $K n\left(1.257+(0.4) e^{-1.1 / K n}\right)[$.$] , see equation (3.58)$

$C_{1, \rho_{p w}} \quad$ First constant in wall PM packing density relation to wall PM mass, $\left[\frac{1}{m^{3}}\right]$, see equation (3.12)

$\mathrm{C}_{12} \mathrm{H}_{24} \quad$ n-Dodecene, page 21

$C_{2, \rho_{p w}} \quad$ Second constant in wall PM packing density relation to wall PM mass, $\left[\frac{\mathrm{kg}}{\mathrm{m}^{3}}\right]$, see equation (3.12)

$C_{i} \quad$ Molar concentration of species 'i' $\left[\frac{\mathrm{kmol}}{\mathrm{m}^{3}}\right]$, page 133

$c_{p, m i x} \quad$ Specific heat of the exhaust gas mixture, $\left[\frac{\mathrm{J}}{\mathrm{kg} \cdot \mathrm{K}}\right]$, see equation (3.4)

$c_{p, p} \quad$ Specific heat of the PM cake layer, $\left[\frac{J}{k g . K}\right]$, see equation (3.28)

$c_{p, s} \quad$ Specific heat of substrate wall, $\left[\frac{J}{k g . K}\right]$, see equation (3.28)

$c_{p 1} \quad$ Specific heat (at constant pressure) of the exhaust gas mixture in the inlet channel, $\left[\frac{\mathrm{J}}{\mathrm{kg} \cdot \mathrm{K}}\right]$, see equation (3.25)

$c_{p 2} \quad$ Specific heat (at constant pressure) of the exhaust gas mixture in the outlet channel, $\left[\frac{J}{k g . K}\right]$, see equation (3.26)

$c_{p w} \quad$ Specific heat (at constant pressure) of the exhaust gas mixture in the cake+cat+wall control volume, $\left[\frac{\mathrm{J}}{\mathrm{kg} \cdot \mathrm{K}}\right]$, see equation (3.28)

$\mathrm{CO} \quad$ Carbon monoxide, page 20

$d_{c 0} \quad$ Clean wall collector diameter, $[m]$, see equation (3.55)

$d_{c} \quad$ Instantaneous collector diameter, $[\mathrm{m}]$, see equation (3.58)

$D_{i, j} \quad$ Binary diffusivity of species ' $i$ ' (or species ' $j$ ') in a mixture of ' $i$ ' and ' $j$ ', $\left[\frac{m^{2}}{s}\right]$ , see equation (D.6)

$D_{i} \quad$ Effective diffusivity of chemical species ' $i$ ', $\left[\frac{m^{2}}{s}\right]$, page 133 xxviii 
$D_{K n, i} \quad$ Knudsen diffusivity of chemical species ' $i$ ', $\left[\frac{m^{2}}{s}\right]$, see equation (D.5)

$D_{m o l, i} \quad$ Molecular diffusivity of chemical species ' $i$ ', $\left[\frac{m^{2}}{s}\right]$, see equation (D.5)

$d_{\text {pore }} \quad$ Mean pore size of substrate wall, $[m]$, see equation (3.56)

$D_{p} \quad$ Particle diffusion coefficient, $=\frac{k_{B} T C}{3 \pi \mu d_{p}}\left[\frac{m^{2}}{s}\right]$, see equation (3.58)

$d_{p} \quad$ Primary particle diameter, $=100 \times 10^{-9}[\mathrm{~m}]$, see equation (3.58)

$E a_{C O} \quad$ Activation energy of the $\mathrm{CO}$ oxidation reaction, $\left[\frac{\mathrm{J}}{\mathrm{kmol}}\right]$, see equation (3.37)

$E a_{H C} \quad$ Activation energy of the $\mathrm{HC}$ oxidation reaction, $\left[\frac{\mathrm{J}}{\mathrm{kmol}}\right]$, see equation (3.37)

$E a_{\mathrm{NO}_{2}, \text { cake }}$ Passive activation energy in PM cake layer, $\frac{\mathrm{J}}{\mathrm{kmol}}$, see equation (3.49)

$E a_{\mathrm{NO}_{2} \text {,wall }}$ Passive activation energy in substrate wall, $\frac{\mathrm{J}}{\mathrm{kmol}}$, see equation (3.52)

$E a_{N O} \quad$ Activation energy of the NO oxidation reaction, $\left[\frac{\mathrm{J}}{\mathrm{kmol}}\right]$, see equation (3.37)

$E a_{\text {th,cake }} \quad$ Thermal activation energy in PM cake layer, $\frac{\mathrm{J}}{\mathrm{kmol}}$, see equation (3.49)

$E a_{t h, w a l l} \quad$ Thermal activation energy in substrate wall, $\frac{J}{k m o l}$, see equation (3.52)

$F \quad$ Friction factor in the inlet channel and outlet channel of the particulate filter, [.] , see equation (B.13)

$g \quad$ Acceleration due to gravity, $\left[\frac{m}{s^{2}}\right]$, see equation (C.1)

$h_{1} \quad$ Heat transfer coefficient for convective heat transfer between inlet channel and substrate wall, $\left[\frac{W}{m^{2} K}\right]$, see equation (3.11)

$h_{2} \quad$ Heat transfer coefficient for convective heat transfer between outlet channel and substrate wall, $\left[\frac{W}{m^{2} K}\right]$, see equation (3.11)

$h_{a m b} \quad$ Convection heat transfer coefficient, $\left[\frac{W}{m^{2} . K}\right]$, see equation (3.28)

$H_{i} \quad$ Heat of reaction for a chemical reaction occurring in the PM cake layer, $\left[\frac{J}{k m o l . K}\right]$, see equation (3.28)

$H_{j} \quad$ Heat of reaction for a chemical reaction occurring in the catalyst washcoat, $\left[\frac{J}{k m o l . K}\right]$, see equation (3.28)

$H_{k} \quad$ Heat of reaction for a chemical reaction occurring in the substrate wall, $\left[\frac{J}{k m o l . K}\right]$, see equation (3.28)

$H C$ 's Hydrocarbons, page 20

$K \quad$ Kuwabara's hydrodynamic factor, [.] , see equation (3.58)

$k_{B} \quad$ Boltzmann constant, $=1.38 \times 10^{-23}\left[\frac{\mathrm{J}}{K}\right]$, see equation (3.58)

$k_{i} \quad$ Thermal conductivity of individual chemical species, $\left[\frac{W}{m K}\right]$, see equation (3.10)

$k_{\text {mix }} \quad$ Thermal conductivity of exhaust gas mixture, $\left[\frac{W}{m K}\right]$, see equation (3.10)

$k_{s} \quad$ Equivalent permeability of the substrate wall of the particulate filter, $\left[\mathrm{m}^{2}\right]$, see equation (B.14)

$K n \quad$ Local Knudsen number, $=\frac{2 \lambda}{d_{\text {pore }}}[K]$, see equation (3.58)

$M \quad$ Mass flux at the inlet of the particulate filter, $=\frac{\dot{m}}{n_{\text {cells }}\left(a^{*}\right)^{2}}\left[\frac{\mathrm{kg}}{\mathrm{m}^{2} s}\right]$, see equation (B.12) 
$m+1 \quad$ 'Succeeding' node of node ' $m$ ' (refer Figure D.3)

$m+\frac{1}{2} \quad$ 'East' control surface of node ' $m$ ' (refer Figure D.3)

$m-1 \quad$ 'Previous' node of node ' $m$ ' (refer Figure D.3)

$m-\frac{1}{2} \quad$ 'West' control surface of node ' $m$ ' (refer Figure D.3)

$m_{\text {cake }}$

Mass of PM in the PM cake layer at any given instant, $[k g]$, see equation (3.48)

$m_{\text {wall }}$

$m_{\text {wall }}$

Mass of PM in the substrate wall at any given instant, $[k g]$, see equation (3.48)

equation (3.12)

$M W_{\text {exh }}$ Equivalent molecular weight of exhaust gas mixture, $\left[\frac{\mathrm{kg}}{\mathrm{kmol}}\right]$, see equation (3.58)

$M W_{i} \quad$ Molecular weight of species 'i' $\left[\frac{\mathrm{kg}}{\mathrm{kmol}}\right]$, page 133

$n_{\text {cells }} \quad$ Number of inlet cells in the CPF, [.], see equation (B.9)

$n_{r k} \quad$ Number of chemical reactions involving gaseous chemical species, [.], see equation (3.5)

$N_{R} \quad$ Particle interception parameter, $=\frac{d_{p}}{d_{c}}[$.$] , see equation (3.59)$

$n_{s p} \quad$ Number of gaseous chemical species, [.] , see equation (3.5)

NO Nitric oxide, page 20

$\mathrm{NO}_{2} \quad$ Nitrogen dioxide, page 20

$N u \quad$ (Limiting value of) Nusselt number for square channels, $=2.975$ [.] , see equation (3.11)

$P \quad$ Absolute pressure of exhaust gas, $[\mathrm{Pa}]$, see equation (3.58)

$P_{1} \quad$ Absolute pressure of the exhaust gas mixture in the inlet channel of the particulate filter, $[\mathrm{Pa}]$, see equation (B.13)

$P_{2} \quad$ Absolute pressure of the exhaust gas mixture in the outlet channel of the particulate filter, $[\mathrm{Pa}]$, see equation (B.13)

$P_{\text {exit }} \quad$ Measured CPF exit pressure, $[P a]$, page 53

$P_{w} \quad$ Absolute pressure of exhaust gas mixture in the cake+cat+wall control volume, $=\frac{P_{1}+P_{2}}{2}[P a]$, see equation (3.1)

$P e \quad$ Peclet number, $=\frac{v_{i} d_{c}}{D_{p}}[$.$] , see equation (3.58)$

$R_{C O} \quad$ Rate of $\mathrm{CO}$ oxidation reaction, $\left[\frac{\mathrm{kmol}}{\mathrm{m}^{3} . \mathrm{s}}\right]$, see equation (3.37)

$R_{H C} \quad$ Rate of $\mathrm{HC}$ oxidation reaction, $\left[\frac{\mathrm{kmol}}{\mathrm{m}^{3} \cdot \mathrm{s}}\right]$, see equation (3.37)

$R_{i} \quad$ Rate of a chemical reaction occurring in the PM cake layer, $\left[\frac{\mathrm{kmol}}{\mathrm{m}^{3} . \mathrm{s}}\right]$, see equation (3.28)

$R_{j} \quad$ Rate of a chemical reaction occurring in the catalyst washcoat, $\left[\frac{\mathrm{kmol}}{\mathrm{m}^{3} \cdot \mathrm{s}}\right]$, see equation (3.28)

$R_{j} \quad$ Rate of reaction $j,\left[\frac{\mathrm{kmol}}{\mathrm{m}^{3} \mathrm{~s}}\right]$, see equation (3.35)

$R_{j} \quad$ Rate of reaction ' $j$ ', $\left[\frac{\mathrm{kmol}}{\mathrm{m}^{3} \mathrm{~s}}\right]$, page 133 
$R_{k} \quad$ Rate of a chemical reaction occurring in the substrate wall, $\left[\frac{\mathrm{kmol}}{\mathrm{m}^{3} \cdot \mathrm{s}}\right]$, see equation (3.28)

$R_{\mathrm{NO}_{2}, \text { cake }}$ Reaction rate of passive PM oxidation reaction in the PM cake layer, $\left[\frac{\mathrm{kmol}}{\mathrm{m}^{3} . \mathrm{s}}\right]$, see equation (3.48)

$R_{N O} \quad$ Rate of NO oxidation reaction, $\left[\frac{\mathrm{kmol}}{\mathrm{m}^{3} \cdot \mathrm{s}}\right]$, see equation (3.37)

$R_{\text {th,cake }} \quad$ Reaction rate of thermal PM oxidation reaction in the PM cake layer, $\left[\frac{\mathrm{kmol}}{\mathrm{m}^{3} \cdot \mathrm{s}}\right]$, see equation (3.48)

$S_{p} \quad$ Specific area of PM, $=\rho_{p} A_{p},\left[\frac{m^{2}}{m^{3}}\right]$, see equation (3.49)

$S_{p} \quad$ Specific area of $\mathrm{PM},=\rho_{p} A_{p},\left[\frac{m^{2}}{m^{3}}\right]$, see equation (3.52)

$T \quad$ Absolute temperature, $[K]$, see equation (3.58)

$T_{1} \quad$ Absolute temperature of the exhaust gas mixture in the inlet channel, $[K]$, see equation (3.25)

$T_{2} \quad$ Absolute temperature of the exhaust gas mixture in the outlet channel, $[K]$, see equation (3.26)

$T_{a m b} \quad$ Absolute ambient temperature, $[K]$, see equation (3.28)

$T_{w} \quad$ Absolute temperature of the cake+cat+wall control volume, $[K]$, see equation (3.28)

$V \quad$ Volume of control volume, $\left[\mathrm{m}^{3}\right]$

$v \quad$ Velocity of the fluid in the control volume, $\left[\frac{m}{s}\right]$, see equation (C.1)

$v_{1} \quad$ Velocity of exhaust gas mixture in the inlet channel control volume, $\left[\frac{\mathrm{m}}{\mathrm{s}}\right]$, see equation (B.1)

$v_{2} \quad$ Velocity of exhaust gas mixture in the outlet channel control volume, $\left[\frac{m}{s}\right]$, see equation (B.1)

$v_{i} \quad$ Interstitial velocity, $=\frac{v_{w}}{\epsilon}\left[\frac{m}{s}\right]$, see equation (3.58)

$v_{w} \quad$ Velocity of exhaust gas mixture at the exit (boundary $B_{2}$ ) of the cake+cat+wall control volume, $\left[\frac{\mathrm{m}}{\mathrm{s}}\right]$, see equation (B.1)

$v_{w}^{*} \quad$ Velocity of the exhaust gas mixture at the entrance of the cake+cat+wall control volume (Boundary $\left.B_{1}\right),\left[\frac{m}{s}\right]$, page 121

$w_{\text {cat }} \quad$ Thickness of catalyst washcoat on top of the substrate wall, $[\mathrm{m}]$, page 31

$w_{p} \quad$ Thickness of PM cake layer, $[m]$, see equation (3.65)

$w_{p} \quad$ Thickness of the PM cake layer formed on the substrate wall of the particulate filter, $[m]$, see equation (B.14)

$w_{s} \quad$ Thickness of the substrate wall of the particulate filter, $[m]$, see equation (B.14)

$x \quad$ Independent variable which denotes axial location [.] , page 121

$x_{C O} \quad$ Temperature order of dependence of the $\mathrm{CO}$ oxidation reaction, [.] , see equation (3.37) 
$x_{H C} \quad$ Temperature order of dependence of the $\mathrm{HC}$ oxidation reaction, [.] , see equation (3.37)

$x_{\mathrm{NO}_{2}, \text { cake }}$ Passive temperature order of dependence in PM cake layer, [.] , see equation (3.49)

$x_{\mathrm{NO}_{2} \text {,wall }}$ Passive temperature order of dependence in substrate wall, [.] , see equation (3.52)

$x_{N O} \quad$ Temperature order of dependence of the NO oxidation reaction, [.], see equation (3.37)

$x_{\text {th,cake }}$ Thermal temperature order of dependence in PM cake layer, [.] , see equation (3.49)

$x_{t h, \text { wall }}$ Thermal temperature order of dependence in substrate wall, [.] , see equation (3.52)

$Y_{1, i} \quad$ Mole fraction of chemical species ' $i$ ' in the inlet channel control volume, $\left[\frac{k m o l_{i}}{k^{\prime} o_{e x h}}\right]$, page 26

$Y_{2, i} \quad$ Mole fraction of chemical species ' $i$ ' in the outlet channel control volume, $\left[\frac{k_{m o l}}{k_{m o l} l_{e x h}}\right]$, page 26

$Y_{i} \quad$ Mole fraction of chemical species ' $i$ ' in the cake+cat+wall control volume, $\left[\frac{k m o l_{i}}{k_{m o l} l_{e x h}}\right]$, page 26

$z \quad$ Height of the control volume from a reference point, $[m]$, see equation (C.1)

API Americal Petroleum Institute, page 58

AR Active Regeneration

cake+cat+wall PM cake layer, catalyst washcoat and substrate wall control volume , page 22

CPF Catalyzed (Diesel) Particulate Filter

DOC Diesel Oxidation Catalyst

DPF Diesel Particulate Filter

ICP-AES Inductively Coupled Plasma Atomic Emission Spectrometry, page 58

IR Infra-Red, page 58

OBD On-Board Diagnostics

PO Passive Oxidation

SCR Selective Catalytic Reduction catalyst 


\title{
Development of a 1-D Catalyzed Diesel Particulate Filter Model for Simulation of the Performance and the Oxidation of Particulate Matter and Nitrogen Oxides Using Passive Oxidation and Active Regeneration Engine Experimental Data
}

\author{
Kiran C. Premchand \\ Michigan Technological University, 2013 \\ Advisor: Dr. John H. Johnson
}

\begin{abstract}
Back-pressure on a diesel engine equipped with an aftertreatment system is a function of the pressure drop across the individual components of the aftertreatment system, typically, a diesel oxidation catalyst (DOC), catalyzed particulate filter (CPF) and selective catalytic reduction (SCR) catalyst. Pressure drop across the CPF is a function of the mass flow rate and the temperature of the exhaust flowing through it as well as the mass of particulate matter (PM) retained in the substrate wall and the cake layer that forms on the substrate wall. Therefore, in order to control the back-pressure on the engine at low levels and to minimize the fuel consumption, it is important to control the PM mass retained in the CPF. Chemical reactions involving the oxidation of PM under passive oxidation and active regeneration conditions can be utilized with computer numerical models in the engine control unit (ECU) to control the pressure drop across the CPF. Hence, understanding and predicting the filtration and oxidation of PM in the CPF and the effect of these processes on the pressure drop across the $\mathrm{CPF}$ are necessary for developing control strategies for the aftertreatment system to reduce back-pressure on the engine and in turn fuel consumption particularly from active regeneration. Numerical modeling of CPF's has been proven to reduce development time and the cost of aftertreatment systems used in production as well as to facilitate understanding of the internal processes occurring during different operating conditions that the particulate filter is subjected to. A numerical model of the CPF was developed in this research work which was calibrated to data from passive oxidation and active regeneration experiments in order to determine the kinetic parameters for oxidation of PM and nitrogen oxides along with the model filtration parameters. The research results include the comparison between the model and the experimental data for pressure drop, PM mass retained, filtration efficiencies, CPF outlet gas temperatures and species $\left(\mathrm{NO}_{2}\right)$ concentrations out of the CPF. Comparisons of PM oxidation reaction rates obtained from the model calibration to the data from the experiments for ULSD, 10 and $20 \%$ biodiesel-blended fuels are presented.
\end{abstract}





\section{INTRODUCTION ${ }^{1}$}

Increasingly stringent PM emission standards have prompted researchers in the area of engine exhaust emissions reduction to explore and develop technology to reduce PM emission levels from diesel engines below EPA standards. The diesel particulate filter (DPF) [1] is an important component of current diesel exhaust aftertreatment systems that enable diesel engines to operate with tailpipe PM emissions that meet these standards. One of the more common configurations for a diesel exhaust aftertreatment system consists of a diesel oxidation catalyst (DOC), a catalyzed particulate filter (CPF) and a selective catalytic reduction (SCR) catalyst [2]. The DOC [3] is a flow-through device composed of numerous square channels separated by the substrate walls which are coated with a catalyst washcoat consisting of noble metals such as platinum $(\mathrm{Pt})$ and/or palladium $(\mathrm{Pd})$. The purpose of the catalyst in the DOC is to oxidize gaseous species such as hydrocarbons $(H C)$, carbon monoxide $(\mathrm{CO})$ and nitric oxide $(\mathrm{NO})$ to nitrogen dioxide $\left(\mathrm{NO}_{2}\right)$ which is an oxidant of solid carbonaceous particulate matter. The primary function of the CPF is to filter and oxidize diesel particulate matter. The CPF is typically a wall-flow device consisting of square channels plugged at alternate ends in adjacent channels such that exhaust gas mixture passes through the walls of the porous substrate. Particulate matter (PM) is physically filtered by the $\mathrm{CPF}$ and oxidized to form carbon monoxide $(\mathrm{CO})$ and carbon dioxide $\left(\mathrm{CO}_{2}\right)$. Also, the carbon monoxide formed in the $\mathrm{CPF}$ is further oxidized with oxygen to carbon dioxide. In addition, the carbon monoxide and hydrocarbons out of the DOC and into the CPF are also oxidized to form water $\left(\mathrm{H}_{2} \mathrm{O}\right)$ and $\mathrm{CO}_{2}$. Particulate matter is deposited in the pores of the substrate wall at the onset of the filtration process (known as "deep-bed filtration") and eventually, on the inlet channel surfaces of the substrate wall as a separate PM cake layer, which then forms a highly efficient filter for the incoming PM, resulting in near $97 \%$ filtration efficiencies of the $\mathrm{CPF}$ [4]. This part of the operating range of the particulate filter is commonly referred to as cake filtration regime, characterized by a linear increase in pressure drop across the filter with respect to loading time for constant engine operating conditions.

Back pressure on the diesel engine is a function of the pressure drop across the DOC,

\footnotetext{
${ }^{1}$ Parts of the material contained in this chapter have been published, or are currently under consideration for publication by SAE International.
} 
CPF and SCR. The pressure drop across the DOC and the SCR are functions of mass flow rate and temperature of the exhaust gas while the pressure drop across the CPF is a function not only of exhaust gas mass flow rate and temperature but also of the amount of carbonaceous particulate matter (PM) retained in the CPF. Hence, to control the pressure drop across the CPF, it is important to control the PM mass retained in the CPF. Chemical reactions involving the oxidation of PM under passive oxidation and active regeneration conditions can be used with models in the engine control unit (ECU) to control the pressure drop across the CPF. Understanding and predicting the oxidation of PM and its effect on pressure drop across the $\mathrm{CPF}$ is necessary for developing aftertreatment control strategies to reduce fuel consumption.

The performance of the CPF under different engine operating conditions can be studied by an experimentally validated numerical model. Numerical modeling has been used to reduce the time and cost associated with the development of aftertreatment systems for an engine in a specific application. The motivation behind this research work has been the need to develop a numerical model for a CPF which is capable of simulating all operating conditions that a $\mathrm{CPF}$ is subjected to and is able to predict the major performance characteristics of the $\mathrm{CPF}$ during these operating conditions. Also, this numerical model needs to be calibrated to engine experimental data which would then give confidence that the model can be used to predict CPF performance.

\subsection{Research Objectives}

The specific objectives of this research work were to:

1. Develop a 1-D numerical model of a CPF capable of predicting flow and filtration variables, filtration and oxidation of PM and oxidation of gaseous species and back-diffusion of $\mathrm{NO}_{2}$ involved during loading, passive oxidation, active regeneration and post-loading, including specifically:

- a literature review of the papers that have been used to develop the model and to explain the research results,

- a catalyst sub-model that predicts gaseous species concentrations as the exhaust gas mixture flows through the PM cake layer, catalyst and substrate wall of the $\mathrm{CPF}$ and undergoes reactions with other gaseous species and/or solid particulate matter, including a framework for diffusion of gaseous species in addition to convection, and oxidation of PM in the PM cake layer and substrate wall through oxidation with $\mathrm{O}_{2}$ and $\mathrm{NO}_{2}$,

- a temperature sub-model that predicts the temperature of the exhaust gas as it 
passes through the inlet channels, the substrate wall and outlet channels of the particulate filter,

- a pressure sub-model that predicts the absolute pressure at each axial location in the inlet and outlet channels of the single channel representation of the particulate filter as a function of time during a particular experiment,

- a velocity sub-model that predicts the gaseous velocity of the gas mixture as it passes through the inlet channels, substrate wall and outlet channels, and

- a filtration model that predicts the mass of particulate matter retained in the PM cake layer and substrate wall.

2. Develop a calibration procedure to determine the parameters related to filtration, catalyst reaction kinetics and PM kinetics that need to be specified for the CPF model in order to simulate the experimental data from passive oxidation (PO) and active regeneration $(\mathrm{AR})$ engine experiments with a full-scale $\mathrm{CPF}$,

3. Quantify the differences between the model results and experimental measurements,

4. Arrive at optimized values of kinetic parameters for PM oxidation with $\mathrm{NO}_{2}$ and $\mathrm{O}_{2}$ and kinetic parameters for the oxidation reaction involving oxides of nitrogen ( $\mathrm{NO}$ and $\mathrm{NO}_{2}$ ), carbon monoxide $(\mathrm{CO})$ and hydrocarbons $(\mathrm{HC})$ and also filtration parameters needed to simulate the total pressure drop and PM retained in the cake layer and substrate wall,

5. Determine the effect of fuel type (ULSD, B10, B20) on rates of $\mathrm{NO}_{2}$-assisted and thermal $\left(\mathrm{O}_{2}\right)$ PM oxidation reactions and parametrically simulate the reaction rates as a function of temperature and $\mathrm{NO}_{2}$ concentration,

6. Determine the effect of PM cake layer and substrate wall PM oxidation rates on overall pressure drop $(\Delta P)$ across the $\mathrm{CPF}$,

7. Determine the effect of back-diffusion of $\mathrm{NO}_{2}$ on oxidation of PM with $\mathrm{NO}_{2}$, and

8. Determine the effect of the experiments (both PO and AR) on PM cake layer thickness calculated by the model.

\subsection{Overview of Thesis}

This dissertation describes research effort directed towards development and application of the MTU CPF 1-D model version 4.0 to data from passive oxidation and active regeneration experiments, and is organized into six chapters and twelve appendices

that are cross-referenced from the various chapters. This chapter (Chapter 1) gives an 
introduction to this research work and objectives and explains the general outline of the thesis. Chapter 2 is a description of a literature review conducted as part of this research work to study existing 1-D CPF models and literature relevant that were needed to develop the model. Chapter 3 gives a description of the model developed. Chapter 4 describes the experimental data collected using a Cummins ISL diesel engine and the development of a procedure to calibrate the CPF model, and the process of calibration. Chapter 5 gives a detailed description of the results obtained from simulations using the CPF model and extensive comparisons between the experimental data and model results. The chapter also shows and explains the PM reaction rate data and the kinetic parameters for ULSD, B10 and B20 that were determined from the calibration of the model to the experimental data. Chapter 6 summarizes this research work and presents the conclusions drawn from model results about the performance of the CPF during passive oxidation and active regeneration experiments. 


\section{BACKGROUND AND LITERATURE REVIEW ${ }^{1}$}

One of the objectives of the research work was the development of a CPF model. Hence, a thorough study of the published literature on the topic was needed. The overall objective was to study existing numerical CPF models that take into consideration the filtration and oxidation of PM and oxidation of gaseous species such as hydrocarbons, carbon monoxide and nitric oxide. The literature that was reviewed were on the topics of modeling filtration of PM by the substrate wall and the PM cake layer, modeling properties of particulate matter collected in a CPF, oxidation of PM via $\mathrm{NO}_{2}$-assisted and thermal $\left(\mathrm{O}_{2}\right)$ reaction mechanisms, gaseous oxidation reactions involving hydrocarbons, $C O, N O$ and $\mathrm{NO}_{2}$ including modeling of back-diffusion of $\mathrm{NO}_{2}$. The following paragraphs are brief descriptions of published papers that describe numerical models developed for studying particulate filters.

\subsection{1-D CPF Models}

Among the first published works about numerical modeling of catalytic converters were those from General Motors Research Lab [5,6]. The focus of these studies was the transient behavior of automobile catalytic converters using a mathematical model considering the simultaneous processes of heat transfer, mass transfer and chemical reaction where the response of the catalyst monolith to s step increase in the feed-stream temperature was examined in order to understand the thermal characteristics of a catalyst to rapidly changing inlet temperature in real-life situations. It is also important to note here that the catalyst used in this study was flow-through (not wall-flow, such as in DPF).

The earliest published works about diesel particulate filter modeling were also from General Motors Research Labs [7-9] where a mathematical model was developed to study the thermal regeneration of a wall-flow diesel particulate filter where conservation equations regarding the mass, momentum and energy of exhaust gas as it flows through a

\footnotetext{
${ }^{1}$ Parts of the material contained in this chapter have been published, or are currently under consideration for publication by SAE International.
} 
wall-flow monolith as well as energy of the wall were used to predict the wall and outlet gas temperatures during a regeneration caused by an increase in inlet feed-gas temperature. Here, an elementary pressure drop model was used which considered the total pressure drop as that occurring across the PM cake layer and substrate wall. This set of works was significant in that the basic framework of the 1-D model of a diesel particulate filter has remained largely unchanged even $t$ the present day.

Another work about the modeling of the regeneration behavior of diesel particulate traps appeared in SAE [10] in 1983. This study talked, among other findings, about the influence of particle load variation on the axial flow distribution. The results compared were the model-predicted and measured values of filter temperature during a thermal regeneration event where the inlet temperature was raised from $400{ }^{\circ} \mathrm{C}$ to $650{ }^{\circ} \mathrm{C}$ in about 2 minutes. Maximum temperatures of $790{ }^{\circ} \mathrm{C}$ were calculated and $800{ }^{\circ} \mathrm{C}$ were measured. This work also stated that the model predictions were further validated by the practical observation that the maximum thermal stress occurred primarily at the rear of the filter.

The first significant research work towards development of filtration of particulate matter by the substrate wall of a diesel particulate filter was published in 1989 [11]. This work focused on the development of a diesel particulate filter model which had the same framework as in reference [8], but incorporated a filtration model from a study conducted on collection of aerosol particles in a packed bed [12]. This work incorporated flow and filtration models to predict the pressure drop across the clean filter wall.

Subsequent works in the field of numerical modeling of DPF's have largely followed the framework set by Bissett [8] and Konstandopoulos et al [11]. Konstandopoulos et al extended the clean pressure drop model described in reference [11] to an arbitrary filter loading and revised the pressure drop model in reference [13]. Other research works also studied the pressure drop model $[14,15]$ and published validated models showing comparisons of model and experimental pressure drops for both clean and PM-loaded filters. Another notable research work was published by the same research group in 2001 [16] which described the development of a multi-channel axi-symmetric numerical model to capture spatial non-uniformities involved in the regeneration of diesel particulate filters. For this framework, the governing equations for mass, axial momentum and energy balance were formulated involving 3 length scales - filter wall, single channel and entire filter. This model was validated by comparing the model to measured temperature from thermocouple readings during active regeneration of a cordierite DPF.

A research work published in 2001 [17] discussed the development of a transient quasi 1-dimensional model to study the effect of substrate material and geometric properties on regeneration characteristics of a $\mathrm{SiC}$ particulate filter (NGK). This study focused on predicting the temperature gradients during a forced regeneration using a constant temperature feed-gas $(600 \circ \mathrm{C})$ using the transient model and comparing them 
to experimental data as re-produced in Figure 2.1. Also demonstrated were the effects of filter physical properties such as thermal conductivity and heat capacity on maximum temperature during regeneration from the model-predicted results.

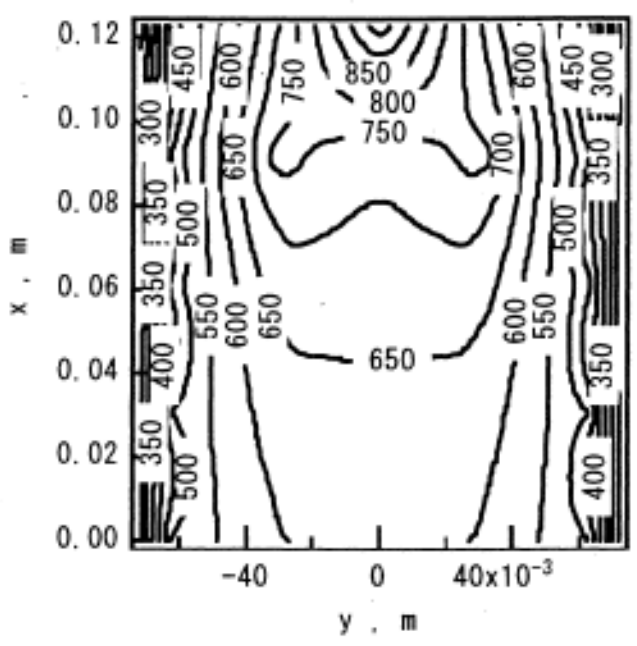

[a] Experimentally measured result for Cordierite Cell density: $200 \mathrm{cpsi}$, Wall thickness: 14 mil

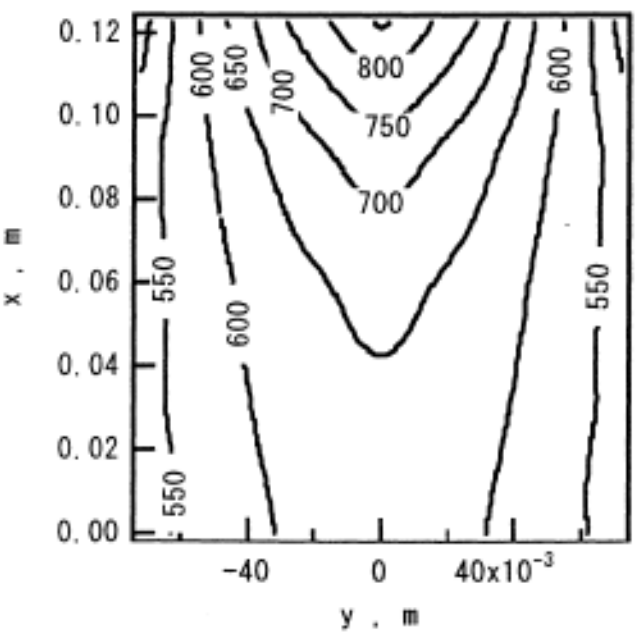

[c] Experimentally measured result for SiC Cell density: $300 \mathrm{cpsi}$, Wall thickness:12 mil

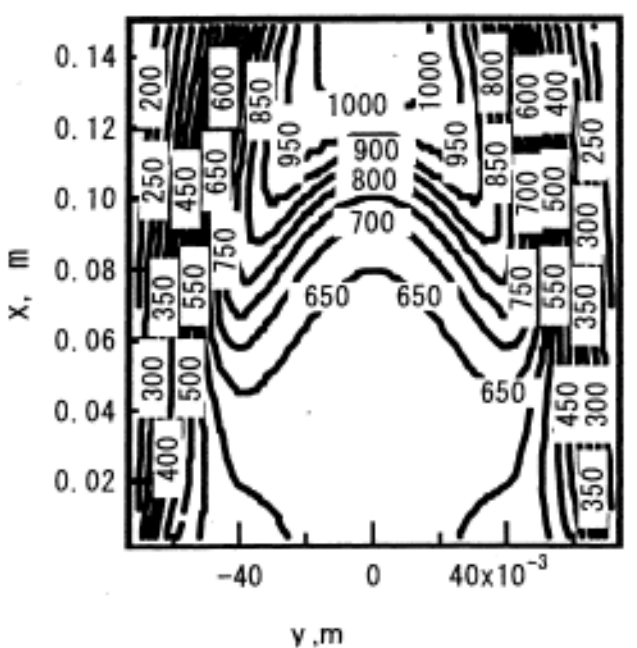

[b] Model calculation result for Cordierite Cell density:200 cpsi, Wall thickness: 14mil

Figure 2.1: Comparison of thermal contours during a forced regeneration event from experiments and model [17] - Reprinted with permission from SAE Paper No. 2001-01-0912 (C)2001 SAE International. 
Another research work that expanded further on a multi-channel model was published in 2003 [18]. The model developed in reference [16] was used in this work to study the effect of filter can heat losses, local hot spots and inlet non-uniformities (for example, the mass flux and temperature at the inlet side of various inlet channels as shown from a CFD simulation in reference [18]) on PM mass fraction and wall temperature evolution. This was the first published work on the use of a 1-D model which also showed good agreement with results from a 3-D model [16].

Authors from MTU have published works about 2-dimensional models for diesel particulate filters [19,20], which were published in 2 parts: the first part [19] described the flow and filtration characteristics including wall-flow velocity and pressure drop while the second part [20] focused on the heat transfer, reaction kinetics and regeneration characteristics of the DPF. The authors concluded that the model developed was applicable for any operating condition, although the validity of the model was proven using steady-state loading and regeneration tests.

Reference [21] is a comprehensive review work that explains the development of a 1-D model of a diesel particulate filter where the concept of area conservation due to build-up of particulate matter was explained and implemented in all governing equations developed for the model, along with comparisons from other research works which assumed constant cross-sectional area of the inlet channel in spite of accumulation of PM in the PM cake layer. This proved useful in the MTU 1-D model during the derivation of governing equations (Appendices A, B, C and D).

Other research works of interest about CPF models are the publications of 3D modeling of diesel particulate filters by Koltsakis et al. in 2005 [22,23], 3-D models [24,25] another 2D catalyzed filter regeneration model by Frey et al in 2007 [26], the use of a 3D model (Axitrap - commercial DPF simulation software by Exothermia[27]) for the optimization of catalyst zoning in diesel particulate filters in 2008 by Koltsakis et al. [28] and also presented at the 6th International CTI exhaust forum in Nurtingen, Germany in 2008 [29]. Finite volume-based approaches have also been successfully implemented to study the flow processes inside a diesel particulate filter, such as those published in [30] and [31].

More recently, research efforts focused on a filtration model that is able to predict the evolution of filtration efficiency of particulate filters including the transition from wall filtration to cake filtration was published in references [32,33] which assisted the development of the logic of the filtration model developed at MTU. The following sections describe the research works that were relevant to specific improvements made in the MTU CPF high-fidelity model.

A recent comprehensive review on the current state of CPF modeling can be found in reference [34]. The review covers early history of the modeling of diesel particulate 
filters and revisits all governing equations related to flow and filtration in particulate filters. Results from the authors' models are compared to other works and presented. Also, other topics such as thermal stresses, ash loading, 3-dimensional modeling of diesel particulate filters, asymmetric channels and non-homogeneous wall structure are considered. The authors conclude that the computational tools used are not only design and optimization aids but also an integral part of the development process of exhaust aftertreatment systems.

The following sections describe the literature relevant to important aspects of CPF modeling carried out as part of this research work.

\subsection{Filtration and Deposition of PM}

Filtration of particulate mater is the primary function of a particulate filter. Figure 2.2 shows a cutaway representation of the flow of exhaust gas through the porous wall of the substrate of a typical diesel particulate filter. A DPF is made out of several possible materials, out of which a ceramic called Cordierite is the most popular option. Cordierite is a magnesium aluminum cyclosilicate $\left(2 \mathrm{MgO}^{2} 2 \mathrm{Al}_{2} \mathrm{O}_{3} .5 \mathrm{SiO}_{2}\right)$, named after a French geologist Louis Cordier, and was discovered in 1813. The typical particulate filter is composed of numerous square channels with alternate ends plugged and the walls are porous (with clean porosity of about 50\%) which forces the exhaust gas to pass through the walls of the substrate as shown in Figure 2.2. Majority of particulate matter is captured in and on the porous walls of the substrate, thereby physically separating it from the exhaust gas. The clean gas mixture then exits through the outlet end of the particulate filter.

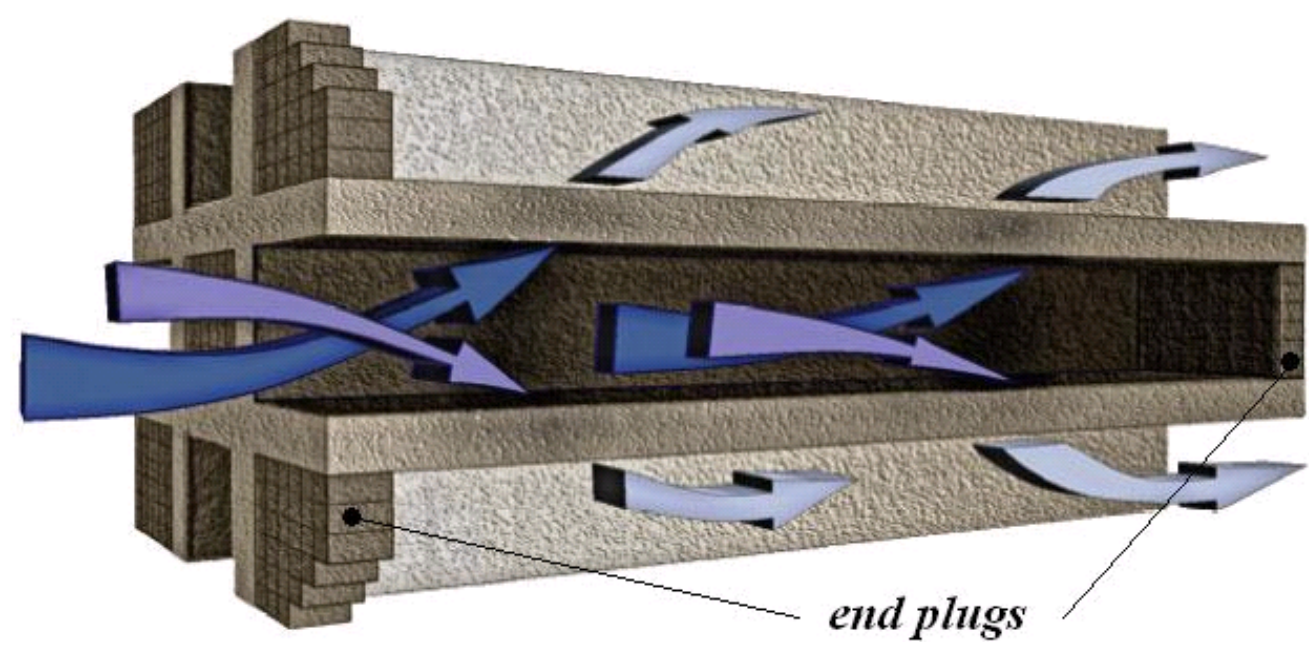

Figure 2.2: Schematic representation of flow of exhaust gas mixture in a wall-flow type particulate filter - adapted from reference [35] 
As PM is carried into the pores of the substrate wall, deposition of PM may take place via the following mechanisms:

- Diffusion,

- Interception,

- Inertial impaction,

- Gravitational deposition,

- Electrostatic deposition, and

- Thermophoresis,

The filtration efficiencies of these different mechanisms have been shown in reference [36] as functions of particle sizes for a specific filtration medium and flow conditions as shown in Figure 2.3. The filtration of particles by the diffusion mechanism decreases with increasing particle size since diffusion of smaller particle diameters will occur faster. The interception mechanism captures particles through direct interception of the particle by the spherical collector and hence the efficiency of this mechanism increases as the particle size increases. Also, Inertial and gravitational collection efficiencies generally increase with particle size since both are directly dependent on the mass of the particle.

The first research work that calculated the efficiency of particle capture via these different mechanisms [12] uses the Kuwabara model to predict the collection of aerosol particles from flow through a packed bed. It takes into account the deposition through two of the afore-mentioned mechanisms: diffusion and interception. The filtration efficiency through diffusion and interception were calculated as functions of flow and geometric properties as well as the size of the particles captured. The equivalent filtration efficiency of the spherical collector was calculated as the resultant of the two individual mechanisms assumed to be acting independent of each other.

The first application of filtration theory that was published in reference [12] specifically to diesel particulate filters was in a research paper by Konstandopoulos and Johnson [11], where the filtration efficiency of the substrate wall of the particulate filter was modeled to simulate capture of diesel particulate matter occurring inside a diesel particulate filter. The capture mechanisms considered were diffusion and interception. The substrate wall was assumed to consist of a number of unit collectors capable of capturing incoming particulate matter at equivalent filtration efficiencies depending on flow conditions, geometric properties of the substrate wall and diameter of incoming particle. This work also asserts that for typical flow velocities and size of diesel PM, the inertial impaction has 


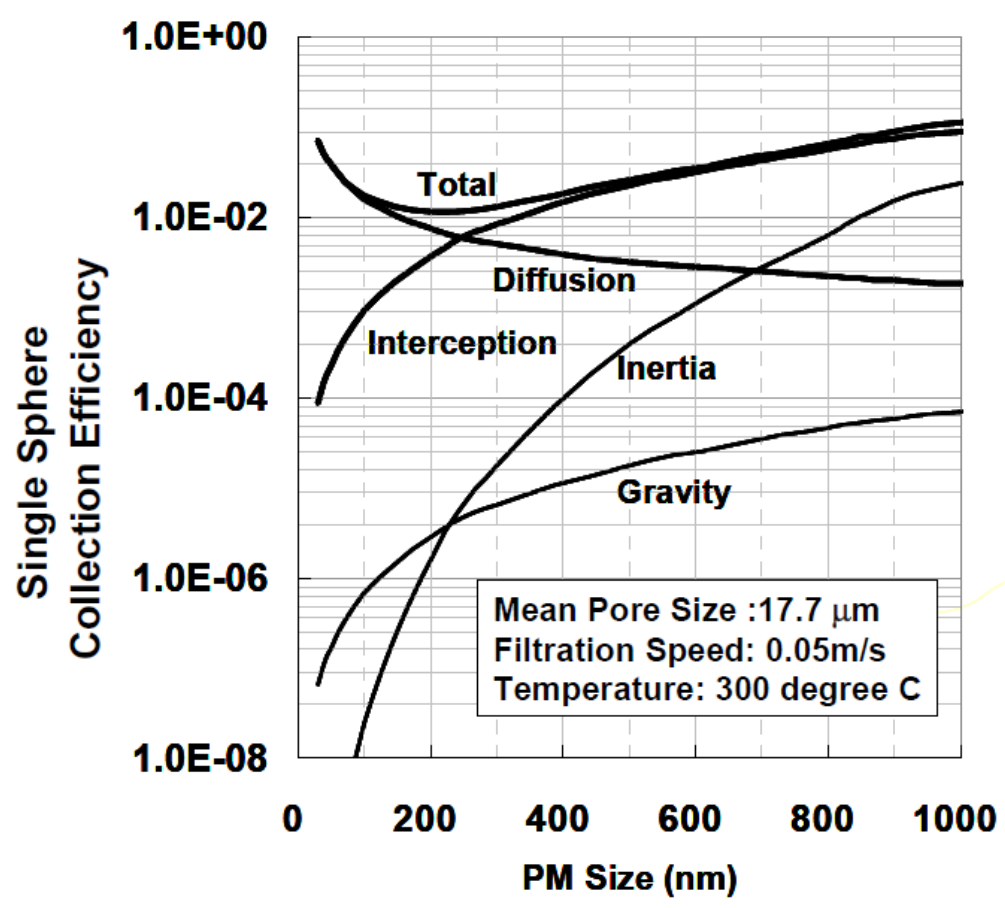

Figure 2.3: Unit collector filtration efficiency [36] - Reprinted with permission from SAE Paper No. 2007-01-0921 (c)2007 SAE International.

a low impact on total filtration efficiency and hence can be ignored. The work presented in this thesis follows the same approach and the equations for calculating filtration-related variables in the CPF high-fidelity model are as given in 3.4.7.

Filtration of PM by the substrate wall leads to the formation of a PM cake layer. This PM cake layer then forms a filter of high (>90\%) filtration efficiency itself. One mechanism for modeling this transition was discussed in a recent research work by Corning [33] which was an extension of existing research work by the same research group [32]. These works used the concept of a transition permeability of the filter wall to use as a deciding factor in the modeling of the transition from wall (deep-bed) filtration to cake filtration. Also presented in reference [32] were parametric studies of the pressure drop and filtration efficiency of a clean filter as functions of geometric properties of the filter substrate such as porosity and mean pore size as well as measured time-dependent filtration efficiencies of filters of different geometries as shown re-produced in Figure 2.4. This proved the validity of the approach that was being followed by the author for PM cake filtration in order to predict filtration efficiency evolution which is shown later as being similar in trend.

The filtration of PM by the PM cake layer itself is the primary reason for the high $(>95 \%)$ filtration efficiencies that are observed during most operating conditions that the CPF undergoes [33]. The PM cake layer formed on the substrate wall is the result of 


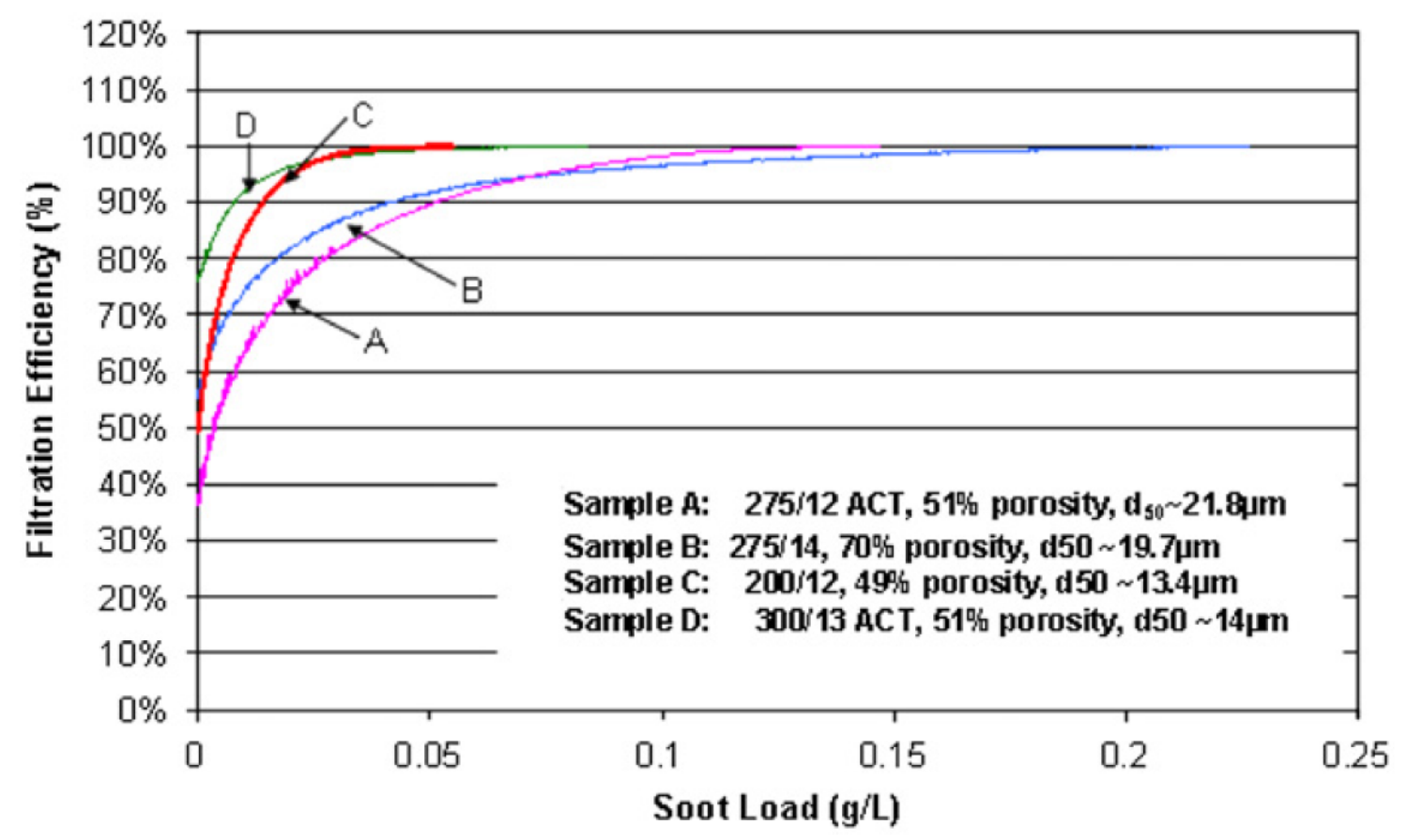

Figure 2.4: Measured filtration efficiency evolution for 4 different filters having different filter microstructure and geometry properties. Re-produced from reference [32]

PM being convected into the pores in the substrate wall by the exhaust gas. The velocity of flow of this exhaust gas mixture is not uniform axially [19]. The profile of wall-flow velocity has been shown to be higher at the inlet end and outlet end than in the middle of the length of the substrate from both an analytical model and a numerical model for three different Reynolds numbers $(=186,929$ and 1859) as shown in Figure 2.5 from reference [37]. The same research work [37] also shows similar distributions of inlet channel, outlet channel and wall-flow velocities for different channel widths, channel lengths and wall permeabilities, showing similar trends in wall flow velocities.

Another modeling effort that compared the results from multiple models showed similar results from axially averaged values from a 3D model (KIVA3v) against a 1D model[38] as shown in Figure 2.6. One of the conclusions that was drawn from the work in reference [38] was that the velocity field in the CPF changes with the resistance encountered by the flow inside the CPF channel which is in turn a function of the geometry of the filter (such as channel length, channel width, permeability and thickness of the porous wall) and fluid flow characteristics.

Recent research work about the experimental measurement of the distribution of PM in the CPF using PM imaging techniques [39] shows the axial distribution of PM loaded in the $\mathrm{CPF}$ after a certain period of loading which shows non-uniformity of the axial distribution 

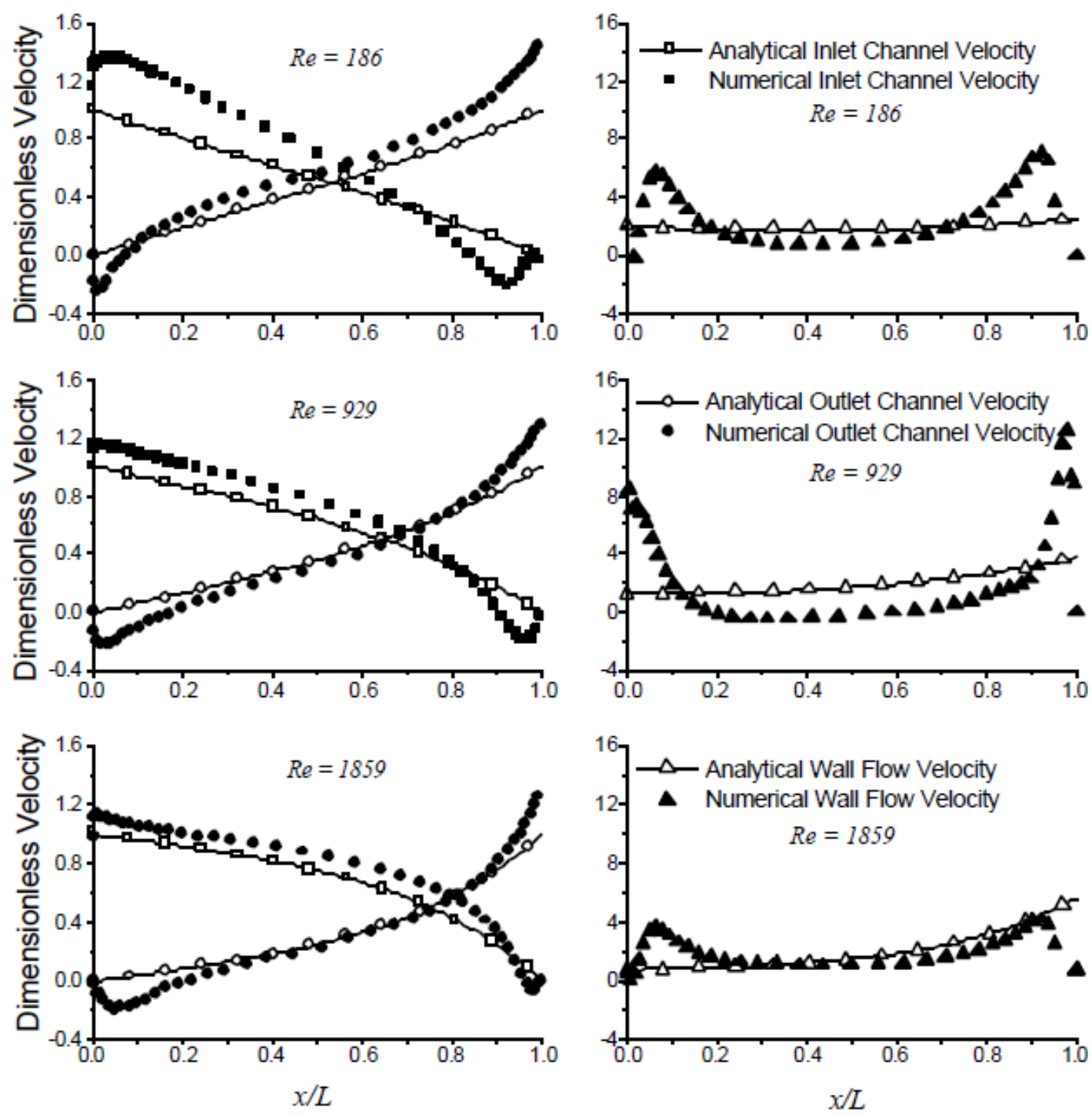

Figure 2.5: Inlet channel, outlet channel and wall velocity distributions at different Reynolds numbers[37]. - Reprinted with permission from SAE Paper No. 2002-01-1311 (C)2002 SAE International.

as later shown in chapter 5. This prompted the modeling of PM inlet rate into the axial section of the model to be considered directly proportional to the local wall-flow velocity as explained in Chapter 3. This led to significant improvement in the axial PM thickness profile prediction by the model compared to the experimental data as shown later in Chapter 5. 


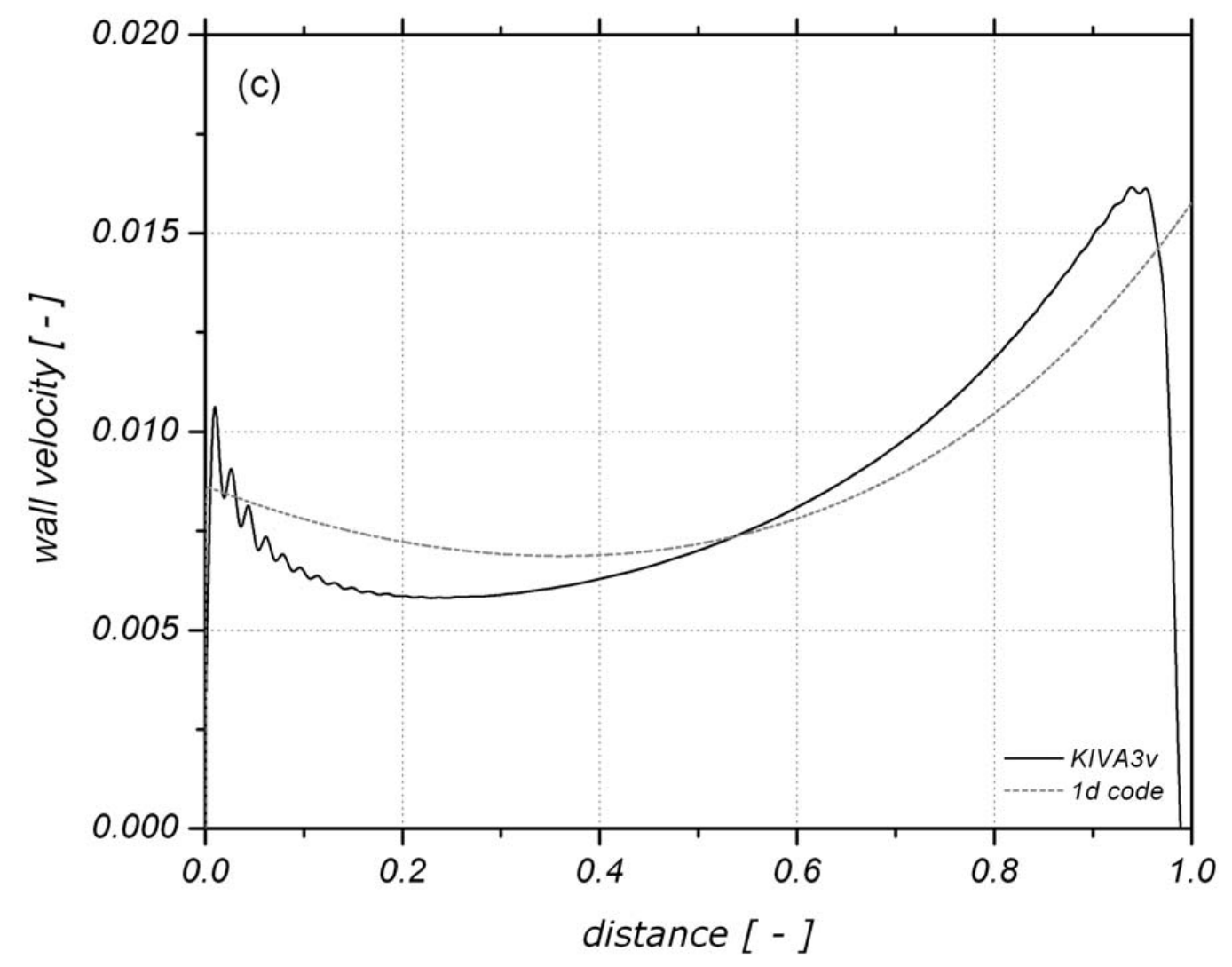

Figure 2.6: Comparison of normalized wall velocity distributions from two different models - 3D model (KIVA3v) and 1D model at the same flow conditions (space vel. $=30 \mathrm{~s}^{-1}$ ) and geometry $($ EX-80 2.66" x 4.5" 100/17, channel length $=114 \mathrm{~mm}$, channel width $=2.28 \mathrm{~mm}$, wall thickness $=$ $0.432 \mathrm{~mm}$, porosity $=48 \%$, mean pore size $=12 \mu \mathrm{m}$ ) [38]. - Reprinted with permission from SAE

Paper No. 2005-01-0963 (c)2005 SAE International.

\subsection{Oxidation of PM}

Oxidation of particulate matter in a diesel particulate filter is known to be due to two global chemical reactions: oxidation of carbon in the particulate matter with oxygen, commonly known as thermal oxidation of PM, and oxidation of carbon in the particulate matter with nitrogen dioxide $\left(\mathrm{NO}_{2}\right)$, known as $\mathrm{NO}_{2}$-assisted oxidation of PM. Thermal oxidation of PM was considered the only form of oxidation in diesel particulate filters [40-45] primarily when the DPF's did not have catalytic coatings. Models focused on oxidation of PM via 3 reactions: thermal, catalytic and $\mathrm{NO}_{2}$-assisted [13]. This framework was for the particulate matter in the PM cake layer and it extended the framework for thermal oxidation and catalytic oxidation laid out in earlier works $[46,47]$. 
Since one of the primary goals of this research work was to determine the kinetics of PM oxidation, a review of literature covering this topic was carried out. Over the years, various research works have focused on deriving the kinetics of PM oxidation. The earlier works on this topic were focused on thermal oxidation of PM with oxygen $\left(\mathrm{O}_{2}\right)$. One of the important kinetic parameters that have been reported in open literature is the activation energy of PM oxidation. Recent research works have reported activation energy values of PM oxidation as follows in Table 2.1.

Table 2.1: Activation energies of PM oxidation reported in open literature

\begin{tabular}{|c|r|l|l|}
\hline Literature & $\begin{array}{r}\text { Activation Energy } \\
{[\mathrm{kJ} / \mathrm{mol}]}\end{array}$ & Source & Oxidant \\
\hline \multirow{2}{*}[48]{} & $137 \pm 8.7$ & Diesel Soot & $\mathrm{O}_{2}$ \\
& $132 \pm 5.1$ & Model Soot & $\mathrm{O}_{2}$ \\
\hline \multirow{2}{*}[49]{} & $129 \pm 7$ & ULSD & $\mathrm{O}_{2}$ \\
& $160 \pm 3$ & $\mathrm{~B} 100$ & $\mathrm{O}_{2}$ \\
\hline \multirow{2}{*}[50]{} & 125 & ULSD & $\mathrm{O}_{2}$ \\
& 40 & ULSD & $\mathrm{NO}_{2}$ \\
\hline \multirow{2}{*}[51]{} & 103.9 & ULSD & $\mathrm{O}_{2}$ \\
& 74.8 & ULSD & $\mathrm{NO}_{2}$ \\
\hline$[52]$ & $152 \pm 5$ & ULSD & $\mathrm{O}_{2}$ \\
\hline \multirow{2}{*}[53]{} & $152 \pm 5$ & ULSD & $\mathrm{O}_{2}$ \\
& $39 \pm 3$ & ULSD & $\mathrm{NO}_{2}$ \\
\hline
\end{tabular}

A recent work on PM kinetics of diesel particulate matter [49] studied the effect of fuel type (conventional fuel versus soy-based biodiesel) on the oxidation of PM. The authors also observed that the B100-derived PM is more reactive at the same temperature compared to ULSD-derived PM. This difference was attributed to the presence of more mobile carbon (MC) in Biodiesel-derived PM compared to ULSD-derived PM. Figure 2.7 shows a relevant result obtained in this work, which shows the reaction rates (shown in log-scale) obtained from oxidation of PM generated using ULSD and B100 fuels, illustrating the higher total reaction rates for oxidation of $40 \%$ of the same initial PM at different temperatures, the only difference being the source of PM (fuel-type).

For this research work, the starting point for PM kinetics came directly from an analysis of experimental data from references [54] (for passive oxidation) and [55] (and active regeneration) based on Arrhenius plots and the total reaction rates being assumed equal to $\mathrm{NO}_{2}$-assisted in the passive oxidation experiments and thermal $\left(\mathrm{O}_{2}\right)$ in the active regeneration experiments. 


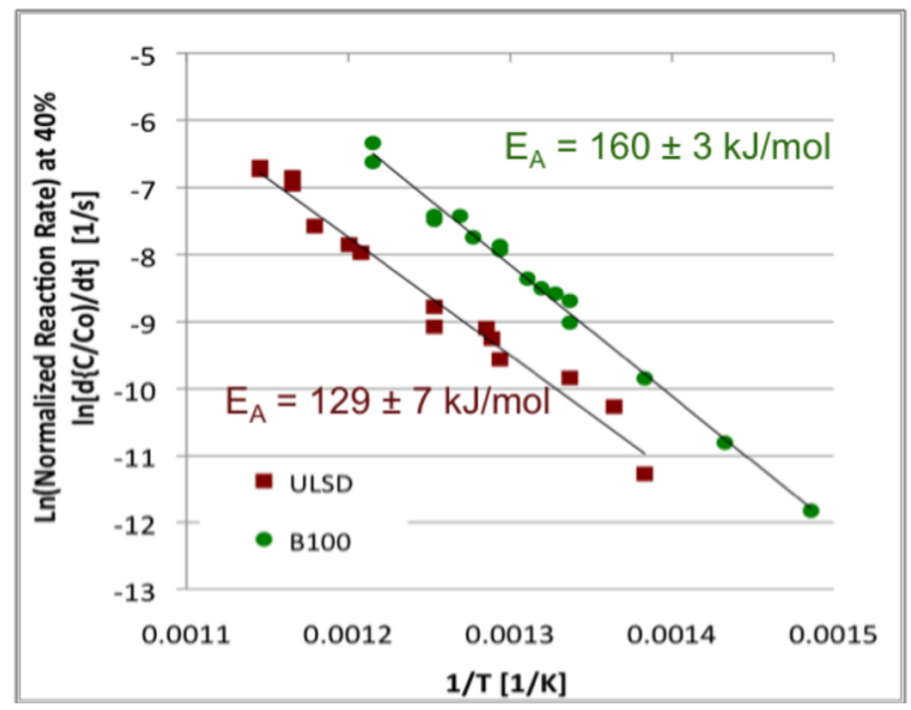

Figure 2.7: Total reaction rates obtained from oxidation of PM derived from ULSD (red squares) and B100 (green circles) fuels[49] - Reprinted with permission from the authors of SAE Paper No.

2010-01-2127 - Appendix L.

\subsection{Oxidation of Gaseous Species (Catalytic Reactions) and Back-Diffusion of $\mathrm{NO}_{2}$}

The oxidation of gaseous species $(\mathrm{HC}, \mathrm{CO}$ and $\mathrm{NO})$ in the catalytic wash-coat of a CPF was a significant improvement of the model that was carried out in the MTU CPF high-fidelity model from the existing model [56]. One of the first works about this topic was from Aristotle University Thessaloniki in Greece [52,53] which focused on the development and validation of a numerical model based on the convection-diffusion-reaction (C-D-R) framework. The reaction framework for $H C$ and $C O$ oxidation were adapted from [57] and that for $N O$ oxidation was as cited in [58]. The inclusion of this framework also implied that the diffusion of all gaseous species was automatically considered in the overall model, out of which the possible diffusion of $\mathrm{NO}_{2}$ against the direction of flow of exhaust gas could possibly be significant at certain operating conditions as shown in [53]. Such a case was illustrated in reference [53] as shown re-produced in Figure 2.8 and 2.9 for two different axial locations, one near filter entrance and one near filter exit, showing that the $\mathrm{NO}_{2}$ concentration in the PM cake layer is affected by the diffusion of $\mathrm{NO}_{2}$ produced in the PM cake layer, thus resulting in back-diffusion of $\mathrm{NO}_{2}$. To be noted here is that this work assumed that the catalytic reactions (such as oxidation of $\mathrm{NO}$ to produce $\mathrm{NO}_{2}$ ) take place in the entire thickness of the substrate wall. This could only be valid for cases where the catalyst depth of penetration is $100 \%$ of the substrate wall thickness, which is an ideal case. Another publication by the same first author [59] also described the modeling of back-diffusion of $\mathrm{NO}_{2}$ with the conclusions that the results are sensitive to the effective 
diffusivities of $\mathrm{NO}_{2}$ in the cake and wall and that further work was required to determine the effective diffusivities experimentally.

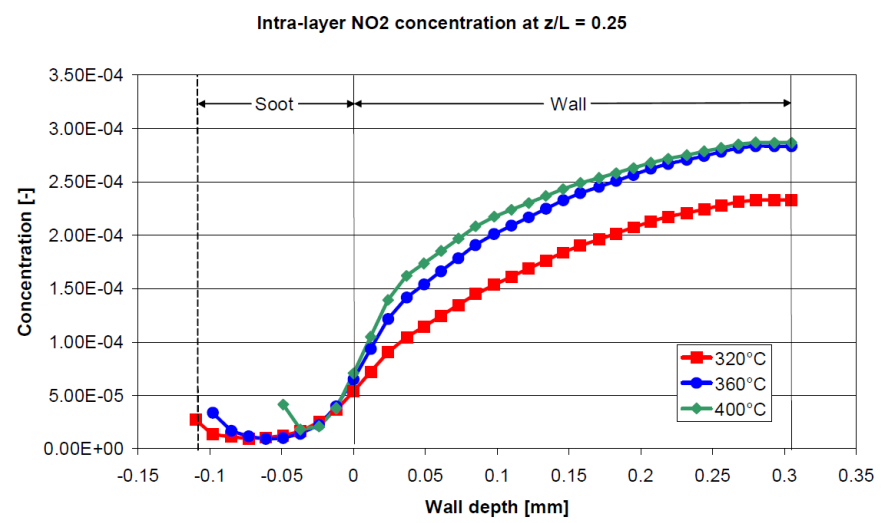

Figure 2.8: $\mathrm{NO}_{2}$ concentration profiles at three different temperatures near filter entrance $(\mathrm{z} / \mathrm{L}=$ 0.25) [53] - Reprinted with permission from SAE Paper No. 2004-01-0696 (c)2004 SAE International.

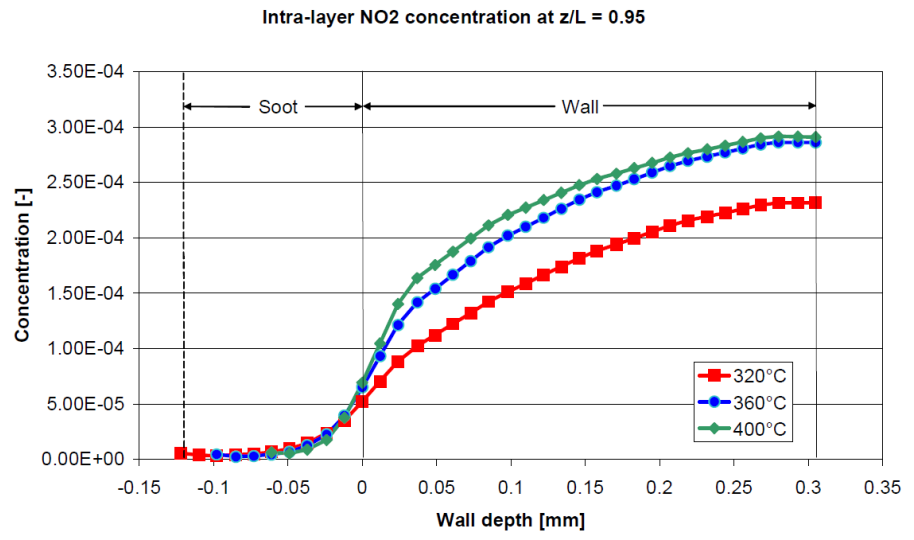

Figure 2.9: $\mathrm{NO}_{2}$ concentration profiles at three different temperatures near filter exit $(\mathrm{z} / \mathrm{L}=0.95)$ [53] - Reprinted with permission from SAE Paper No. 2004-01-0696 (c)2004 SAE International.

The reference [51] advanced the modeling of oxidation of gaseous species such as $H C$, $C O$ and $N O$ by detailing the mechanism and reactions considered as well as inhibition factor parameters. Experimental studies that quantified the effect of back-diffusion of $\mathrm{NO}_{2}$ on oxidation of PM were carried out at MTU [60,61]. The authors observed that the calculated ratio of $\mathrm{NO}_{2}$ consumed by the stoichiometric oxidation reaction with PM (assuming a stoichiometric ratio of 7.7:1) to $\mathrm{NO}_{2}$ available at the CPF inlet for the duration of some passive oxidation experiments was greater than one. This meant that the $\mathrm{NO}_{2}$ available at the CPF inlet was insufficient to fully account for the $\mathrm{NO}_{2}$ consumed by the oxidation reaction with PM. This calculation also assumed that the entire reaction of PM was with $\mathrm{NO}_{2}$ and not thermal $\left(\mathrm{O}_{2}\right)$. In some experiments, this ratio was as high as 4.5[61]. 


\subsection{Summary}

The literature reviewed in this chapter covered relevant 1-D CPF models and model results pertaining to the filtration and oxidation of PM and catalytic oxidation reactions that take place in a CPF. The main mechanisms of filtration of PM were identified as diffusion and interception. The locations of PM filtration have been identified as the substrate wall and PM cake layer. The method to determine the transition of filtration from deep-bed (porous wall) to cake filtration has been identified through the concept of transition permeability [32,33]. The catalyst sub-model has been identified as one based on the C-D-R scheme as explained in reference [53] with gaseous oxidation reactions involving $\mathrm{HC}, \mathrm{CO}$ and $\mathrm{NO}$ identified as the reactions occurring in the catalyst washcoat. Also, the mechanisms for oxidation of PM were identified as $\mathrm{NO}_{2}$-assisted and thermal

$\left(\mathrm{O}_{2}\right)$, taking place in the substrate wall and the PM cake layer. The flow rate of PM into each axial section of the CPF has been determined to be modeled directly proportional to the wall-flow velocity at the specific axial location.

The literature review that was carried out thus resulted in the identification of sub-models of the MTU CPF high-fidelity model that needed to be improved or developed broadly as:

- Area conservation of inlet channel due to PM cake layer deposition,

- Compressible flow of exhaust gas,

- The catalyst sub-model,

- PM oxidation sub-model consistent with the catalyst sub-model, and

- Sub-model for determining packing density of PM in substrate wall.

The next chapter describes the MTU CPF high-fidelity model and its sub-models. 


\section{Development of THE CPF ModeL ${ }^{1}$}

The MTU CPF high-fidelity model ("CPF model" or simply "the model" hereafter) is a computer model that can be used to simulate processes occurring in a catalyzed particulate filter when exhaust gas mixture passes through the CPF. The engine-out (and thus, CPF inlet) conditions of the exhaust gas mixture can be time-varying. A set of experimental data is needed to determine the values of the various input parameters to the model. When calibrated and validated, the model can be used to predict several relevant output variables that relate to the performance of the CPF without conducting extensive experiments on the physical device, which can bring down costs associated and development time. This chapter provides a detailed description of the MTU CPF high-fidelity model version 4.0, which was developed at MTU as part of this research work.

\subsection{Brief Development History}

The 1-dimensional CPF model was written originally by Cuong T. Huynh as part of a Masters' thesis work [62,63] (Version 1), based on the framework set by Ed Bissett [8] and Konstandopoulos et al [11]. This model code was re-written (version 2) which formed the basis of any subsequent development of the model.

The version of CPF model code used as a starting point for developing version 4.0 model was obtained from Hasan Mohammed, who worked on the model as part of a Master's thesis work [64] and later described in reference [56] (version 3). This model had the following features:

1. Filtration of PM by PM cake layer and substrate wall,

2. Oxidation of PM in the substrate wall and PM cake layer due to thermal and $\mathrm{NO}_{2}$-assisted reactions, and

\footnotetext{
${ }^{1}$ Parts of the material contained in this chapter have been published, or are currently under consideration for publication by SAE International.
} 


\section{2-layer framework for the oxidation of PM cake layer.}

One of the objectives behind developing the CPF model was to be able to simulate active regeneration by fuel injection before a DOC placed upstream of the CPF. The other objective was to enable the CPF to simulate passive oxidation under normal engine operation conditions. The most important addition that was required to be made to the CPF model was a catalyst submodel, able to predict oxidation of gaseous species such

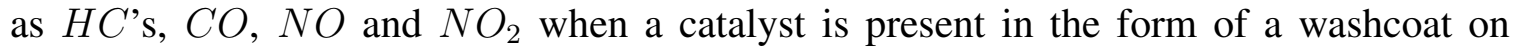
or in the substrate wall of the particulate filter. Also, the wall energy balance needed to include energy release source terms from these chemical reactions such that during active regeneration, a positive energy release occurs in the catalyst and and hence results in an increase in the wall temperature (exotherm) which is significant compared to the overall temperatures. A review of published literature led to a catalyst model which used a mass balance of individual chemical species in the inlet channel, PM cake layer, catalyst washcoat and substrate wall (hereafter called 'cake+cat+wall') and outlet channel to solve for concentrations of the chemical species [53]. The equations and solution procedure that was followed to develop the catalyst submodel is explained later in sub-section 3.4.5 of this chapter.

One of the major assumptions that was used in arriving at the gaseous velocity solution that was used in the previous version of the CPF model was incompressible (constant density) flow. In other words, the density of the exhaust gas as it passes through the inlet channel, cake+cat+wall and outlet channel control volumes was assumed to be constant. It is known that even given ideal gas behavior, the variation in density of exhaust gas can be significant from the inlet to the outlet of the CPF. Therefore, it was decided to rewrite the solution of the gaseous velocity field in the CPF such that density of the exhaust gas is considered to be variable (compressible flow). The densities of exhaust gas mixture are calculated at each axial location as functions of local pressures, temperatures and equivalent molecular weights according to the ideal gas law. Accordingly, the system of equations to be solved were re-written according to the derivation described in Appendix B.

During the model development phase of this work, it was also observed that the existing literature on 1-D models does not consider the dependence of the rate at which PM is carried to each axial discretized control volume to the wall-flow velocity. Wall-flow velocity solution obtained at clean state shows variable velocity. Therefore, in order to conserve mass of the exhaust gas mixture, it would be accurate to assume that the mass flow rate of exhaust gas into each axial wall face is also variable (since mass flow rate of gas is directly proportional to velocity of the gas, density of the gas and cross-sectional area of flow of the gas). Thus, for a given constant CPF inlet PM concentration, the rate of PM inlet to each cake+cat+wall control volume would be directly proportional to the wall-flow velocity. This was implemented in the PM mass balance solution in the calculation of PM inlet rate into the PM cake layer at each time-step. This method affected the growth of PM 
cake layer and the PM cake layer thickness that was predicted by the model at the end of a loading experiment, resulting in better agreement with experimental data as is shown later in Chapter 5.

\subsection{Overview of the Model}

The salient features of the CPF model as developed in this thesis and desribed in reference [65] are:

- PM filtration in cake layer and substrate wall,

- Catalytic reactions of gaseous species - hydrocarbons (represented by n-dodecene $\left.\left[\mathrm{C}_{12} \mathrm{H}_{24}\right]\right), \mathrm{CO}$ and $\mathrm{NO}$ with the inclusion of reversible reaction of $\mathrm{NO}$ with $\mathrm{O}_{2}$ to form $\mathrm{NO}_{2}$,

- Energy release to account for the experimentally observed axial increase in temperature of the CPF substrate and hence the temperature of exhaust gas mixture in the axial direction (majority of which is due to oxidation of HC's and some due to oxidation of PM),

- Mechanism for back-diffusion of $\mathrm{NO}_{2}$ which would 're-cycle' $\mathrm{NO}_{2}$ during passive oxidation conditions,

- PM oxidation in cake layer and wall,

- Variable wall PM properties during deep-bed filtration,

- Local PM flow rate into each axial element proportional to wall-flow velocity at the specific location, and

- Convergent solution that is inclusive of all major variables solved for in the model.

The CPF model is based on a single channel representation of the filter. Figure 3.1 shows a schematic of the single channel, which consists of a single inlet channel, PM cake layer, catalyst washcoat and substrate wall and an outlet channel. This framework is used to develop the governing equations to solve all the variables at each location (axial for all variables and transverse in the case of species concentrations) of the CPF. The model is still considered 1-dimensional since the governing equations that are solved to obtain the model outputs are differential equations in one spatial dimension (' $x$ ' or ' $y$ '). In the case of a 'clean' CPF, the PM cake layer and PM in the wall are not present in the CPF. The inlet channel of the CPF is coated with a thin layer of catalyst in the form of a washcoat. This 
can form either a separate sub-layer on top of the washcoat or can penetrate the pores of the substrate wall, depending on the method of catalyst washcoat application. The width of the inlet channel, thus, is different from that of the outlet channel due to the presence of the catalyst washcoat and varying thickness of PM cake layer deposited on the catalyst washcoat.

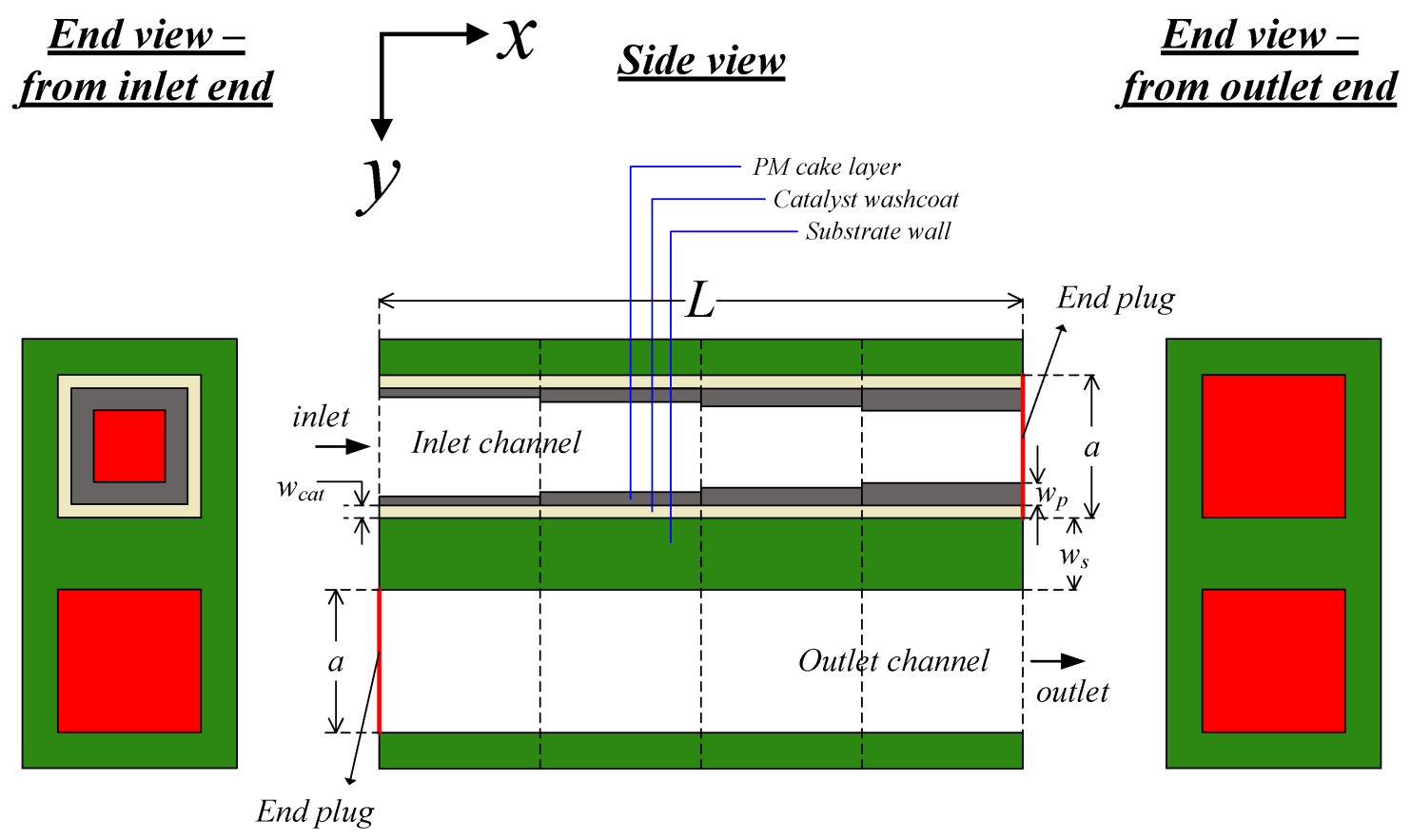

Figure 3.1: Schematic representation of the single channel approach used in the development of the CPF model. Reprinted with permission from SAE Paper No. 2013-01-1574 (C)2013 SAE International.

The inlet channel, cake+cat+wall and outlet channel control volumes are discretized in the axial $(x)$ direction into a user-specified number of discretizations. Figure 3.2 shows a schematic of the axially discretized control volumes of the inlet channel, cake+cat+wall and outlet channel and the geometric dimensional variables involved.

The axially discretized cake+cat+wall control volumes are further divided in the transverse $(y)$ direction as shown in Figure 3.3 for application of the species mass conservation equations. 


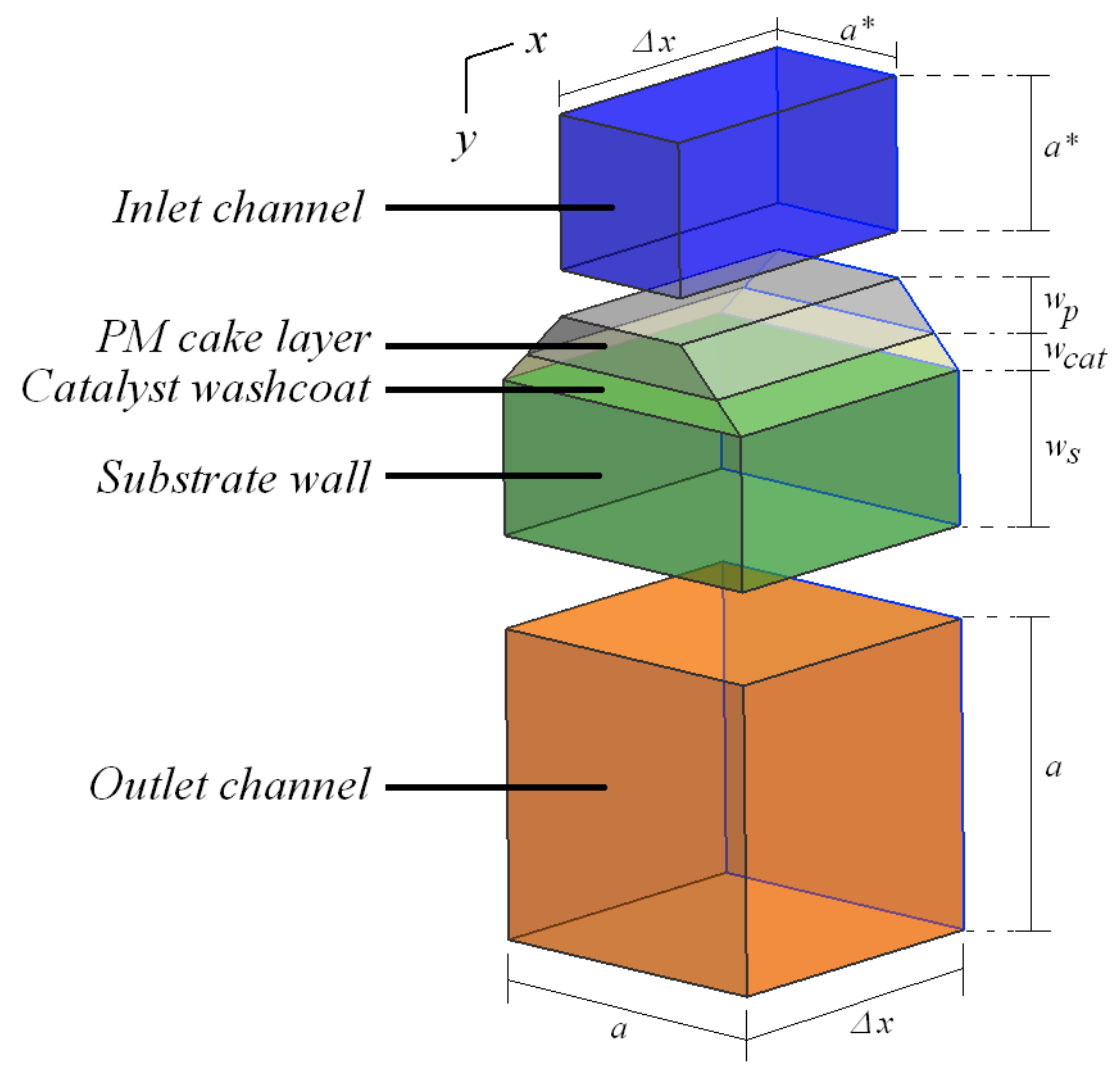

Figure 3.2: Schematic representation of the discretized inlet channel, PM cake layer, catalyst washcoat, substrate wall and outlet channel control volumes 


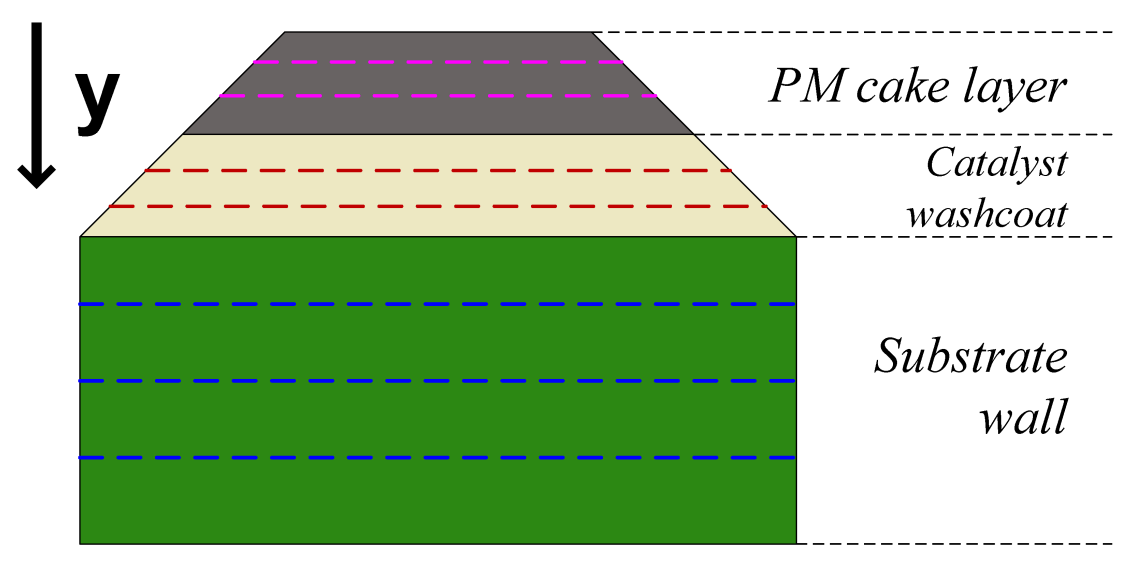

Figure 3.3: Schematic representation of the PM cake layer, catalyst washcoat and substrate wall (cake+cat+wall) control volume discretized in the transverse (y) direction. In this case, number of discretizations in the PM cake layer $=3$, number of discretizations in the catalyst washcoat $=3$, and number of discretizations in the substrate wall $=4$. Reprinted with permission from SAE Paper No. 2013-01-1574 (c)2013 SAE International. 


\subsection{Structure of the Model Code}

The computer code for the CPF model is written in the FORTRAN77 programming language. The overall structure of the program (model code) as shown in Figure 3.4 consists of a main body and several subroutines that the main body accesses through call statements. A brief procedure of how to compile and run simulations with the model is given in Appendix F.

The main body of the program consists of an outer time-loop as well as an inner iteration loop. All simulations start from a start-time equal to zero. The total simulation time (specified by the user as an input into the model) is initially divided equally into a number of steps in time. First, the model code reads all specified inputs and sets the initial conditions for the problem. Then the model enters the time-loop. Each step of the outer time-loop of the model code is called a time-step, where the model advances from the previous time to the current time according to the time-step specifications that the user has input into the model and current time-step calculations that the model carries out (this is explained later in section 3.4.8 of this chapter).

At each time-step, the model code goes through at least two iterations of the inner loop. During each of these iterations, the different subroutines of the model code calculate current values of different variables and return those values to the main body of the model code. At the end of the second iteration within the inner loop, primary variables that are calculated are then selected to check for convergence against user-specified tolerance values. The logic for convergence check is explained later in section 3.4.8 of this chapter. Depending on whether convergence is attained or not, the solution is either advanced or more iterations within the inner loop are carried out.

When advancing from one time-point to the next, the model code also checks the current time against a calculated value of user-specified value of time at which model-predicted data is to be output. For example, if the user-specified value of an input variable called ndatafreq is 2, that means that the output is written at the first step (regardless of what the value of ndatafreq is) and every $2^{\text {nd }}$ time-step, i.e., if initial time-step specified is 10 seconds, at time equal to 20 seconds, 40 seconds, 60 seconds and so on. If the time-step gets sub-divided to, say 2 seconds after this point, then the data is written out at time equal to $64,68,72$ seconds and so on. When the total simulation time has been reached, the solution exits the time-loop, prints outputs to output files and exits. Additional flowcharts for the individual calculations are shown in Figure 3.4 and expanded in Appendix E. 


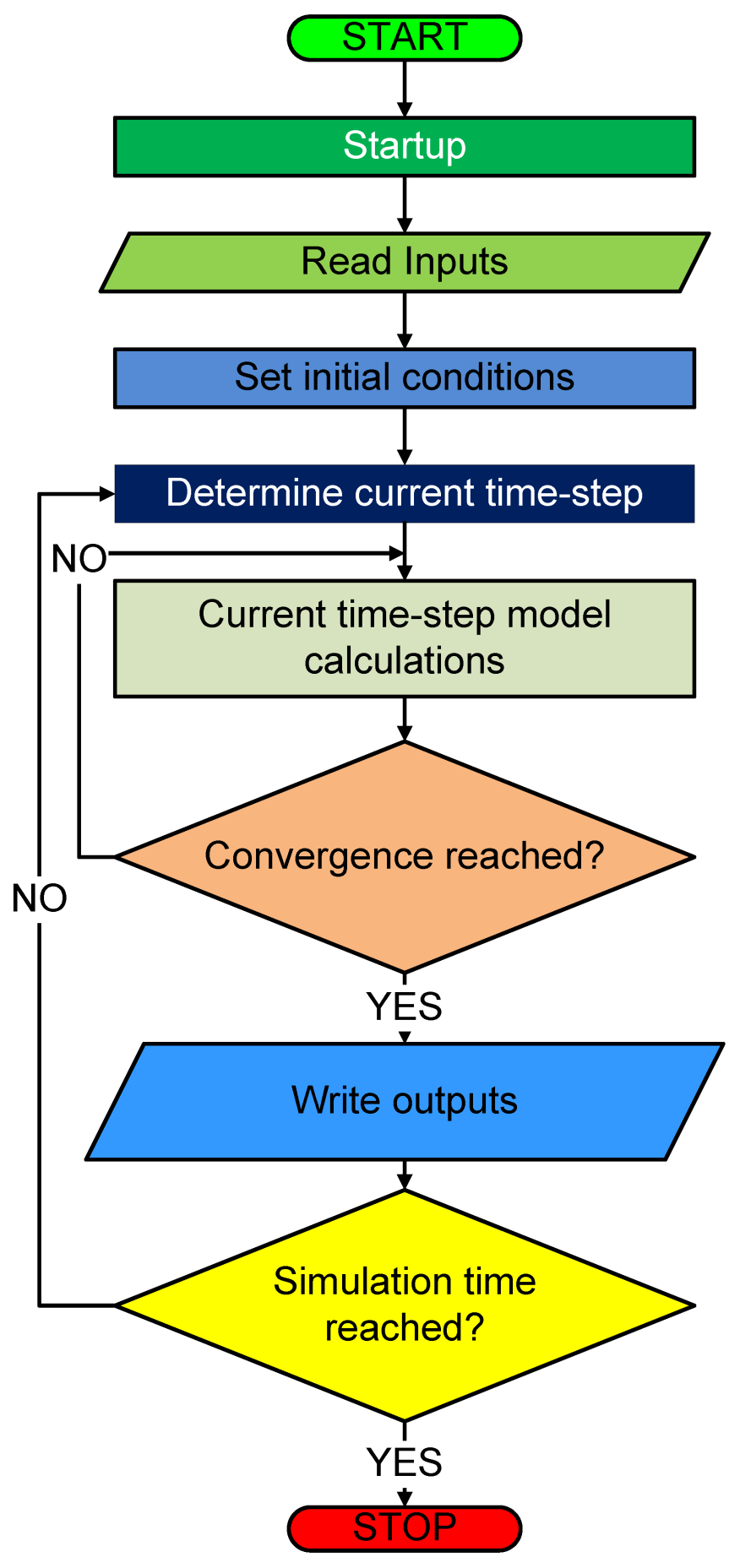

Figure 3.4: Flowchart showing the overall structure of the CPF model 


\subsection{Submodels and Numerical Solution Procedure}

The major variables solved for in the CPF model are exhaust gas densities $\left(\rho_{m}\right)$, exhaust gas velocities $\left(v_{m}\right)$, absolute pressures $\left(P_{m}\right)$, absolute temperatures $\left(T_{m}\right)$ and concentrations (mole fractions) of chemical species $\left(Y_{m, i}\right)$. Here, the subscript $(m)$ can be (1), (2) or $(w)$, denoting inlet channel, outlet channel and cake+cat+wall respectively. In the case of mole fractions of individual chemical species, the cake+cat+wall mole fractions are simply denoted as $\left(Y_{i}\right)$ where $i$ is the index of the chemical species.

This section describes the different submodels in the CPF model version 4.0 as shown in Figure E.1 and the numerical solution of variables that are solved for within these various submodels. Each submodel is a separate module called 'subroutine' in FORTRAN77 programming language unless otherwise stated.

\subsubsection{Calculating Thermophysical Properties of the Exhaust Gas}

The thermophysical properties of the exhaust gas are calculated by a subroutine in the FORTRAN model code called 'thermomixture_1d', which is invoked (via a 'call' statement) at each sub-iteration of every time-step as can be seen from the Figure E.1 (box titled 'calculate exhaust gas properties'). The following sub-sections give the equations and methods used to calculate these properties.

\section{Density}

Equations of state are employed to calculate the density of the exhaust gas mixture at various locations in the particulate filter. The assumption here is that exhaust gas mixture behaves like an ideal gas of the same equivalent molecular weight. These can be expressed as:

$$
\rho_{e x h, m}=\frac{P_{m}(M W)_{e x h, m}}{\bar{R} T_{m}}, m=1,2, w
$$

where $\rho_{e x h, m}$ is the density of the exhaust gas mixture, $P_{m}$ is the absolute pressure of the exhaust gas mixture, $(M W)_{e x h, m}$ is the molecular weight of the exhaust gas mixture, $\bar{R}$ is the universal gas constant and $T_{m}$ is the absolute temperature of the exhaust gas mixture. The subscripts 1,2 and $w$ designate the inlet channel, outlet channel and cake+cat+wall control volumes respectively. Here, $P_{w}=\frac{P_{1}+P_{2}}{2}$ is the assumed absolute pressure of the gas in the cake+cat+wall control volume. Also, the absolute temperature of the gas in the cake+cat+wall control volume is assumed to be equal to the absolute temperature of the cake+cat+wall control volume $\left(T_{w}\right)$. 
Density of the exhaust gas mixture as it passes through the inlet channel, cake+cat+wall and outlet channel control volumes is calculated using the ideal gas equation of state (3.1). This calculation needs absolute pressures, absolute temperatures and equivalent molecular weights at each of these locations. Therefore, in the first sub-iteration at every time-step of the simulation, values from previous time-steps of the variables are used since these are the only available values. From the second sub-iteration onwards, these variables are updated by the corresponding values from the previous sub-iteration of the same time-step.

Also, molecular density of the exhaust gas mixture (expressed in $\left[\frac{\mathrm{kmol}}{\mathrm{m}^{3}}\right]$ ) as it passes through the inlet channel, cake+cat+wall and outlet channel control volumes is calculated from equation (3.1) as:

$$
\bar{\rho}_{e x h, m}=\frac{\rho_{e x h, m}}{(M W)_{e x h, m}}
$$

Molecular density of the exhaust gas mixture is used later in the conversion of mole fractions of individual chemical species to molar concentrations as explained later in section 3.4.5.

\section{Molecular Weight}

The molecular weight of the exhaust gas mixture $\left(M W_{m i x}\right)_{m}$ can be calculated knowing the mole fractions of all gaseous components $i$ as follows:

$$
(M W)_{e x h, m}=\sum_{i=1}^{n_{s p}}\left(Y_{i}\right)_{m}(M W)_{i}
$$

where $\left(Y_{i}\right)_{m}$ are the mole fractions of the individual chemical species, $(M W)_{i}$ are the molecular weights of the individual chemical species that constitute the exhaust gas mixture and $m$ is the subscript denoting location (inlet channel $=1$, outlet channel $=2$, cake+cat+wall $=w)$. 


\section{Specific Heat}

Specific heat of the exhaust gas mixture $c_{p}$ is calculated using the relation as follows[66]:

$$
c_{p}=\frac{1}{(M W)_{\text {mix }}} \sum_{i} Y_{i}\left[A_{i}+B_{i}\left(\frac{\frac{C_{i}}{T}}{\sinh \left(\frac{C_{i}}{T}\right)}\right)^{2}+D_{i}\left(\frac{\frac{E_{i}}{T}}{\cosh \left(\frac{E_{i}}{T}\right)}\right)^{2}\right]
$$

where $M W$ is the molecular weight of the exhaust gas mixture, $Y_{i}$ are the mole fractions of the constituent chemical species of the exhaust gas mixture and the coefficients $A_{i}, B_{i}$, $C_{i}, D_{i}$ and $E_{i}$ used for calculating specific heats are given in Table G.1 in Appendix G.

\section{Dynamic Viscosity}

The dynamic viscosity of the exhaust gas mixture $\mu_{m i x}$ is calculated from the individual dynamic viscosities $\mu_{i}$ of the chemical species constituting the exhaust gas mixture as [66]:

$$
\mu_{m i x}=\sum_{i=1}^{n_{s p}} Y_{i} \frac{\mu_{i}}{e_{i}}
$$

In equation (3.5), $\mu_{i}$ are calculated as [66]:

$$
\mu_{i}=\frac{A_{i} T^{B_{i}}}{1+\frac{C_{i}}{T}+\frac{D_{i}}{T^{2}}}
$$

where the constant coefficients $A_{i}, B_{i}, C_{i}$ and $D_{i}$ used in equation (3.6) for the different chemical species assumed to constitute the exhaust gas mixture in the CPF model version 4.0 are as given in Table G.2 in Appendix G.

Also, the coefficients $e_{i}$ in equation (3.5) are calculated as [66]:

$$
e_{i}=\sum_{j=1}^{n_{s p}} Y_{i} f_{i, j}
$$

where:

$$
f_{i, j}=\left[\left(1+\left(\frac{\mu_{i}}{\mu_{j}}\right)^{1 / 2}\right)\left(\frac{M W_{j}}{M W_{i}}\right)^{1 / 4}\right]^{2}\left[\frac{1}{\left(1+\frac{M W_{i}}{M W_{j}}\right)^{1 / 2} \sqrt{8}}\right]
$$




\section{Thermal Conductivity}

Thermal conductivity of the exhaust gas mixture $k_{m i x}$ is calculated as [66]:

$$
k_{\text {mix }}=\sum_{i=1}^{n_{s p}} Y_{i} \frac{k_{i}}{e_{i}}
$$

where:

$$
k_{i}=\frac{A_{i} T^{B_{i}}}{1+\frac{C_{i}}{T}+\frac{D_{i}}{T^{2}}}
$$

The coefficients $A_{i}, B_{i}, C_{i}$ and $D_{i}$ used in Equation (3.10) for the chemical species assumed to constitute the exhaust gas mixture in the CPF model version 4.0 are as given in Table G.3 in Appendix G. Also, the coefficients $e_{i}$ are calculated using Equation (3.7).

\section{Convection Heat Transfer Coefficient}

The heat transfer coefficients $h_{1}$ and $h_{2}$ that appear in Equations (3.25), (3.26) and (3.27) are calculated according to the relation $[8,66]$ :

$$
h_{m}=\frac{N u}{d_{m}}\left(c_{p, \text { mix }}+358.80876\right) \mu_{m i x}
$$

where the subscript $m$ can be 1 or 2 denoting inlet channel or outlet channel respectively, and the corresponding length scales $d_{m}$ are $a^{*}$ or $a$ respectively.

\subsubsection{Packing Density of PM in the Wall}

Packing density of particulate matter in the substrate wall $\left(\rho_{p w}\right)$ (or simply "wall packing density" [13]) is used to calculate the increase of unit collector diameter with PM deposition in the wall unit collector expressed mathematically as shown in Equation 3.62. The original assumption that $\rho_{p w}$ is a (user-defined) constant was modified in the current $\mathrm{CPF}$ model. By matching the increase of model-predicted pressure drop to experimental values during deep-bed filtration regime where no PM cake layer is present, a desired relation of wall packing density was detremined which could be modeled empirically by a linear relation with respect to the total mass of PM present in the wall, expressed as:

$$
\rho_{p w}=C_{1, \rho_{p w}} m_{w a l l}+C_{2, \rho_{p w}}
$$

where $C_{1, \rho_{p w}}$ and $C_{2, \rho_{p w}}$ are user-specified constants and $m_{\text {wall }}$ is the total mass of PM retained in the wall. Figure 3.5 shows the model-predicted pressure drop using a 
constant wall packing density and using the relation shown in Equation 3.12 compared to experimental data showing the improvement in model-predicted pressure drop during the deep-bed filtration regime.

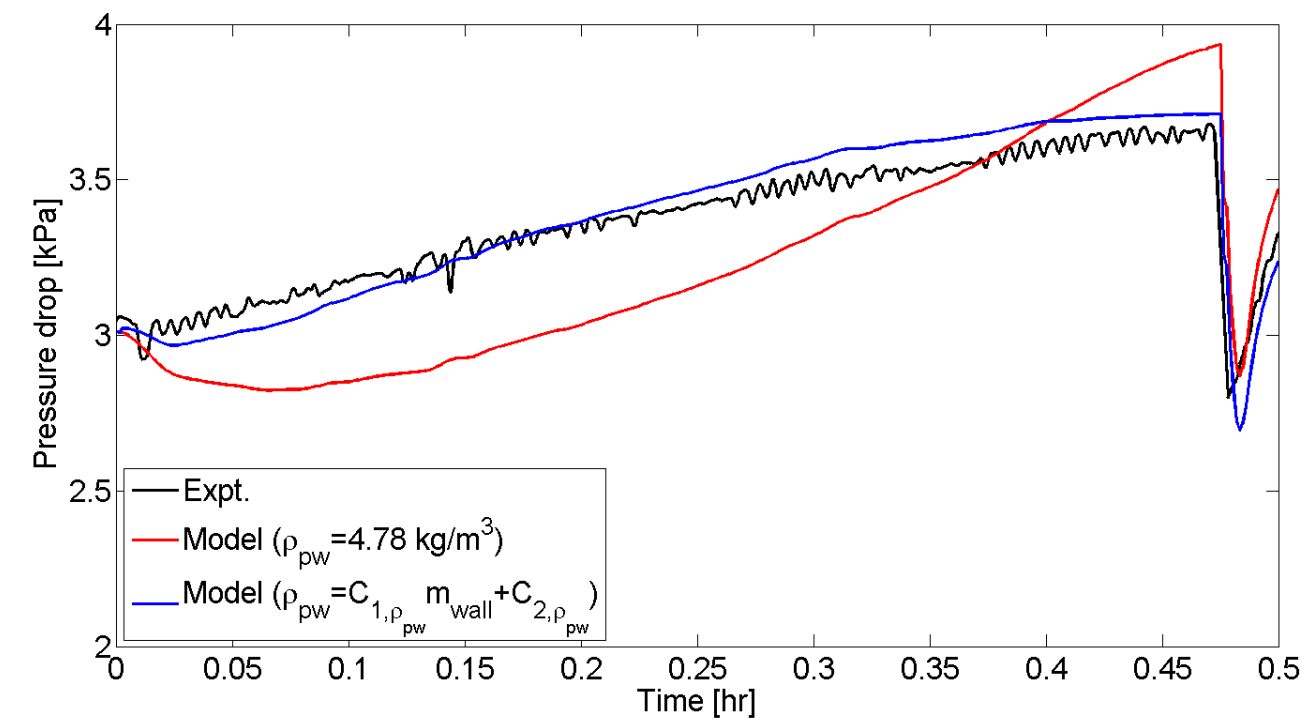

Figure 3.5: Model-predicted CPF pressure drop with constant and variable wall packing density compared to experimental pressure drop

\subsubsection{Solving the Gaseous Velocity Field}

\section{Equations of Conservation of Mass of Exhaust Gas Mixture}

Mass conservation equations used in the inlet channel, outlet channel and wall of the model are derived from conservation of mass of the exhaust gas mixture as it passes through different locations in the CPF. A detailed derivation of mass conservation equations in the inlet channel, cake+cat+wall and outlet channel control volumes are shown in Appendix A.

The steady form of conservation of mass of the exhaust gas mixture in a control volume in the inlet channel of the particulate filter can be expressed mathematically as:

$$
\frac{d}{d x}\left(\rho_{1} v_{1}\right)=-\frac{4}{a}\left(\frac{a}{a^{*}}\right)^{2} \rho_{w} v_{w}
$$

where $v_{1}$ is the velocity of the exhaust gas mixture in the inlet channel and $a$ is the width of the clean inlet channel before application of catalyst washcoat. The effective width of the inlet channel $a^{*}$ is calculated as $a^{*}=a-2 w_{c a t}-2 w_{p}$ at a given axial location. Figure 3.6 is a diagram showing how the effective width of the inlet channel can be calculated knowing 
the clean channel width $(a)$, catalyst washcoat thickness $\left(w_{c a t}\right)$ and instantaneous PM cake layer thickness $\left(w_{p}\right)$. Note that $a^{*}$ is a function of axial location since $w_{p}$ is a function of the axial location.

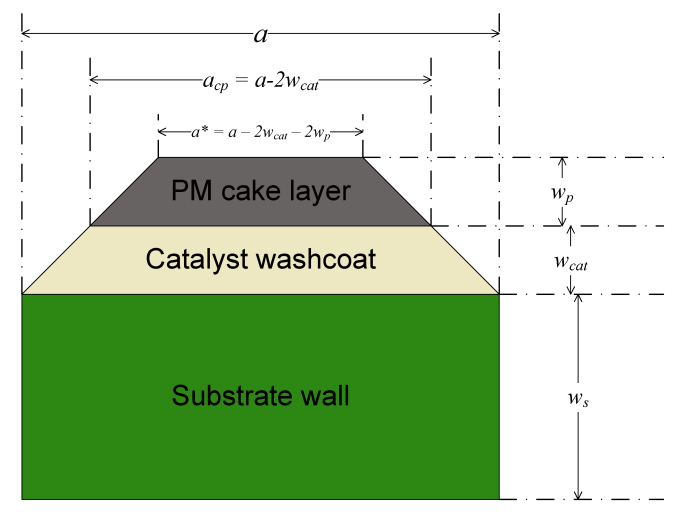

Figure 3.6: Diagram showing the calculation of effective inlet channel width

The quasi-steady form of the conservation of mass of the exhaust gas mixture as it passes through the PM cake layer, catalyst washcoat and substrate wall can be expressed as:

$$
\rho_{w} v_{w}^{*} a^{*} \Delta x=\rho_{w} v_{w} a \Delta x
$$

where $v_{w}^{*}$ is the 'entry velocity' of the exhaust gas mixture as it enters the cake+cat+wall control volume, $v_{w}$ is the 'exit velocity' of the exhaust gas, $\rho_{w}$ is the density of the exhaust gas mixture (assumed constant) in the cake+cat+wall control volume and $\Delta x$ is the discretized dimension of each cake+cat+wall control volume in the axial $(x)$ direction, which can be expressed mathematically as $\Delta x=\frac{L}{j}$ where $j$ is the number of discretizations in the axial direction and $L$ is the total length of the CPF.

The steady form of conservation of mass of the exhaust gas mixture in a control volume in the outlet channel of the particulate filter can be expressed mathematically as:

$$
\frac{d}{d x}\left(\rho_{2} v_{2}\right)=\frac{4}{a} \rho_{w} v_{w}
$$

where $v_{2}$ is the velocity of the exhaust gas mixture in the outlet channel. 
Steady form of the conservation of axial momentum of the exhaust gas mixture as it passes through the inlet channel is derived in Appendix B and is:

$$
\frac{d}{d x}\left(\rho_{1} v_{1}^{2}\right)=-\frac{d P_{1}}{d x}-F \frac{\mu_{1} v_{1}}{a^{2}}\left(\frac{a^{*}}{a}\right)^{2}
$$

where $P_{1}$ is the absolute pressure of the exhaust gas mixture in the inlet channel, $F$ is the fanning friction factor (and assumed constant $=28.454$ as discussed in reference [11]) and $\mu_{1}$ is the dynamic viscosity of the exhaust gas mixture in the inlet channel. This equation results from balancing the convection of axial momentum (LHS term in Equation (3.16)) to the pressure forces (first term on RHS of Equation (3.16)) and frictional forces (second term in Equation (3.16)) that are counteracting this convection.

Steady form of the conservation of axial momentum of the exhaust gas mixture as it passes through the outlet channel is derived in Appendix B) and is:

$$
\frac{d}{d x}\left(\rho_{2} v_{2}^{2}\right)=-\frac{d P_{2}}{d x}-F \frac{\mu_{2} v_{2}}{a^{2}}
$$

where $P_{2}$ is the absolute pressure of the exhaust gas mixture in the outlet channel and $\mu_{2}$ is the dynamic viscosity of the exhaust gas mixture in the outlet channel.

\section{Numerical Solution Procedure}

Solution of the gaseous velocity field to obtain the velocity of the exhaust gas mixture at different axial locations in the inlet channel, substrate wall and outlet channel is carried out by solving a boundary value problem involving a system of two first order ordinary differential equations. Appendix B shows the derivation of the equations used for solving for the gaseous velocity field in the particulate filter. The system of Equations (B.34) is reproduced here for convenience.

$$
\begin{aligned}
\frac{d N}{d x} & =G \\
\frac{d G}{d x} & =\frac{1}{A_{1}}\left[G\left(A_{2}+A_{3}+A_{4}\right)+N^{2}\left(A_{5}+A_{6}\right)+N\left(A_{7}+A_{8}\right)\right. \\
& \left.+M^{2}\left(A_{9}\right)+M\left(A_{10}\right)\right]
\end{aligned}
$$


where:

$$
\begin{aligned}
& A_{1}=-\frac{a}{4} \gamma \\
& A_{2}=-\frac{2 M}{\rho_{1}} \\
& A_{3}=\frac{2 N(1-H)}{\rho_{1}} \\
& A_{4}=\frac{a}{4} \frac{d \gamma}{d x} \\
& A_{5}=-\frac{1}{\rho_{1}} \frac{d H}{d x} \\
& A_{6}=-\frac{1-H}{\rho_{1}^{2}} \frac{d \rho_{1}}{d x} \\
& A_{7}=\frac{2 M}{\rho_{1}^{2}} \frac{d \rho_{1}}{d x} \\
& A_{8}=-\frac{F\left(\mu_{1}+H \mu_{2}\right)}{\rho_{1}\left(a^{*}\right)^{2}} \\
& A_{9}=-\frac{1}{\rho_{1}^{2} \frac{d \rho_{1}}{d x}} \\
& A_{10}=\frac{F \mu_{1}}{\rho_{1}\left(a^{*}\right)^{2}}
\end{aligned}
$$

and

$$
\begin{aligned}
\gamma & =\frac{\mu_{w}}{\rho_{w}}\left(\frac{w_{p}}{k_{p}}+\frac{w_{s}}{k_{s}}\right) \\
H & =\frac{\rho_{1}}{\rho_{2}} \\
M & =\frac{\dot{m}_{\text {in }}}{n_{\text {cells }}(a)^{2}} \\
N & =\rho_{2} v_{2}
\end{aligned}
$$

where $\mu_{w}$ is the dynamic viscosity of the exhaust gas mixture in the cake+cat+wall control volume, $\rho_{w}$ is the density of the exhaust gas mixture in the cake+cat+wall control volume, $w_{p}$ is the thickness of the PM cake layer, $k_{p}$ is the instantaneous permeability of the PM cake layer, $w_{s}$ is the thickness of the substrate wall, $k_{s}$ is the instantaneous permeability of the substrate wall, $\dot{m}_{i n}$ is the CPF inlet mass flow rate and $n_{\text {cells }}$ is the number of inlet channels in the CPF $\left(=\frac{\frac{\pi D^{2}}{4}}{2 a^{2}}\right)$. In order to solve for the dependent variables $N$ and $G$ as given in the system of Equations (3.18), information that is available about the boundary 
values for outlet channel is as follows:

$$
\begin{array}{lr}
\left.N\right|_{x=0}=0 & \text { \}no-slip condition due to end-plug } \\
\left.N\right|_{x=L}=M & \text { \} from mass conservation }
\end{array}
$$

The shooting method in reference [67] is employed to convert the problem from a boundary value problem to an initial value problem and a 'marching' method is used to solve the system of equations. A $4^{\text {th }}$-order Runge-Kutta method [67] is used in this case to march the solution of Equations (3.18a) and Equation (3.18b) simultaneously through the domain $(x=0)$ to $(x=L)$. The value of $G$ at $(x=0)$ is initially assumed to be equal to $\frac{M}{L}$. At $(x=L)$, the value of a discrepancy function defined as $f=\left.N\right|_{x=L}-M$ is evaluated and a secant method is employed to find the ideal value of $\left.G\right|_{x=0}$ such that $f \rightarrow 0$.

Knowing the solution of $N$, we can recover outlet channel velocity $v_{2}$ as:

$$
v_{2}=\frac{N}{\rho_{2}}
$$

Then, from equation (B.12) in Appendix B, we can find:

$$
v_{1}=\frac{M-N}{\rho_{1}}
$$

Also, from equation (B.1b) in Appendix B, it follows that:

$$
v_{w}=\frac{a}{4} \frac{\frac{d N}{d x}}{\rho_{w}}
$$

Hence, gaseous velocities in the inlet channel $\left(v_{1}\right)$, outlet channel $\left(v_{2}\right)$ and wall $\left(v_{w}\right)$ can be determined knowing the densities of the exhaust gas from the equation of state, Equation (3.1).

\subsubsection{Solving the Temperature Field}

\section{Equations of Conservation of Energy of Exhaust Gas Mixture and Substrate Wall}

The steady form of energy conservation is employed to solve for the temperature of the exhaust gas mixture in the inlet channel. Convection of energy along the axial direction is considered. Axial conduction of energy through the exhaust gas mixture is neglected since convection via fluid flow is known to be the dominant mechanism of energy transport at all conditions. The steady-state energy balance governing equation can be mathematically 
expressed as:

$$
\frac{d T_{1}}{d x}=-\frac{4}{a}\left(\frac{a}{a^{*}}\right) \frac{h_{1}}{\rho_{1} v_{1} c_{p 1}}\left(T_{1}-T_{w}\right)
$$

where $T_{1}$ is the absolute temperature of the exhaust gas mixture in the inlet channel, $h_{1}$ is the heat transfer coefficient for convective heat transfer between the inlet channel and cake+cat+wall control volumes, $c_{p 1}$ is the constant pressure specific heat of the exhaust gas mixture in the inlet channel and $T_{w}$ is the absolute temperature of the cake+cat+wall control volume.

A detailed derivation of the energy balance equations in the inlet channel and outlet channel is shown in Appendix C.

Steady form of energy conservation is employed in the outlet channel also to solve for the temperature of the exhaust gas mixture as it passes through the outlet channel. Axial conduction through the exhaust gas mixture is neglected in the outlet channel since convection is the dominant mechanism of energy transfer. Assuming constant specific heat $c_{p 2}$ in the outlet channel, this relation can be expressed as:

$$
\frac{d T_{2}}{d x}=-\frac{4}{a} \frac{h_{2}}{\rho_{2} v_{2} c_{p 2}}\left(T_{2}-T_{w}\right)
$$

where $T_{2}$ is the absolute temperature of the exhaust gas mixture in the outlet channel, $h_{2}$ is the heat transfer coefficient for convective heat transfer between the outlet channel and cake+cat+wall control volumes, $c_{p 2}$ is the constant pressure specific heat of the exhaust gas mixture in the outlet channel and $T_{w}$ is the absolute temperature of the cake+cat+wall control volume. Here, an assumption that is made in Equations (3.25) and (3.26) is that the specific heat of the exhaust gas mixture is a constant, and can be taken out of the gradient ( $\frac{d}{d x}$ term). This is not true in cases where the specific heat is not constant through the axial length of the $\mathrm{CPF}$, for example, during active regeneration of the particulate filter where temperature differences as high as $100^{\circ} \mathrm{C}$ are observed. However, from looking up values of specific heat of dry air in a wide range of temperatures $\left(200-650^{\circ} \mathrm{C}\right)$ suggested that the changes in specific heat are less than $\pm 5 \%$ from the mean value. Hence, this assumption is considered valid for modeling the $\mathrm{CPF}$ that operates in this above temperature range.

The unsteady form of the energy balance equation is considered in order to solve for the temperature of the substrate wall. The energy balance of each discretized control volume of the cake+cat+wall element is considered to form the energy conservation equation. The 
differential form of the conservation equation used is:

$$
\begin{aligned}
\frac{\partial}{\partial t}\left(\rho_{p} c_{p, p} A_{p} \Delta x+\rho_{s} c_{p, s} w_{s} a \Delta x\right) T_{w} & =\dot{q}_{c o n v, 1-w}+\dot{q}_{c o n v, 2-w} \\
& +\dot{q}_{c o n d, c a k e}+\dot{q}_{c o n d, w a l l} \\
& +\dot{q}_{r x n}-\dot{q}_{a m b}
\end{aligned}
$$

where $T_{w}$ is the absolute temperature of the cake+cat+wall control volume, $c_{p w}$ is the constant pressure specific heat of the exhaust gas mixture in the cake+cat+wall control volume, $c_{p, p}$ is the specific heat of the PM cake layer, $\rho_{s}$ is the bulk density of the substrate wall and $c_{p, s}$ is the specific heat of the substrate wall. Here, $A_{p}$ is the cross-sectional area of the PM cake layer at a given axial $(x)$ interface as is shown in Figure 3.7, which can be calculated as $A_{p}=\frac{a^{*}+a_{c p}}{2} w_{p}$ where $a_{c p}$ is the width available to flow at the PM cake layer - catalyst washcoat interface, defined as $a_{c p}=a-2 w_{c a t}$. Equation (3.27) results from a balance of rate of energy accumulation in the cake+cat+wall control volume (LHS term of Equation (3.27)) to the rate of energy transferred via convection between inlet channel and substrate wall $\left(\dot{q}_{c o n v, 1-w}\right)$, the rate of energy transferred via convection between outlet channel and substrate wall $\left(\dot{q}_{c o n v, 2-w}\right)$, the rate of energy transferred between adjacent PM cake layer elements via conduction $\left(\dot{q}_{\text {cond,cake }}\right)$, the rate of energy transferred between adjacent substrate wall elements via conduction $\left(\dot{q}_{\text {cond,wall }}\right)$, the resultant energy release rate due to chemical reactions in the cake+cat+wall control volume $\left(\dot{q}_{r x n}\right)$ and the rate of energy loss to the ambient $\left(\dot{q}_{a m b}\right)$.

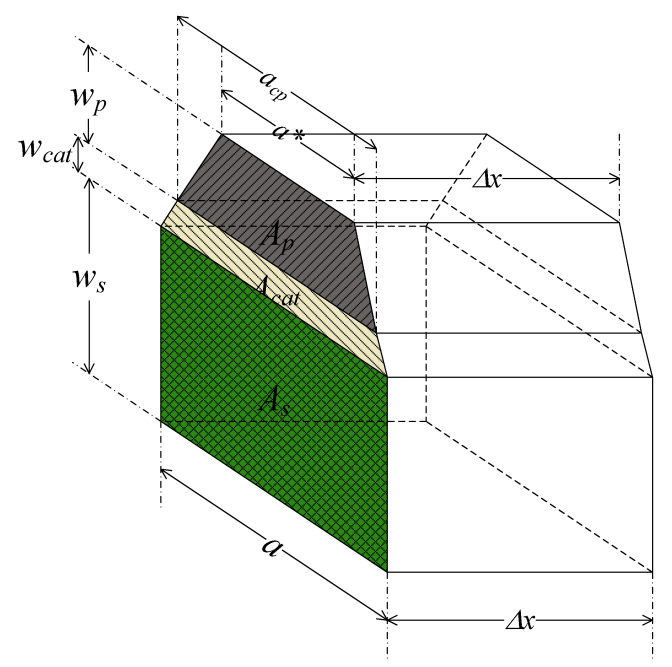

Figure 3.7: Diagram showing an isometric view of the cake+cat+wall control volume with the geometric dimensions as well as interface areas 
The RHS terms in Equation (3.27) are calculated as:

$$
\begin{aligned}
\dot{q}_{c o n v, 1-w} & =a^{*} \Delta x \rho_{w} v_{w} c_{p w} T_{w}+a^{*} \Delta x h_{1}\left(T_{1}-T_{w}\right) \\
\dot{q}_{c o n v, 2-w} & =-a \Delta x \rho_{w} v_{w} c_{p w} T_{w}+a \Delta x h_{2}\left(T_{2}-T_{w}\right) \\
\dot{q}_{c o n d, c a k e} & =\lambda_{p} \Delta x \frac{\partial\left(A_{p} \frac{\partial T_{w}}{\partial x}\right)}{\partial x} \\
\dot{q}_{c o n d, w a l l} & =\lambda_{s} \Delta x \frac{\partial^{2} T_{w}}{\partial x^{2}} a w_{s} \\
\dot{q}_{a m b} & =(h A)_{a m b}\left(T_{w}-T_{a m b}\right) \\
\dot{q}_{r x n} & =\sum_{i}\left(-\Delta H_{i} R_{i} \bar{a}_{p} w_{p} \Delta x\right) \\
& +\sum_{j}\left(-\Delta H_{j} R_{j} \bar{a}_{c a t} w_{c a t} \Delta x\right) \\
& +\sum_{k}\left(-\Delta H_{k} R_{k} a w_{s} \Delta x\right)
\end{aligned}
$$

where $\lambda_{p}$ is the thermal conductivity of the PM cake layer, $\lambda_{s}$ is the thermal conductivity of the substrate wall and $w_{s}$ is the thickness of the substrate wall, $\overline{a_{p}}$ is the midpoint width of the PM cake layer control volume $\left(\overline{a_{p}}=\frac{a_{c p}+a^{*}}{2}\right), \bar{a}_{c a t}$ is the midpoint width of the catalyst washcoat control volume $\left(\bar{a}_{c a t}=\frac{a+a_{c p}}{2}\right), \Delta H_{s}(s=i, j, k)$ are the rates of energy released by reactions $s$ and $R_{s}(s=i, j, k)$ are rates of reactions $s$. Here, the index $i$ represents all reactions taking place in the PM cake layer, $j$ represents those taking place in the catalyst washcoat and $k$ represents all those occurring in the substrate wall. Also, $h_{a m b}$ is the equivalent convectional heat transfer coefficient to the ambient and $T_{a m b}$ is the absolute ambient temperature.

Note here that the temporal change in energy content of the catalyst washcoat (in the LHS term) and the corresponding energy conduction rate through the catalyst washcoat (in the RHS) have not been considered in this model since:

- These quantities are assumed to be small compared to the corresponding quantities associated with the PM cake layer and the substrate wall and hence do not affect the overall quality of results, and

- The thermal properties of catalyst washcoat are not available at this point. The addition of this term to the governing equation and hence to the wall temperature solver can be carried out as part of future work in order to be accurate. Again, this is not anticipated to be a significant model improvement. 


\section{Numerical Solution Procedure}

In order to solve for the absolute temperature of the exhaust gas mixture as it flows through the inlet channel, cake+cat+wall and outlet channel control volumes, the energy balance equations as shown in Equations (3.25), (3.26) and (3.27) have to be solved. A detailed derivation of the energy balance equations is shown in Appendix C. These calculations are carried out by separate submodels, shown in Figure E.1 by blocks titled 'calculate inlet channel temperature field', 'calculate outlet channel temperature field' and 'calculate substrate wall temperature field' and are contained in the Fortran model source code in subroutines called 'inlet_channel_temperatures_quasisteady', 'outlet_channel_temperatures_quasisteady' and 'wall_temperatures_transient' respectively.

A fourth order Runge-Kutta method is used to calculate the inlet channel temperatures from the Equations (3.25) and (3.26). 'Clean' outlet channel width $a$ and effective inlet channel width $a^{*}$ are known from the geometry and output value of PM cake layer thickness from the previous iteration of the PM filtration submodel. Convection heat transfer coefficients $h_{1}$ and $h_{2}$ are calculated from the relation as given in Equation (3.11). Densities $\rho_{1}$ and $\rho_{2}$ are known from the density calculations according to Equations (3.1). The inlet channel and outlet channel temperature solvers are invoked after the gaseous velocity solver and hence, the gaseous velocity field (and therefore the inlet channel and outlet channel velocities $\left(v_{1}\right)$ and $\left(v_{2}\right)$ ) are known.

For the solution of the wall temperature Equation (3.27), a Crank-Nicolson scheme [68] is employed which is of second order in time. A second order spatial resolution is obtained from using a central difference method [68] for spatial gradients arising from the conduction terms. Equation (3.27) can be re-written as:

$$
\frac{\partial T_{w}}{\partial t}=R H S
$$

or according to the Crank-Nicolson scheme,

$$
\frac{\left.T_{w}\right|_{t+\Delta t}-\left.T_{w}\right|_{t}}{\Delta t}=\frac{1}{2}\left[\left.R H S\right|_{t+\Delta t}+\left.R H S\right|_{t}\right]
$$

or

$$
\left.T_{w}\right|_{t+\Delta t}=\left.T_{w}\right|_{t}+\frac{\Delta t}{2}\left[\left.R H S\right|_{t+\Delta t}+\left.R H S\right|_{t}\right]
$$

Equation (3.31) can be solved using a tri-diagonal solver [69] to obtain wall temperatures $T_{w}$. In Equation (3.31), the source terms on the right-hand side are linearized using the 
formulae:

$$
\begin{aligned}
\left.R H S\right|_{t} & =S_{C, w, 1}+\left.S_{P, w, 1} T_{w}\right|_{t} \\
\left.R H S\right|_{t+\Delta t} & =S_{C, w, 2}+\left.S_{P, w, 2} T_{w}\right|_{t+\Delta t}
\end{aligned}
$$

where $S_{C}$ stands for the constant part of the source term while $S_{P}$ is the coefficient of $T_{w}$. The condition imposed on $S_{P}$ is that it be always non-positive to avoid physically unrealistic solutions to $T_{w}$. This condition is imposed by ensuring that the total energy release rate term $\dot{q}_{r x n}$ is always checked for its sign when adding to $S_{C}$ or $S_{P}$.

$$
\begin{aligned}
& \dot{q}_{r x n,+}=\max \left(\dot{q}_{r x n}, 0\right) \\
& \dot{q}_{r x n,-}=\frac{\min \left(\dot{q}_{r x n}, 0\right)}{T_{w}}
\end{aligned}
$$

These variables maintain their signs, i.e., $\dot{q}_{r x n,+}$ is always non-negative and $\dot{q}_{r x n,-}$ is always non-positive. Now, the source term components shown in Equations (3.32) are calculated as:

$$
\begin{aligned}
S_{C, w, 1}= & \left.h_{1} T_{1} a^{*} \Delta x\right|_{t}+\left.h_{2} T_{2} a \Delta x\right|_{t}+\left.\rho_{w} v_{w} c_{p 1} T_{1} a^{*} \Delta x\right|_{t} \\
& -\left.\rho_{w} v_{w} c_{p 2} T_{2} a \Delta x\right|_{t}+\left.\dot{q}_{r x n,+}\right|_{t} \\
S_{P, w, 1}= & -\left.h_{1} a^{*} \Delta x\right|_{t}-\left.h_{2} a \Delta x\right|_{t}+\left.\dot{q}_{r x n,-}\right|_{t} \\
S_{C, w, 2}= & \left.h_{1} T_{1} a^{*} \Delta x\right|_{t+\Delta t}+\left.h_{2} T_{2} a \Delta x\right|_{t+\Delta t}+\left.\rho_{w} v_{w} c_{p 1} T_{1} a^{*} \Delta x\right|_{t+\Delta t} \\
& -\left.\rho_{w} v_{w} c_{p 2} T_{2} a \Delta x\right|_{t+\Delta t}+\left.\dot{q}_{r x n,+}\right|_{t+\Delta t} \\
S_{P, w, 2}= & -\left.h_{1} a^{*} \Delta x\right|_{t+\Delta t}-\left.h_{2} a \Delta x\right|_{t+\Delta t}+\left.\dot{q}_{r x n,-}\right|_{t+\Delta t}
\end{aligned}
$$

\subsubsection{Gaseous Species Concentrations Field - Catalyst Submodel}

\section{Equations of Conservation of Mass of Gaseous Chemical Species}

Mass conservation of individual chemical species through the cake+cat+wall control volume forms the governing equation that is solved in order to calculate concentrations of individual chemical species that the exhaust gas mixture is comprised of. Please refer to Appendix D for a detailed derivation of the conservation equations. Equation (D.3) is reproduced below in differential form as Equation (3.35) for convenience.

$$
a v_{w} \frac{d Y_{i}}{d y}-\frac{d}{d y}\left(D_{i} a \frac{d Y_{i}}{d y}\right)=-\frac{\bar{a}}{\left(\bar{\rho}_{e x h}\right)_{w}} \sum_{j} \xi_{i j} R_{j}
$$


where $Y_{i}$ is the mole fraction of chemical species $i$ in the cake+cat+wall control volume, $D_{i}$ is the effective diffusivity of chemical species $i$, the $y$ co-ordinate denotes the transverse or into-the-wall direction, $\left(\bar{\rho}_{\text {exh }}\right)_{m}$ is the molecular density of the exhaust gas mixture in the cake+cat+wall control volume (calculated from ideal gas law), $\xi_{i j}$ is the stoichiometric coefficient of species $i$ in reaction $j$ and $R_{j}$ is the rate of reaction $j$. Here, $i$ is the index of the chemical species as shown in Table 3.1. Note that since $N_{2}$ is not involved in any chemical reaction, solution for concentration of $N_{2}$ is not performed. Also, species balance of PM (carbon - index 6) is not carried out through solution of Equation (3.35) but through a PM oxidation submodel as explained later in section 3.4.6.

The major assumptions associated with the derivation of governing equation (3.35) are:

1. Molecular density of the exhaust gas mixture $\left(\bar{\rho}_{\text {exh }}\right)_{w}$ is constant throughout the cake+cat+wall control volume. This is in order to be consistent with the assumption that the density of exhaust gas mixture is constant in the cake+cat+wall volume (equation 3.1), and it presents the advantage of further simplification of the governing equation.

2. Concentrations of individual chemical species at the boundary $B_{1}$, i.e., the inlet channel - PM cake layer interface, of all axially discretized cake+cat+wall control volumes are assumed to be known and equal to the CPF inlet concentration of that chemical species at the given point in time, which is a model input.

3. Concentration of individual chemical species in the outlet channel is assumed to be equal to the concentration at the boundary $B_{2}$, i.e., the substrate wall - outlet channel interface.

Table 3.1: Tabulated list of chemical species considered in the CPF model version 4.0

\begin{tabular}{|l|l|l|}
\hline Index & Chemical Species & Molecular Formula \\
\hline 1 & n-Dodecene $(\mathrm{g})$ & $\mathrm{C}_{12} \mathrm{H}_{24}$ \\
2 & Oxygen $(\mathrm{g})$ & $\mathrm{O}_{2}$ \\
3 & Nitrogen $(\mathrm{g})$ & $\mathrm{N}_{2}$ \\
4 & Carbon dioxide $(\mathrm{g})$ & $\mathrm{CO}_{2}$ \\
5 & Water $(\mathrm{g})$ & $\mathrm{H}_{2} \mathrm{O}$ \\
6 & Carbon $(\mathrm{s})$ & $\mathrm{C}$ \\
7 & Carbon monoxide $(\mathrm{g})$ & $\mathrm{CO}$ \\
8 & Nitric oxide $(\mathrm{g})$ & $\mathrm{NO}$ \\
9 & Nitrogen dioxide $(\mathrm{g})$ & $\mathrm{NO}_{2}$ \\
\hline
\end{tabular}


The boundary conditions used in solving equation (3.35) are:

$$
\begin{aligned}
\left.Y_{i}\right|_{B_{1}} & =Y_{1, i} \\
\left.\frac{d Y_{i}}{d y}\right|_{B_{2}} & =0
\end{aligned}
$$

where $Y_{2, i}$ is the mole fraction of chemical species $i$ in the outlet channel control volume and $B_{2}$ denotes the boundary that is at the interface of the cake+cat+wall and outlet channel control volumes (see Figure 3.9).

\section{Numerical Solution Procedure}

The catalyst submodel in the CPF model keeps track of gaseous species as the exhaust gas mixture passes through the inlet channel, PM cake layer, catalyst washcoat (if present), substrate wall and outlet channel. During discussions with industry partners as part of the development effort, it was learned that there are different types of catalyzed particulate filters that are in use currently that differ in the location of catalyst washcoat in relation to the substrate wall. In order to be able to simulate various catalyst configurations, it was decided to construct a general framework for modeling the catalyst in a CPF and specific cases simulated by varying input parameters to the catalyst submodel while simulating different experiments. Figure 3.8 gives a schematic representation of two different types of catalyst location that are found in catalyzed particulate filters by different vendors/manufacturers ( $\mathrm{a}$ and $\mathrm{b}$ ), the common framework in the catalyst submodel (c) and domain setup for the two types of catalyst used in the model ( $d$ and e respectively).

In Figure 3.8, for the first type of catalyst (sub-figure a.) or "pore type", the catalyst is entirely present on the inner curved surface area of the pores in the substrate wall and not on the surface of the wall at all. In the second type (b.) or "surface type", the catalyst is located on the inlet-channel-side surface of the substrate wall as a separate sub-layer (of 10-20 $\mu \mathrm{m}$ thickness) and does not penetrate into the pores in the substrate wall. The common model framework (c.) contains the thickness of the catalyst washcoat $\left(w_{c a t}\right)$ and penetration depth of catalyst washcoat into the substrate wall thickness $\left(d_{\text {pen }}\right)$ as two input parameters, which for simulating pore type catalyst can be specified as: $w_{c a t}=0$ and $d_{\text {pen }}=w_{s}$ where $w_{s}$ is the thickness of the substrate wall and for surface type catalyst: $w_{\text {cat }}=20 \mu \mathrm{m}$ and $d_{\text {pen }}=0$. The results are as shown in sub-figures d. and e. respectively.

Appendix D gives a detailed derivation of the equations used for solving the gaseous species mole fractions field in the particulate filter model. The chemical species that are considered in the CPF model version 4.0 are given in Table 3.1. Figure 3.9 shows a schematic representation of the chemical reactions that take place in the different locations (domains) of the cake+cat+wall control volume involving these chemical species. The 
a.

Pore Type b.

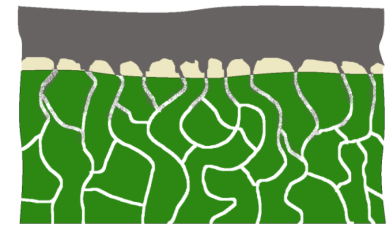

Surface Type

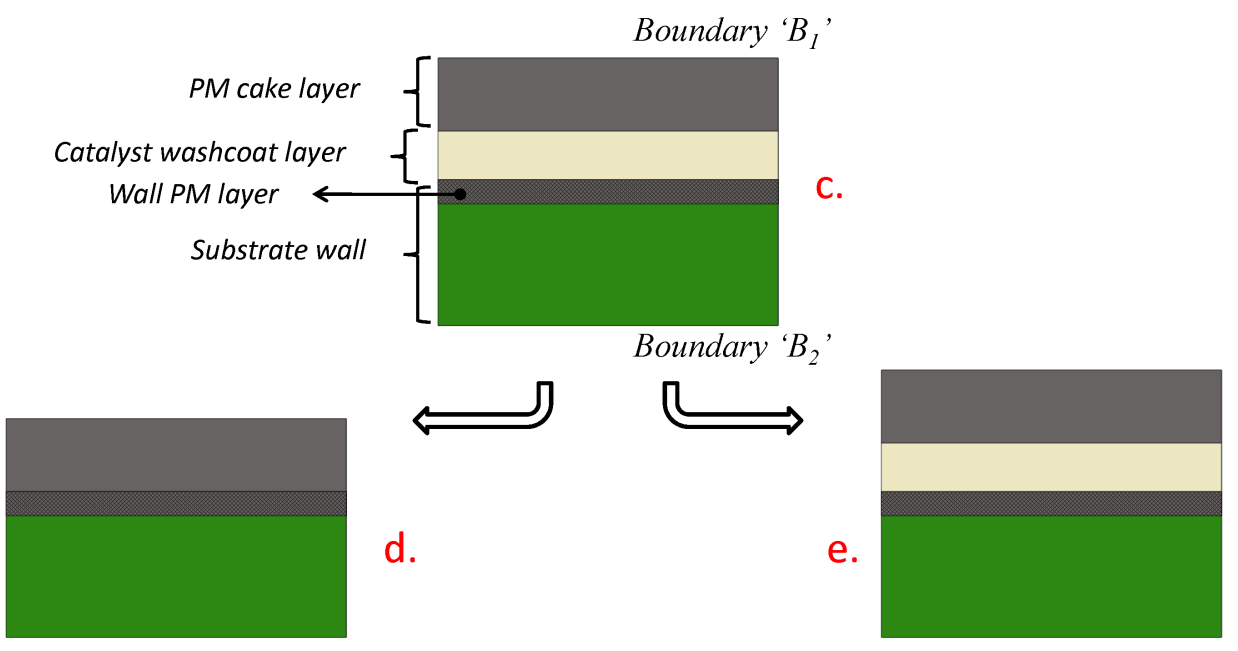

Figure 3.8: Schematic representation of two different types of catalyst location (a. and b.), model framework (c.) and model setup for both catalyst location types when simulating (d. and e.)

Table 3.2: Chemical reactions considered in the CPF model. Here, $i=$ cake, wall

\begin{tabular}{|c|c|c|c|}
\hline $\mathrm{C}_{12} \mathrm{H}_{24}+(18) \mathrm{O}_{2}$ & $\rightarrow$ & $(12) \mathrm{CO}_{2}+(12) \mathrm{H}_{2} \mathrm{O}$ & $\left(R_{H C}\right)$ \\
\hline $\mathrm{CO}+(12) \mathrm{O}_{2}$ & $\rightarrow$ & $\mathrm{CO}_{2}$ & $\left(R_{C O}\right)$ \\
\hline $\mathrm{NO}+\left(\frac{1}{2}\right) \mathrm{O}_{2}$ & $\leftrightarrow$ & $\mathrm{NO}_{2}$ & $\left(R_{N O}\right)$ \\
\hline$C+\left(1-\frac{f_{C O}}{2}\right) O_{2}$ & $\rightarrow$ & $\left(f_{C O}\right) C O+\left(1-f_{C O}\right) C_{2}$ & $\left(R_{t h, i}\right)$ \\
\hline $\mathrm{C}+\left(2-g_{\mathrm{CO}}\right) \mathrm{NO}_{2}$ & $\rightarrow$ & $\left(g_{C O}\right) \mathrm{CO}+\left(1-g_{C O}\right) \mathrm{CO}_{2}+\left(2-g_{C O}\right) \mathrm{NO}$ & $\left(R_{\mathrm{NO}_{2}, i}\right)$ \\
\hline
\end{tabular}

stoichiometric chemical reactions corresponding to the reaction numbers $\left(R_{1}, R_{2}\right.$, etc.) are given in Table 3.2. 


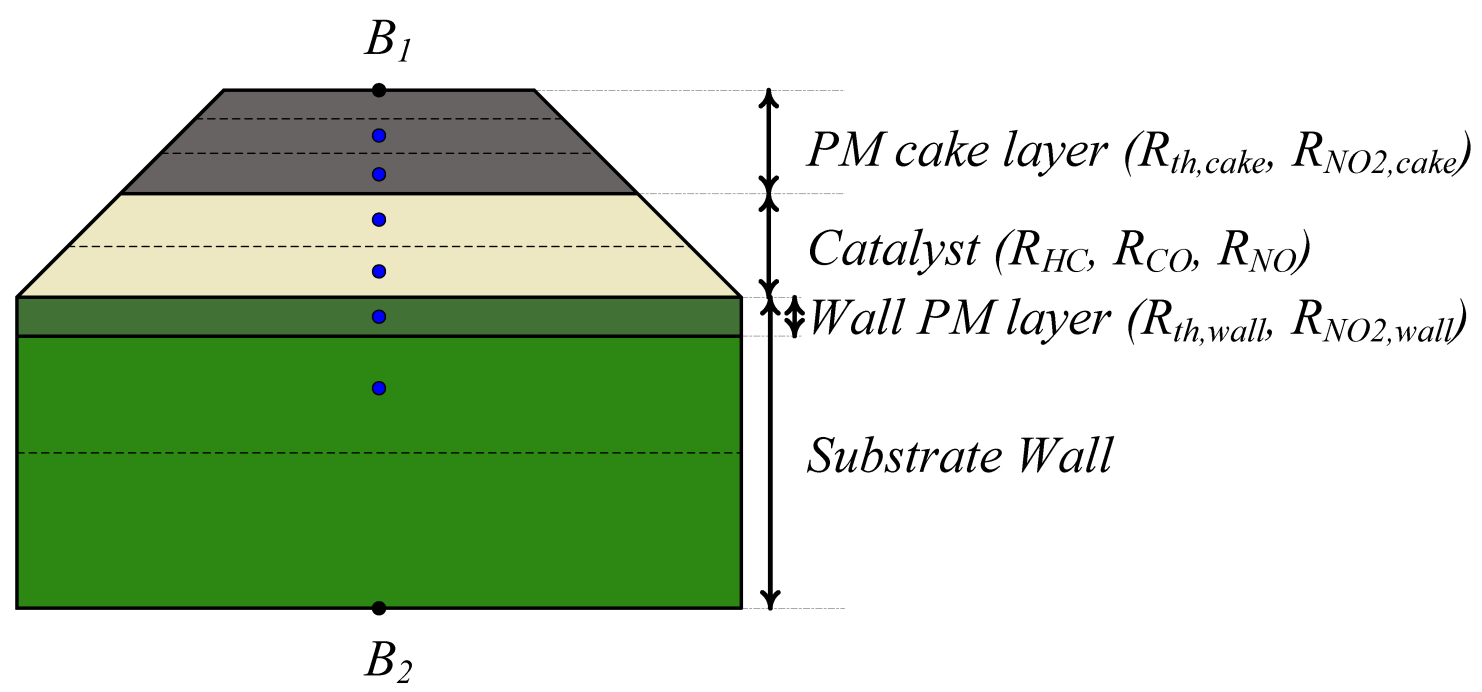

Figure 3.9: Schematic representation of cake+cat+wall control volume, showing the different domains and the chemical reactions associated with them - reactions are as given in Table 3.2

The catalyst submodel uses the governing equation (Equation (D.3)) that includes mass change rates of each individual chemical species due to convection, diffusion and reaction occurring in each discretized control volume in the PM cake layer, catalyst washcoat and substrate wall.

The rates of the chemical reactions involving $\mathrm{HC}$ oxidation, $\mathrm{CO}$ oxidation and $\mathrm{NO}$ oxidation shown in Table 3.2 that are used in Equation (3.35) are expressed as:

$$
\begin{aligned}
R_{H C} & =\frac{A_{H C}\left(T_{w}\right)^{x_{H C}} e^{-\frac{E a_{H C}}{R T_{w}}}\left[C_{12} H_{24}\right]\left[O_{2}\right]}{G_{1}} \\
R_{C O} & =\frac{A_{C O}\left(T_{w}\right)^{x_{C O}} e^{-\frac{E a_{C O}}{R T_{w}}}[C O]\left[O_{2}\right]}{G_{2}} \\
R_{N O} & =\frac{A_{N O}\left(T_{w}\right)^{x_{N O}} e^{-\frac{E a_{N O}}{R T_{w}}}}{G_{3}}\left[[N O]\left[O_{2}\right]^{1 / 2}-\frac{\left[N O_{2}\right]}{K_{C}}\right]
\end{aligned}
$$

where $A_{H C}$ is the pre-exponential factor of the $\mathrm{HC}$ oxidation reaction, $x_{H C}$ is the temperature order of dependence of the $\mathrm{HC}$ oxidation reaction, $E a_{H C}$ is the activation energy of the $\mathrm{HC}$ oxidation reaction, $\left[\mathrm{C}_{12} \mathrm{H}_{24}\right]$ is the concentration of $\mathrm{C}_{12} \mathrm{H}_{24},\left[\mathrm{O}_{2}\right]$ is the concentration of $\mathrm{O}_{2}, A_{C O}$ is the pre-exponential factor of the $\mathrm{CO}$ oxidation reaction, $x_{C O}$ is the temperature order of the $\mathrm{CO}$ oxidation reaction, $E a_{C O}$ is the activation energy of 
the $\mathrm{CO}$ oxidation reaction, $[\mathrm{CO}]$ is the concentration of $C O, A_{N O}$ is the pre-exponential factor of the NO oxidation reaction, $x_{N O}$ is the temperature order of dependence of the NO oxidation reaction, $E a_{N O}$ is the activation energy of the NO oxidation reaction, $[N O]$ is the concentration of $\mathrm{NO}$ and $\left[\mathrm{NO}_{2}\right]$ is the concentration of $\mathrm{NO}_{2} . \mathrm{K}_{\mathrm{C}}$ is the equilibrium constant based on concentrations which is in turn obtained from the equilibrium constant based on partial pressures according to the relation:

$$
K_{C}=K_{P} \sqrt{\frac{R T_{w}}{P}}
$$

and $K_{P}$ is the equilibrium constant based on partial pressures calculated for a pure mixture of $\mathrm{NO}, \mathrm{O}_{2}$ and $\mathrm{NO}_{2}$ as:

$$
K_{P}=e^{\left(\frac{6950.09}{T_{w}}-9.12\right)}
$$

The inhibition factors $G_{1}$ through $G_{3}$ appearing in equations (3.37) are given as:

$$
\begin{aligned}
& G_{1}=\left(\frac{T}{T_{r e f}}\right)^{x_{H C}^{G}}\left(1+K_{a, 1} C_{C O}+K_{a, 2} C_{C_{12} H_{24}}\right)^{2} \\
& \left(1+K_{a, 3} C_{C O}^{2} C_{C_{12} H_{24}}^{2}\right) \\
& \left(1+K_{a, 4} C_{N O}^{0.7}\right) \\
& G_{2}=\left(\frac{T}{T_{r e f}}\right)^{x_{C O}^{G}}\left(1+K_{a, 5} C_{C O}+K_{a, 6} C_{C_{12} H_{24}}\right)^{2} \\
& \left(1+K_{a, 7} C_{C O}^{2} C_{C_{12} H_{24}}^{2}\right) \\
& \left(1+K_{a, 8} C_{N O}^{0.7}\right) \\
& G_{3}=\left(\frac{T}{T_{r e f}}\right)^{x_{N O}^{G}}\left(1+K_{a, 9} C_{C O}+K_{a, 10} C_{C_{12} H_{24}}\right)^{2} \\
& \left(1+K_{a, 11} C_{C O}^{2} C_{C_{12} H_{24}}^{2}\right) \\
& \left(1+K_{a, 12} C_{N O}^{0.7}\right)
\end{aligned}
$$

where the adsorption factors $K_{a, i}$ are expressed as functions of adsorption pre-exponential factors $K_{a 0, i}$ and adsorption heats $\Delta H_{a, i}$ as:

$$
K_{a, i}=K_{a 0, i} e^{\frac{\Delta H_{a, i}}{\bar{R} T}}
$$


Equation (3.35) can be discretized to form a tri-diagonal system of equations, which can then be solved using a tri-diagonal solver [69] and is solved with the following boundary conditions:

$$
\begin{aligned}
\left.Y_{i}\right|_{B_{1}} & =Y_{i, i n} \\
\left.\frac{d Y_{i}}{d y}\right|_{B_{2}} & =0
\end{aligned}
$$

The outlet channel mole fractions of each chemical species at each axial location are then set equal to the cake+cat+wall mole fractions at the boundary $B_{2}$.

$$
Y_{2, i}=\left.Y_{i}\right|_{B_{2}}
$$

\subsubsection{PM Mass Balance and PM Oxidation}

In the $\mathrm{CPF}$ model, $\mathrm{PM}$ is considered as being retained and oxidized in the PM cake layer as well as substrate wall of the single channel model. PM in the PM cake layer is numerically sub-divided into a user-specified number of transverse discretizations ( $n$ div $v_{\text {cake }}$ as shown in Figure D.2). All PM that is collected in the different 'slabs' in the substrate wall is considered to be accumulated in a separate layer called wall PM layer as shown in Figure 3.9. This simplifies the calculation of the oxidation rate of wall PM as a single value rather than discretized values throughout the substrate wall thickness. Also, the lack of knowledge about the exact location of PM in the substrate wall further necessitates this approach which is similar to previous research [64].

\section{PM Mass Balance}

In the CPF model, the conservation of PM mass can be stated mathematically as:

$$
\dot{m}_{i n}=\dot{m}_{r e t}+\dot{m}_{o x}+\dot{m}_{\text {out }}
$$

The flow rate of PM into the $\mathrm{CPF}$ at any given instant is given as:

$$
\dot{m}_{i n}=C_{i n} Q_{s t d}
$$


where $C_{i n}$ is the CPF inlet PM concentration at STP (expressed in $\frac{k g}{s t d . m^{3}}$ ) and $Q_{s t d}$ is the standard volumetric flow rate of exhaust gas into the CPF. This is the total PM inlet rate. Hence, PM inlet rate into each inlet channel of the CPF is given as:

$$
\dot{m}_{i n, c h}=\frac{C_{i n} Q_{s t d}}{n_{\text {cells }}}
$$

where $n_{\text {cells }}$ is the number of inlet channels. In the case of the PM cake layer or the substrate wall if the PM cake layer has not formed yet, the flow rate of PM into each axial discretization of the CPF model is weighted according to the equation:

$$
\dot{m}_{i n, a x i a l}=\dot{m}_{i n, c h} \frac{v_{w}(x)}{\bar{v}_{w}}
$$

where $v_{w}(x)$ is the local wall flow velocity at location $(x)$ and $\bar{v}_{w}$ is the average wall-flow velocity.

\section{Cake PM oxidation}

Rate of PM oxidation in the PM cake layer due to thermal and passive PM oxidation reactions is calculated by the relation:

$$
\dot{m}_{o x, c a k e}=m_{\text {cake }}\left[(-1) R_{t h, c a k e}+(-1) R_{N_{2}, \text { cake }}\right]
$$

where $m_{\text {cake }}$ is the mass of PM in the PM cake layer at any given instant, $R_{\text {th,cake }}$ is the rate of thermal PM oxidation reaction in the PM cake layer and $R_{\mathrm{NO}_{2} \text {,cake }}$ is the rate of $\mathrm{NO}_{2}$-assisted PM oxidation reaction in the PM cake layer.

In Equation (3.48), the rates of thermal and passive PM oxidation reactions in PM cake layer are calculated by:

$$
\begin{aligned}
R_{\text {th }, \text { cake }} & =S_{p} A_{\text {th,cake }}\left[Y_{\mathrm{O}_{2}}\right]\left(T_{w}\right)^{x_{\text {th,cake }}} e^{-\frac{E a_{\text {th }, \text { cake }}}{R T_{w}}} \\
R_{\mathrm{NO}_{2}, \text { cake }} & =S_{p} A_{\mathrm{NO}_{2}, \text { cake }}\left[Y_{\mathrm{NO}_{2}}\right]\left(T_{w}\right)^{x_{N O_{2}, \text { cake }}} e^{-\frac{E a_{N O_{2}, \text { cake }}}{R T_{w}}}
\end{aligned}
$$

where $S_{p}$ is the specific area of PM $\left(=\rho_{p} A_{p}\right), A_{p}$ is the area of PM per unit mass of PM $\left(=1 x 10^{5}\left[\frac{m^{2}}{k g}\right]\right), A_{t h, c a k e}$ is the thermal pre-exponential factor in PM cake layer, $x_{t h, c a k e}$ is the thermal temperature order of dependence in PM cake layer, $E a_{t h, c a k e}$ is the thermal

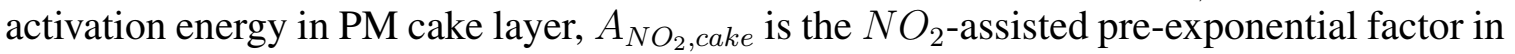
PM cake layer, $x_{\mathrm{NO}_{2} \text {,cake }}$ is the $\mathrm{NO}_{2}$-assisted temperature order of dependence in PM cake layer and $\mathrm{Ea}_{\mathrm{NO}_{2} \text {,cake }}$ is the $\mathrm{NO}_{2}$-assisted activation energy in PM cake layer. 


\section{Wall PM Oxidation}

As PM flows into the particulate filter with the exhaust gas mixture and as it flows through the porous substrate wall of the filter, it gets collected in the pores of the wall. The rate of this addition of PM is determined by the filtration efficiency of the substrate wall as calculated in the filtration submodel of the CPF model (section 3.4.7). Initially, a clean wall is assumed. Then, as the PM mass accumulates in the various sub-layers of the substrate wall according to the local filtration efficiency calculated, wall PM mass increases. The mass of PM in the wall is the sum of mass retained in all the slabs of the wall discretized in the axial $(x)$ direction as well as transverse $(y)$ direction as shown in Figure 3.10. For

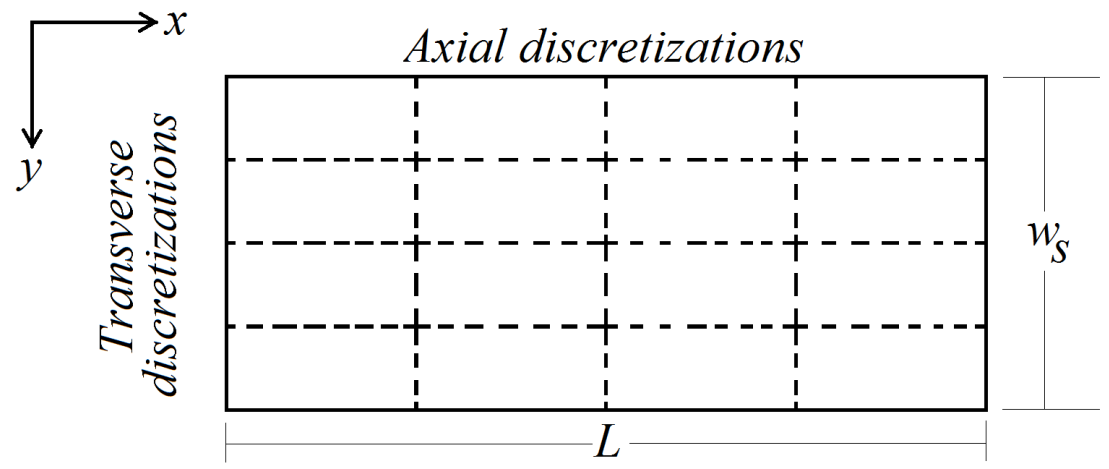

Figure 3.10: Numerical discretizations in the axial $(x)$ and transverse $(y)$ directions in the substrate wall sub-domain of the CPF model

modeling the oxidation of this PM, the concept of a virtual wall layer is used. For this simplification, all PM mass in the substrate wall is assumed to be in a "virtual layer" whose thickness is calculated as if it were deposited at the same density as the PM cake layer. Mathematically,

$$
w_{\text {wall }}(x)=\frac{m_{\text {wall }}(x)}{\left.\rho_{p} a \Delta x\right)}
$$

where $m_{\text {wall }}(x)$ is the PM mass accumulated in one axial wall element $\left(m_{\text {wall }}(x)=\int_{i=1}^{n_{\text {slab }}} m_{\text {slab }}(x, y)\right), \rho_{p}$ is the packing density of the PM cake layer, $a$ is the clean channel width, and $\Delta x=\frac{L}{n_{x d i v}}$ where $n_{x d i v}$ is the number of discretizations in the axial $(x)$ direction. In the catalyst sub-model, an equal sub-division of all sub-domains in the transverse direction is assumed according to the user-specified number of sub-divisions in the cake, catalyst and wall. The first node (top of PM cake layer) is at the inlet-channel/PM cake layer boundary and the last node (bottom of substrate wall) is at the substrate wall/outlet channel boundary. Then, in order for the catalyst sub-model to be consistent with the virtual wall PM layer thickness, the location of the first node of the substrate wall within the substrate wall is set to half this thickness $\left(w_{\text {wall }}\right)$. Figure 3.11 shows the addition of the virtual wall layer in the calculation of the domain grid in the catalyst sub-model. The virtual wall layer thus becomes the first "slab" of the substrate 
wall, having a thickness $\left(w_{\text {wall }}\right)$ different from the thickness of the other "slabs" which is calculated as: $\Delta y^{*}=\frac{w_{s}-w_{\text {wall }}}{n_{\text {slab }}-1}$ where $n_{\text {slab }}$ is the user-specified number of discretizations of the wall sub-domain in the transverse $(y)$ direction.

(a)

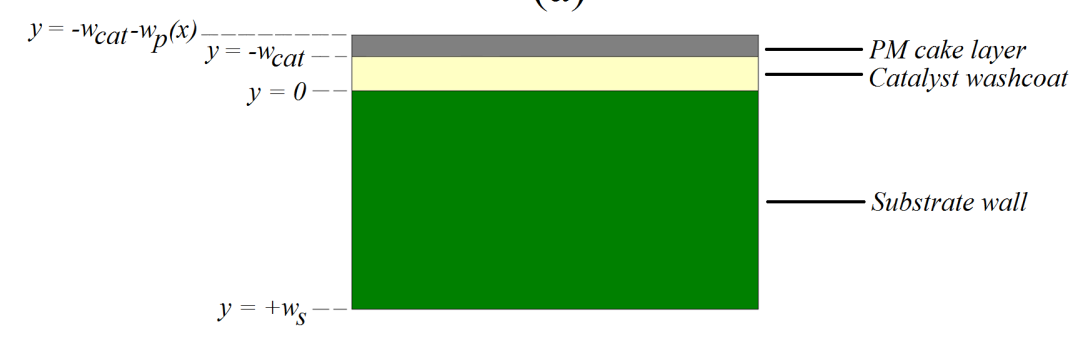

(b)

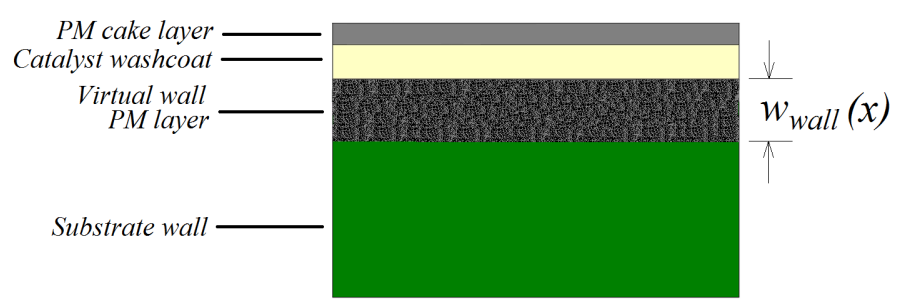

Figure 3.11: Schematic showing catalyst sub-model domain grid: (a) before the addition of the virtual wall PM layer, and (b) after the addition of the virtual wall PM layer

Wall PM oxidation rate is then calculated as:

$$
\dot{m}_{\text {ox,wall }}=m_{\text {wall }}\left[(-1) R_{\text {th,wall }}+(-1) R_{\mathrm{NO}_{2}, \text { wall }}\right]
$$

where $R_{\text {th,wall }}$ and $R_{\mathrm{NO}_{2}, \text { wall }}$ are calculated by:

$$
\begin{aligned}
R_{\text {th,wall }} & =S_{p} A_{t h, \text { wall }}\left[Y_{\mathrm{O}_{2}}\right]\left(T_{w}\right)^{x_{t h, \text { wall }}} e^{-\frac{E a_{\text {th }, \text { wall }}}{R T_{w}}} \\
R_{\mathrm{NO}_{2}, \text { wall }} & =S_{p} A_{\mathrm{NO}_{2}, \text { wall }}\left[Y_{\mathrm{NO}_{2}}\right]\left(T_{w}\right)^{x_{N O_{2}, \text { wall }}} e^{-\frac{E a_{N O_{2}, \text { wall }}}{R T_{w}}}
\end{aligned}
$$

where $S_{p}$ is the specific area of PM $\left(=\rho_{p} A_{p}\right), A_{p}$ is the area of PM per unit mass of $\mathrm{PM}\left(=1 x 10^{5}\left[\frac{m^{2}}{\mathrm{~kg}}\right]\right), A_{t h, \text { wall }}$ is the thermal pre-exponential factor in wall, $x_{t h, \text { wall }}$ is the thermal temperature order of dependence in wall, $E a_{t h, w a l l}$ is the thermal activation energy in wall, $\mathrm{ANO}_{2}$,wall is the $\mathrm{NO}_{2}$-assisted pre-exponential factor in wall, $x_{\mathrm{NO}_{2} \text {,wall }}$ is the passive temperature order of dependence in wall and $E a_{N O_{2} \text {,wall }}$ is the passive activation energy in wall. Here, $Y_{\mathrm{O}_{2}}$ and $Y_{\mathrm{NO}_{2}}$ are the mole fractions of oxygen and nitrogen dioxide at the first node of the wall sub-domain of the cake+cat+wall domain. 


\subsubsection{Filtration of PM by PM Cake Layer and Substrate Wall}

In the CPF model, filtration of particulate matter occurs in two locations: PM cake layer and substrate wall. These are considered to be sequentially filtering PM at all axial locations in the CPF single channel model. Figure 3.12 shows a graphical representation of the wall and PM cake layer filters and calculation of PM mass captured in each filter as functions of their filtration efficiencies.

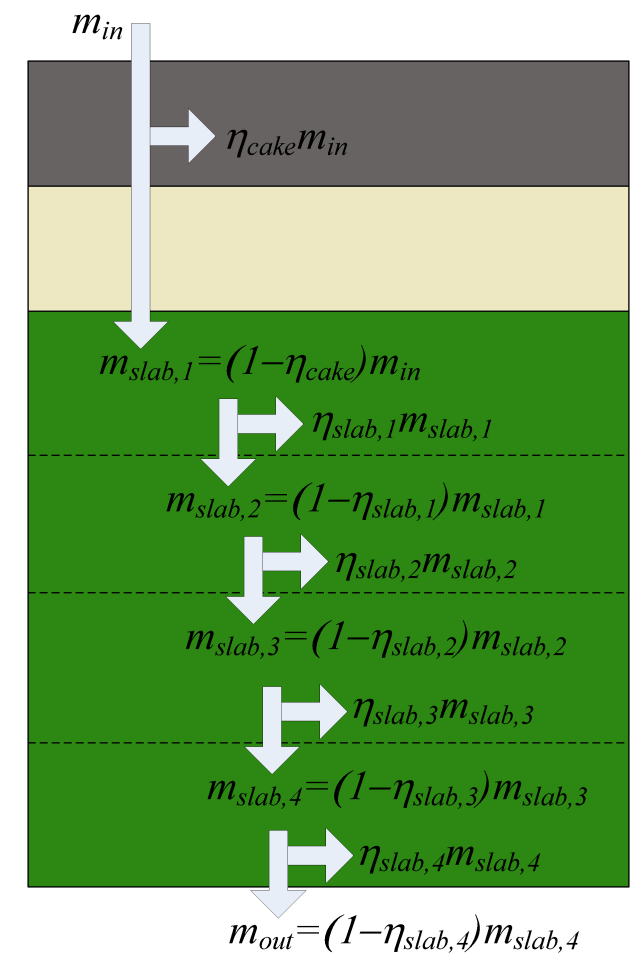

Figure 3.12: Schematic of filtration efficiency and outlet mass calculations

The overall filtration efficiency of the CPF is thus a function of PM cake and wall filtration efficiencies and can be expressed mathematically as:

$$
\eta_{\text {total }}=1-\left[\left(1-\eta_{\text {cake }}\right) \prod_{i=1}^{n_{\text {slab }}}\left(1-\eta_{\text {wall }, i}\right)\right]
$$

where $\eta_{\text {cake }}$ is the filtration efficiency of the PM cake layer and $\eta_{\text {wall }, i}$ is the filtration efficiency of the $i^{\text {th }}$ slab of the substrate wall. 
These are calculated as: [12]

$$
\eta_{\text {wall }, i}=1-e^{\left(-\frac{3 \eta_{\text {coll } l, \text { wall }}\left(1-\epsilon_{s}\right) \Delta y}{2 \epsilon_{s} d_{c, \text { wall }}}\right)}
$$

where $\eta_{\text {coll }, \text { wall }}$ is the filtration efficiency of the wall collector, $\epsilon_{s}$ is the porosity of the wall slab, $\Delta y$ is the thickness of the wall slab $\left(\Delta y=\frac{w_{s}}{n_{s l a b}}\right)$ and $d_{c, \text { wall }}$ is the diameter of the unit collector in the wall slab.

At 'clean' state, no particulate matter is present in the PM cake layer or the substrate wall. Hence, the only filter present at this point is the clean substrate wall. In the case of cordierite filters, it has been observed that the clean substrate wall with a mean pore size range of $10-20 \mu \mathrm{m}$ and porosity $\approx 0.5$ has a filtration efficiency of $70-80 \%$. This causes the PM to be deposited in the pores of the substrate wall. Modeling the filtration efficiency of the substrate wall has been carried out in accordance with existing wall filtration models that have been published in references [11] and [13]. This model is based on packed bed filtration theory [70]. The model assumes that the porous substrate wall volume can be represented by a collection of spherical unit cells, each having the same diameter $b$ and containing a unit collector of clean diameter $d_{c 0}$. The unit collector, as it collects particulate matter around it, increases uniformly in size (radially). Figure 3.13 shows a diagram of one such unit cell with the dimensions involved.

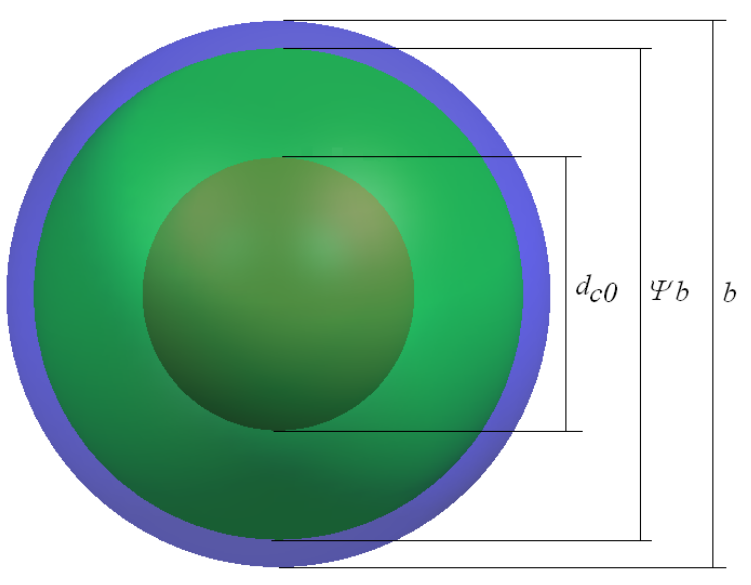

Figure 3.13: Diagram of unit cell in substrate wall

Here, the dimension $\Psi b$ is the maximum diameter to which the substrate wall is capable of capturing particulate matter, beyond which the unit cell remains constant in size regardless of the PM mass added to (captured by) the cell. $\Psi$ is called the 'percolation factor' which acts as a limiting factor on the growth of the unit collector diameter.

Clean collector diameter $d_{c 0}$ is related to the unit cell diameter $b$ via the clean porosity 
$\epsilon_{0}$ expressed as[11,12]:

$$
\frac{d_{c 0}^{3}}{b^{3}}=1-\epsilon_{0}
$$

Clean collector diameter is also related to the mean pore size $d_{\text {pore }}$ of the substrate wall and clean porosity as [12]:

$$
d_{c 0}=\frac{3}{2}\left(\frac{1-\epsilon_{0}}{\epsilon_{0}}\right) d_{\text {pore }}
$$

Effective particulate collection efficiency of the unit collector is calculated by assuming the following:

1. Particles that are in the $<10 \mathrm{~nm}$ diameter range are collected due to Brownian diffusion of the particles to the unit collector surface,

2. Interception is the dominant collection mechanism for particles of larger diameters $(10 \mathrm{~nm}-1 \mu \mathrm{m})$,

3. Inertial impaction is dominant for particles of much larger diameters $(>2 \mu m)$, and hence is neglected,

4. Gravitational settling is not important since there is continuous flow through the filter, and

5. Effective filtration efficiency of the unit cell is calculated as the equivalent of both diffusion and interception efficiencies regardless of the sizes of the individual particle that is captured.

The collection efficiency of a unit collector due to Brownian diffusion mechanism $\eta_{D}$ is given as [12]:

$$
\eta_{D}=3.5\left(\frac{\epsilon}{K}\right)^{1 / 3} P e^{-2 / 3}
$$

where $\epsilon$ is the instantaneous porosity of the wall collector, $P e$ is the Peclet number and $K$ is the Kuwabara's hydrodynamic factor, given as [12]:

$$
K=1-\frac{9}{5}(\alpha)^{1 / 3}+\alpha-\frac{1}{5} \alpha^{2}
$$

The efficiency of a single unit collector due to interception $\eta_{R}$ is expressed mathematically as[12]:

$$
\eta_{R}=\frac{3}{2}\left(\frac{\epsilon}{K}\right) \frac{N_{R}}{1+N_{R}^{\frac{3-2 \epsilon}{3 \epsilon}}}
$$

where $N_{R}$ is the particle interception parameter $\left(=\frac{d_{p}}{d_{c}}\right)$. The equivalent collection efficiency of a single wall collector due to diffusion and direct interception is then given 
as[11,12]:

$$
\eta_{D R}=\eta_{D}+\eta_{R}-\eta_{D} \eta_{R}
$$

The collection efficiency of the $i^{t h}$ wall slab $\eta_{s l a b, i}$ of thickness $\Delta y_{i}$ can then be computed as[11]:

$$
\eta_{s l a b, i}=1-e^{\left(\frac{3}{2}\left(\frac{1-\epsilon}{\epsilon}\right) \eta_{D R} \frac{\Delta y_{i}}{d_{c}}\right)}
$$

Knowing the cumulative mass of PM deposited in a unit collector $\left(m_{s}(t)\right)$ and the packing density of PM around the unit collector in the wall $\left(\rho_{p w}\right)$, the instantaneous diameter of the collector $d_{c}$ can be calculated as[11]:

$$
d_{c}=2\left[\left(\frac{d_{c 0}}{2}\right)^{3}+\frac{3}{4 \pi} \frac{m_{s}(t)}{\rho_{p w}}\right]^{\frac{1}{3}}
$$

The evolution of instantaneous porosity $\epsilon_{s}$ and permeability $k_{s}$ of the wall can then be computed as[11]:

$$
\begin{aligned}
& \epsilon_{s}=1-\left[\left(\frac{d_{c}}{d_{c 0}}\right)^{3}\left(1-\epsilon_{0}\right)\right] \\
& k_{s}=k_{0}\left(\frac{d_{c}}{d_{c 0}}\right)^{2} \frac{K(\epsilon)}{K\left(\epsilon_{0}\right)}\left(\frac{1-\epsilon_{0}}{1-\epsilon}\right)
\end{aligned}
$$

The formation of PM cake layer takes place after the substrate wall collects PM to the point where bridging of pores with PM takes place. After the formation of the PM cake layer occurs, majority of filtration takes place by the PM cake layer itself. The reason for this is that the average size of particles that are captured and retained in the PM cake layer is similar to the average size of the particulate matter coming into the CPF and hence reaching the PM cake layer. A recent research effort [32] models this evolution of filtration efficiency of a DPF by the use of a transition permeability. The concept was adopted in this thesis work to simulate the transition of filtration from deep-bed to filtration by PM cake. In the CPF model, the filtration efficiency of the PM cake layer is modeled as a function of PM cake layer thickness depending on the instantaneous permeability of the substrate wall $\left(k_{\text {wall }}\right)$ and the user-defined value of transition permeability $\left(k_{\text {trans }}\right)$ and can be expressed as:

$$
\eta_{\text {cake }}= \begin{cases}0, & \text { if } k_{\text {wall }}>k_{\text {trans }} \& w_{p}=0 \\ \phi, & \text { if } k_{\text {wall }} \leq k_{\text {trans }} \& w_{p}=0 \\ \eta_{\text {cake }, \text { Loaded }}, & \text { if } k_{\text {wall }} \leq k_{\text {trans }} \& w_{p} \neq 0\end{cases}
$$

where $w_{p}$ is the local PM cake layer thickness and $\phi$ is the partition coefficient [13]. 
In the presence of the PM cake, the cake filtration efficiency is calculated as:

$$
\eta_{\text {cake }, \text { Loaded }}=A_{\eta}\left(1-e^{\left(-\frac{3 \eta_{\text {coll }, \text { cake }}\left(1-\epsilon_{p}\right) w_{p}}{2 \epsilon_{p} d_{c, \text { cake }}}\right)}\right)
$$

where $\eta_{\text {coll,cake }}$ is the PM cake layer collector efficiency, $\epsilon_{p}$ is the PM cake layer porosity and $d_{c, \text { cake }}$ is the collector diameter in the PM cake layer (assumed to be equal to $100 \mathrm{~nm}$ ).

\section{Absolute Pressure Field and CPF Pressure Drop Calculations}

The total pressure drop across the particulate filter at any point in the simulation is the resultant of three components: pressure drop due to PM cake layer, pressure drop due to substrate wall and pressure drop due to frictional losses in the inlet channel and outlet channel. Mathematically,

$$
\Delta P_{\text {total }}=\Delta P_{\text {cake }}+\Delta P_{\text {wall }}+\Delta P_{\text {channels }}
$$

where $\Delta P_{\text {cake }}$ is the pressure drop across the PM cake layer, $\Delta P_{\text {wall }}$ is the pressure drop across the substrate wall and $\Delta P_{\text {channels }}$ is the pressure drop due to frictional losses in the inlet channel and outlet channel. The total pressure drop across the CPF is calculated from D' Arcy's law [71] which is derived from the law of conservation of momentum across the thickness of the filter medium. D'Arcy's law gives the individual pressure drop across the PM cake layer and substrate wall as:

$$
\begin{aligned}
\Delta P_{\text {cake }} & =\mu_{w} v_{w} \frac{w_{p}}{k_{p}} \\
\Delta P_{\text {wall }} & =\mu_{w} v_{w} \frac{w_{s}}{k_{s}}
\end{aligned}
$$

where $\mu_{w}$ is the dynamic viscosity of the exhaust gas mixture in the cake+cat+wall control volume, $v_{w}$ is the velocity of the exhaust gas mixture in the cake+cat+wall control volume, $w_{p}$ is the thickness of the PM cake layer, $k_{p}$ is the instantaneous permeability of the PM cake layer, $w_{s}$ is the thickness of the substrate wall and $k_{s}$ is the permeability of the substrate wall. The pressure drop due to frictional losses in the inlet and outlet channels is calculated from the axial momentum conservation equations as follows. Given a number of axial discretizations of the CPF inlet channel, cake+cat+wall and outlet channel $(j)$, there are $(j)$ 'streamlines' that can be conceived in the single channel representation as shown in Figure 3.14. Thus, there are $(j)$ ways of obtaining the absolute pressure at the inlet end of the inlet channel $\left(P_{1}\right)_{x=0}$, i.e., $P_{I_{1}}$ referring to Figure 3.14. The known boundary value for this problem is the measured exit pressure $\left(P_{2}\right)_{x=L}=P_{\text {exit }}$, i.e., $P_{O_{5}}$ referring to Figure 3.14 . 


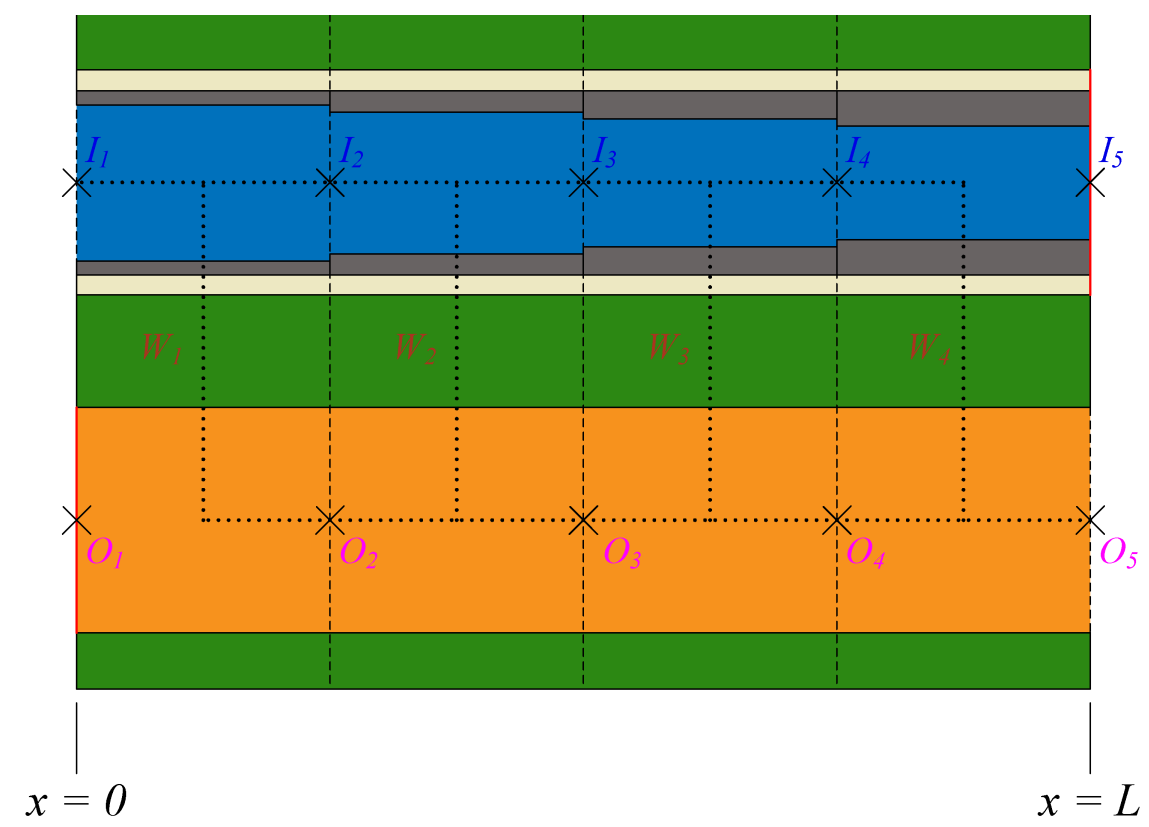

Figure 3.14: Schematic of streamlines (shown as dotted lines) for calculating pressure drop across CPF (Here, $j=4$ )

From the axial momentum conservation equation in the inlet channel (Equation (3.16)) and outlet channel (Equation (3.17)), the absolute pressure at any axial point can be calculated, knowing the absolute pressure at the point downstream of it by re-writing Equations (3.16) and (3.17) as:

$$
\begin{aligned}
& \frac{d P_{1}}{d x}=-\frac{d}{d x}\left(\rho_{1} v_{1}^{2}\right)-F \frac{\mu_{1} v_{1}}{a^{2}}\left(\frac{a^{*}}{a}\right)^{2} \\
& \frac{d P_{2}}{d x}=-\frac{d}{d x}\left(\rho_{2} v_{2}^{2}\right)-F \frac{\mu_{2} v_{2}}{a^{2}}
\end{aligned}
$$

or in discretized form,

$$
\begin{aligned}
& \left.P_{1}\right|_{j}=\left.P_{1}\right|_{j+1}+\left.\rho_{1} v_{1}^{2}\right|_{j+1}-\left.\rho_{1} v_{1}^{2}\right|_{j}+\left.F \Delta x \frac{\mu_{1} v_{1}}{a^{2}}\left(\frac{a^{*}}{a}\right)^{2}\right|_{j+\frac{1}{2}} \\
& \left.P_{2}\right|_{j}=\left.P_{2}\right|_{j+1}+\left.\rho_{2} v_{2}^{2}\right|_{j+1}-\left.\rho_{2} v_{2}^{2}\right|_{j+1}+\left.F \Delta x \frac{\mu_{2} v_{2}}{a^{2}}\right|_{j+\frac{1}{2}}
\end{aligned}
$$

From Figure 3.14, where the number of axial discretizations $j$ is equal to 4 , the streamlines are: 


$$
\begin{aligned}
& \text { 1. } O_{5} \underset{W_{4}}{\longrightarrow} I_{4} \underset{\text { eqn. (3.69a) }}{\longrightarrow} I_{3} \underset{\text { eqn. (3.69a) }}{\longrightarrow} I_{2} \underset{\text { eqn. (3.69a) }}{\longrightarrow} I_{1} \text {, } \\
& \text { 2. } O_{5} \underset{\text { eqn. (3.69b) }}{\longrightarrow} O_{4} \underset{W_{3}}{\longrightarrow} I_{3} \underset{\text { eqn. (3.69a) }}{\longrightarrow} I_{2} \underset{\text { eqn. (3.69a) }}{\longrightarrow} I_{1} \text {, } \\
& \text { 3. } O_{5} \underset{\text { eqn. (3.69b) }}{\longrightarrow} O_{4} \underset{\text { eqn. (3.69b) }}{\longrightarrow} O_{3} \underset{W_{2}}{\longrightarrow} I_{2} \underset{\text { eqn. (3.69a) }}{\longrightarrow} I_{1} \text {, and } \\
& \text { 4. } O_{5} \underset{\text { eqn. (3.69b) }}{\longrightarrow} O_{4} \underset{\text { eqn. (3.69b) }}{\longrightarrow} O_{3} \underset{\text { eqn. (3.69b) }}{\longrightarrow} O_{2} \underset{W_{1}}{\longrightarrow} I_{1} \text {. }
\end{aligned}
$$

In the CPF model version 4.0, at every sub-iteration of every time-step, $\left.P_{1}\right|_{x=0}$ is evaluated by starting out with $\left.P_{2}\right|_{x=L}=P_{\text {baro }}$ and then traversing through all possible streamlines. Thus, there are $j \max -1$ values of $\left.P_{1}\right|_{x=0}$ obtained where $j$ max -1 is the number of axial discretizations. These values are then used to compute relative differences of each value of $\left.P_{1}\right|_{x=0}$ to the average of the same, $\left.\overline{P_{1}}\right|_{x=0}$. The maximum relative difference between these values and the average are then compared to a user-specified tolerance, usually $\sim 0.001$. This is further explained in the convergence checks section (3.4.8).

\subsubsection{Solution Convergence Check}

Since the CPF model uses a sequential solution approach for different variables, a check for convergence is carried out at each time-step of the model. This is done by a submodel in the main body of the model source code which is shown as the trapezium titled 'Convergence reached?' in Figure E.1. The variables chosen for convergence check are: $P_{1}, P_{2}, v_{1}, v_{2}, v_{w}, T_{1}, T_{2}, T_{w}$ and $Y_{i}$.

As explained in section 3.3, when the simulation is initiated, the model calculates the time-step according to user-specified value of the variable $(d t)$. According to this value and total simulation time specified (stime), the initial time grid is constructed. The model traverses from one time-point to the next in this time grid (also known as 'time loop') in equal increments at first. At each one of these time points, the model calculates all variables being solved for at least twice (or 2 'sub-iterations'). Beginning at the second sub-iteration, the relative differences in current and previous values of variables that are checked for convergence are calculated. Mathematically, for the variable $X$,

$$
X_{\text {trend }}=\max \left(\operatorname{abs}\left(\frac{X_{\text {current }}-X_{\text {previous }}}{X_{\text {current }}}\right)\right)
$$

where $X_{\text {current }}$ is the current value of the variable $X$ and $X_{\text {previous }}$ is the previous value of the variable $X$.

For each type of variable (pressure, velocity, temperature, cake+cat+wall mole fraction and outlet channel mole fraction), user-defined convergence tolerances are input 
into the model, represented by the input parameters conv_tol_p, conv_tol_v, conv_tol_t, conv_tol_yi and conv_tol_y2i respectively (found in the input file input_parameters_v4_b04.dat).

Experiments were carried out to calibrate the CPF model described in this chapter. The next chapter describes these experiments and a procedure that was developed to calibrate the various input parameters to the CPF model using these experimental data. 


\section{Experimental DATa AND Model Calibration Procedure ${ }^{1}$}

The data to calibrate the CPF model were acquired on a Cummins $2007272 \mathrm{~kW}$ ISL engine with DOC and CPF aftertreatment devices. The test setup consisted of the ISL engine coupled to an eddy current dynamometer. Table 4.1 shows the specifications of DOC and CPF used in the testing. The data to calibrate the CPF model consists of passive oxidation and active regeneration experiments.

Table 4.1: Specifications of the after-treatment system used in the experiments [4,61]

\begin{tabular}{|l|c|c|l|}
\hline Property & DOC & CPF & Units \\
\hline Substrate material & Cordierite & Cordierite & \\
\hline Cell geometry & Square & Square & \\
\hline Diameter & 267 & 267 & {$[\mathrm{~mm}]$} \\
\hline Length & 102 & 305 & {$[\mathrm{~mm}]$} \\
\hline Total volume & 5.7 & 17.1 & {$[\mathrm{~L}]$} \\
\hline Cell density & $62(400)$ & $31(200)$ & {$\left[\mathrm{cells} / \mathrm{cm}^{2}(\mathrm{cpsi})\right]$} \\
\hline Channel width & 1.09 & 1.49 & {$[\mathrm{~mm}]$} \\
\hline Frontal area & 81 & 69 & {$[\%]$} \\
\hline Channel wall thickness & 0.114 & 0.305 & {$[\mathrm{~mm}]$} \\
\hline Wall density & NA & 0.45 & {$\left[\mathrm{~g} / \mathrm{cm}^{3}\right]$} \\
\hline Specific heat & NA & 891 & {$[\mathrm{~J} / \mathrm{kg} . \mathrm{K}]$} \\
\hline Thermal conductivity & NA & 0.84 & {$[\mathrm{~W} / \mathrm{m} . \mathrm{K}]$} \\
\hline Porosity & 35 & 52 & {$[\%]$} \\
\hline Mean pore size & NA & 13 & {$[\mathrm{~mm}]$} \\
\hline
\end{tabular}

Three different fuel types were used in the experiments - 100\% ultra low sulfur diesel (ULSD), 10\% soy-based methyl ester biodiesel blended with ultra low sulfur diesel (B10) and $20 \%$ biodiesel blended with ultra low sulfur diesel (B20). Table 4.2 shows the

\footnotetext{
${ }^{1}$ Parts of the material contained in this chapter have been published, or are currently under consideration for publication by SAE International.
} 
properties of the different fuels used during passive oxidation and active regeneration experiments.

Table 4.2: Specifications of the different fuels used in the experiments [54,55]

\begin{tabular}{|c|c|c|c|c|c|}
\hline \multicolumn{2}{|l|}{ Property } & ULSD & B10 & B20 & Units \\
\hline \multicolumn{2}{|c|}{ Sulfur content } & 7 & 4 & 4 & [ppm] \\
\hline \multicolumn{2}{|c|}{ Viscosity @ $40^{\circ} \mathrm{C}$} & 2.3 & 2.4 & 2.5 & {$[c S t]$} \\
\hline \multicolumn{2}{|c|}{ API gravity } & 35.6 & 36.5 & 35.5 & {$[]$.} \\
\hline \multicolumn{2}{|c|}{ Specific gravity } & 0.85 & 0.84 & 0.85 & {$[]$.} \\
\hline \multicolumn{2}{|c|}{ Cetane index } & 39.8 & 45.4 & 45.5 & {$[]$.} \\
\hline \multirow{2}{*}{ Distillation } & IBP & 168 & 168 & 168 & {$\left[{ }^{\circ} \mathrm{C}\right]$} \\
\hline & FBP & 340 & 343 & 345 & {$\left[{ }^{\circ} \mathrm{C}\right]$} \\
\hline \multicolumn{2}{|c|}{ Water content } & 92 & 415 & 553 & [ppm] \\
\hline \multicolumn{2}{|c|}{ ICP-AES for metals } & $<1$ & $<1$ & $<1$ & [ppm] \\
\hline \multicolumn{2}{|c|}{ IR for \% biodiesel } & 0 & 10 & 19 & [\%] \\
\hline \multicolumn{2}{|c|}{ Higher heating value } & 45.6 & 45.1 & 44.5 & {$[M J / k g]$} \\
\hline \multicolumn{2}{|c|}{ Lower heating value } & 42.8 & 42.5 & 42.0 & {$[M J / k g]$} \\
\hline \multicolumn{2}{|l|}{$\mathrm{H} / \mathrm{C}$ ratio } & 1.83 & 1.83 & 1.83 & {$[]$.} \\
\hline \multicolumn{2}{|l|}{$\mathrm{O} / \mathrm{C}$ ratio } & 0.00 & 0.01 & 0.02 & {$[]$.} \\
\hline
\end{tabular}

\subsection{Passive Oxidation (PO) Test Matrix}

The objective of the passive oxidation tests was to use the data to identify the kinetic parameters for the $\mathrm{NO}_{2}$-assisted PM oxidation in the CPF. The test setup, test matrix and instrumentation are explained in detail in references [54,61]. Figure 4.1 shows the total pressure drop across the CPF and the corresponding PM mass retained at the end of each stage. These passive oxidation experiments consisted of loading the CPF from a clean-state (after a clean-out via active regeneration for an extended period of time $(\sim 1 / 2 h r)$ ) to $\sim 2.5 \mathrm{~g} / \mathrm{l}$ PM loading in two stages (stage-1 and stage-2), followed by a ramp-up (RU) and passive oxidation phase (PO - typically an engine speed-load point at which there is a $\mathrm{PM} / N O_{x}$ ratio and $>300{ }^{\circ} \mathrm{C}$ temperature at engine-out/DOC-in) in which different percentages of PM oxidized were targeted, followed by a return to loading conditions (stage-3) for 0.5 hrs. which was then followed by a post-loading (stage-4) for a pre-determined period of time ( $1 \mathrm{hrs}$.). The deltailed experimental data can be found in reference [61].

After an analysis of the data collected, six passive oxidation experiments were used for the CPF model calibration as shown in Table 4.3. These experiments provided the data to determine the kinetic parameters for $\mathrm{NO}_{2}$-assisted PM oxidation. 


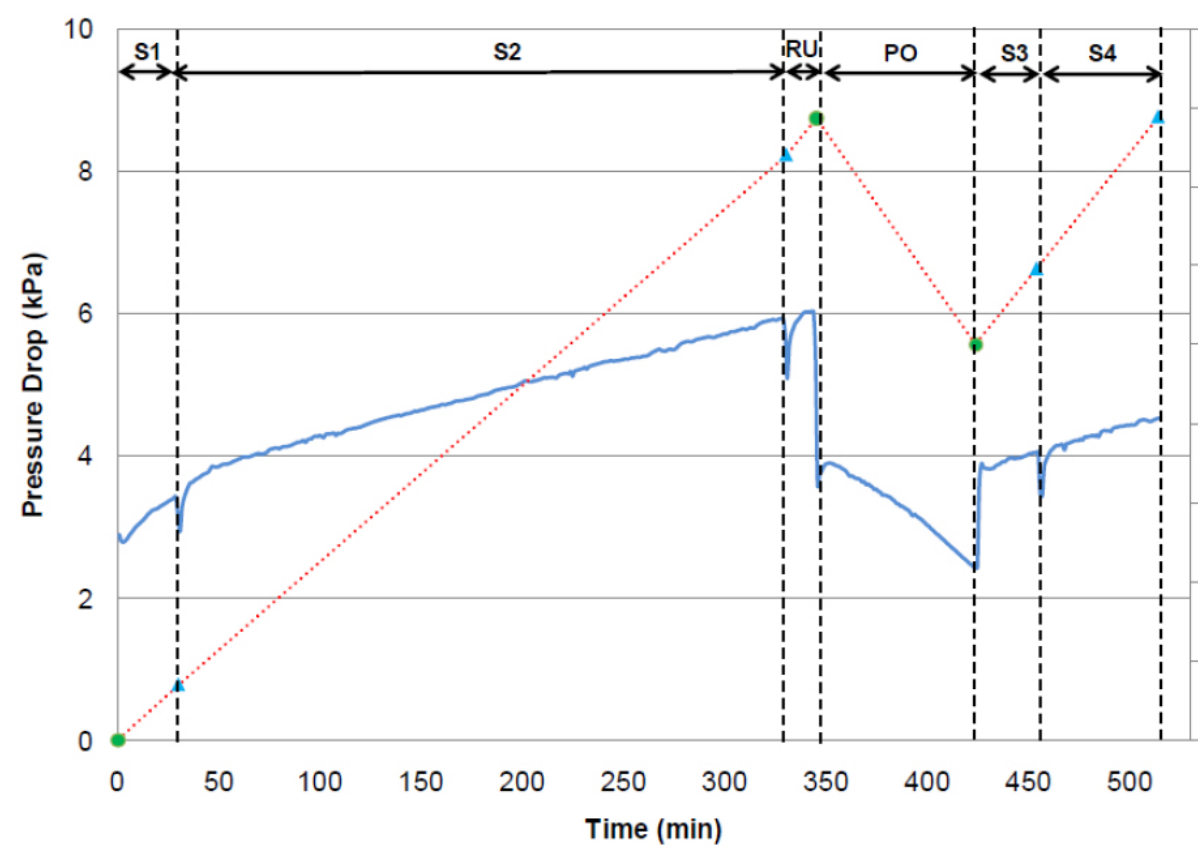

30 으 $-\triangle \mathrm{P} C P F$

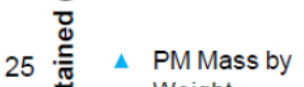
Weight

20

in ……est. PM

$15 \sum_{0}$ Mass

- PM Mass by

10

5 S1: Stage 1 loading S2: Stage 2 loading RU: Ramp-up

PO: Passive Oxidation S3: Stage 3 loading S4: Stage 4 loading

Figure 4.1: Overview of the various stages of the typical passive oxidation experiment - adapted from reference [54]

Table 4.3: Passive oxidation experiments used for the calibration of the CPF model

\begin{tabular}{|c|c|c|c|c|c|}
\hline \multirow{3}{*}{ No. } & \multirow{3}{*}{ Test ID } & \multicolumn{4}{|c|}{ Passive Oxidation } \\
\hline & & Temp. & Duration & $\begin{array}{l}\text { CPF inlet } \\
\mathrm{NO}_{2} / \mathrm{NO}_{x}\end{array}$ & $\begin{array}{r}\text { CPF inlet } \\
\mathrm{O}_{2}\end{array}$ \\
\hline & & {$\left[{ }^{\circ} \mathrm{C}\right]$} & [min.] & [ppm/ppm] & [\% Vol.] \\
\hline 1 & PO-B10-14 & 253 & 101 & $112 / 257$ & 12.6 \\
\hline 2 & PO-B10-15 & 355 & 81 & $101 / 194$ & 8.9 \\
\hline 3 & PO-B10-16 & 408 & 43 & $61 / 209$ & 7.1 \\
\hline 4 & PO-B10-17 & 356 & 80 & $90 / 178$ & 8.7 \\
\hline 5 & PO-B20-12 & 350 & 81 & $109 / 206$ & 9.4 \\
\hline 6 & PO-B20-13 & 403 & 42 & $64 / 204$ & 7.3 \\
\hline
\end{tabular}

\subsection{Active Regeneration (AR) Test Matrix}

The objective of the active regeneration experiments was to use the data to identify the kinetic parameters for the thermal $\left(\mathrm{O}_{2}\right)$ PM oxidation. The test setup, test matrix and instrumentation are explained in detail in references [4,55]. Figure 4.2 shows the total pressure drop across the $\mathrm{CPF}$ and the corresponding PM mass retained at the end of each stage. The active regeneration tests consists of stage 1 , stage 2 , loading ramp, active 
regeneration ramp, active regeneration stage, stage 3 and stage 4 . Stage 1 and 2 are the PM loading phase of the experiments similar to the passive oxidation tests. Stage 3 and 4 are similar to the post loading phase of the passive oxidation tests. During the loading and the active regeneration ramp, the engine was operated at the loading condition and the active regeneration engine condition for fifteen and ten minutes to stabilize the CPF temperature prior to active regeneration. Details of the experimental data can be found in reference [4].

After an analysis of the data collected, twelve active regeneration experiments were used for the CPF model calibration as shown in Table 4.4.

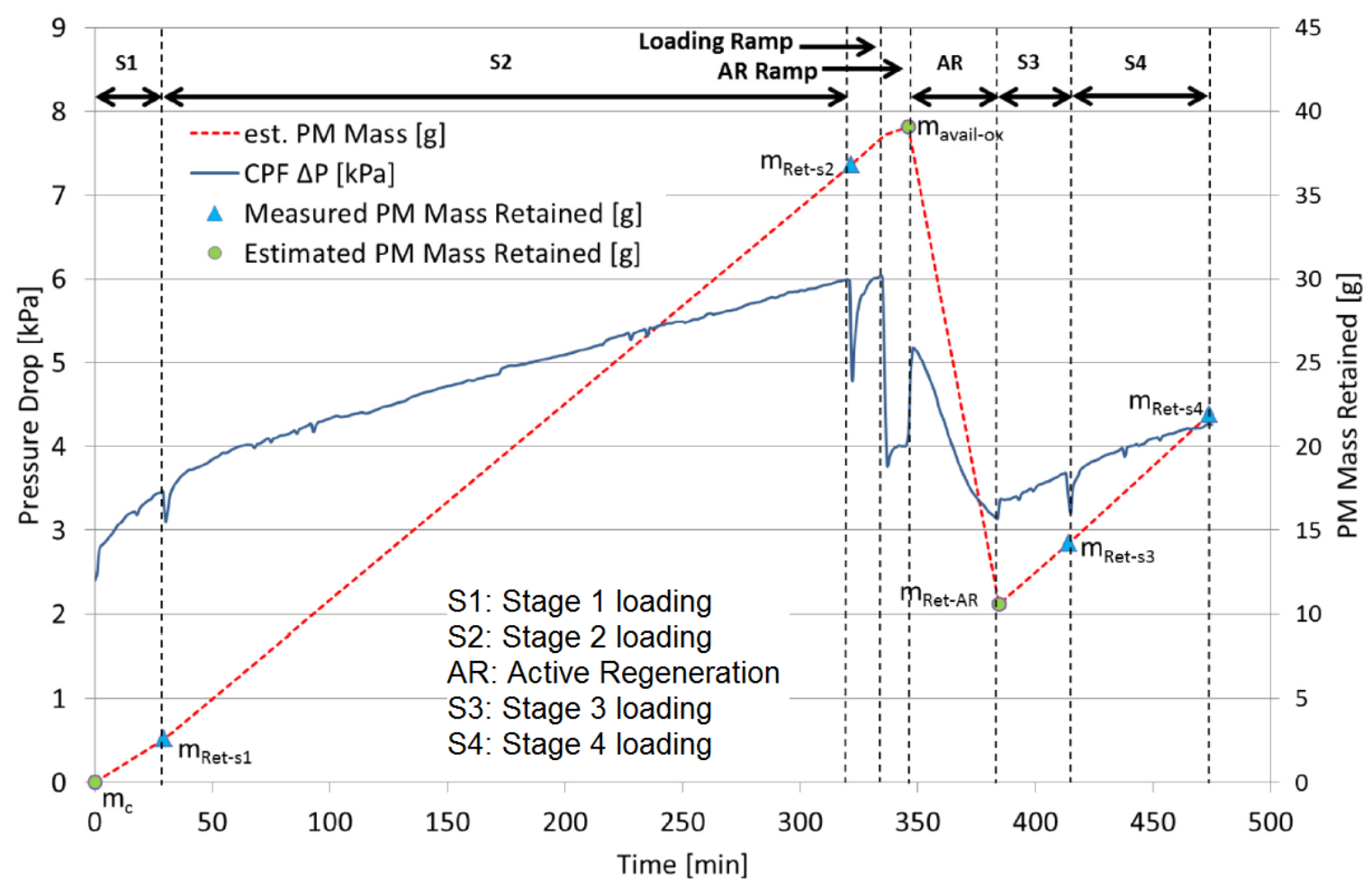

Figure 4.2: Overview of the various stages of the typical active regeneration experiment - adapted from reference [55] 
Table 4.4: Active regeneration experiments used for the calibration of the CPF model

\begin{tabular}{|c|c|c|c|c|c|}
\hline \multirow{3}{*}{ No. } & \multirow{3}{*}{ Test ID } & \multicolumn{4}{|c|}{ Active Regeneration } \\
\hline & & Temp. & Duration & $\begin{array}{c}\text { CPF inlet } \\
N O_{2} / N O_{x}\end{array}$ & $\begin{array}{r}\text { CPF inlet } \\
\mathrm{O}_{2}\end{array}$ \\
\hline & & {$\left[{ }^{\circ} \mathrm{C}\right]$} & [min.] & [ppm/ppm] & [\% Vol.] \\
\hline 1 & AR-ULSD-1 & 554 & 15 & $4 / 106$ & 7.2 \\
\hline 2 & AR-ULSD-2 & 581 & 6 & $7 / 141$ & 6.9 \\
\hline 3 & AR-ULSD-4 & 526 & 21 & $8 / 122$ & 7.6 \\
\hline 4 & AR-ULSD-5 & 524 & 22 & $6 / 131$ & 7.7 \\
\hline 5 & AR-ULSD-6 & 532 & 21 & $4 / 130$ & 7.7 \\
\hline 6 & AR-B10-1 & 530 & 26 & $4 / 119$ & 7.8 \\
\hline 7 & AR-B10-2 & 528 & 19 & $7 / 110$ & 7.8 \\
\hline 8 & AR-B10-4 & 554 & 15 & $3 / 124$ & 7.5 \\
\hline 9 & AR-B20-1 & 476 & 35 & $10 / 152$ & 8.8 \\
\hline 10 & AR-B20-2 & 503 & 39 & $4 / 138$ & 8 \\
\hline 11 & AR-B20-5 & 528 & 19 & $7 / 140$ & 8.2 \\
\hline 12 & AR-B20-6 & 531 & 16 & $10 / 124$ & 8.2 \\
\hline
\end{tabular}

\subsection{Procedure for the Model Calibration}

The CPF model requires the calibration parameters and time varying inputs to simulate the performance of the CPF. The time varying inputs are generated using the experimentally measured CPF inlet data. These are:

1. Mass flow rate of air, fuel and dozer fuel,

2. CPF inlet temperature,

3. Relative humidity, temperature and barometric pressure measured in the test cell, and

4. Concentrations of $\mathrm{HC}$ (represented by $\mathrm{C}_{12} \mathrm{H}_{24}$ ), $\mathrm{O}_{2}, \mathrm{CO}_{2}, \mathrm{CO}, \mathrm{NO}, \mathrm{NO}_{2}$ and PM at the CPF inlet.

The performance of the CPF model can be quantified by comparing:

- Total pressure drop across the CPF measured using differential pressure transducers,

- PM mass retained in the filter calculated from substrate weight measurements before and after each stage, 
- Overall filtration efficiency of the CPF calculated from upstream and downstream-CPF PM mass concentration measurements,

- Gas temperatures in the CPF and at the outlet of the CPF measured using K-type thermocouples, and

- $\mathrm{CPF}$ outlet concentrations of the gaseous species (specifically, $\mathrm{NO}, \mathrm{NO}_{2}, \mathrm{CO}$ and $H C$ ) measured by an emissions bench and CPF outlet PM concentrations.

The model output variables from a CPF simulation can be changed by changing the calibration parameters during calibration. The specific objective of the calibration of the CPF model was to determine the calibration parameters so as to simulate loading, passive oxidation and active regeneration (via in-cylinder and exhaust dosing) experiments using ULSD, B10 and B20 fuels.

Corrections to the input data were needed while preparing inputs for the high-fidelity $\mathrm{CPF}$ model to account for possible temperature and PM maldistribution in the CPF, exhaust flow maldistribution at the CPF inlet, and changing cake layer permeability. The correction factors also compensate for the phasing differences that may be present between the mass flow rate, temperature, or pressure drop signals. Specifically, a correction factor $(\mathrm{CF})$ was determined so as to eliminate the vertical shifts in CPF flow resistance calculated from measured $\mathrm{CPF}$ pressure drops, temperatures and mass flow rates of air and fuel. The methodology used to determine the correction factors for each active regeneration experiment is described in reference [3]. The correction factors for the eighteen experiments are as shown in Table A1.

The overall approach to calibration of the model was to:

1. Determine one set of filtration parameters that simulated the experimental pressure drop and filtration efficiency during loading (stage-1 and stage-2) for passive oxidation and active regeneration tests,

2. Determine the kinetic parameters of reactions involving gaseous species to simulate the CPF outlet gaseous species concentrations specifically of $\mathrm{C}_{12} \mathrm{H}_{24}, \mathrm{CO}, \mathrm{NO}$ and $\mathrm{NO}_{2}$,

3. Determine the kinetic parameters related to PM oxidation reactions, specifically $\mathrm{NO}_{2}$-assisted PM oxidation reaction from the passive oxidation experiments to simulate PM mass retained at the end of stage-2, stage- 3 and stage- 4 of all passive oxidation experiments,

4. Use the $\mathrm{NO}_{2}$-assisted PM oxidation reaction kinetic parameters and determine the thermal $\left(\mathrm{O}_{2}\right)$ PM oxidation kinetic parameters from the active regeneration 
experiments so as to simulate the PM mass retained at the end of stage-2, stage- 3 and stage- 4 of all active regeneration experiments, and

5. Use Arrhenius plots to optimize the $\mathrm{NO}_{2}$-assisted and thermal $\left(\mathrm{O}_{2}\right)$ PM oxidation kinetic parameters.

\subsubsection{Filtration}

Table 4.5 gives a description of the calibration parameters that were considered to be varied to get agreement with the experimental pressure drop and filtration efficiency data.

Filtration-related calibration parameters were adjusted to simulate experimental total pressure drop and filtration efficiency calculated from PM concentration measurements upstream and downstream of the CPF during loading stage- 2 of passive oxidation and active regeneration experiments. These values are as shown in Table J.1 (pressure drop) and Table J.3 (filtration efficiency).

\subsubsection{Catalytic Reaction Kinetics}

Table 4.6 gives a description of the catalytic reaction kinetic parameters that were considered to be varied to get agreement with the experimental outlet concentrations of $\mathrm{C}_{12} \mathrm{H}_{24}, \mathrm{CO}, \mathrm{NO}$ and $\mathrm{NO}_{2}$.

The activation energies for $N O, C O$ and $H C$ oxidation reactions were obtained from reference [73] for three-way oxidation reactions in a 1-D DOC model. The corresponding pre-exponential factors were obtained by simulating experimental outlet $\mathrm{NO}, \mathrm{NO}_{2}$, $\mathrm{CO}$ and $\mathrm{C}_{12} \mathrm{H}_{24}$ concentrations during the passive oxidation and active regeneration experiments and are shown in Table J.5 in Appendix J.

For calibrating $\mathrm{CPF}$ outlet $\mathrm{NO}$ and $\mathrm{NO}_{2}$ concentrations at different $\mathrm{NO}_{x}$ /temperature points, another parameter that was calibrated was the tortuosity of the PM cake layer $\left(\tau_{\text {cake }}\right)$. This parameter was optimized along with the pre-exponential factor for NO oxidation reaction to obtain model-predicted $\mathrm{NO}_{2}$ concentrations within $\pm 10 \mathrm{ppm}$ of experimental measurements as shown in Table J.5 in Appendix J. The optimized values of kinetic parameters of $\mathrm{NO}$ oxidation reaction are shown later in the Results and Discussions chapter (Chapter 5). 


\subsubsection{PM Oxidation Kinetics}

Table 4.7 gives a description of the PM oxidation reaction kinetic parameters that were considered to be varied to get agreement with the experimental PM mass retained and CPF pressure drop.

For calibrating the PM kinetics associated with the thermal and $\mathrm{NO}_{2}$-assisted mechanisms in the PM cake layer and substrate wall, the following steps were followed:

1. Initial values of activation energies and pre-exponential factors for the $\mathrm{NO}_{2}$-assisted PM oxidation reaction were determined by an optimization computation with experimental data from the passive oxidation experiments, grouped according to fuel type (ULSD, B10 and B20) as shown in Table 4.8. Initial values of kinetic parameters of thermal $\left(\mathrm{O}_{2}\right)$ PM oxidation were fixed at values obtained from analysis of experimental data in reference [4]. Pre-exponential factors of these reactions according to the fuel and activation energy are shown in Table 4.8.

2. Since the CPF inlet PM concentrations were known to be varying from experiment to experiment, specific values of CPF inlet PM concentrations were determined for stage- 1 and stage- 2 for all passive oxidation and active regeneration experiments such that the original values of $\mathrm{NO}_{2}$-assisted PM oxidation kinetic parameters in the PM cake layer $\left(A_{\mathrm{NO}_{2}, \text { cake }}\right)$ could be used to simulate end-of-stage-2 PM mass retained within $(-1.1 /+0.9 \mathrm{~g})$ as shown in Table J.2 in Appendix J. The specific values of $\mathrm{CPF}$ inlet PM concentrations used for stage- 1 and stage- 2 of all passive oxidation and active regeneration experiments are as shown in Table H.2 in Appendix H. The pre-exponential factors of $\mathrm{NO}_{2}$-assisted PM oxidation in the wall during loading $\left(A_{\mathrm{NO}_{2}, \text { wall }}\right)$ were adjusted to simulate the slope of the loading pressure drop.

3. Using the values of $\mathrm{NO}_{2}$-assisted PM oxidation kinetic parameters in the cake and wall, the active regeneration experiments were simulated. Specifically, the pre-exponential factors of thermal PM oxidation reaction in the PM cake layer $\left(A_{\text {th,cake }}\right)$ were calibrated for all active regeneration experiments to simulate end-of-stage-3 PM mass retained in the filter (as shown in Table J.2) and the pre-exponential factors of thermal PM oxidation reaction in the wall during active regeneration $\left(A_{t h, \text { wall }}\right)$ were adjusted to simulate the slope of the pressure drop during active regeneration.

4. The $\mathrm{NO}_{2}$-assisted PM oxidation kinetic parameters of all passive oxidation experiments and thermal PM oxidation reaction kinetic parameters of all active regeneration experiments were analyzed using Arrhenius plots and optimized values of pre-exponential factors and activation energies were arrived at from these Arrhenius plots for all passive oxidation and active regeneration experiments. 
The model calibration procedure explained in this chapter was used with the experimental data described in Tables 4.3 and 4.4 to calibrate the model. The next chapter applies this procedure to determine the parameters needed for the model calibration. The detailed results for a PO and AR case are then presented along with the differences between the experimental data and the model in Appendix $\mathrm{J}$ for all eighteen experimental cases. 
Table 4.5: Filtration-related input parameters considered for the CPF model calibration

\begin{tabular}{|c|c|c|}
\hline Parameter & Description & $\begin{array}{l}\text { Expt. Data Used to } \\
\text { Calibrate Parameter }\end{array}$ \\
\hline$D$ & Diameter of substrate & \multirow{7}{*}{$\begin{array}{l}\text { Geometry/specifications of } \\
\text { CPF }\end{array}$} \\
\hline$L$ & Length of substrate & \\
\hline$w_{s}$ & Thickness of substrate wall & \\
\hline$n_{\text {cell }}$ & $\begin{array}{l}\text { Number of inlet channels in the } \\
\text { substrate }\end{array}$ & \\
\hline$\rho_{s}$ & Bulk density of substrate wall & \\
\hline$c_{p, s}$ & $\begin{array}{l}\text { Specific heat capacity of substrate } \\
\text { wall }\end{array}$ & \\
\hline$\lambda_{s}$ & $\begin{array}{l}\text { Thermal conductivity of substrate } \\
\text { wall }\end{array}$ & \\
\hline$k_{s, 0}$ & Initial permeability of substrate wall & Clean pressure drop \\
\hline$k_{\text {trans }}$ & $\begin{array}{l}\text { Transition permeability of substrate } \\
\text { wall }\end{array}$ & $\begin{array}{l}\text { Duration of deep-bed } \\
\text { filtration }\end{array}$ \\
\hline$\epsilon_{s, 0}$ & Initial porosity of substrate wall & \multirow{3}{*}{ Deep-bed pressure drop } \\
\hline $\begin{array}{l}d_{\text {pore }, 0} \\
C_{1, \rho_{p w}}\end{array}$ & $\begin{array}{l}\text { Mean pore size of substrate wall } \\
\text { First constant in wall packing density } \\
\text { calculation }\end{array}$ & \\
\hline$C_{2, \rho_{p w}}$ & $\begin{array}{l}\text { Second constant in wall wall packing } \\
\text { density calculation }\end{array}$ & \\
\hline$w_{\text {cat }}$ & Thickness of catalyst washcoat & \multirow[b]{2}{*}{ Catalyst specifications } \\
\hline$d_{\text {pen }}$ & $\begin{array}{l}\text { Depth of penetration of catalyst into } \\
\text { the substrate wall }\end{array}$ & \\
\hline$\alpha_{p, 0}$ & Initial solidosity of PM cake layer & $\begin{array}{l}\text { Calculate according to Peclet } \\
\text { number [72] }\end{array}$ \\
\hline$k_{p, 0}$ & Initial permeability of PM cake layer & $\begin{array}{l}\text { Slope of cake filtration } \\
\text { pressure drop }\end{array}$ \\
\hline$\lambda_{p}$ & $\begin{array}{l}\text { Thermal conductivity of PM cake } \\
\text { layer }\end{array}$ & \multirow[t]{2}{*}{ Constants } \\
\hline$c_{p, p}$ & $\begin{array}{l}\text { Specific heat capacity of PM cake } \\
\text { layer }\end{array}$ & \\
\hline$A_{\eta}$ & $\begin{array}{l}\text { PM cake filtration efficiency } \\
\text { parameter }\end{array}$ & $\begin{array}{l}\text { Total filtration efficiency } \\
\text { during steady-state loading }\end{array}$ \\
\hline$(h A)_{a m b}$ & $\begin{array}{l}\text { Heat transfer coefficient for } \\
\text { convectional heat transfer with } \\
\text { ambient }\end{array}$ & $\begin{array}{l}\text { CPF outlet temperature } \\
\text { during PO/AR }\end{array}$ \\
\hline
\end{tabular}


Table 4.6: Catalytic reaction kinetic parameters considered for the CPF model calibration

\begin{tabular}{|c|c|c|c|}
\hline & Parameter & Description & $\begin{array}{l}\text { Expt. Data Used to } \\
\text { Calibrate Parameter }\end{array}$ \\
\hline \multirow{5}{*}{ 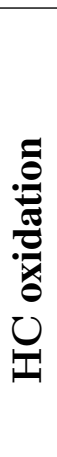 } & $A_{H C}$ & Pre-exponential factor & \multirow{4}{*}{$\begin{array}{l}\text { HC conversion efficiency, } \\
\text { CPF outlet temperature }\end{array}$} \\
\hline & $E a_{H C}$ & Activation energy & \\
\hline & $x_{H C}^{G}$ & $\begin{array}{l}\text { Temperature order of } \\
\text { dependence of inhibition } \\
\text { factor }\end{array}$ & \\
\hline & $K_{a 0,(i=1-4)}$ & $\begin{array}{l}\text { Adsorption factors of } \\
\text { inhibition factor }\end{array}$ & \\
\hline & $\Delta H_{a,(i=1-4)}$ & $\begin{array}{l}\text { Adsorption heats of } \\
\text { inhibition factor }\end{array}$ & Literature [51] \\
\hline \multirow{5}{*}{ 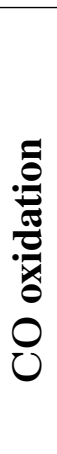 } & $A_{C O}$ & Pre-exponential factor & \multirow{4}{*}{ CO conversion efficiency } \\
\hline & $E a_{C O}$ & Activation energy & \\
\hline & $x_{C O}^{G}$ & $\begin{array}{l}\text { Temperature order of } \\
\text { dependence of inhibition } \\
\text { factor }\end{array}$ & \\
\hline & $K_{a 0,(i=5-8)}$ & $\begin{array}{l}\text { Adsorption factors of } \\
\text { inhibition factor }\end{array}$ & \\
\hline & $\Delta H_{a,(i=5-8)}$ & $\begin{array}{l}\text { Adsorption heats of } \\
\text { inhibition factor }\end{array}$ & Literature [51] \\
\hline \multirow{5}{*}{ 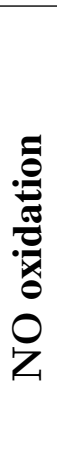 } & $A_{N O}$ & Pre-exponential factor & \multirow{4}{*}{ NO conversion efficiency } \\
\hline & $E a_{N O}$ & Activation energy & \\
\hline & $x_{N O}^{G}$ & $\begin{array}{l}\text { Temperature order of } \\
\text { dependence of inhibition } \\
\text { factor }\end{array}$ & \\
\hline & $K_{a 0,(i=9-12)}$ & $\begin{array}{l}\text { Adsorption factors of } \\
\text { inhibition factor }\end{array}$ & \\
\hline & $\Delta H_{a,(i=9-12)}$ & $\begin{array}{l}\text { Adsorption heats of } \\
\text { inhibition factor }\end{array}$ & Literature [51] \\
\hline
\end{tabular}


Table 4.7: PM oxidation kinetic parameters considered for the CPF model calibration

\begin{tabular}{|c|c|c|c|c|}
\hline & Location & Parameter & Description & $\begin{array}{l}\text { Expt. Data Used to } \\
\text { Calibrate Parameter }\end{array}$ \\
\hline \multirow{4}{*}{$\bigcirc^{N}$} & \multirow{2}{*}{ Cake } & $A_{\mathrm{NO}_{2}, \text { cake }}$ & Pre-exponential factor & \multirow{2}{*}{$\begin{array}{l}\text { Mass of PM retained after } \\
\text { passive oxidation }\end{array}$} \\
\hline & & $E a_{\mathrm{NO}_{2}, \text { cake }}$ & Activation energy & \\
\hline & \multirow{2}{*}{ Wall } & $A_{\mathrm{NO}_{2}, \text { wall }}$ & Pre-exponential factor & \multirow{2}{*}{$\begin{array}{l}\text { Pressure drop during passive } \\
\text { oxidation }\end{array}$} \\
\hline & & $E a_{N_{2}, \text { wall }}$ & Activation energy & \\
\hline \multirow{4}{*}{ } & \multirow{2}{*}{ Cake } & $A_{\text {th,cake }}$ & Pre-exponential factor & \multirow{2}{*}{$\begin{array}{l}\text { Mass of PM retained after } \\
\text { active regeneration }\end{array}$} \\
\hline & & $E a_{\text {th,cake }}$ & Activation energy & \\
\hline & \multirow{2}{*}{ Wall } & $A_{\text {th,wall }}$ & Pre-exponential factor & \multirow{2}{*}{$\begin{array}{l}\text { Pressure drop during active } \\
\text { regeneration }\end{array}$} \\
\hline & & $E a_{\text {th,wall }}$ & Activation energy & \\
\hline
\end{tabular}

Table 4.8: Initial values of kinetic parameters of PM oxidation reactions determined by optimization computation of $\mathrm{NO}_{2}$-assisted PM oxidation and from analysis of experimental data in reference [4] used for calibration of high-fidelity CPF model

\begin{tabular}{|l|r|r|r|l|}
\hline Parameter & ULSD & B10 & B20 & Units \\
\hline$A_{\mathrm{NO}_{2}}$ & 0.21 & 0.06 & 0.015 & {$[\mathrm{~m} / \mathrm{s}]$} \\
\hline$E a_{\mathrm{NO}_{2}}$ & 74.1 & 68.3 & 59.4 & {$[\mathrm{~kJ} / \mathrm{gmol}]$} \\
\hline$A_{t h}$ & 0.942 & 1.17 & 1.93 & {$[\mathrm{~m} / \mathrm{s}]$} \\
\hline$E a_{t h}$ & \multicolumn{3}{|c|}{139} & {$[\mathrm{~kJ} / \mathrm{gmol}]$} \\
\hline
\end{tabular}




\section{Results AND Discussion ${ }^{1}$}

The CPF model was calibrated using experimental data from 6 PO and 12 AR experiments. As a result of calibration of the model, all calibration parameters that need to be specified as inputs to the model were determined. The following sections of this chapter discuss the parameters obtained and the performance of the CPF simulated using these parameters compared to the experimental data obtained during $\mathrm{PO}$ and $\mathrm{AR}$ experiments. A comparison of the measured experimental CPF performance data to the model results from one PO and one AR experiment are presented in the chapter with additional comparisons from all PO and AR experiments have been given in Appendix J.

\subsection{Input Parameters Obtained from Model Calibration}

\subsubsection{Filtration Parameters}

Filtration parameters determined as a result of model calibration for the eighteen experiments are shown in Table I.1. The filtration parameters that varied across different experiments are: $k_{s, 0}, k_{\text {trans }}, C_{1, \rho_{p w}}$ and $C_{2, \rho_{p w}}$ as shown in Table I.1. The initial substrate permeability $k_{s, 0}$ needed to be calibrated for 2 of the 18 experiments to match the initial pressure drop; otherwise a constant value of $1.19 \times 10^{-13} \mathrm{~m}^{2}$ was used for all experiments in this study. The transition permeability $k_{\text {trans }}$ needed to be calibrated for all experiments to control the time point at which PM cake layer starts to form. The average of 17 out of the 18 experiments for $k_{\text {trans }}$ was $7.72 \times 10^{-14} \mathrm{~m}^{2}$ with a standard deviation of $0.235 \mathrm{x}$ $10^{-14} \mathrm{~m}^{2}$. One case which was different from all others in both values of $k_{s, 0}$ and $k_{\text {trans }}$ was AR-B20-1, which had no deep-bed filtration region and therefore had a lower overall pressure drop than the loading stages (stage-1 and stage-2) of all other passive oxidation and active regeneration experiments. Correspondingly, a $k_{s, 0}$ value of $1.15 \times 10^{-13} \mathrm{~m}^{2}$ and $k_{\text {trans }}$ of $1.19 \times 10^{-13} \mathrm{~m}^{2}$ was used for AR-B20-1 such that in the simulation, PM cake layer

\footnotetext{
${ }^{1}$ Parts of the material contained in this chapter have been published, or are currently under consideration for publication by SAE International.
} 
filtration starts from the initiation of the experiment itself, whereby deep bed filtration region is by-passed. The average value of $C_{1, \rho_{p w}}$ used for all 18 experiments was $2.20 \mathrm{~m}^{-3}$ (with only 4 cases different from $2.20 \mathrm{~m}^{-3}$ ) and standard deviation was $0.05 \mathrm{~m}^{-3} . C_{2, \rho_{p w}}$ for all 18 experiments had an average value of $1.52 \mathrm{~kg} / \mathrm{m}^{3}$ (with only 5 cases different from $1.48 \mathrm{~kg} / \mathrm{m}^{3}$ ) and standard deviation of $0.07 \mathrm{~kg} / \mathrm{m}^{3}$.

Since the goal of model calibration was to obtain a single set of input parameters including the filtration parameters, a set of filtration-related input parameters that simulates the experimental data for the most number of experiments was chosen as the representative input parameters set as shown in Table 5.1. In addition to the filtration parameters that are shown in Table 4.5, another variable that was varied during each simulation of passive oxidation phase was the permeability of the PM cake layer $\left(k_{p}\right)$. In order to simulate the experimental pressure drop and the PM oxidation, it was observed that the PM cake layer permeability had to be time-varying and showed a consistent trend across 5 of the 6 passive oxidation experiments (the $6^{\text {th }}$ experiment being PO-B10-14, the test-case with no significant PM oxidation during passive oxidation). The values used for the cake permeability are shown in Figure I.1. All permeability values start at a constant value $\left(0.7 \times 10^{-14} \mathrm{~m}^{2}\right)$ for the entire duration of stage- 1 and stage-2, and for the part of the passive oxidation phase where PM conversion is less than 25\% (PM conversion at any point in passive oxidation phase is defined as percentage of PM available for oxidation oxidized from start of passive oxidation till that point in time). After that point, the permeability of PM cake layer increases linearly till the end of passive oxidation $\left(1.211 \times 10^{-14}-2.171 \times 10^{-14} \mathrm{~m}^{2}\right)$. After the start of post-loading, PM cake layer permeability exponentially decreases to a different steady-state value that is always higher than the initial value used $\left(0.87 \times 10^{-14}-1.07 \times 10^{-14} \mathrm{~m}^{2}\right)$. It is thought that this change in cake permeability is likely due to the rate of PM oxidation changing the porosity of the cake. This was not seen during active regeneration since the oxidation of the cake layer is very rapid due to the temperature being greater than $500{ }^{\circ} \mathrm{C}$.

\subsubsection{Catalytic Reaction Kinetics}

As a result of model calibration, kinetic parameters of catalytic three-way reactions were obtained by simulating model output species concentration values at CPF outlet with experimentally measured concentrations during each experiment. Of these, $\mathrm{NO}_{2}$ kinetics were the most important since the production of $\mathrm{NO}_{2}$ in the catalyst washcoat layer could significantly impact back-diffusion of $\mathrm{NO}_{2}$ and hence PM oxidation in the PM cake layer. A detailed comparison of model and experimental $\mathrm{NO}_{2}$ concentrations for all $\mathrm{PO}$ and AR experiments is shown in Table J.5. The resulting $\mathrm{NO}_{2}$ kinetics and variables involved in the corresponding inhibition factors are as shown in Table 5.2.

In addition to these kinetic parameters, the other input parameters to the CPF model that 
Table 5.1: Single set of filtration parameters obtained from CPF model calibration

\begin{tabular}{|c|c|c|}
\hline Parameter & Value & Units \\
\hline \multicolumn{3}{|l|}{ Geometry } \\
\hline$D$ & 10.5 & [in.] \\
\hline$L$ & 12 & [in.] \\
\hline$n_{\text {cell }}$ & 8659 & {$[]$.} \\
\hline \multicolumn{3}{|l|}{ Substrate Wall } \\
\hline$w_{s}$ & 12 & [Mil] \\
\hline$\rho_{s}$ & 450 & {$\left[\mathrm{~kg} / \mathrm{m}^{3}\right]$} \\
\hline$c_{p, s}$ & 891 & {$[\mathrm{~J} / \mathrm{kg} . \mathrm{K}]$} \\
\hline$\lambda_{s}$ & 1 & {$[W / m . K]$} \\
\hline$k_{s, 0}\left(\times 10^{-13}\right)$ & 1.19 & {$\left[m^{2}\right]$} \\
\hline$k_{\text {trans }}\left(\times 10^{-13}\right)$ & 0.70 & {$\left[m^{2}\right]$} \\
\hline$\epsilon_{s, 0}$ & 0.5 & {$[]$.} \\
\hline$d_{\text {pore }, 0}$ & 17.5 & {$[\mu m]$} \\
\hline \multicolumn{3}{|l|}{ Wall PM } \\
\hline$C_{1, \rho_{p w}}$ & 2.20 & {$\left[1 / m^{3}\right]$} \\
\hline$C_{2, \rho_{p w}}$ & 1.48 & {$\left[\mathrm{~kg} / \mathrm{m}^{3}\right]$} \\
\hline \multicolumn{3}{|l|}{ Catalyst } \\
\hline$w_{\text {cat }}$ & 20 & {$[\mu m]$} \\
\hline$d_{\text {pen }}$ & 0 & {$[\mu \mathrm{m}]$} \\
\hline \multicolumn{3}{|l|}{ PM cake } \\
\hline$\alpha_{p, 0}$ & 0.05 & {$[]$.} \\
\hline$k_{p, 0}\left(\times 10^{-15}\right)$ & 7 & {$\left[m^{2}\right]$} \\
\hline$\lambda_{p}$ & 2.1 & {$[W / m . K]$} \\
\hline$c_{p, p}$ & 1510 & {$[\mathrm{~J} / \mathrm{kg} . \mathrm{K}]$} \\
\hline$A_{\eta}\left(\times 10^{-2}\right)$ & 95 & {$[]$.} \\
\hline \multicolumn{3}{|l|}{ Heat Transfer } \\
\hline$(h A)_{a m b}$ & 0.05 & {$[W / K]$} \\
\hline
\end{tabular}


Table 5.2: Single set of kinetic parameters for catalytic reactions obtained from CPF model calibration

\begin{tabular}{|c|c|c|c|c|c|c|c|}
\hline Parameter & \multicolumn{2}{|c|}{ HC oxidation } & \multicolumn{2}{|c|}{ CO oxidation } & \multicolumn{2}{|c|}{ NO oxidation } & Units \\
\hline$A_{j}$ & \multicolumn{2}{|c|}{$6.0 \times 10^{10}$} & \multicolumn{2}{|c|}{$5.0 \times 10^{10}$} & \multicolumn{2}{|c|}{$2.5 \times 10^{11}$} & {$[*]$} \\
\hline$E a_{j}$ & \multicolumn{2}{|c|}{39.0} & \multicolumn{2}{|c|}{43.5} & \multicolumn{2}{|c|}{46.4} & {$[\mathrm{~kJ} / \mathrm{mol}]$} \\
\hline \multirow{2}{*}{$\begin{array}{l}\text { Inhibition } \\
\text { parameters }\end{array}$} & $K a_{0, i}$ & $\Delta H_{a, i}$ & $K a_{0, i}$ & $\Delta H_{a, i}$ & $K a_{0, i)}$ & $\Delta H_{a, i}$ & \\
\hline & \multicolumn{2}{|c|}{$(i=1-4)$} & \multicolumn{2}{|c|}{$(i=5-8)$} & \multicolumn{2}{|c|}{$(i=9-12)$} & \\
\hline Units $\rightarrow$ & [.] & [J/kmol] & {$[]$.} & [J/kmol] & {$[]$.} & [J/kmol] & \\
\hline$K_{a,(i=1,5,9)}$ & 0.0665 & 7995.65 & 0.0665 & 7995.65 & 0.0665 & 7995.65 & \\
\hline$K_{a,(i=2,6,10)}$ & 0.2080 & 3004.15 & 0.2080 & 3004.15 & 0.2080 & 3004.15 & \\
\hline$K_{a,(i=3,7,11)}$ & 0 & 96594.8 & 0 & 96594.8 & 0 & 96594.8 & \\
\hline$K_{a,(i=4,8,12)}$ & $2 \mathrm{E}+05$ & -31058 & $2 \mathrm{E}+05$ & -31058 & $2 \mathrm{E}+06$ & -31058 & \\
\hline
\end{tabular}

were varied to get agreement with outlet $\mathrm{NO}_{2}$ concentrations of both passive oxidation and active regeneration experiments were the temperature order of dependence of the inhibition factor of NO oxidation reaction $\left(x_{N O}^{G}\right)$ as given in Equation (3.40) and shown in Table 4.6. The value of $\left(x_{N O}^{G}\right)$ was calibrated along with the kinetic parameters $\left(A_{N O}\right.$ and $\left.E a_{N O}\right)$ and inhibition parameters $\left(K_{a 0, i}(i=9-12)\right)$ to simulate CPF outlet NO2 concentratiosn during loading, PO/AR and post-loading stages of all eighteen PO and AR experiments. The corresponding comparisons of outlet $\mathrm{NO}_{2}$ concentrations between model and experimental data for all experiments are as shown in Table J.5. The tortousity of the PM cake layer was another parameter that was calibrated for all eighteen experiments. This parameter determines the effective diffusivity of the gaseous species in the PM cake layer as shown in Equation D.5 in Appendix D. Calibration of this variable was carried out so as to simulate $\mathrm{CPF}$ outlet $\mathrm{NO}_{2}$ concentrations for all eighteen experiments using the same set of kinetic parameters and inhibition parameters. The calibrated value of this variable was 1 . This variable will have a significant impact on the back-diffusion of $\mathrm{NO}_{2}$ as concluded by reference [59] as described in Section 2.4. Published values of this variable for diesel PM cake layer from medium duty engines are not available in the open literature. Therefore, further experimental investigations are needed to determine the effective diffusivities accurately.

\subsubsection{PM Oxidation Kinetics}

After calibrating the model for all passive oxidation experiments as shown in Table 4.3, the natural logarithms of individual $\mathrm{NO}_{2}$-assisted reaction rate constants were plotted versus the inverse of the average absolute temperature (Arrhenius plots). Figures 5.1 and 5.2 show the Arrhenius plots for $\mathrm{NO}_{2}$-assisted PM oxidation in the PM cake layer and substrate wall respectively, for all passive oxidation experiments, grouped by fuel-type 
(green - B10, blue - B20). This showed that for both B10 and B20 fuel-types, the values of pre-exponential factors and activation energies for $\mathrm{NO}_{2}$-assisted oxidation in PM cake layer that were used as initial values could still be used for loading as well as passive oxidation as shown in Table 5.3. Also, the kinetics of $\mathrm{NO}_{2}$-assisted PM oxidation in the wall were obtained as shown in Table 5.3. It was observed that the same activation energies could be used for wall and cake, and that in general, wall pre-exponential factors were higher than cake pre-exponential factors given the same fuel type. This could be due to the fact that the wall pre-exponential factors are from a simpler model of the wall and are adjusted such that the model pressure drop slopes simulate the experimental data, while the cake kinetics are calibrated such that the model PM mass retained simulate the experimentally measured values.

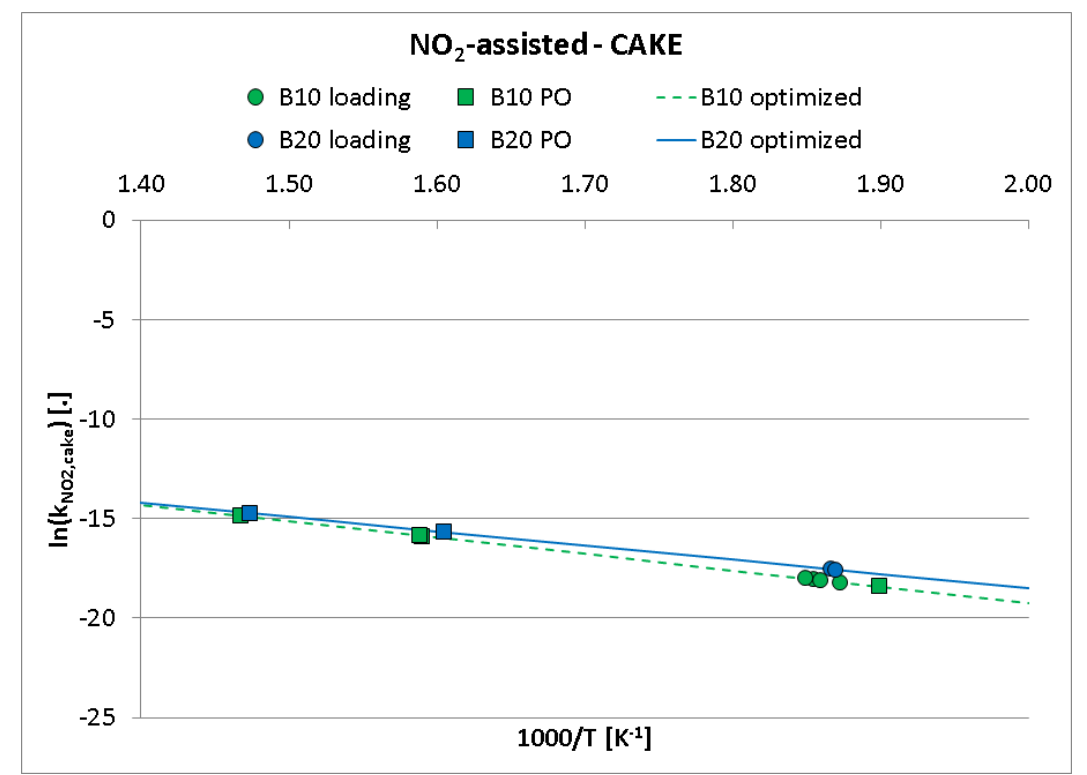

Figure 5.1: Arrhenius plot of $\mathrm{NO}_{2}$-assisted PM oxidation reaction in PM cake layer shown for all passive oxidation experiments

Due to the lack of experimental data for passive oxidation using ULSD fuel-type, the $\mathrm{NO}_{2}$-assisted PM kinetics for ULSD were obtained by calibrating the model PM mass retained to the loading phases of all ULSD AR experiments (as shown in Table J.2). The $\mathrm{NO}_{2}$-assisted PM kinetics obtained thus are as shown in Table 5.3. The calibrated value of activation energy of $\mathrm{NO}_{2}$-assisted oxidation of ULSD-derived PM agrees with the reported value of $\mathrm{NO}_{2}$-assisted oxidation of ULSD-derived PM [51] as shown in Table 2.1. Activation energies of other fuels (B10 and B20) also are in the expected range, although values of these are not available in the open literature.

Having obtained $\mathrm{NO}_{2}$-assisted PM kinetics, the next step was to determine the thermal $\left(\mathrm{O}_{2}\right)$ PM kinetics. The pre-exponential factors and activation energies for $\mathrm{NO}_{2}$-assisted oxidation were used and the model calibrated to all active regeneration experiments to 


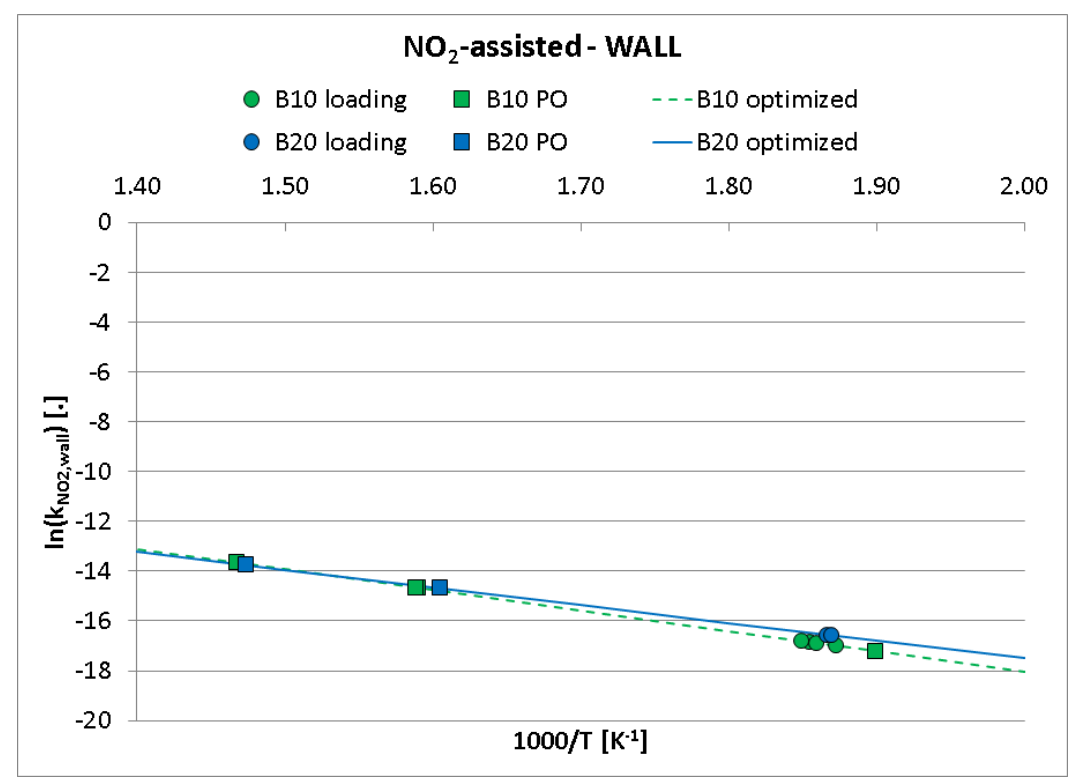

Figure 5.2: Arrhenius plot of $\mathrm{NO}_{2}$-assisted PM oxidation reaction in substrate wall shown for all passive oxidation experiments

Table 5.3: Single set of kinetic parameters of PM oxidation obtained from CPF model calibration

\begin{tabular}{|c|c|c|c|c|c|c|}
\hline Mechanism & Location & Parameter & ULSD & B10 & B20 & Units \\
\hline \multirow{4}{*}{$\mathrm{NO}_{2}$-assisted } & \multirow{2}{*}{ Cake } & $A_{\mathrm{NO}_{2}, \text { cake }}$ & 0.1 & 0.06 & 0.015 & {$[\mathrm{~m} / \mathrm{s}]$} \\
\hline & & $E a_{N_{2}, \text { cake }}$ & 74.1 & 68.3 & 59.4 & [kJ/gmol] \\
\hline & \multirow{2}{*}{ Wall } & $A_{N_{2}, \text { wall }}$ & 0.35 & 0.20 & 0.04 & {$[\mathrm{~m} / \mathrm{s}]$} \\
\hline & & $E a_{\mathrm{NO}_{2}, \text { wall }}$ & 74.1 & 68.3 & 59.4 & [kJ/gmol] \\
\hline \multirow{4}{*}{ Thermal $\left(\mathrm{O}_{2}\right)$} & \multirow{2}{*}{ Cake } & $A_{\text {th,cake }}$ & 0.73 & 0.89 & 1.22 & {$[\mathrm{~m} / \mathrm{s}]$} \\
\hline & & $E a_{\text {th,cake }}$ & \multicolumn{3}{|c|}{139} & [kJ/gmol] \\
\hline & \multirow{2}{*}{ Wall } & $A_{t h, w a l l}$ & 0.58 & 0.74 & 0.99 & {$[\mathrm{~m} / \mathrm{s}]$} \\
\hline & & $E a_{t h, w a l l}$ & \multicolumn{3}{|c|}{139} & [kJ/gmol] \\
\hline
\end{tabular}


simulate the experimental data. Arrhenius plots were then made for all active regeneration experiments and grouped according to fuel-type. Figure 5.3 shows the Arrhenius plot for thermal $\left(\mathrm{O}_{2}\right)$ PM oxidation in the PM cake layer, grouped according to fuel-type (red ULSD, green - B10 and blue - B20). Similarly, Figure 5.4 shows the Arrhenius plot for thermal $\left(\mathrm{O}_{2}\right)$ PM oxidation in the wall. In both figures, the continuous lines show linear fits of the reaction rate constants obtained as a result of a non-linear squares-based optimization performed on the reaction rate constants shown as discrete points on the Arrhenius plot. Also, Table 5.3 shows the corresponding pre-exponential factors and activation energies for thermal $\left(\mathrm{O}_{2}\right)$ oxidation in the PM cake layer and the wall. The activation energy value obtained thus $(139 \mathrm{~kJ} / \mathrm{gmol})$ agrees well with the value for diesel soot as reported in references $[48,49,52,53]$ as shown in Table 2.1 in Section 2.3. Activation energies for thermal oxidation of PM derived from the other fuels (B10 and B20) are not available in the open literature, although reference [49] shows the values obtained for ULSD (129 kJ/gmol) against B100 (160 kJ/gmol) as shown by the Arrhenius plots in Figure 2.7.

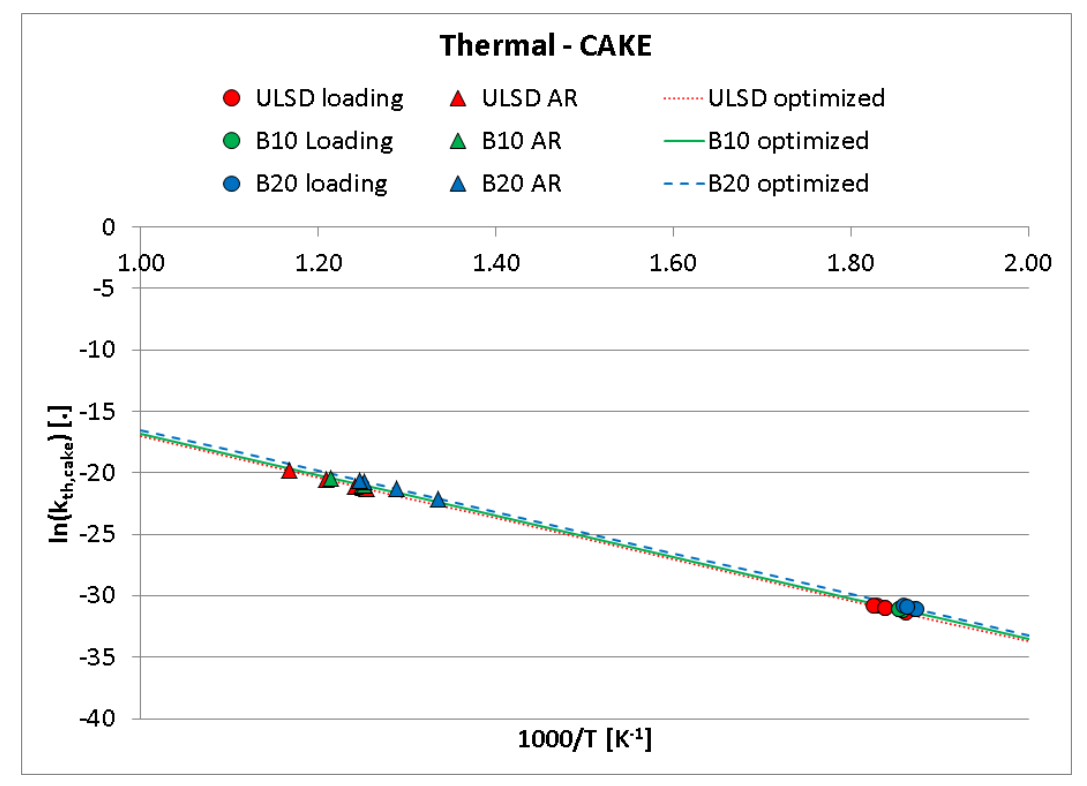

Figure 5.3: Arrhenius plot of thermal $\left(\mathrm{O}_{2}\right)$ PM oxidation reaction in PM cake layer shown for all active regeneration experiments 


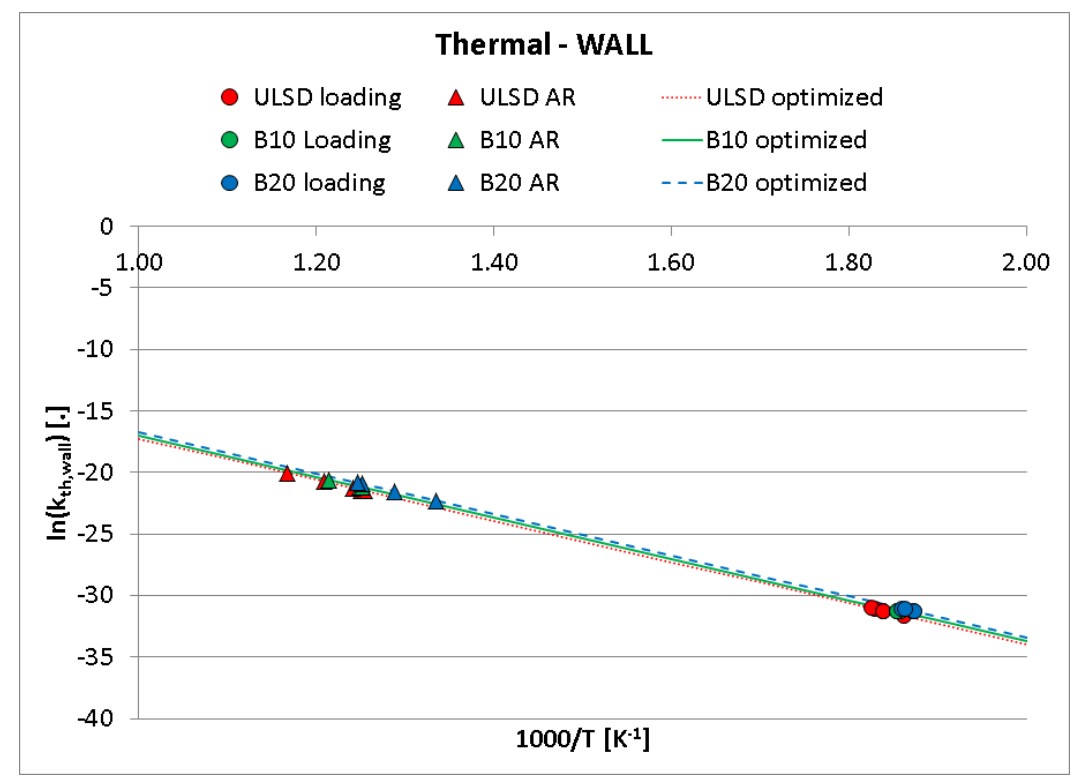

Figure 5.4: Arrhenius plot of thermal $\left(\mathrm{O}_{2}\right)$ PM oxidation reaction in substrate wall shown for all active regeneration experiments 


\subsection{CPF Model Results from Optimized Kinetics}

The model output data compared to the experimental data from two experiments are presented in the chapter - PO-B20-13 and AR-B10-1.

\subsubsection{Passive Oxidation Case}

PO-B20-13 is a passive oxidation experiment carried out at a CPF inlet temperature of $403{ }^{\circ} \mathrm{C}$ and an inlet $\mathrm{NO}_{2} / \mathrm{NO}_{x}$ ratio of 64/204 for a duration of 42 minutes and an inlet $\mathrm{O}_{2}$ concentration of $7.3 \%$ by volume as shown in Table 4.3 . The following sub-sections discuss the relevant results obtained from the model calibration to this experiment using filtration parameters as shown in Table I.1, PM kinetics for fuel type B20 as shown in Table 5.3 and catalytic reaction kinetics as shown in Table 5.2.

\section{Pressure Drop}

The overall pressure drop across the CPF was calibrated to within $(-0.34 /+0.23 \mathrm{kPa})$ of the experimental values of CPF pressure drop measured during the passive oxidation and active regeneration experiments as shown in Table J.1. Figure 5.5 shows the experimental and simulated pressure drop from PO-B20-13. The largest differences observed between experimental and simulated pressure drops were $-0.12 /+0.19 \mathrm{kPa}$. The total simulated $\mathrm{CPF}$ pressure drop is a resultant of 3 different components: cake, wall and channels. These components can be analyzed from the CPF model outputs as shown in Figure 5.5. The highest contribution to the total pressure drop at all points in time for PO-B20-13 is by the substrate wall.

\section{PM Mass}

Figure 5.6 shows the simulated cumulative PM mass balance along with a comparison of experimental (blue circles) and simulated (green line) PM mass retained. A comparison of PM mass retained at the end of various stages for all passive oxidation and active regeneration experiments is shown in Table J.2.

The total PM mass retained is the sum of PM mass retained in the PM cake layer and substrate wall. The results from the calibrated model are as shown in Figure 5.7 for PO-B20-13. The PM mass retained in the PM cake layer accounts for majority of the total PM mass retained in the $\mathrm{CPF}$, whereas the PM mass retained in the substrate wall 


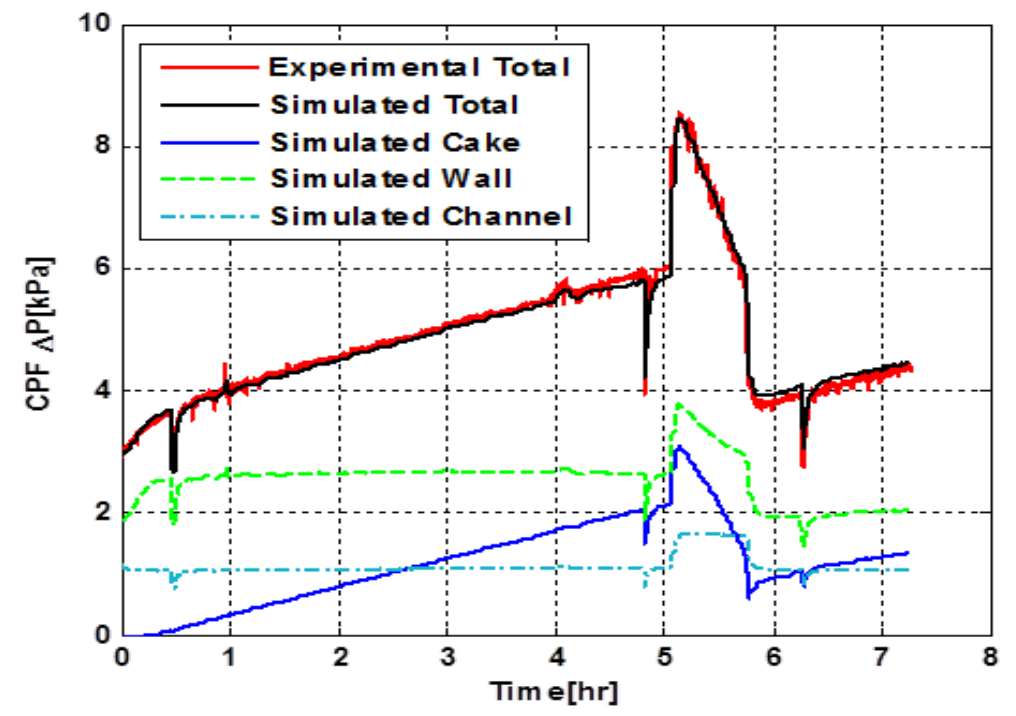

Figure 5.5: Comparison of experimental and model total pressure drop across $\mathrm{CPF}$ and its components - PO-B20-13

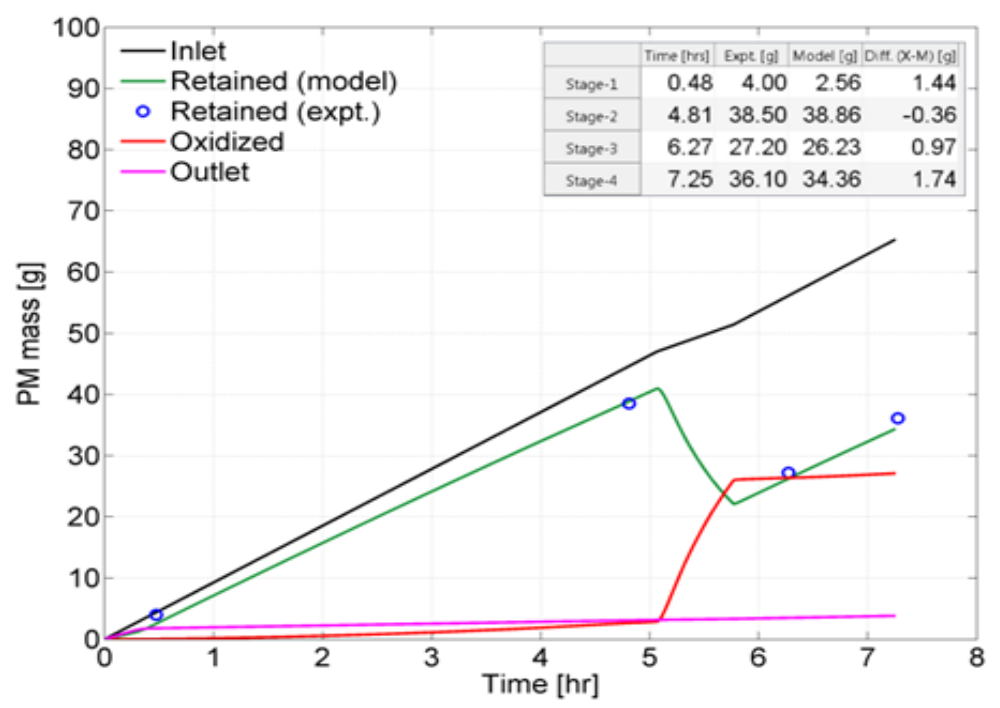

Figure 5.6: Model cumulative PM mass balance and comparison of experimental and model PM mass retained - PO-B20-13 (Table in the inset shows a comparison of experimental and model PM mass retained at the end of all stages) 
increases linearly to $\sim 1.5 \mathrm{~g}$ in the deep bed filtration region (time $<0.3 \mathrm{hrs}$ ) and thereafter decreases to $\sim 0.5 \mathrm{~g}$ during the passive oxidation stage due to the PM oxidation mainly from the $\mathrm{NO}_{2}$-assisted reaction.
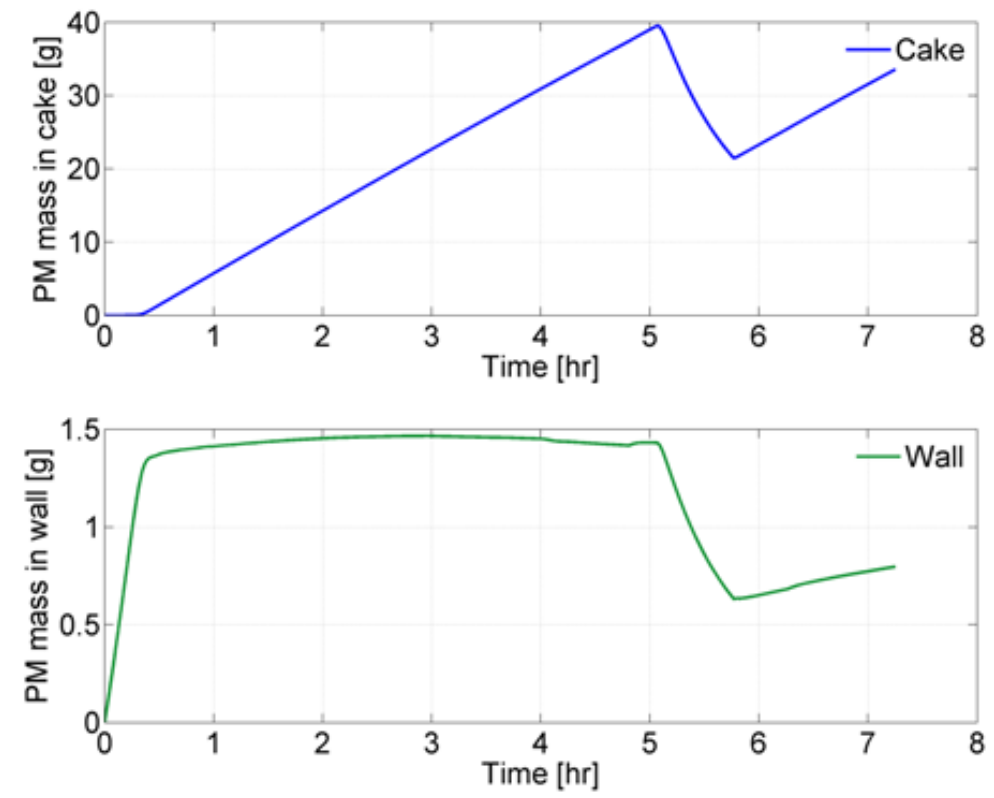

Figure 5.7: Distribution of model total PM mass retained into PM cake and substrate wall PO-B20-13

The PM mass oxidized in the CPF is the sum of that oxidized by two reactions: thermal $\left(\mathrm{O}_{2}\right)$ and $\mathrm{NO}_{2}$-assisted. The calibrated CPF model outputs can be used to derive the distribution of the total PM mass oxidized by thermal and $\mathrm{NO}_{2}$-assisted. A plot of this distribution is shown in Figure 5.8. The top plot shows the instantaneous PM oxidation rates (expressed in $\mathrm{g} / \mathrm{s}$ ) and the bottom plot shows the cumulative PM mass oxidized (expressed in g) through the duration of the experiment. An analysis of the data shows that during this passive oxidation experiment, $95 \%$ of the total PM mass oxidized during passive oxidation (22.7 $\mathrm{g}$ ) was by $\mathrm{NO}_{2}$-assisted PM oxidation reaction.

PM mass oxidized in the filter is also the sum of PM mass oxidized in the PM cake layer and the substrate wall. The outputs from the model can also be used to obtain the distribution of the PM mass oxidized between the PM cake layer and substrate wall as shown in Figure 5.9. This shows that the majority of PM mass oxidation occurs in the PM cake layer which was $96 \%$ of the total PM mass oxidized during the passive oxidation experiment. 

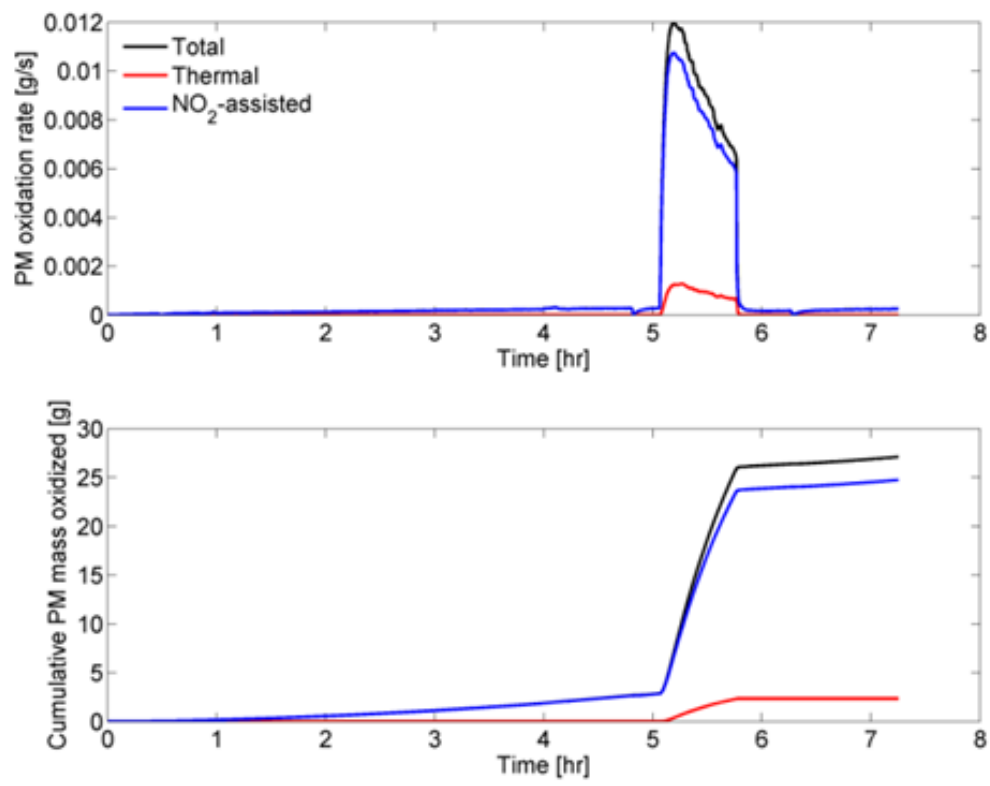

Figure 5.8: Distribution of total PM oxidation rate (top) and cumulative PM mass oxidized (bottom) into thermal (red) and $\mathrm{NO}_{2}$-assisted (blue) mechanisms
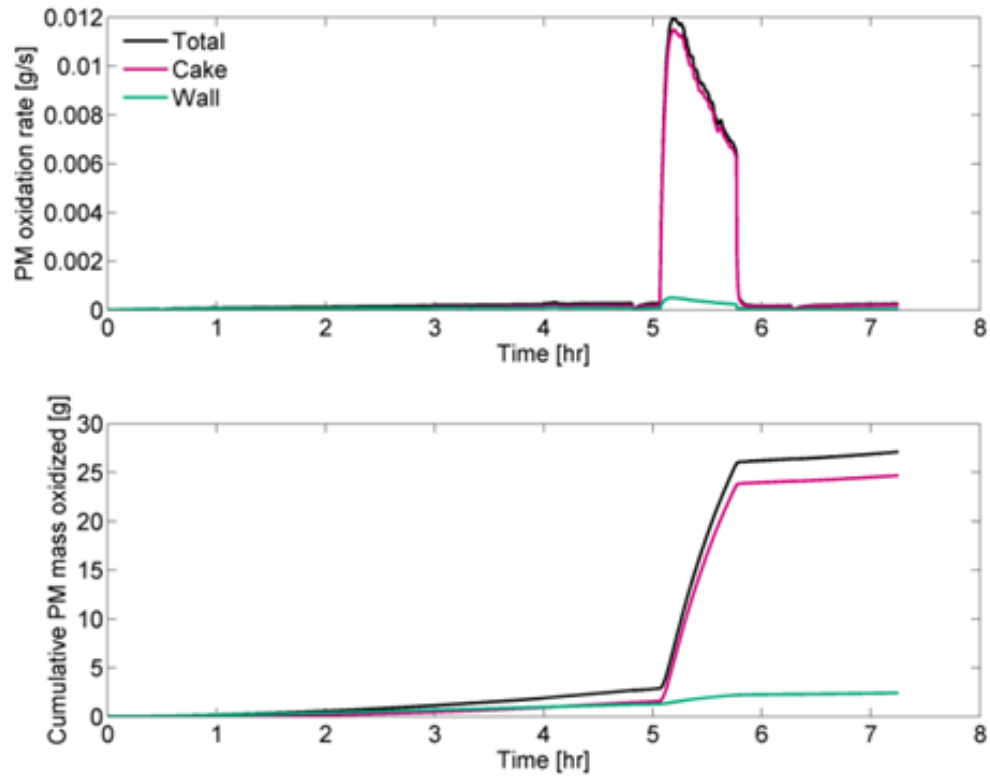

Figure 5.9: Distribution of total PM oxidation rate (top) and cumulative PM mass oxidized (bottom) into PM cake (purple) and substrate wall (green) - PO-B20-13 


\section{Filtration Efficiency}

Figure 5.10 shows the simulated total filtration efficiency of the CPF compared to the PM mass-based filtration efficiency measured ( $97 \%$ - shown as the black ' $x$ ') during stage-2 loading. This plot also shows the components of the efficiency namely cake and wall. The total filtration efficiency of the filter at initiation of the experiments comes entirely from the substrate wall (37\%). Filtration efficiency of the substrate wall increases to about $47 \%$ due to wall PM loading during deep-bed filtration, followed by PM cake layer formation. Once PM cake layer starts to form, cake filtration efficiency increases to $95.8 \%$ and wall filtration efficiency remains constant at $\sim 47 \%$.

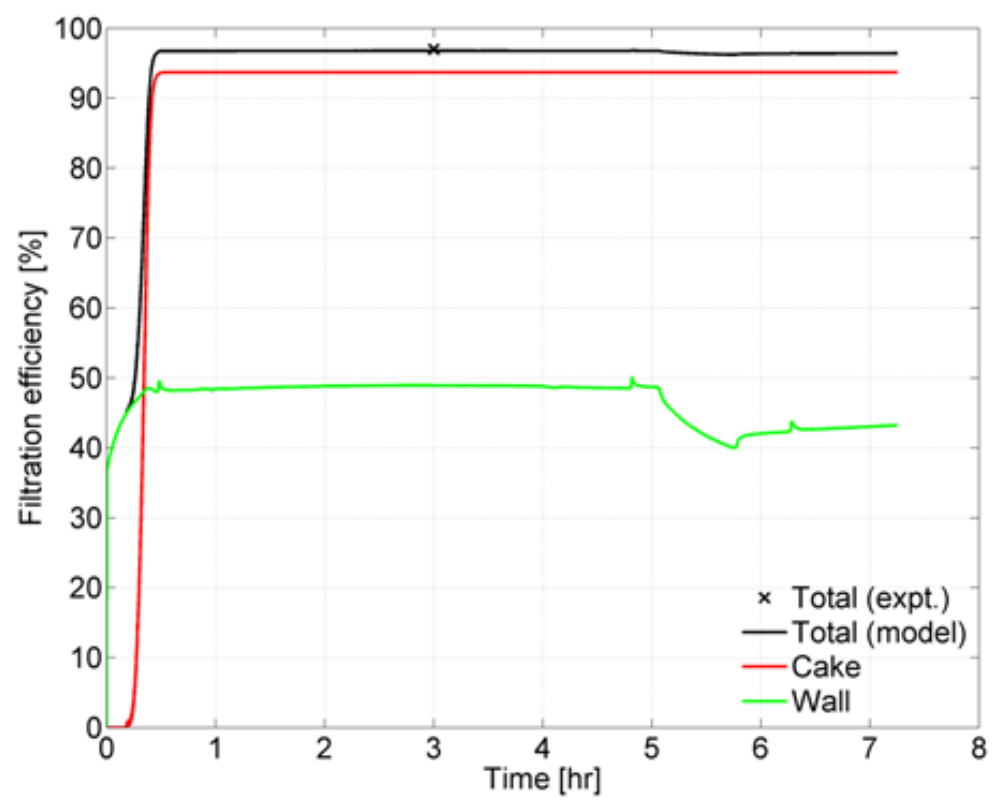

Figure 5.10: Comparison of experimental and model total filtration efficiency and distribution of filtration efficiency into PM cake and substrate wall - PO-B20-13

\section{Exhaust Gas Temperature}

The CPF model calculates the exhaust gas temperature as it passes through the inlet channel, substrate wall and outlet channel. Agreement with experimentally measured temperature data is critical in accurately predicting the rates of all reactions since they are strongly dependent on the temperature via the exponential term. Figure 5.11 shows a comparison of simulated and experimental CPF outlet temperatures for PO-B20-13, showing agreement within $-2 /+1{ }^{\circ} \mathrm{C}$ of the experimental outlet temperature values as well as transient behavior (thermal response) of the CPF. 


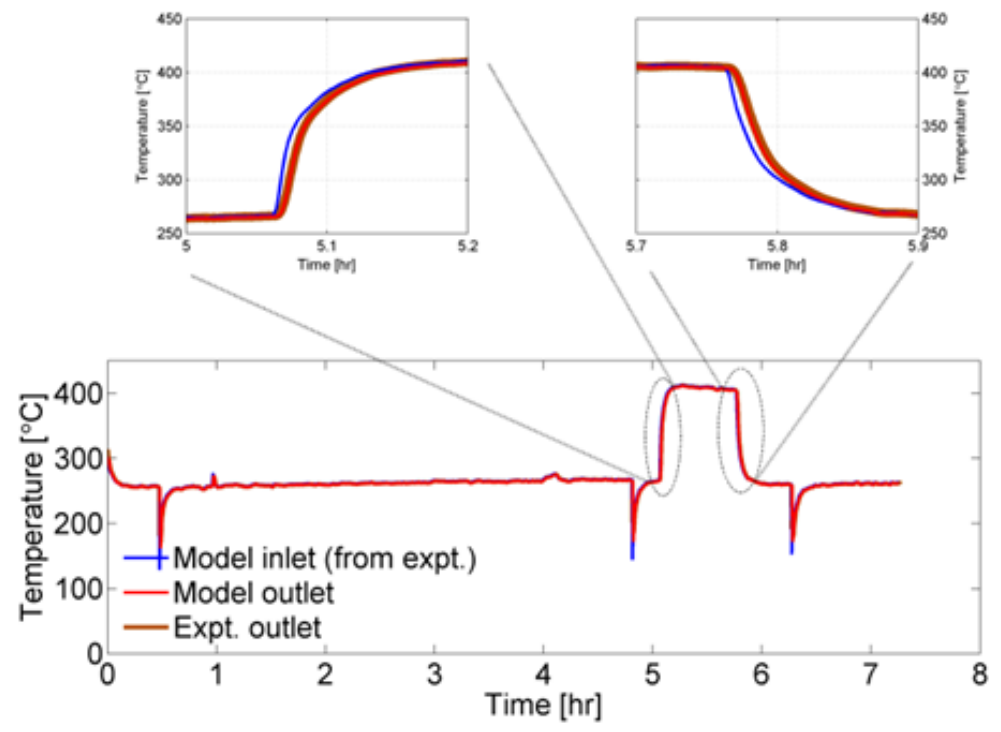

Figure 5.11: Comparison of experimental and model CPF outlet gas temperature with model input CPF inlet gas temperature - PO-B20-13 [Top left: comparison of temperatures for 12 minutes during the start of passive oxidation stage, Top right: comparison of temperatures for 12 minutes during the end of passive oxidation stage, Bottom: comparison of temperatures for the entire duration of the experiment] 


\section{$\mathrm{NO}_{2}$ Concentration}

Figure 5.12 shows CPF inlet $\mathrm{NO}_{2}$ concentrations from measured experimental values as well as a comparison of model outlet $\mathrm{NO}_{2}$ concentrations (red line) to experimental CPF outlet $\mathrm{NO}_{2}$ concentrations (red 'x'), showing that the overall agreement of model $\mathrm{NO}_{2}$ concentrations was within $-15 /+12$ ppm of experimental values for PO-B20-13.

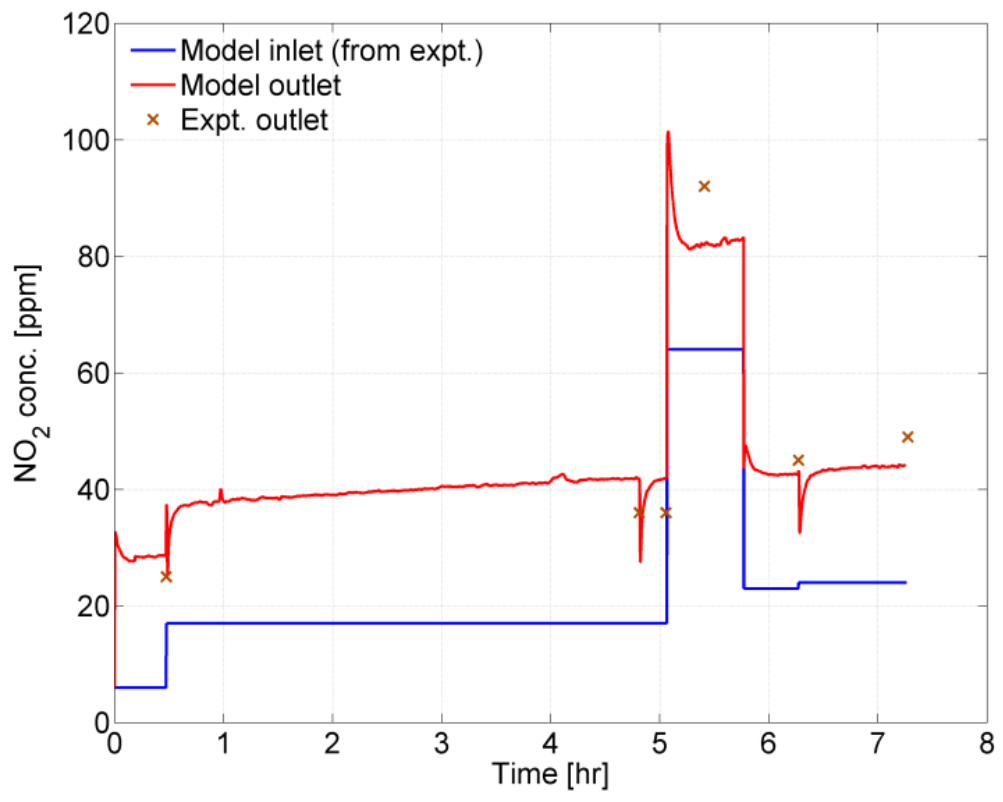

Figure 5.12: Comparison of experimental and model CPF outlet $\mathrm{NO}_{2}$ concentrations with model input CPF inlet $\mathrm{NO}_{2}$ concentrations - PO-B20-13

\subsubsection{Active Regeneration Case}

AR-B10-1 is an active regeneration experiment carried out at a CPF inlet temperature of $530{ }^{\circ} \mathrm{C}$ and $\mathrm{CPF}$ inlet $\mathrm{NO}_{2} / \mathrm{NO}_{x}$ ratio of 4/119 for a duration of 26 minutes and CPF inlet $\mathrm{O}_{2}$ concentration of $7.8 \%$ by volume as shown in Table 4.4 . The following sub-sections discuss the relevant results obtained from the model calibration to this experiment using filtration parameters as shown in Table I.1, PM kinetics for fuel type B20 as shown in Table 5.3 and catalytic reaction kinetics as shown in Table 5.2. 


\section{Pressure Drop}

A comparison of experimental and simulated CPF pressure drop and its components, namely cake, wall and channels for AR-B10-1 is shown in Figure 5.13. The maximum difference between experimental and simulated pressure drop in this experiment was 0.3 $\mathrm{kPa}$. As was noted in the passive oxidation test case, wall pressure drop dominated the total pressure drop at all points in time during this experiment also.

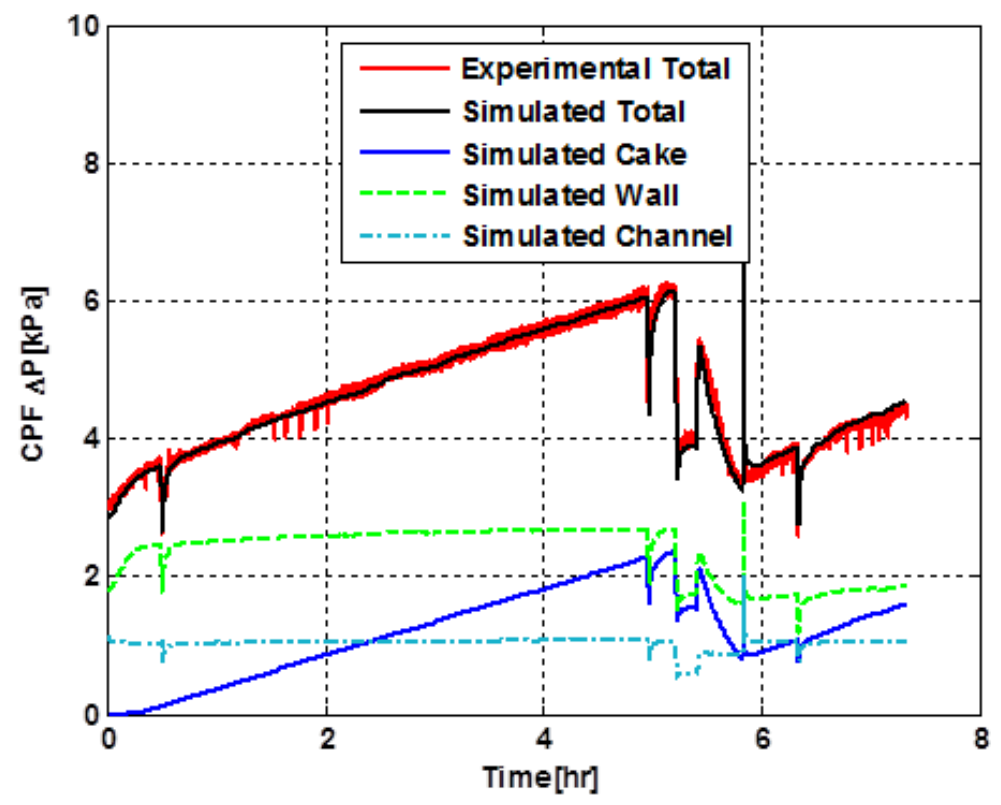

Figure 5.13: Comparison of experimental and model total pressure drop across $\mathrm{CPF}$ and its components - AR-B10-1

PM Mass

A cumulative PM mass balance simulated by the model is shown in Figure 5.14. The PM mass retained in this experiment was calibrated to within $(-1.21 /+0.90 \mathrm{~g})$ of experimental values.

Figure 5.15 shows the distribution of PM mass retained in the PM cake layer and substrate wall, showing that the PM retained in the PM cake layer accounts for most of the total PM retained at all points in the simulation. The wall PM mass at the end of loading stage- 2 was $1.49 \mathrm{~g}$, which reduced to $0.33 \mathrm{~g}$ at the end of active regeneration.

Figure 5.16 shows the distribution of PM mass oxidation rate (top) and cumulative PM mass oxidized (bottom) by thermal $\left(\mathrm{O}_{2}\right)$ and $\mathrm{NO}_{2}$-assisted PM oxidation reactions, 


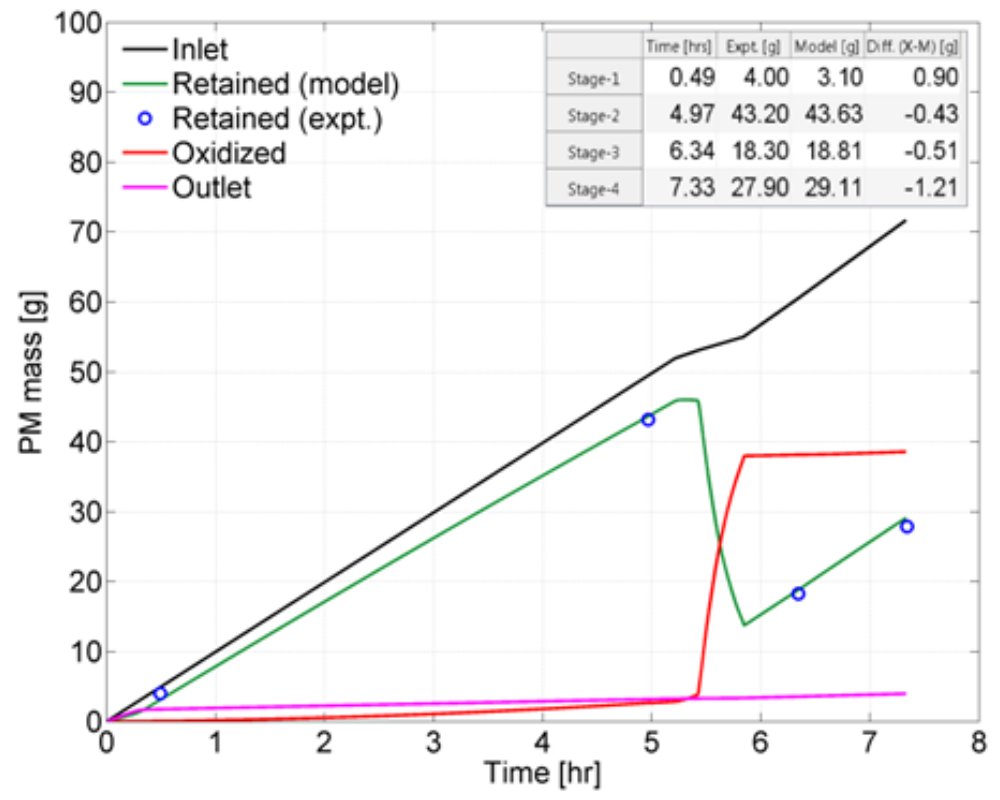

Figure 5.14: Model cumulative PM mass balance and comparison of experimental and model PM mass retained - AR-B10-1 (Table in the inset shows a comparison of experimental and model PM mass retained at the end of all stages)
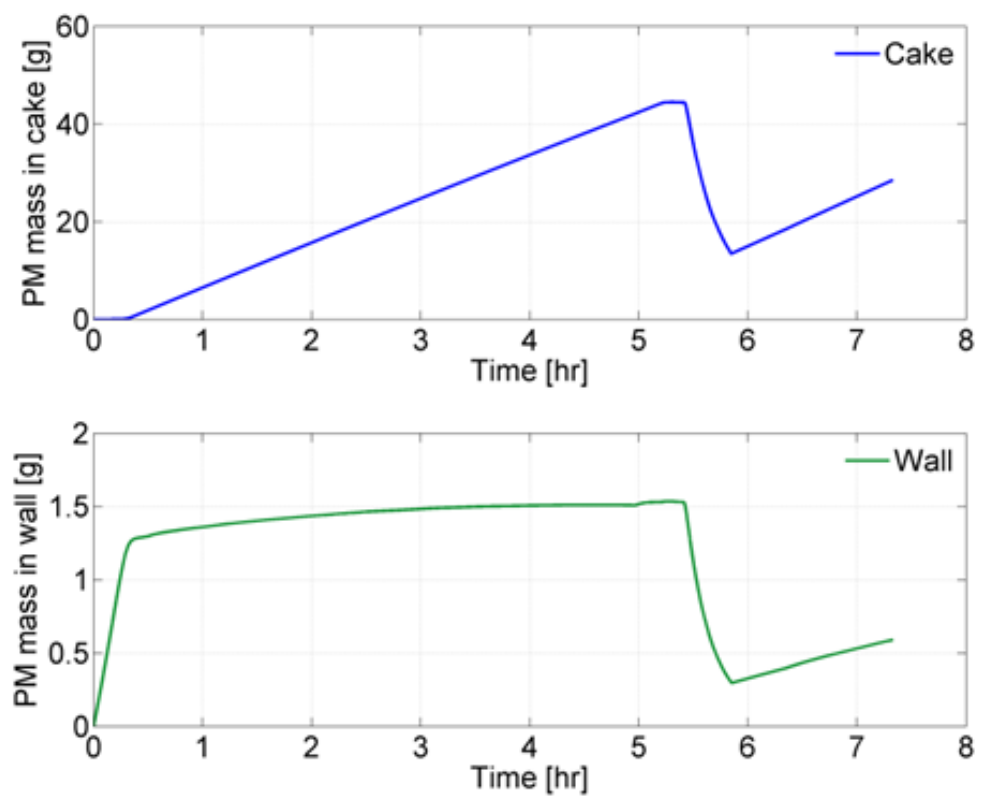

Figure 5.15: Distribution of model total PM mass retained into PM cake and substrate wall AR-B10-1 
showing that the distribution of percentage PM mass oxidized during the active regeneration stage for AR-B10-1 is $83.5 \%$ (thermal) - $16.5 \%\left(\mathrm{NO}_{2}\right.$-assisted).
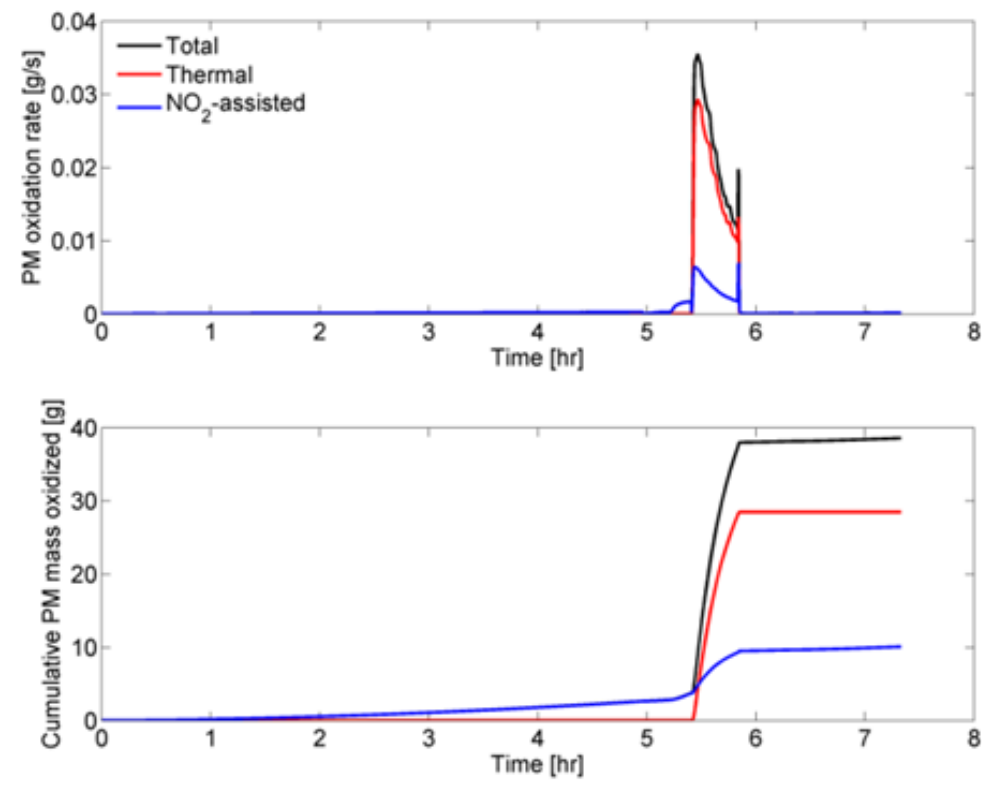

Figure 5.16: Distribution of total PM oxidation rate (top) and total cumulative PM mass oxidized (bottom) into thermal (red) and $\mathrm{NO}_{2}$-assisted (blue) mechanisms - AR-B10-1

Figure 5.17 shows the distribution of PM mass oxidation rate (top) as well as cumulative PM mass oxidized (bottom) in the PM cake layer and substrate wall. The majority of PM mass oxidized during regeneration is in the PM cake layer (96.2\%).

\section{Filtration Efficiency}

Figure 5.18 shows a comparison of experimental and simulated CPF total filtration efficiency as well as the components of the filtration efficiency for the cake and wall, showing the initiation of filtration occurring in the substrate wall and subsequent formation of PM cake layer, as well as agreement between stage- 2 overall filtration efficiency (black line) and experimental filtration efficiency measured (black ' $x$ ') within $(0.1 \%)$.

\section{Exhaust Gas Temperature}

Figure 5.19 shows a comparison of experimental and simulated CPF outlet temperatures showing agreement within $-5 /+7{ }^{\circ} \mathrm{C}$ of the simulated outlet temperature with the 

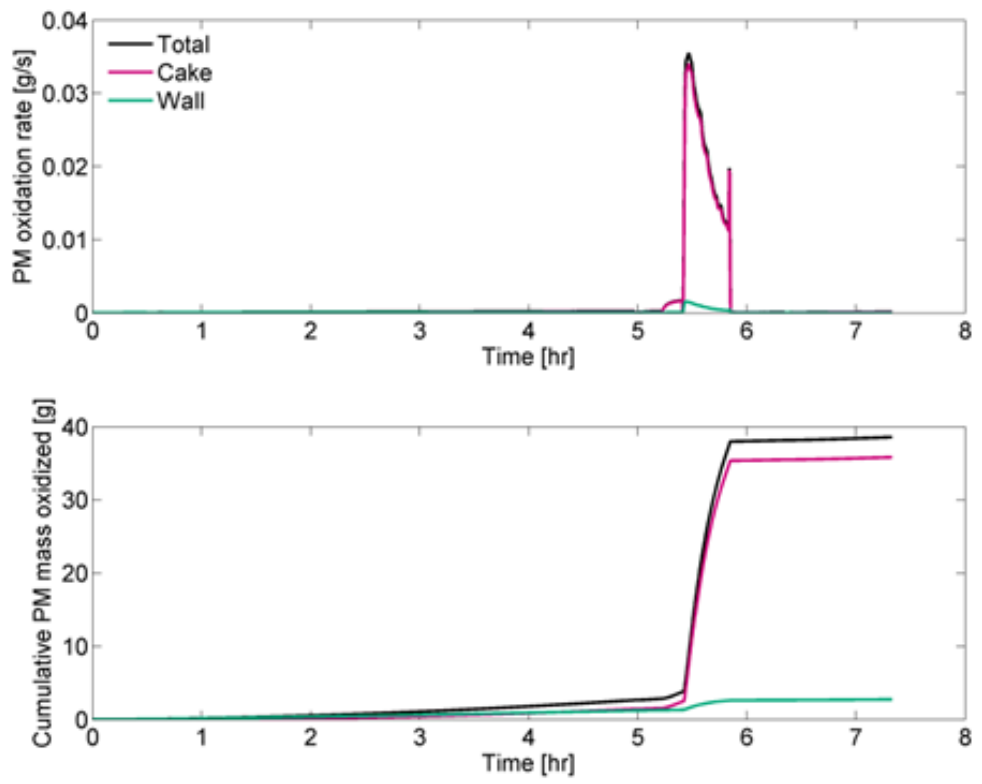

Figure 5.17: Distribution of total PM oxidation rate (top) and total cumulative PM mass oxidized (bottom) into PM cake (purple) and substrate wall (green) - AR-B10-1

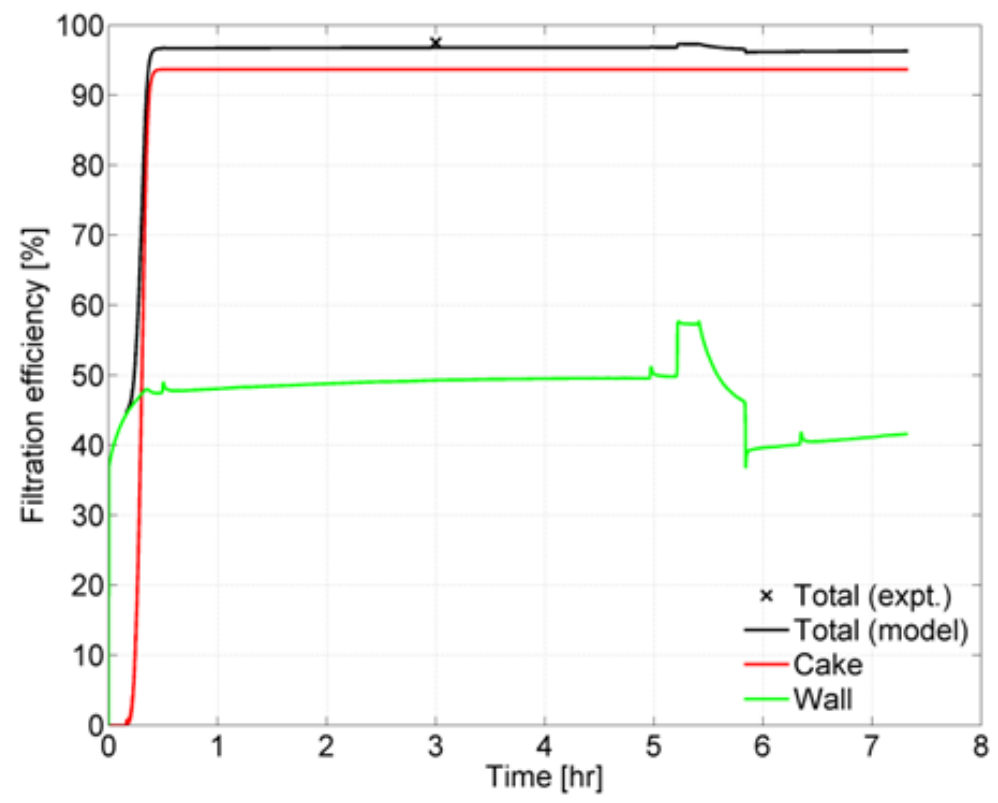

Figure 5.18: Comparison of experimental and model total filtration efficiency and distribution of filtration efficiency into filtration efficiency of PM cake and filtration efficiency of substrate wall AR-B10-1 
experimentally measured average outlet temperature in value as well as transient response.

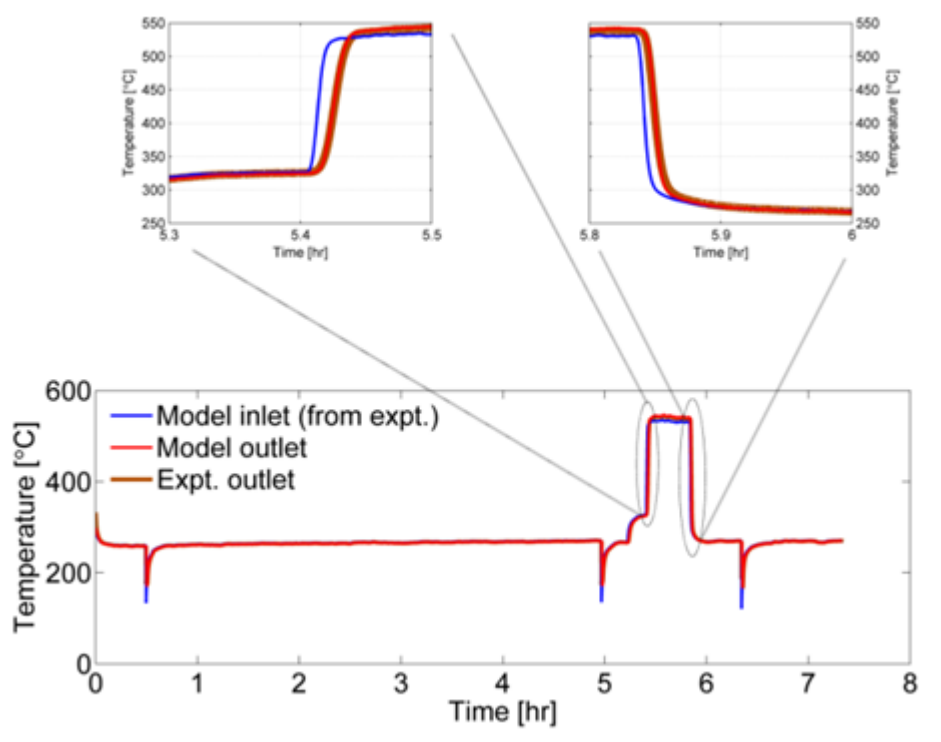

Figure 5.19: Comparison of experimental and model CPF outlet gas temperature with model input CPF inlet gas temperature - AR-B10-1

\section{$\mathrm{NO}_{2}$ Concentration}

Figure 5.20 shows the $\mathrm{CPF}$ inlet $\mathrm{NO}_{2}$ concentrations as well as a comparison of model CPF outlet $\mathrm{NO}_{2}$ concentrations (red line) to experimentally measured CPF outlet $\mathrm{NO}_{2}$ concentrations ( $\mathrm{red}$ ' $\mathrm{x}$ '), showing agreement within $-13 /+14 \mathrm{ppm}$ of model values to experimental data. 


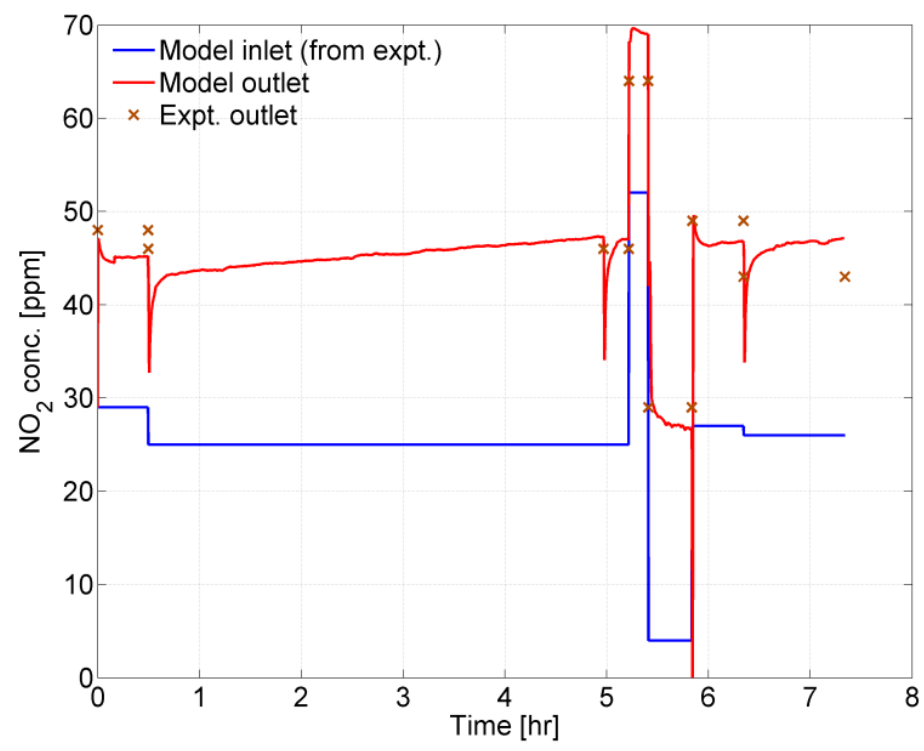

Figure 5.20: Comparison of experimental and model CPF outlet $\mathrm{NO}_{2}$ concentrations with model input CPF inlet $\mathrm{NO}_{2}$ concentrations - AR-B10-1 


\subsection{Analysis of PM Mass Oxidized by Mechanism}

An analysis of the CPF model outputs was carried out to understand trends in the fraction of total PM mass oxidized by mechanism. For this analysis, the total PM mass oxidized and its components during the passive oxidation phase of all passive oxidation experiments and during the active regeneration phase of all active regeneration experiments were determined. The result of this analysis is as shown in Figure 5.21.

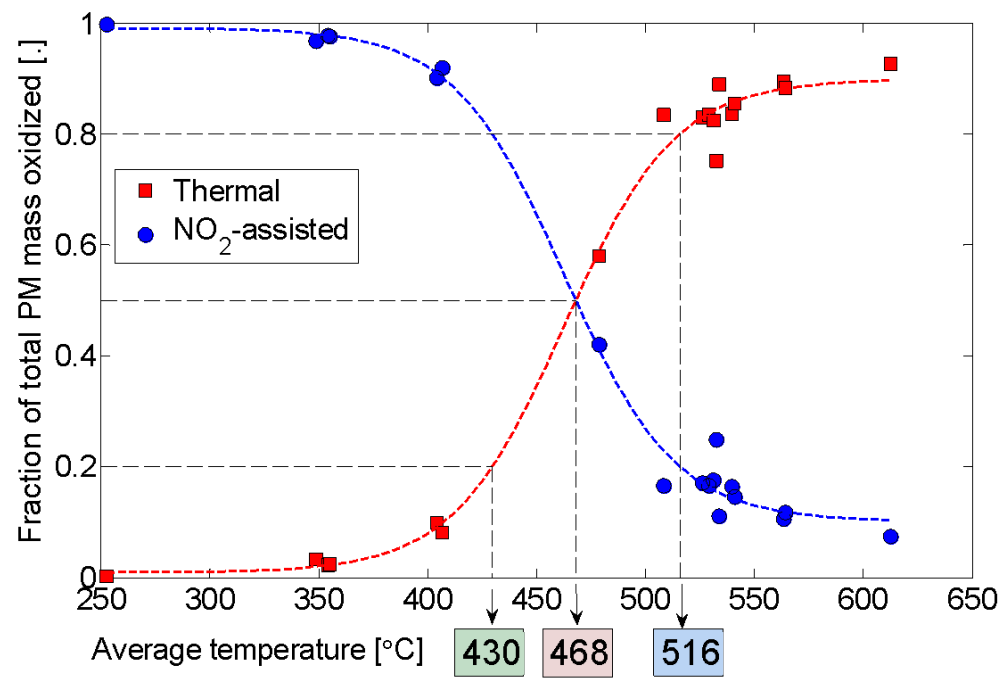

Figure 5.21: Fraction of total PM mass oxidized by thermal and $\mathrm{NO}_{2}$-assisted PM oxidation reactions plotted against average $\mathrm{CPF}$ temperature during passive oxidation and active regeneration stages of all experiments considered for CPF model calibration

Figure 5.21 shows that the PM oxidation at lower temperatures (which also have significant levels of CPF inlet $\mathrm{NO}_{2}$ concentrations - 61-112 ppm for all passive oxidation cases considered in this study) is dominated by $\mathrm{NO}_{2}$-assisted PM oxidation. At higher temperatures and lower $\mathrm{CPF}$ inlet $\mathrm{NO}_{2}$ concentrations $(3-10 \mathrm{ppm}$ for all active regeneration cases considered in this study), thermal PM oxidation dominates the oxidation of PM. Shown in Figure 5.21 are also three temperatures where the fraction of thermal to total PM mass oxidized increases from 0.2 to 0.8 . The Figure 5.21 also shows that at a temperature of $430^{\circ} \mathrm{C}, 20 \%$ of the total PM mass oxidized is by the thermal PM oxidation reaction. At $468^{\circ} \mathrm{C}$, this fraction becomes $50 \%$ and at $516^{\circ} \mathrm{C}, 80 \%$ of the total $\mathrm{PM}$ mass oxidized is by the thermal PM oxidation reaction. The highest fractional PM mass oxidized by thermal PM oxidation reaction is $\sim 0.9$ for 4 of the 12 active regeneration cases. Further studies can be carried out by parametrically varying $\mathrm{NO}_{2}$ concentrations and temperature for realistic engine operating conditions to determine the trend of $\mathrm{NO}_{2}$-assisted and thermal 
$\left(\mathrm{O}_{2}\right)$ PM oxidation. Also, after obtaining the calibrated PM oxidation kinetic parameters, an analysis of the thermal to total and $\mathrm{NO}_{2}$-assisted to total reaction rates for the three different fuels was carried out as shown in Figure K.1 in Appendix K. The major difference between data presented in Figure 5.21 and results shown in K.1 is that the data in 5.21 shows the combined effect of two differences:

1. Figure 5.21 shows results from all three fuels (out of the eighteen total experiments, five were ULSD, seven were B10 and 6 were B20), and

2. For the data in Figure 5.21, CPF inlet $\mathrm{NO}_{2}$ concentrations for each experiment were different from that used in calculations of $\mathrm{NO}_{2}$-assisted reaction rate towards results presented in Figure Figure K.1 due to the thermodynamic equilibrium between $N O$ and $\mathrm{NO}_{2}$ taking place in the DOC (for instance, these were 61-112 ppm for PO experiments (Table 4.3) where $\mathrm{CPF}$ inlet temperatures were in the $253-408^{\circ} \mathrm{C}$ range and 3-10 ppm for all AR experiments (Table 4.4) where the CPF temperatures were in the $476-581^{\circ} \mathrm{C}$ range) than the constant $\mathrm{NO}_{2}$ concentration $(=50 \mathrm{ppm})$ used for Figure K.1.

\subsection{Analysis of Reaction Rates Obtained from Calibrated PM Oxidation Kinetic Parameters}

The total reaction rate for PM oxidation as a function of $\mathrm{NO}_{2}$ concentration and temperature can be evaluated using the PM kinetics obtained from model calibration. Figure 5.22 shows a plot of thermal $\left(\mathrm{O}_{2}=9 \%\right.$ by volume $)$ and $\mathrm{NO}_{2}$-assisted reaction rates for several $\mathrm{NO}_{2}$ concentrations for ULSD. The corresponding plots for B10 and B20 are shown in Figures 5.23 and 5.24 respectively. Figure 5.22 shows that thermal PM oxidation reaction rate is equal to $\mathrm{NO}_{2}$-assisted $\mathrm{PM}$ reaction rate at $440^{\circ} \mathrm{C}$ for $10 \mathrm{ppm} \mathrm{NO}_{2}$. The $\mathrm{NO}_{2}$-assisted PM reaction rates increase as the $\mathrm{NO}_{2}$ concentrations increase from 50-300 $\mathrm{ppm}$ and the thermal and $\mathrm{NO}_{2}$-assisted $\mathrm{PM}$ reaction rates are equal at $600^{\circ} \mathrm{C}$ for $100 \mathrm{ppm}$ $\mathrm{NO}_{2}$.

Figure 5.25 shows a comparison of $\mathrm{NO}_{2}$-assisted, thermal $\left(\mathrm{O}_{2}=9 \%\right.$ Vol. $)$ and total reaction rates as functions of increasing temperatures for 3 different $\mathrm{NO}_{2}$ concentrations: $\mathrm{NO}_{2}=10 \mathrm{ppm}$ (top), $\mathrm{NO}_{2}=50 \mathrm{ppm}$ (middle) and $\mathrm{NO}_{2}=150 \mathrm{ppm}$ (bottom) for ULSD fuel-type, showing that:

1. As temperature increases, given a specific $\mathrm{NO}_{2}$ concentration and fuel-type, the fractions of total reaction rate from thermal $\left(\mathrm{O}_{2}\right)$ oxidation increase, and

2. In the temperature range of $350-550^{\circ} \mathrm{C}$, more than $50 \%$ of the total reaction rate is 


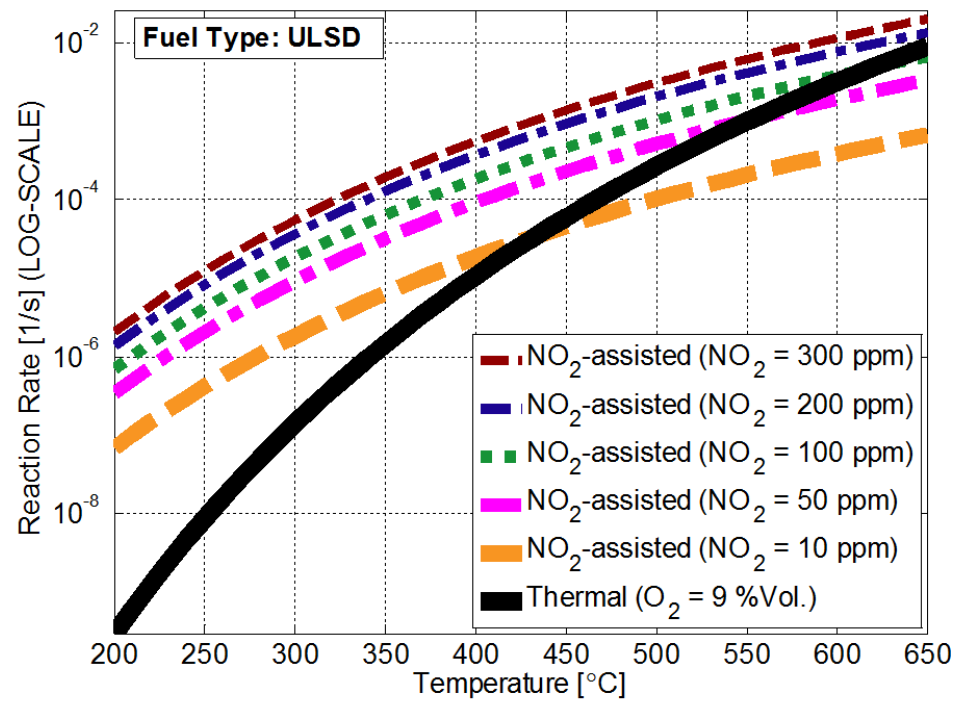

Figure 5.22: $\mathrm{PM}$ oxidation reaction rates (plotted in log-scale) showing $\mathrm{NO}_{2}$-assisted reaction rates at 5 different $\mathrm{NO}_{2}$ concentrations with thermal $\left(\mathrm{O}_{2}\right)$ reaction (black line) rate shown as functions of temperature for ULSD fuel-type

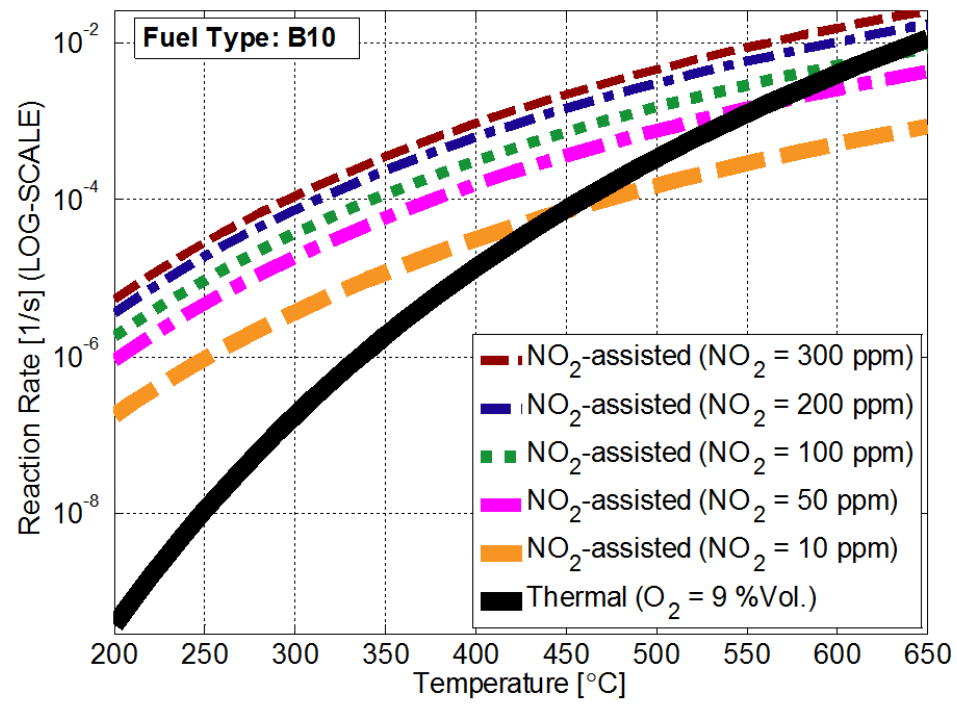

Figure 5.23: PM oxidation reaction rates (plotted in log-scale) showing $\mathrm{NO}_{2}$-assisted reaction rates at 5 different $\mathrm{NO}_{2}$ concentrations with thermal $\left(\mathrm{O}_{2}\right)$ reaction (black line) rate shown as functions of temperature for B10 fuel-type 


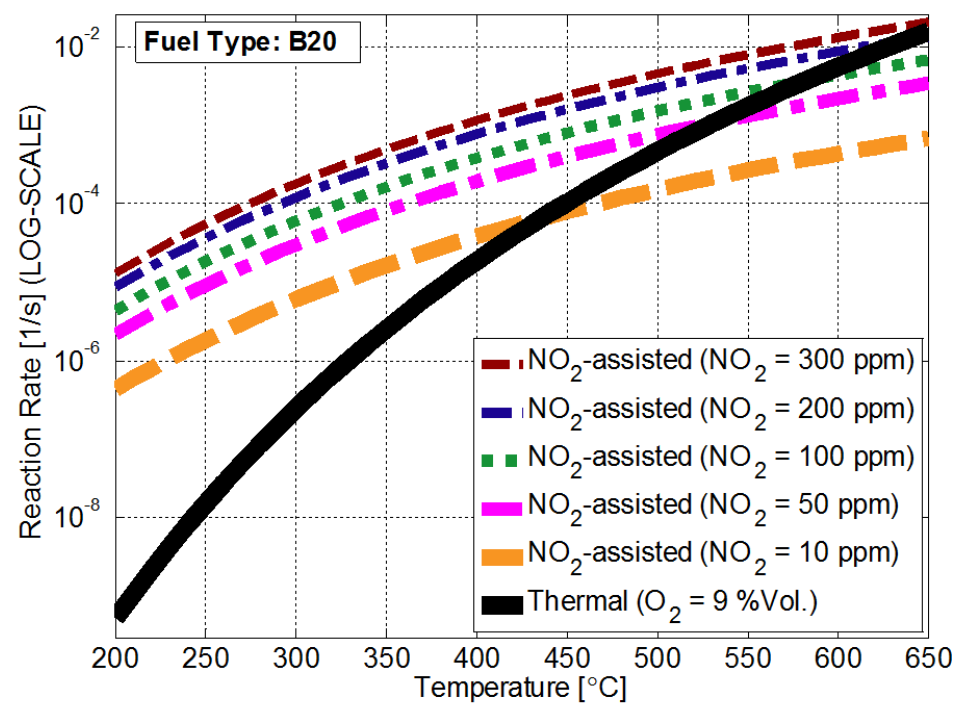

Figure 5.24: PM oxidation reaction rates (plotted in log-scale) showing $\mathrm{NO}_{2}$-assisted reaction rates at 5 different $\mathrm{NO}_{2}$ concentrations with thermal $\left(\mathrm{O}_{2}\right)$ reaction (black line) rate shown as functions of temperature for B20 fuel-type 
due to $\mathrm{NO}_{2}$-assisted reaction if the $\mathrm{NO}_{2}$ concentrations are greater than $50 \mathrm{ppm}$ (middle and bottom plots of Figure 5.25). For $\mathrm{NO}_{2}=10 \mathrm{ppm}$ (top plot of Figure 5.25), the temperature at which $\mathrm{NO}_{2}$-assisted and thermal $\left(\mathrm{O}_{2}\right)$ reaction rates are equal is $445^{\circ} \mathrm{C}$.

Corresponding comparisons of $\mathrm{NO}_{2}$-assisted, thermal $\left(\mathrm{O}_{2}=9 \%\right.$ Vol. $)$ and total reaction rates as functions of increasing temperatures for fuel-types B10 and B20 are shown in Figures 5.26 and 5.27 respectively. Also, Table K.1 in Appendix K shows the total reaction rates obtained from the calibrated PM oxidation kinetics (as shown in Table 5.3) for the three fuels presented for one $\mathrm{O}_{2}$ concentration $=9 \%$ by volume and three different $\mathrm{NO}_{2}$ concentrations $=50,100$ and $150 \mathrm{ppm}$ respectively. In addition, Figure K.2 shows the comparison of total PM reaction rates for the three fuels (ULSD (red line), B10 (green line) and B20 (blue line)) showing that the total PM reaction rates are higher for increasing biodiesel content. This agrees with trends observed from experimental data about total reaction rates obtained for different fuel types (ULSD, B10 and B20) in references $[4,61]$ and comparisons of ULSD and B100 fuels carried out in reference [49] as shown in Figure 2.7 in Chapter 2.

The contour plot in Figure 5.28 shows the lines of equal total reaction rates (green lines) as functions of $\mathrm{NO}_{2}$ concentrations and temperatures obtained from the calibrated PM kinetics for ULSD fuel-type. Also shown in the plot are lines of equal ratio of $\mathrm{NO}_{2}$-assisted to total PM oxidation reaction rate (blue lines). As an illustration, one of the active regeneration experiments is shown in Figure 5.28 with a red ' $x$ '. This was an active regeneration condition conducted at a $\mathrm{CPF}$ inlet $\mathrm{NO}_{2}$ concentration of $7 \mathrm{ppm}$ and $581^{\circ} \mathrm{C} \mathrm{CPF}$ inlet temperature and had a total reaction rate $=2.3 \times 10^{-3} \mathrm{~s}^{-1}$. The magenta dashed line shows the line connecting all $\mathrm{NO}_{2}$ concentration - temperature combinations that have the same total reaction rate $\left(=2.3 \times 10^{-3} \mathrm{~s}^{-1}\right)$. Hence the same reaction rate can be obtained by operating the $\mathrm{CPF}$ at lower temperatures and higher $\mathrm{CPF}$ inlet $\mathrm{NO}_{2}$ concentrations (for example, $\mathrm{NO}_{2}=200 \mathrm{ppm}$, temperature $=499^{\circ} \mathrm{C} \& \mathrm{NO}_{2}=300 \mathrm{ppm}$, temperature $=477^{\circ} \mathrm{C}$ ). Similar plots for fuel-types B10 and B20 are shown in Figures 5.29 and 5.30 respectively. 


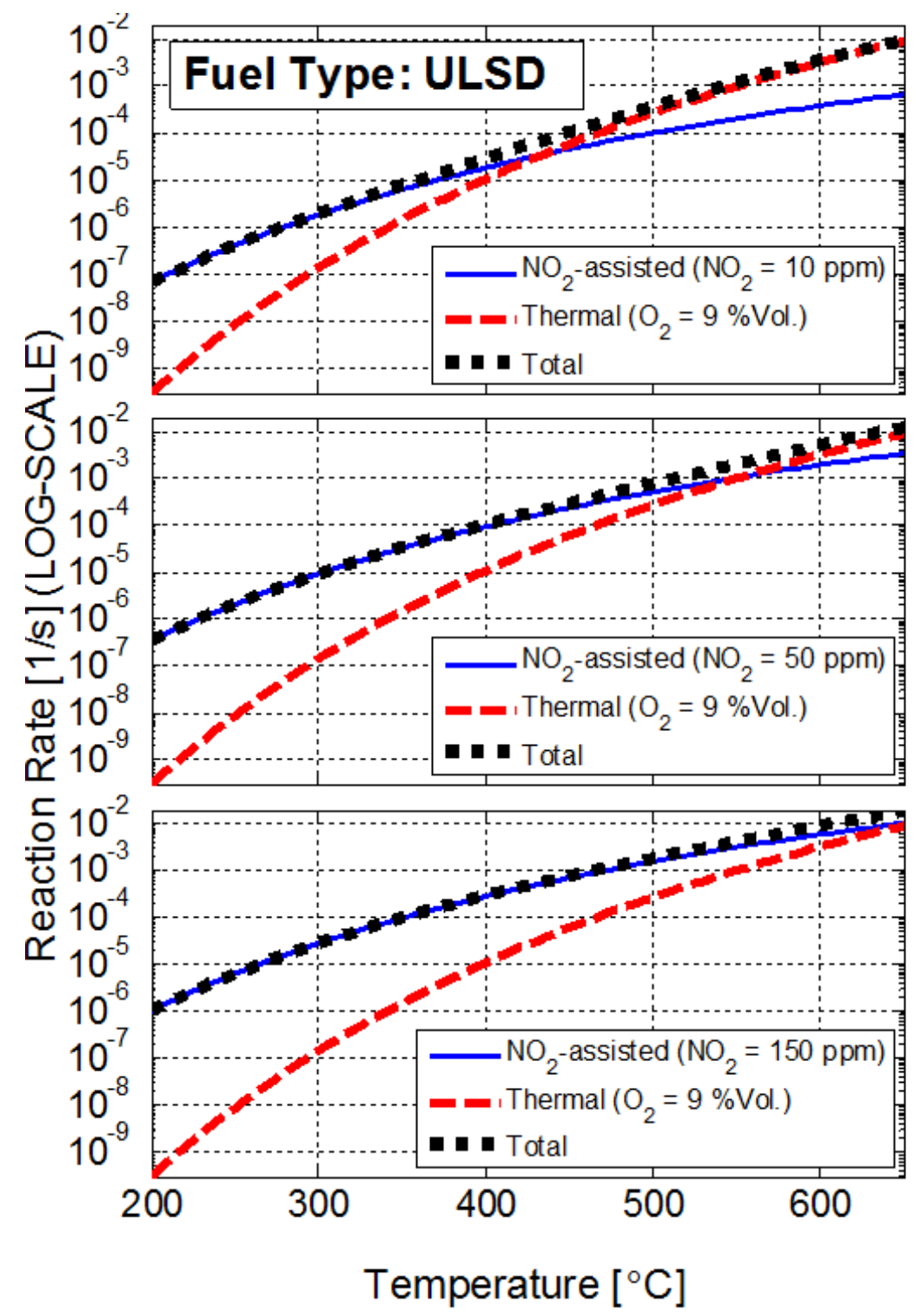

Figure 5.25: Comparison of $\mathrm{NO}_{2}$-assisted (blue), thermal (red) and total (black) shown for 3 different $\mathrm{NO}_{2}$ concentrations (top $=10 \mathrm{ppm}$, middle $=50 \mathrm{ppm}$ and bottom $=150 \mathrm{ppm}$ ) as a function of increasing temperature for ULSD fuel-type 


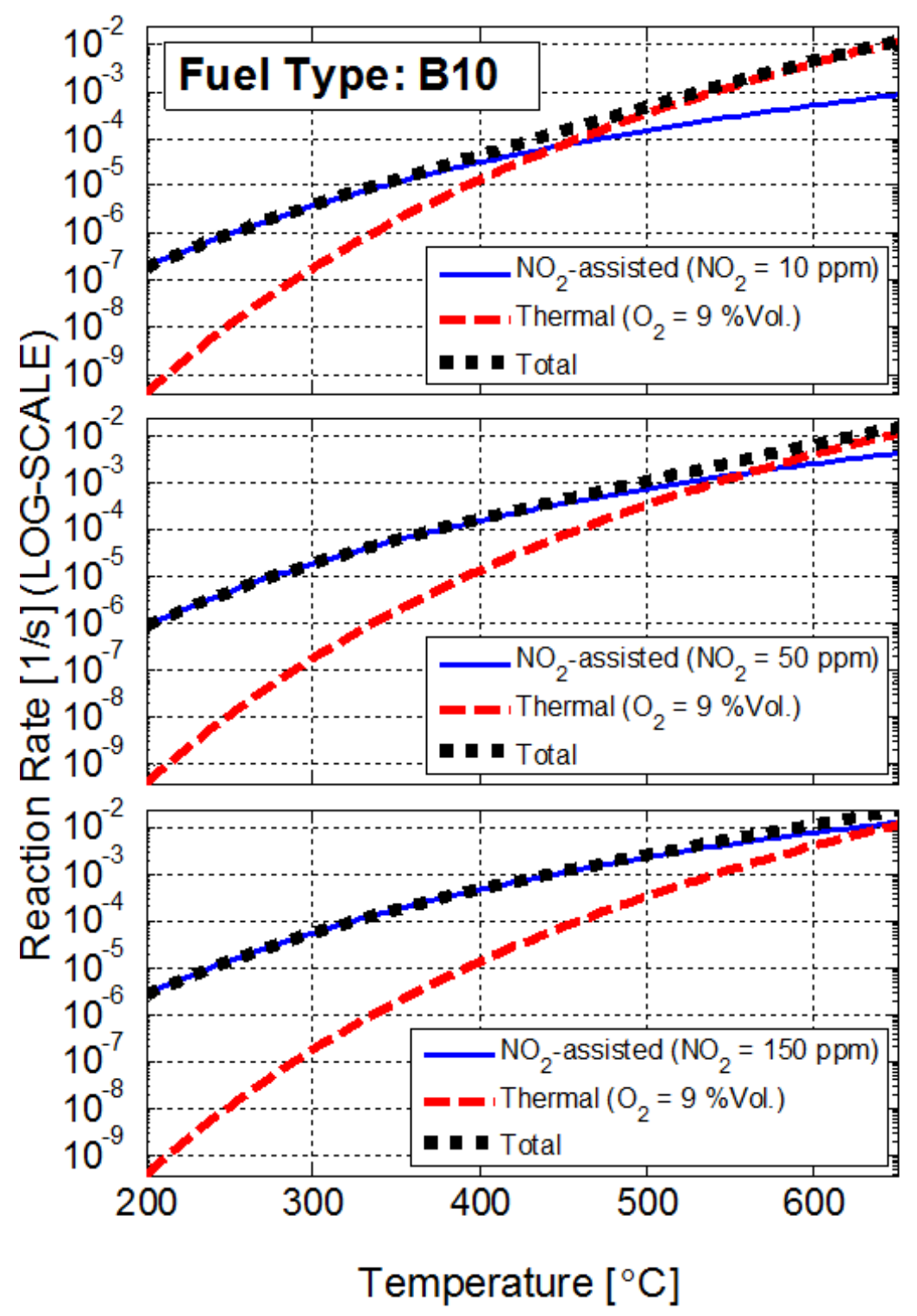

Figure 5.26: Comparison of $\mathrm{NO}_{2}$-assisted (blue), thermal (red) and total (black) shown for 3 different $\mathrm{NO}_{2}$ concentrations (top $=10 \mathrm{ppm}$, middle $=50 \mathrm{ppm}$ and bottom $=150 \mathrm{ppm}$ ) as a function of increasing temperature for B10 fuel-type 


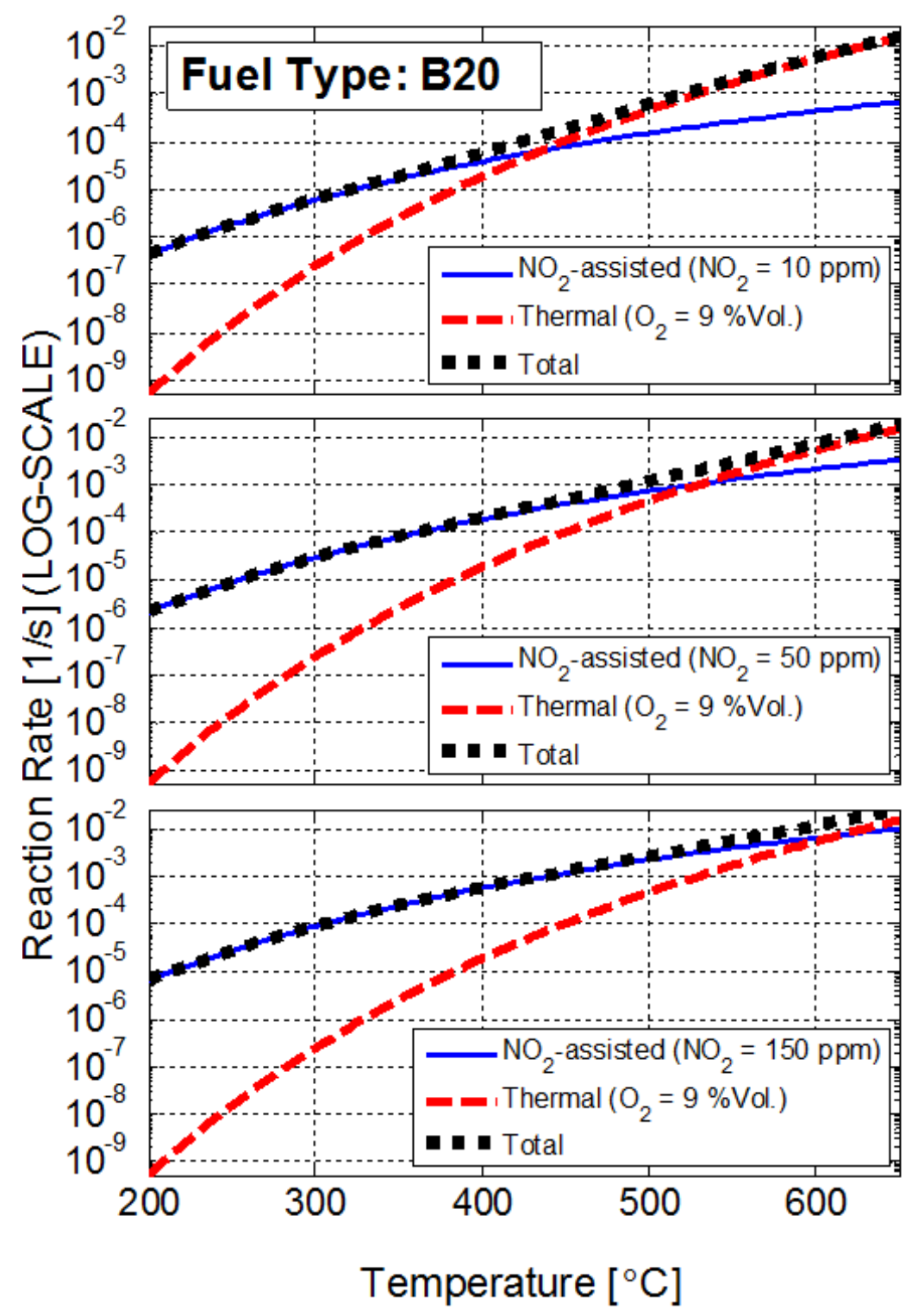

Figure 5.27: Comparison of $\mathrm{NO}_{2}$-assisted (blue), thermal (red) and total (black) shown for 3 different $\mathrm{NO}_{2}$ concentrations (top $=10 \mathrm{ppm}$, middle $=50 \mathrm{ppm}$ and bottom $=150 \mathrm{ppm}$ ) as a function of increasing temperature for B20 fuel-type 


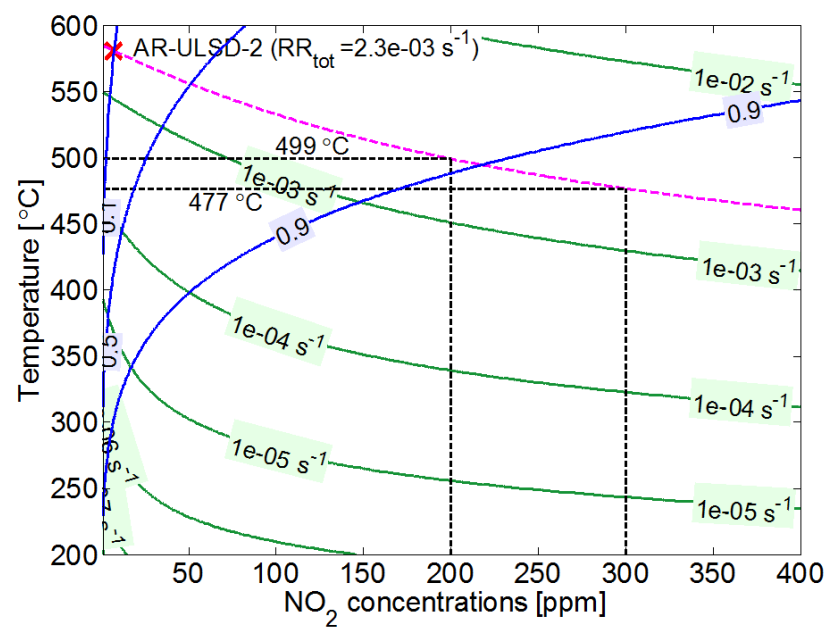

Figure 5.28: Plot showing lines of equal total reaction rates (green) from calibrated PM kinetics obtained and lines of equal fraction of $\mathrm{NO}_{2}$-assisted to total PM oxidation reaction rate (blue) for the ULSD fuel type as functions of increasing $\mathrm{NO}_{2}$ concentrations along $\mathrm{x}$ axis and increasing temperatures along $y$ axis. The red ' $\mathrm{x}$ ' shows one active regeneration experiment (AR-ULSD-2: $N O_{2}=7 \mathrm{ppm}$, Temp. $=581^{\circ} \mathrm{C}$, total reaction rate from calibrated model $\left.=2.3 \times 10^{-03} \mathrm{~s}^{-1}\right)$

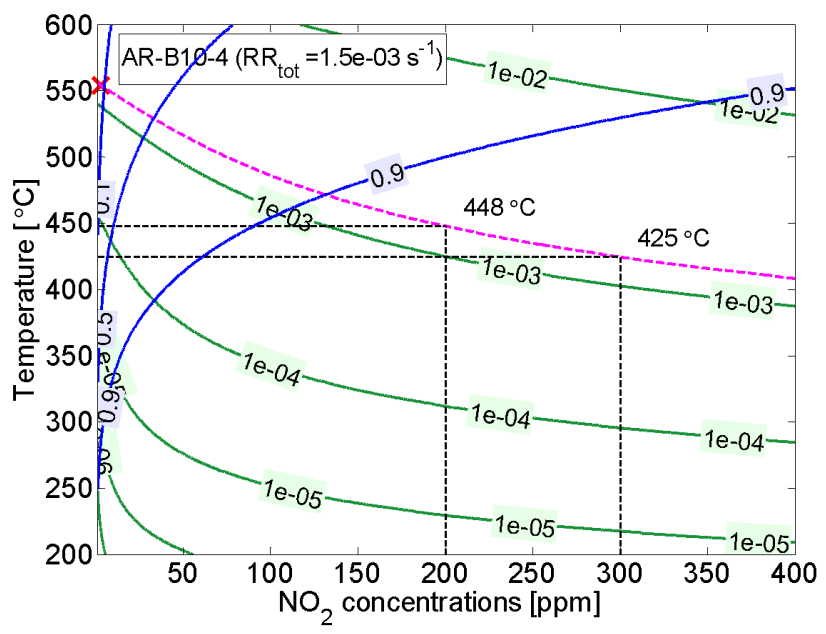

Figure 5.29: Plot showing lines of equal total reaction rates (green) from calibrated PM kinetics obtained and lines of equal fraction of $\mathrm{NO}_{2}$-assisted to total PM oxidation reaction rate (blue) for the $\mathrm{B} 10$ fuel type as functions of increasing $\mathrm{NO}_{2}$ concentrations along $\mathrm{x}$ axis and increasing temperatures along $y$ axis. The red ' $x$ ' shows one active regeneration experiment (AR-B10-4: $\mathrm{NO}_{2}=3 \mathrm{ppm}$, Temp. $=554{ }^{\circ} \mathrm{C}$, total reaction rate from calibrated model $=1.5 \times 10^{-03} \mathrm{~s}^{-1}$ ) 


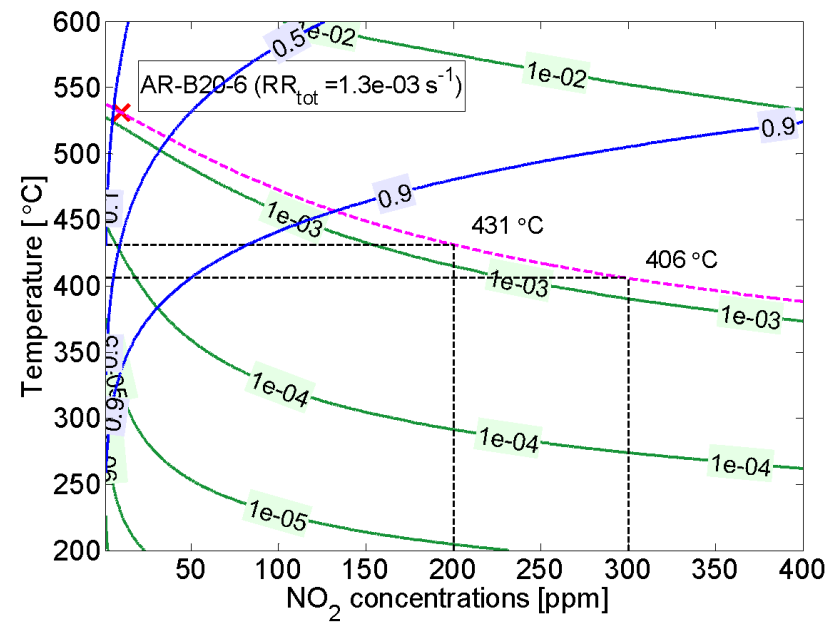

Figure 5.30: Plot showing lines of equal total reaction rates (green) from calibrated PM kinetics obtained and lines of equal fraction of $\mathrm{NO}_{2}$-assisted to total PM oxidation reaction rate (blue) for the B20 fuel type as functions of increasing $\mathrm{NO}_{2}$ concentrations along $\mathrm{x}$ axis and increasing temperatures along $y$ axis. The red ' $x$ ' shows one active regeneration experiment (AR-B20-6: $N O_{2}=10 \mathrm{ppm}$, Temp. $=531{ }^{\circ} \mathrm{C}$, total reaction rate from calibrated model $=1.3 \times 10^{-03} \mathrm{~s}^{-1}$ ) 


\subsection{Effect of PM Oxidation on PM Cake Layer Thickness}

In the CPF model, PM flow rate is assumed to be proportional to the local wall-flow velocity as described in section 3.4.6. Wall-flow velocity at initiation of experiment is always lowest in the middle; highest at outlet end. Accordingly, PM cake layer thickness at end of loading for both PO and AR test cases show similar profiles (Figure 5.31 for passive oxidation and Figure 5.32 for active regeneration). This is also consistent with trends observed by other recent research work that studied the axial distribution of PM at the end of loading via PM imaging techniques [74]. A comparison of experimental data that is described in reference [39] to model result at similar loading points is discussed later in this section.

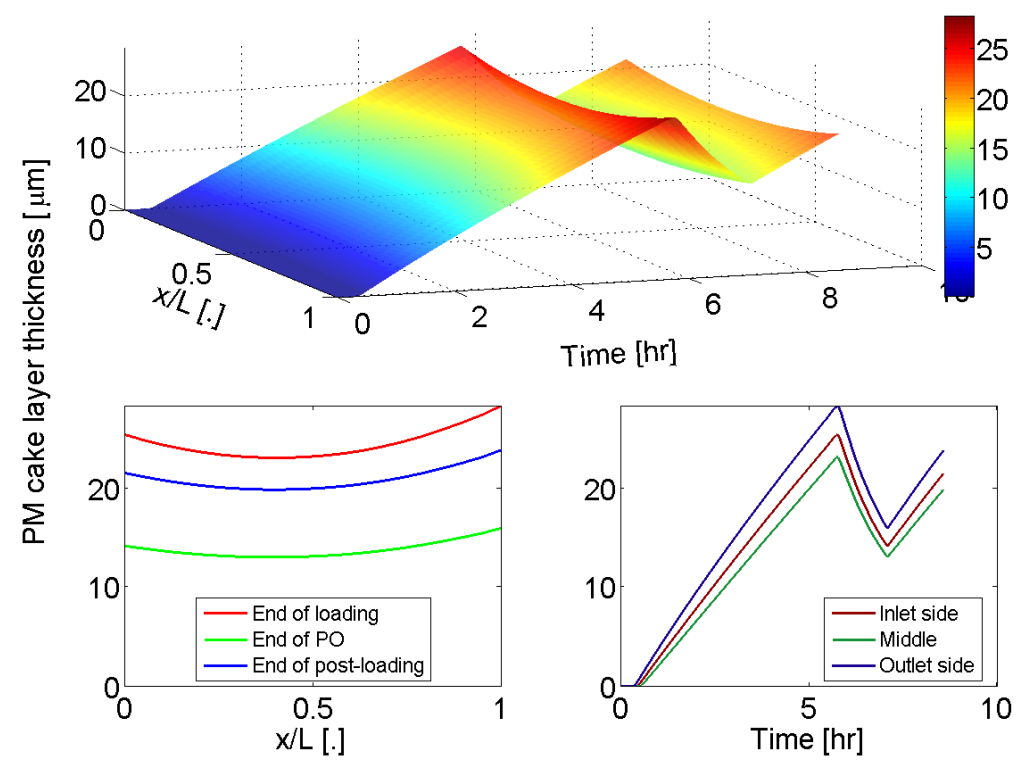

Figure 5.31: Plots showing the variation of PM cake layer thickness along axial (x) direction and time, with smaller plots showing: (lower left) PM cake thickness profiles at 3 different time points and (lower right) the cake thickness at 3 different axial locations - PO-B20-12

At the end of passive oxidation, as shown in Figure 5.31, oxidation rate of PM in the cake layer is equal axially due to moderate reaction rates $\left(\mathrm{RR}=2.9 \times 10^{-4} \mathrm{~s}^{-1}\right)$ of $\mathrm{PM}$ through the $\mathrm{NO}_{2}$-assisted mechanism. However, at the end of active regeneration (Figure 5.32), cake PM oxidation at the outlet end is more than at the inlet end because thermal $\left(\mathrm{O}_{2}\right)$ oxidation is dependent on temperature (outlet end was $\sim 15^{\circ} \mathrm{C}$ higher than the inlet end due to energy release occurring due to $H C$ oxidation). The total reaction rate at the end of active regeneration for AR-B10-1 was $8.87 \times 10^{-4} \mathrm{~s}^{-1}$ ).

Recent research at MTU $[39,74]$ has shown that the axial PM distribution for a CPF 


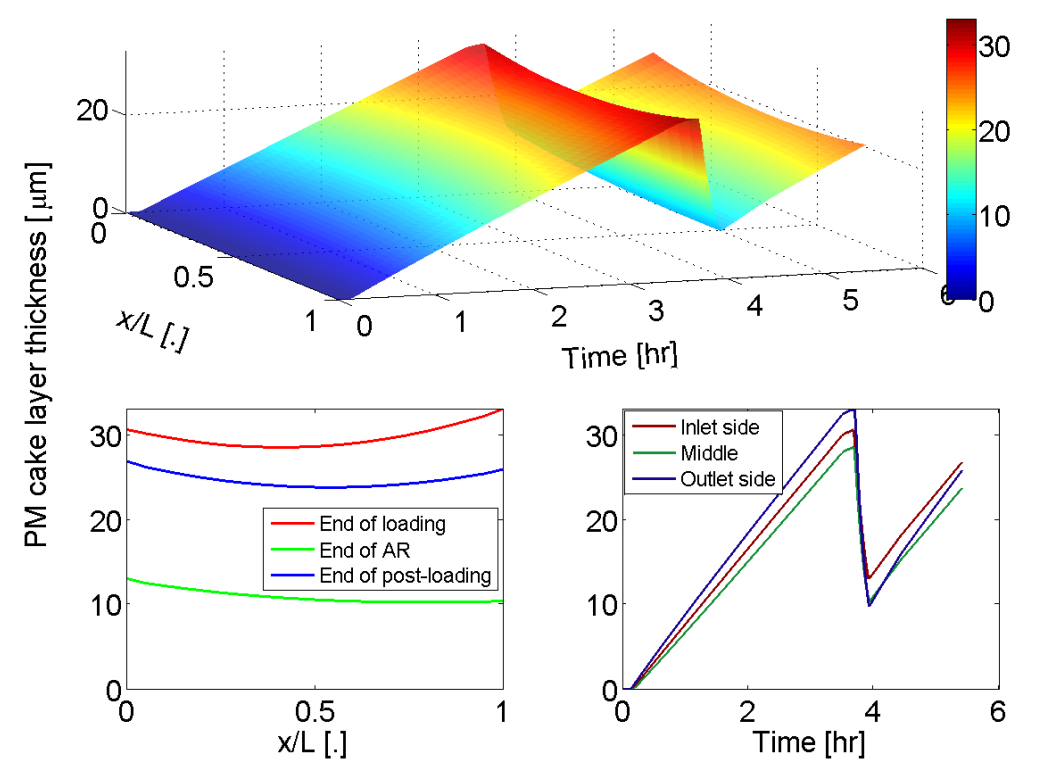

Figure 5.32: Plots showing the variation of PM cake layer thickness along axial (x) direction and time, with smaller plots showing: (lower left) the cake thickness profiles at 3 different time points and (lower right) the cake thickness at 3 different axial locations - AR-ULSD-1

loaded with PM from clean state is not uniform. This was thought to be the case when the CPF model was being developed as is shown by the red and green lines in Figure 5.33. Since PM is carried into the filter by the exhaust gas mixture, the rate at which PM has to be carried into each axial location in the CPF needs to be directly proportional to the local exhaust gas volumetric flow rate in accordance with conservation of PM mass. Hence, in the CPF model, the PM inlet rate is calculated as being directly proportional to the local wall-flow velocity as described in Chapter 3. Figure 5.33 also shows the axial PM loading distribution result (blue line) from the CPF model corresponding to a total PM mass retained in the filter of $46.5 \mathrm{~g}(2.72 \mathrm{~g} / \mathrm{l})$. The loading in $\mathrm{g} / \mathrm{l}$ is in turn calculated from the PM cake layer thickness model output. The non-uniform growth of PM cake layer seen from the model output is due to the model improvement in the axial dependency of PM flow rate. The other observations that can be made here are:

- The experimental data show the loading distribution at two overall PM loadings (3.08 $\mathrm{g} / \mathrm{l}$ and $2.81 \mathrm{~g} / \mathrm{l})$. The profiles in each case were similar in trend, i.e., the inlet end $(\mathrm{x} / \mathrm{L}=0)$ and the outlet end $(\mathrm{x} / \mathrm{L}=1)$ had higher PM deposition rates compared to the middle $(\mathrm{x} / \mathrm{L}=0.5)$.

- The model output shows the solution of the growth of the PM cake layer from clean state which assumes no axial migration of PM in the PM cake layer. This is most 


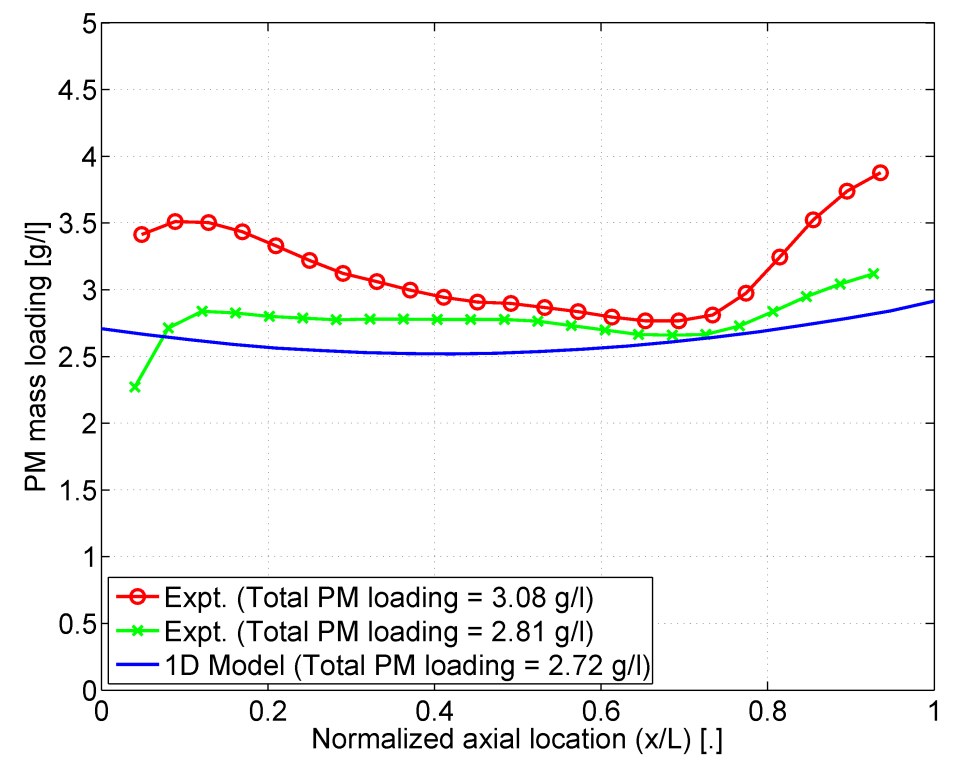

Figure 5.33: Axial PM loading distribution from two sets of experimental data [39] compared to a similar point in the simulation of AR-B10-1 at the end of loading stage-2

likely the reason behind the plugged outlet end of the $\mathrm{CPF}(\mathrm{x} / \mathrm{L}=0.8$ to 0.95$)$ showing a larger variation in PM loading in the both sets of experimental data compared to the model output.

- Experimental data towards both inlet and outlet ends $(x / L<0.05$ and $x / L>0.95)$ were not considered reliable and hence have been eliminated from the plots but have to be accounted for when calculating the total PM loading levels.

Also, similar qualitative agreement of trends can be seen between the experimental PM distribution data [39] and CPF model outputs as shown in Figure 5.34 at the end of passive oxidation and in Figure 5.35 at the end of active regeneration. In the experimental data shown in Figure 5.35, the experimental data included both PM and ash where the ash loading was estimated to be $\sim 0.9 \mathrm{~g} / \mathrm{l}$, therefore the presence of this ash layer, particularly towards the outlet end might have contributed to differences between expected PM distribution trend and measured distribution. 


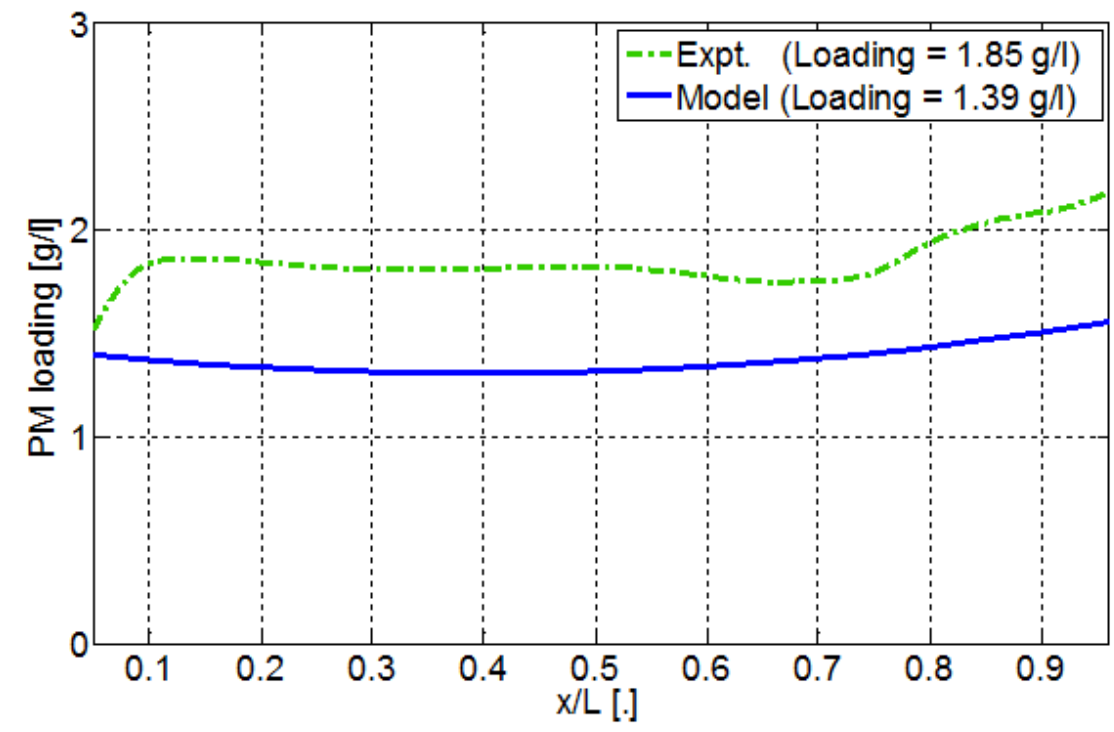

Figure 5.34: Axial PM loading distribution from experimental data [39] at the end of passive oxidation compared to model results at a similar point in the simulation of PO-B20-12

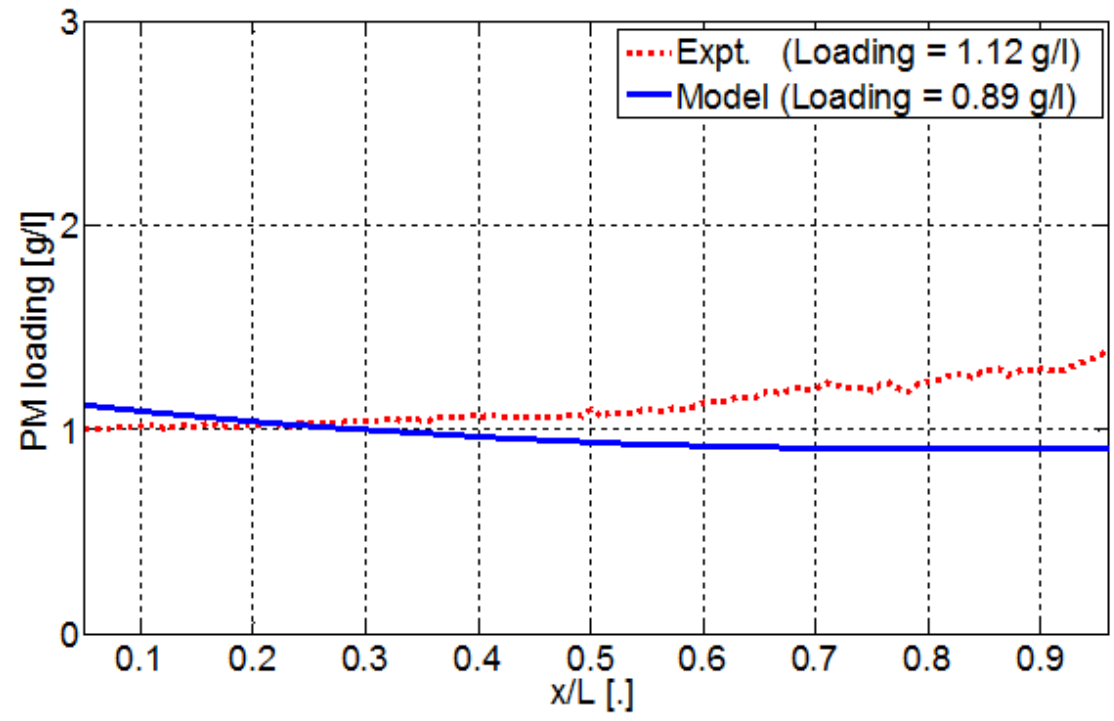

Figure 5.35: Axial PM loading distribution from experimental data [39] at the end of active regeneration compared to model results at a similar point in the simulation of AR-ULSD-1 


\subsection{Effect of Back-Diffusion of $\mathrm{NO}_{2}$ on PM Oxidation Rate}

Back-diffusion is the phenomenon where the $\mathrm{NO}_{2}$ produced in the catalyst washcoat diffuses back into the PM cake layer against the direction of flow of exhaust gas and participates in the oxidation of the PM. The effect of back-diffusion of $\mathrm{NO}_{2}$ results in an increased rate of $\mathrm{NO}_{2}$-assisted PM oxidation. Simulating the model with and without the back-diffusion of $\mathrm{NO}_{2}$ shows the effect of $\mathrm{NO}_{2}$ back-diffusion on oxidation of PM resulting from the model calibration. A modeling study was carried out on the passive oxidation experiments to quantify the effects of the diffusion of $\mathrm{NO}_{2}$ on total PM oxidized during the passive oxidation (PO) phase of all passive oxidation experiments and is shown in Table 5.4. The percentage PM oxidized decreased by 0.7-3.7 \% without diffusion considered, suggesting that the effect of back-diffusion of $\mathrm{NO}_{2}$ on PM oxidized in the PM cake layer for the test cases considered is in the 1 to $4 \%$ range. This is in contrast with findings from [59] which studied a SiC filter which concluded that at lower engine-out temperatures, the effect of back-diffusion of $\mathrm{NO}_{2}$ could be as high as $30 \%$ on the oxidation rate of PM. The reason for this could be because of the following assumptions made in the CPF model:

- Tortuosity of PM cake layer plays an important role in determining the effective diffusivities of gaseous species in the PM cake layer. The calibration of this variable was carried out as part of the procedure developed to simulate outlet $\mathrm{NO}_{2}$ concentrations and PM mass retained at the end of PO and AR stages of the eighteen PO and AR experiments by a single set of PM oxidation and nitrogen oxides oxidation kinetic parameters. Ideally, this variable has to be determined based on the PM cake layer properties which then would be excluded from the calibration.

- Effective diffusivities of the gaseous species are calculated by Equation D.5 where the Knudsen diffusivities are calculated by the Equation D.8 and molecular diffusivities are calculated by Maxwell-Stefan equations D.7 for multicomponent diffusion. The diffusion of any gaseous species takes place in a mixture of all gaseous species, both reactants and products. The use of this model to calculate molecular diffusivities could lead to discrepancies in the calculated values of effective diffusivities. Further work is needed to determine the accurate modeling of effective diffusivities of gaseous species, specifically $N O 2$, in order to verify the accuracy of the back-diffusion model.

The next chapter summarizes the findings discussed in this chapter and presents the conclusions drawn from the results obtained in this research work. 
Table 5.4: Effect of back-diffusion of $\mathrm{NO}_{2}$ on PM oxidation for all passive oxidation experiments

\begin{tabular}{|l|r|r|r|r|}
\hline \multirow{2}{*}{ Expt. name } & $\begin{array}{r}\text { PO } \\
\text { duration }\end{array}$ & $\begin{array}{r}\text { PM oxidized } \\
\text { with diffusion }\end{array}$ & $\begin{array}{r}\text { PM oxidized } \\
\text { without diffusion }\end{array}$ & Diff. \\
\cline { 2 - 5 } & {$[$ min. $]$} & {$[g]$} & {$[g]$} & {$[\%]$} \\
\hline PO-B10-14 & 100 & 4.8 & 4.6 & 3.7 \\
\hline PO-B10-15 & 80 & 21.3 & 21.1 & 0.7 \\
\hline PO-B10-16 & 43 & 20.7 & 20.4 & 1.5 \\
\hline PO-B10-17 & 80 & 20.5 & 20.3 & 0.9 \\
\hline PO-B20-12 & 80 & 26.6 & 26.4 & 0.8 \\
\hline PO-B20-13 & 42 & 25.6 & 25.4 & 1.0 \\
\hline
\end{tabular}




\section{Summary and CONCLUSIONS ${ }^{1}$}

This chapter presents a summary of the CPF model development described in Chapter 3 and the results presented in Chapter 5 from the calibration of the model to the experimental data using the calibration procedure which were both discussed in Chapter 4.

\subsection{Summary of CPF Model Development}

The 1-D CPF model [65] was described in detail in Chapter 3. The CPF model makes use of the governing equations of conservation of mass, momentum, energy and mass of individual chemical species to simulate the performance of the CPF during the passive oxidation and active regeneration experiments. The model simulates the following processes:

- Flow of exhaust gas mixture as it passes through a single inlet channel, substrate wall and outlet channel,

- Filtration of PM occurring in the substrate wall and PM cake layer,

- Oxidation of PM via $\mathrm{NO}_{2}$-assisted and thermal $\left(\mathrm{O}_{2}\right)$ means in the cake and the wall,

- Oxidation of hydrocarbons (represented in the model by $\mathrm{C}_{12} \mathrm{H}_{24}$ ), carbon monoxide $(C O)$ and nitric oxide $(N O)$,

- Back-diffusion of gaseous species $\left(\mathrm{NO}_{2}\right)$, and

- The pressure drop resulting from the inlet channel, outlet channel, cake and wall pressure drops.

\footnotetext{
${ }^{1}$ Parts of the material contained in this chapter have been published, or are currently under consideration for publication by SAE International.
} 
A procedure for the calibration of the $\mathrm{CPF}$ model was developed. Experimental data from six passive oxidation experiments [61] and twelve active regeneration experiments [4] containing experiments conducted with ULSD, B10 and B20 fuel types. These data were used to calibrate the model following the calibration procedure developed. As a result of model calibration, a single set of filtration parameters, catalytic oxidation reaction kinetics (specifically the kinetic parameters associated with the reversible reaction of $N O$ oxidation/ $\mathrm{NO}_{2}$ dissociation) and PM oxidation reaction kinetic parameters. The identified parameters were for the $\mathrm{NO}_{2}$-assisted PM oxidation in the PM cake layer and the substrate wall and the thermal $\left(\mathrm{O}_{2}\right)$ ) PM oxidation in the PM cake layer and the substrate wall [75]. Inhibition factors associated with $\mathrm{HC}$ oxidation, $\mathrm{CO}$ oxidation and $\mathrm{NO}$ oxidation were also identified for the eighteen experiments.

\subsection{Summary of CPF Model Calibration}

Calibration of the CPF model was carried out by comparing the model results with experimental data. The input data to simulate the data were:

- Exhaust gas mass flow rates,

- Exhaust gas temperature at CPF inlet,

- $\mathrm{HC}, \mathrm{NO}_{2}, \mathrm{CO}_{2}, \mathrm{O}_{2}, \mathrm{CO}, \mathrm{NO}$ and PM concentrations at $\mathrm{CPF}$ inlet, and

- Test cell conditions (temperature, barometric pressure and relative humidity).

The model results used for quantifying the correlation between the model and the experimental data were:

- Pressure drop across the CPF,

- PM mass retained in the $\mathrm{CPF}$,

- Total PM filtration efficiency of the CPF,

- Exhaust gas temperatures at the CPF outlet, and

- $\mathrm{NO}_{2}$ concentrations at the CPF outlet.

The differences between the model results and the experimental data were quantified and presented in Appendix J. The following observations were made about the agreement between the model and the experimental data: 
Using filtration parameters as shown in Table I.1, catalytic reaction kinetics as shown in Table 5.2 and PM kinetics as shown in Table 5.3:

1. Pressure drops across the CPF were calibrated to within $(-0.29 /+0.37 \mathrm{kPa})$ of the experimental data,

2. $\mathrm{PM}$ retained in the $\mathrm{CPF}$ were calibrated to within $(-2.3 /+2.6 \mathrm{~g})$ of the experimental data,

3. Total filtration efficiency of the CPF during loading stage- 2 were calibrated to within $(-1.0 /+0.9 \%)$ of the experimental data (excluding two cases (AR-ULSD-5 and AR-B20-6) where the experimental filtration efficiency reported $(95.4 \%$ and $93.5 \%$ respectively) [55] were assumed to be in error),

4. Exhaust gas temperatures out of the $\mathrm{CPF}$ during the eighteen experiments were calibrated to within $\left(-8 /+7^{\circ} \mathrm{C}\right)$ of the experimental data, and

5. $\mathrm{NO}_{2}$ concentrations out of the CPF during the eighteen experiments were calibrated to within $(-15 /+8 \mathrm{ppm})$ of the experimental data.

Using a single set of filtration parameters as shown in Table 5.1, catalytic reaction kinetics as shown in Table 5.2 and PM kinetics as shown in Table 5.3:

1. Pressure drops across the CPF were calibrated to within $(-0.26 /+0.35 \mathrm{kPa})$ of the experimental data,

2. $\mathrm{PM}$ retained in the $\mathrm{CPF}$ were calibrated to within $(-1.9 /+2.8 \mathrm{~g})$ of the experimental data,

3. Total filtration efficiency of the CPF during loading stage- 2 were calibrated to within $(-1.0 /+1.1 \%)$ of the experimental data (excluding two cases (AR-ULSD-5 and AR-B20-6) where the experimental filtration efficiency reported $(95.4 \%$ and $93.5 \%$ respectively) [55] were assumed to be in error),

4. Exhaust gas temperatures out of the $\mathrm{CPF}$ during the eighteen experiments were calibrated to within $\left(-8 /+7^{\circ} \mathrm{C}\right)$ of the experimental data, and

5. $\mathrm{NO}_{2}$ concentrations out of the $\mathrm{CPF}$ during the eighteen experiments were calibrated to within $(-15 /+8 \mathrm{ppm})$ of the experimental data. 


\subsection{Summary of Major Findings}

From the calibration of the model to the experiments conducted with three different fuel types, the major findings are:

1. Considering calibrated model data from all eighteen experiments, the fraction of PM oxidized during passive oxidation or active regeneration phase of each experiment due to $\mathrm{NO}_{2}$-assisted means to total PM oxidized showed a sigmoid fit (Figure 5.21) with increasing fraction of total PM oxidation being via thermal $\left(\mathrm{O}_{2}\right)$ means.

2. From an analysis of total PM reaction rates for the three different fuel types studied, the $\mathrm{NO}_{2}$-assisted PM oxidation increased with increasing temperature and $\mathrm{NO}_{2}$ concentrations. The temperature at which thermal oxidation equals $\mathrm{NO}_{2}$-assisted is lower for higher biodiesel content (shown in Figures 5.22, 5.23 and 5.24). This is due to lower activation energy for the $\mathrm{NO}_{2}$-assisted reaction for higher biodiesel level (as shown in Table 5.3).

3. From an analysis of total reaction rates for the three different fuel types studied, a total reaction rate equal to an active regeneration experiment can be obtained by a lower CPF inlet temperature and higher CPF inlet $\mathrm{NO}_{2}$ concentration (as shown in Figures 5.28, 5.29 and 5.30).

4. From a study conducted on the effect of PM oxidation on PM cake layer thickness using calibrated model results, both the passive oxidation and active regeneration show non-uniform PM loading in the cake at the end of loading, with the middle being loaded less than the inlet end or the outlet end. After the passive oxidation, this profile was maintained (as shown in Figure 5.31) due to low total PM oxidation rate in this case. However, after active regeneration, the profile showed the outlet end being the area of minimum PM cake thickness (as shown in Figure 5.32) due to higher PM oxidation rates at the outlet end during active regeneration in turn due to higher PM mass available and higher substrate wall temperatures. Both trends qualitatively agree with experimental data obtained using PM imaging technique conducted at MTU [39] as shown in Figures 5.34 and 5.35.

5. Back-diffusion of $\mathrm{NO}_{2}$ contributed to a 1 to $4 \%$ difference of the total PM oxidized during the passive oxidation experiments (as shown in Table 5.4).

\subsection{Conclusions}

The specific conclusions from this research work are: 
1. Engine experimental data from passive oxidation [54] and active regeneration [55] experiments were used to determine the filtration and kinetic parameters for the $\mathrm{CPF}$ model developed.

2. The PM oxidation kinetic parameters obtained from calibration of the model to the experimental data for three different fuel-types show that the total reaction rates of PM oxidation increase with increasing biodiesel blend level (ULSD < B10 < B20) and are shown in Table 5.3 and in Figures 5.25, 5.26 and 5.27.

3. An analysis of the fraction of PM oxidized by thermal $\left(\mathrm{O}_{2}\right)$ to total $\left(\mathrm{NO}_{2}\right.$-assisted + thermal) PM oxidation was carried out. For the aftertreatment system studied in this research work, $80 \%$ of the total PM reaction rate is via $\mathrm{NO}_{2}$-assisted oxidation at $430^{\circ} \mathrm{C}$. At $486^{\circ} \mathrm{C}$, the oxidation is $50 \% \mathrm{NO}_{2}$-assisted and $50 \%$ thermal, and $80 \%$ is thermal at $516^{\circ} \mathrm{C}$.

4. The gaseous species kinetics for $N O, C O$ and $H C$ oxidation including the inhibition parameters have been determined for the CPF studied in this research work and are shown in Table 5.2.

5. The PM oxidation models have been used to parametrically define the characteristic trends of the $\mathrm{NO}_{2}$-assisted and thermal PM oxidation for a range of temperatures and $\mathrm{NO}_{2}$ concentrations to illustrate how $\mathrm{NO}_{2}$ concentrations and temperature can be used to increase the passive PM oxidation in future applications for ULSD, B10 and B20 fuels. 



\section{REFERENCES}

(1) Dieselnet - online resource. http: / / www. dieselnet. com/tech/dpf_top. html. Accessed: 2005.

(2) Dieselnet - online resource. http://www. dieselnet.com/tech/cat_scr. php. Accessed: 2013.

(3) Dieselnet - online resource. http://www.dieselnet.com/tech/cat_doc. html. Accessed: 2011.

(4) J. M. Pidgeon, J. H. Johnson, and J. D. Naber. An Experimental Investigation into Particulate Matter Oxidation in a Catalyzed Particulate Filter with Biodiesel Blends on an Engine during Active Regeneration. SAE Technical Paper 2013-01-0521, 2013.

(5) S. H. Oh, J. S. Macdonald, G. L. Vaneman, and L. L. Hegadus. Mathematical Modeling of Fibrous Filters for Diesel Particulates - Theory and Experiment. SAE Technical Paper 810113, 1981.

(6) H. Oh and J. C. Cavendish. Transients of Monolithic Catalytic Converters: Response to Step Changes in Feedstream Temperature as Related to Controlling Automobile Emissions. Ind. Eng. Chem. Prod. Res. Dev., 21:29-37, 1982.

(7) F. Shadman and E. Bissett. Analysis of Thermal Regeneration of Fibrous Diesel-Particulate Filters. Ind. Eng. Chem. Proc. Res. Dev, 22(2):203-208, 1983.

(8) E. Bissett. Mathematical Model of the Thermal Regeneration of a Wall-Flow Monolith Diesel Particulate Filter. Chemical Engineering Science, 39, 1984.

(9) F. Shadman. Kinetics of Soot Combustion During Regeneration of Surface Filters. Combustion Science and Technology, 36, 1989.

(10) E. Pauli, G. Lepperhoff, and F. Pischinger. The Description of the Regeneration Behavior of Diesel Particulate Traps with the Aid of a Mathematical Model. SAE Technical Paper 830180, 1983. 
(11) A. G. Konstandopoulos and J.H. Johnson. Wall-Flow Diesel Particulate Filters - Their Pressure Drop and Collection Efficiency. SAE Technical Paper 890405, 1989.

(12) K. W. Lee and J. A. Gieseke. Collection of Aerosol Particles by Packed Beds. Environmental Science and Technology, 13(4), 1979.

(13) A. G. Konstandopoulos, M. Kostoglou, E. Skaperdas, E. Papaioannou, D. Zarvalis, and E. Kladopoulou. Fundamental Studies of Diesel Particulate Filters : Transient Loading, Regeneration and Aging. SAE Technical Paper 2000-01-1016, 2000.

(14) M. Masoudi, A. G. Konstandopoulos, M. S. Nikitidis, E. Skaperdas, D. Zarvalis, E. Kladopoulou, and C. Altiparmakis. Validation of a Model and Development of a Simulator for Predicting the Pressure Drop of Diesel Particulate Filters. SAE Technical Paper 2001-01-0911, 2001.

(15) A.G. Konstandopoulos, E. Skaperdas, and M. Masoudi. Inertial Contributions to the Pressure Drop of Diesel Particulate Filters. SAE Technical Paper 2001-01-0909, 2001.

(16) A. G. Konstandopoulos, M. Kostoglou, and P. Housiada. Spatial Non-Uniformities in Diesel Particulate Trap Regeneration. SAE Technical Paper 2001-01-0908, 2001.

(17) Y. Miyairi, S. Miwa, F. Abe, Z. Xu, and Y. Nakasuji. Numerical Study on Forced Regeneration of Wall-Flow Diesel Particulate Filters. SAE Technical Paper 2001-01-0912, 2001.

(18) A. G. Konstandopoulos, M. Kostoglou, P. Housiada, N. Vlachos, and D. Zarvalis. Multichannel Simulation of Soot Oxidation in Diesel Particulate Filters. SAE Paper No. 2003-01-0839, 2003.

(19) C. N. Opris and J. H. Johnson. A 2-D Computational Model Describing the Flow and Filtration Characteristics of a Ceramic Diesel Particulate Trap. SAE Technical Paper 980545, 1998.

(20) C. N. Opris and J. H. Johnson. A 2-D Computational Model Describing the Heat Transfer, Reaction Kinetics and Regeneration Characteristics of a Ceramic Diesel Particulate Trap. SAE Technical Paper 980546, 1998.

(21) C. Depcik and D. Assanis. Simulating Area Conservation and the Gas-Wall Interface for One-Dimensional Based Diesel Particulate Filter Models. ASME Journal of Engineering for Gas Turbines and Power, 2008.

(22) G. C. Koltsakis, O. A. Haralampous, C. K. Dardiotis, Z. C. Samaras, C. D. Vogt, E. Ohara, Y. Watanabe, and T. Mizutani. Performance of Catalyzed Particulate Filters Without Upstream Oxidation Catalyst. SAE Technical Paper 2005-01-0952, 2005. 
(23) G. C. Koltsakis, O. A. Haralampous, N. K. Margaritis, Z. C. Samaras, C. D. Vogt, E. Ohara, Y. Watanabe, and T. Mizutani. 3-Dimensional Modelling of the Regeneration in SiC Particulate Filters. SAE Technical Paper 2005-01-0953, 2005.

(24) Y. Yi. Simulating the Soot Loading in Wall-flow DPF Using a Three-Dimensional Macroscopic Model. SAE Technical Paper 2006-01-0264, 2006.

(25) C. Hinterberger, M. Olesen, and R. Kaiser. 3D Simulation of Soot Loading and Regeneration of Diesel Particulate Filter Systems. SAE Technical Paper 2007-01-1143, 2007.

(26) M. Frey, G. Wenninger, B. Krutzsch, G. C. Koltsakis, O. A. Haralampous, and Z. C. Samaras. 2D Simulation of the Regeneration Performance of a Catalysed DPF for Heavy-Duty Applications. Topics in Catalysis, 42-43, 2007.

(27) Exothermia - online resource. http://exothermia.com/index.php? option=com_content\&task=view\&id=17\&Itemid=31. Accessed: 2012.

(28) G. C. Koltsakis, C. K. Dardiotis, Z. C. Samaras, M. Frey, G. Wenninger, B. Krutzsch, and O. A. Haralampous. Model-Based Optimization of Catalyst Zoning in Diesel Particulate Filters. SAE Technical Paper 2008-01-0445, 2008.

(29) G. C. Koltsakis and O. A. Haralampous. Multi-Dimensional Modeling of Catalyzed Particulate Filters. Technical report, 6th International CTI Forum Exhaust Systems at Nurtingen. (Germany), 2008.

(30) M. C. Law, A. Clarke, C. P. Garner, and A. M. Williams. A Finite-Volume-Based Two-Dimensional Wall-Flow Diesel Particulate Filter Regeneration Model. Proc. IMechE Part D: J. Auto. Engg., 222, 2008.

(31) M. Schejbal, M. Marek, M. Kubicek, and P. Koci. Modelling of Diesel Filters for Particulates Removal. Chemical Enginering Journal, 154:219-230, 2009.

(32) P. Tandon, A. K. Heibel, J. Whitmore, N. Kekre, and K. Chithapragada. Measurement and Prediction of Filtration Efficiency Evolution of Soot Loaded Diesel Particulate Filters. Reviews in Chemical Engineering, 65(16):4751-4760, 2010.

(33) D. Zhong, S. He, P. Tandon, M. Moreno, and T. Boger. Measurement and Prediction of Filtration Efficiency Evolution of Soot Loaded Diesel Particulate Filters. SAE Technical Paper 2012-01-0363, 2012.

(34) G. Koltsakis, O. Haralampous, C. Depcik, and J. Ragone. Catalyzed Diesel Particulate Filter Modeling. Reviews in Chemical Engineering, 29(1):1-61, 2013. 
(35) Soot Loading and Regeneration of Diesel Particulate Filters - CD-adapco - online resource. http://www.cd-adapco.com/press_room/dynamics/26/ auto2.html. Accessed: 2008.

(36) E. Ohara, Y. Mizuno, Y. Miyairi, T. Mizutani, K. Yuuki, Y. Noguchi, T. Hiramatsu, M. Makino, A. Takahashi, H. Sakai, M. Tanaka, A. Martin, S. Fujii, P. Busch, T. Toyoshima, T. Ito, I. Lappas, and C. D. Vogt. Filtration Behavior of Diesel Particulate Filters (1). SAE Technical Paper 2007-01-0921, 2007.

(37) Z. G. Liu and R. K. Miller. Flow Distributions and Pressure Drops of Wall-Flow Diesel Particulate Filters. SAE Technical Paper 2002-01-1311, 2002.

(38) F. Piscaglia, C. J. Rutland, and D. E. Foster. Development of a CFD Model to Study the Hydrodynamic Characteristics and the Soot Deposition Mechanism on the Porous Wall of a Diesel Particulate Filter. SAE Technical Paper 2005-01-0963, 2005.

(39) R. K. Foley. Experimental Investigation into Particulate Matter Distribution in Catalyzed Particulate Filters Using a 3D Terahertz Wave Scanner. Master's thesis, Michigan Technological University, 2013.

(40) J. Nagle and R. F. Strickland-Constable. Oxidation of Carbon between 1000-2000 C. Fifth Carbon Conference, 1961.

(41) M. J. Murphy, L. J. Hillenbrand, D. A. Trayser, and J. H. Wasser. Assessment of Diesel Particulate Control - Direct and Catalytic Oxidation. SAE Technical Paper 810112, 1981.

(42) Z. Du, A. F. Sarofim, and J. P. Longwell. Kinetic Measurement and Modeling of Carbon Oxidation. Energy and Fuels, 5, 1991.

(43) J. P. A. Neeft, O. P. vanPruissen, M. Makee, and J. A. Mouljin. Catalysts for the Oxidation of Soot from Diesel Exhaust Gases II - Contact between Soot and Catalyst under Practical Conditions. Applied Catalysis B : Environmental, 12, 1997.

(44) B. R. Stanmore, J. F. Brilhac, and P. Gilot. The Oxidation of Soot: a Review of Experiments, Mechanisms and Models. Carbon, 39, 2001.

(45) F. Jacquot, V. Logie, J. F. Brilhac, and P. Gilot. Kinetics of the Oxidation of Carbon Black by $\mathrm{NO}_{2}$ : Influence of the presence of water and oxygen. Carbon, 40, 2002.

(46) A. G. Konstandopoulos and M. Kostoglou. Periodically Reversed Flow Regeneration of Diesel Particulate Traps. SAE Technical Paper 1999-01-0469, 1999.

(47) E. Papaioannou, D. Zarvalis, and A. G. Konstandopoulos. Influence of NO2 on soot particles oxidation. Proc. Second Panhellenic Symposium of Chemical Engineers, page $325,1999$. 
(48) A. Yezerets, N. W. Currier, D. H. Kim, H. A. Eadler, W. S. Epling, and C. H. F. Peden. Differential Kinetic Analysis of Diesel Particulate Matter (Soot) Oxidation by Oxygen using a Step-response Technique. Applied Catalysis B: Environmental, 50:120-129, 2005.

(49) A. Strzelec, T. Toops, C. S. Daw, D. Foster, and C. Rutland. Diesel Particulate Oxidation Model: Combined Effects of Volatiles and Fixed Carbon Combustion. SAE Technical Paper 2010-01-2127, 2010.

(50) I. P. Kandylas, O. A. Haralampous, and G. C. Koltsakis. Diesel Soot Oxidation with NO2: Engine Experiments and Simulations. Industrial Engineering Chemistry Research, 41:5372-5384, 2002.

(51) I. P. Kandylas and G. C. Koltsakis. NO2-assisted Regeneration of Diesel Particulate Filters : A Modeling Study. Industrial Engineering Chemistry Research, 41:2115-2123, 2002.

(52) O. A. Haralampous, G. C. Koltsakis, and Z. C. Samaras. Partial Regenerations in Diesel Particulate Filters. SAE Technical Paper 2003-01-1881, 2003.

(53) O. A. Haralampous, G. C. Koltsakis, Z. C. Samaras, C. D. Vogt, E. Ohara, Y. Watanabe, and T. Mizutani. Reaction and Diffusion Phenomena in Catalyzed Diesel Particulate Filters. SAE Technical Paper 2004-01-0696, 2004.

(54) K. L. Shiel. A Study of the Effect of Biodiesel Fuel on Passive Oxidation in a Catalyzed Particulate Filter. Master's thesis, Michigan Technological University, 2012.

(55) J. M. Pidgeon. An Experimental Investigation into the Effects of Biodiesel Blends on Particulate Matter Oxidation in a Catalyzed Particulate Filter during Active Regeneration. Master's thesis, Michigan Technological University, 2012.

(56) H. Mohammed, A. P. Triana, S. L. Yang, and J. H. Johnson. An Advanced 1D 2-layer Catalyzed Diesel Particulate Filter Model to Simulate : Filtration by the Wall and Particulate Cake, Oxidation in the Wall and Particulate Cake by $\mathrm{NO}_{2}$ and $\mathrm{O}_{2}$, and Regeneration by Heat Addition. SAE Technical Paper 2006-01-0467, 2006.

(57) S. E. Voltz, C. R. Morgan, D. Liederman, and S. M. Jacob. Kinetic Study of Carbon Monoxide and Propylene Oxidation on Platinum Catalysts. Ind. Eng. Chem. Prod. Res. Dev., 12(4), 1973.

(58) L. Olsson, H. Persson, E. Fridell, M. Skoglundh, and B. Andersson. A Kinetic Study of $\mathrm{NO}$ Oxidation and $\mathrm{NO}_{x}$ Storage on $\mathrm{Pt} / \mathrm{Al}_{2} \mathrm{O}_{3}$ and $\mathrm{Pt} / \mathrm{BaO} / \mathrm{Al}_{2} \mathrm{O}_{3}$. Journal of Physical Chemistry B, 105, 2001. 
(59) O. A. Haralampous and G. C. Koltsakis. Back-Diffusion Modeling of NO2 in Catalyzed Diesel Particulate Filters. Ind. Eng. Chem. Res., 2004.

(60) C. R. Hutton, J. H. Johnson, J. D. Naber, and J. M. Keith. Procedure Development and Experimental Study of Passive Particulate Matter Oxidation in a Diesel Catalyzed Particulate Filter. SAE Technical Paper 2012-01-0851, 2012.

(61) K. L. Shiel, J. D. Naber, J. H. Johnson, and C. R. Hutton. Catalyzed Particulate Filter Passive Oxidation Study with ULSD and Biodiesel Blended Fuel. SAE Technical Paper 2012-01-0837, 2012.

(62) C. T. Huynh, J. H. Johnson, S. L. Yang, S. T. Bagley, and J. R. Warner. A One-Dimensional Computational Model for Studying the Filtration and Regeneration Characteristics of a Catalyzed Wall-Flow Diesel Particulate Filter. SAE Technical Paper 2003-01-0841, 2003.

(63) C. T. Huynh. A Study of the Filtration and Regeneration Characteristics of a Catalyzed Wall Flow Diesel Particulate Filter: One Dimensional Model Calibrated and Validated with Experimental Data. Master's thesis, Michigan Technological University, 2002.

(64) H. Mohammed. The Filtration and Oxidation Characteristics of a Diesel Oxidation Catalyst and a Catalyzed Particulate Filter: Development of a 1-D 2-Layer Model. Master's thesis, Michigan Technological University, 2005.

(65) K. C. Premchand, J. H. Johnson, and S. L. Yang. Development of a 1-D Catalyzed Diesel Particulate Filter Model for Simulation of the Oxidation of Particulate Matter and Gaseous Species During Passive Oxidation and Active Regeneration. SAE Technical Paper 2013-01-1574, 2013.

(66) R. B. Bird, W. E. Stewart, and E. N. Lightfoot. Transport Phenomena. John Wiley and Sons, 1960.

(67) K. A. Hoffmann and S. T. Chiang. Computational Fluid Dynamics - Volume 1 - Fifth Edition. EES, 2000.

(68) S. V. Patankar. Numerical Heat Transfer and Fluid Flow. Taylor \& Francis, 1980.

(69) W. H. Press, S. A. Teukolsky, W. T. Vetterling, and B. P. Flannery. Numerical Recipes: The Art of Scientific Computing (Third Edition). Cambridge University Press, 2007.

(70) C. Orr. Filtration : Principles and Practices - Part 1. Chemical Processing and Engineering, Marcel Drekker Incorporated, NY, 1981. 
(71) H. Darcy. Les Fontaines Publiques de la Ville de Dijon (The Public Fountains of the City of Dijon), Dalmont, Paris. SAE technical paper series. Society of Automotive Engineers, 1856.

(72) A. G. Konstandopoulos, E. Skaperdas, and M. Masoudi. Microstructural Properties of Soot Deposits in Diesel Particulate Traps. SAE Technical Paper 2002-01-1015, 2002.

(73) X. S. Song, H. S. Surenahalli, J. D. Naber, G. G. Parker, and J. H. Johnson. Experimental and Modeling Study of a Diesel Oxidation Catalyst (DOC) under Transient and CPF Active Regeneration Conditions. SAE Technical Paper 2013-01-1046, 2013.

(74) R. K. Foley, D. Naber, J., and J. H. Johnson. Development of the Methodology for Using a Terahertz Wave Scanner to Determine the 3D PM Distribution in a Catalyzed Particulate Filter. Manuscript submitted for publication to SAE International., 2014.

(75) K. C. Premchand, H. S. Surenahalli, and J. H. Johnson. Particulate Matter and Nitrogen Oxides Kinetics Based on Engine Experimental Data for a Catalyzed Diesel Particulate Filter. Manuscript submitted for publication to SAE International., 2014.

(76) M. J. Moran and H. N. Shapiro. Fundamentals of Engineering Thermodynamics $\left(5^{\text {th }}\right.$ edition. John Wiley and Sons, Inc., 2004.

(77) R. Aris. The Mathematical Theory of Diffusion and Reaction in Permeable Catalysts. Oxford University Press, 1975. 


\section{APPENDIX A. DERIVATION OF Conservation of Mass of Exhaust Gas MIXTURE}

Figure A.1 shows a control volume in the inlet channel of the CPF. Axial convection into the control volume through the left face, out of the control volume through the right face and out of the control volume through the 4 wall faces is considered.

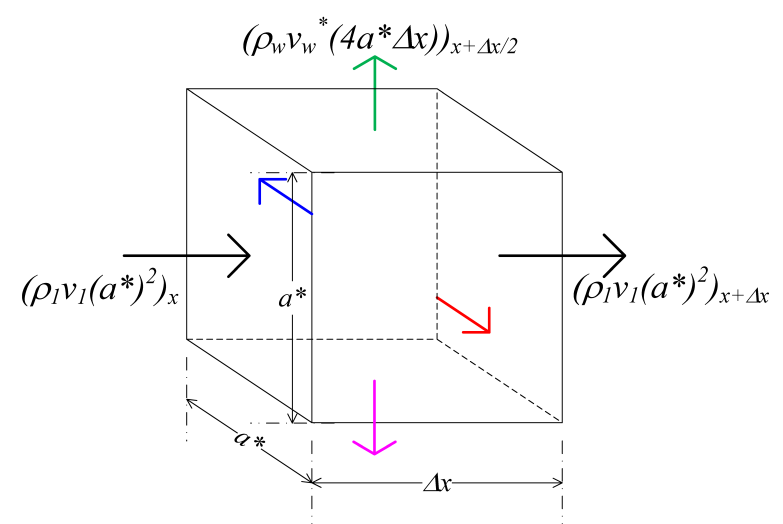

Figure A.1: Diagram showing dimensions of inlet channel control volume and components of mass balance in inlet channel control volume

The general mathematical expression for conservation of mass is:

$$
\frac{d\left(m_{1}\right)}{d t}=\left.\rho_{1} v_{1}\left(a^{*}\right)^{2}\right|_{x}-\left.\rho_{1} v_{1}\left(a^{*}\right)^{2}\right|_{x+\Delta x}-\left.\left(4 a^{*} \Delta x\right) \rho_{w} v_{w}^{*}\right|_{x+\frac{\Delta x}{2}}
$$

where $m_{1}$ is the mass of the exhaust gas mixture contained in the inlet channel control volume of dimensions $\left(a^{*} \times a^{*} \times \Delta x\right)$ as shown in Figure A.1, $\rho_{1}$ is the density of the exhaust gas mixture in the control volume, $v_{1}$ is the velocity of the exhaust gas mixture, $a^{*}$ is the effective width of the inlet channel control volume, $\Delta x$ is the discretized length 
of the inlet channel control volume, $\rho_{w}$ is the density of the exhaust gas mixture in the cake+cat+wall control volume and $v_{w}^{*}$ is the entry velocity of the exhaust gas mixture into the cake+cat+wall control volume.

At quasi-steady state, $\frac{d\left(m_{1}\right)}{d t}=0$. So, equation (A.1) becomes:

$$
\left.\rho_{1} v_{1}\left(a^{*}\right)^{2}\right|_{x}-\left.\rho_{1} v_{1}\left(a^{*}\right)^{2}\right|_{x+\Delta x}-\left.\left(4 a^{*} \Delta x\right) \rho_{w} v_{w}^{*}\right|_{x+\frac{\Delta x}{2}}=0
$$

Assuming $a^{*}$ to be constant in the control volume, equation (A.2) can be re-written as:

$$
\left(a^{*}\right)^{2} \Delta x \frac{\left.\rho_{1} v_{1}\right|_{x}-\left.\rho_{1} v_{1}\right|_{x+\Delta x}}{\Delta x}=\left.\left(\frac{4}{a^{*}}\left(a^{*}\right)^{2} \Delta x\right) \rho_{w} v_{w}^{*}\right|_{x+\frac{\Delta x}{2}}
$$

Dividing equation (A.3) throughout by $\left(a^{*}\right)^{2} \Delta x$ and re-writing in differential form,

$$
-\frac{d}{d x}\left(\rho_{1} v_{1}\right)=\frac{4}{a^{*}} \rho_{w} v_{w}^{*}
$$

In the cake+cat+wall control volume (see Figure A.2), a transient mass balance of the exhaust gas mixture considering convection in the transverse direction through the top and bottom faces (convection in the $\mathrm{x}$ direction is neglected) can be written as:

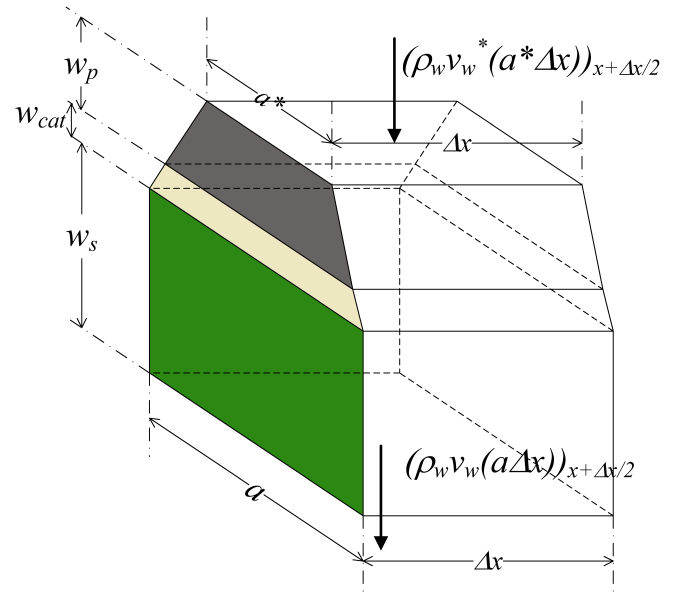

Figure A.2: Diagram showing dimensions of the cake+cat+wall control volume and components of mass balance in the cake+cat+wall control volume

$$
\frac{d\left(m_{w}\right)}{d t}=\left.\rho_{w} v_{w}^{*} a^{*} \Delta x\right|_{x+\frac{\Delta x}{2}}-\left.\rho_{w} v_{w} a \Delta x\right|_{x+\frac{\Delta x}{2}}
$$


where $m_{w}$ is the mass of the exhaust gas mixture in the cake+cat+wall control volume, $\rho_{w}$ is the density of the exhaust gas mixture in the cake+cat+wall control volume, $v_{w}^{*}$ is the entry velocity of the exhaust gas mixture into the cake+cat+wall control volume and $v_{w}$ is the exit velocity of the exhaust gas mixture out of the cake+cat+wall control volume.

Assuming quasi-steady state, or $\frac{d\left(m_{w}\right)}{d t}=0$,

$$
\left.\rho_{w} v_{w}^{*} a^{*} \Delta x\right|_{x+\frac{\Delta x}{2}}=\left.\rho_{w} v_{w} a \Delta x\right|_{x+\frac{\Delta x}{2}}
$$

or

$$
v_{w}^{*}=\frac{a}{a^{*}} v_{w}
$$

Substituting equation (A.7) into equation (A.4) and changing signs, we get:

$$
\frac{d}{d x}\left(\rho_{1} v_{1}\right)=-\frac{4}{a^{*}} \frac{a}{a^{*}} \rho_{w} v_{w}
$$

or

$$
\frac{d}{d x}\left(\rho_{1} v_{1}\right)=-\frac{4}{a}\left(\frac{a}{a^{*}}\right)^{2} \rho_{w} v_{w}
$$

In the outlet channel control volume, transient mass balance can be carried out considering axial convection through the left and right faces and transport of mass from the wall faces as shown in Figure A.3 as:

$$
\frac{d\left(m_{2}\right)}{d t}=\left.\rho_{2} v_{2} a^{2}\right|_{x}-\left.\rho_{2} v_{2} a^{2}\right|_{x+\Delta x}+\left.(4 a \Delta x) \rho_{w} v_{w}\right|_{x+\frac{\Delta x}{2}}
$$

where $m_{2}$ is the mass of the exhaust gas mixture contained in the outlet channel control volume of dimensions ( $a \mathrm{x} a \mathrm{x} \Delta x$ ), $\rho_{2}$ is the density of the exhaust gas mixture in the outlet channel control volume and $v_{2}$ is the velocity of the exhaust gas mixture in the outlet channel control volume.

At quasi-steady state, the temporal gradient term can be dropped, resulting in: 


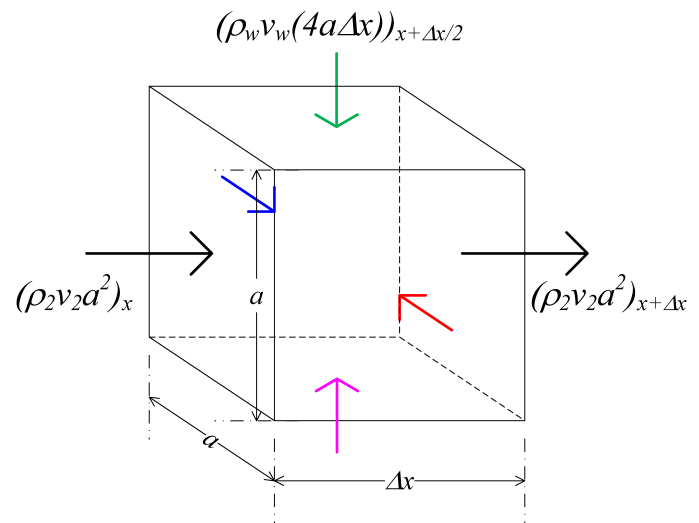

Figure A.3: Diagram showing dimensions of outlet channel control volume and components of mass balance in outlet channel control volume

$$
\left.\rho_{2} v_{2} a^{2}\right|_{x}-\left.\rho_{2} v_{2} a^{2}\right|_{x+\Delta x}+\left.(4 a \Delta x) \rho_{w} v_{w}\right|_{x+\frac{\Delta x}{2}}=0
$$

Equation (A.11) can be divided throughout by $a^{2} \Delta x$ and re-written in differential form as:

$$
\frac{\left.\rho_{2} v_{2}\right|_{x}-\left.\rho_{2} v_{2}\right|_{x+\Delta x}}{\Delta x}=-\left.\frac{4}{a} \rho_{w} v_{w}\right|_{x+\frac{\Delta x}{2}}
$$

or in differential form,

$$
-\frac{d}{d x}\left(\rho_{2} v_{2}\right)=-\frac{4}{a} \rho_{w} v_{w}
$$

Changing signs,

$$
\frac{d}{d x}\left(\rho_{2} v_{2}\right)=\frac{4}{a} \rho_{w} v_{w}
$$




\section{APPENDIX B. DERIVATION OF EQUATIONS For Calculating The Gaseous Velocity FIELD}

Velocity of the exhaust gas mixture as it passes through the CPF is an important variable that needs to be calculated in order to further calculate the filtration (or wall-flow) velocity at each axial location in the particulate filter. This appendix provides details of the derivations involved in arriving at a solution of this variable using basic governing equations.

\section{Governing Equations Used}

Conservation of mass of the exhaust gas mixture as it passes through the inlet channel is derived in this section. Each inlet channel is open at the inlet side, closed by the end plug at the outlet side and also bound by four sides where the exhaust gas mixture flows in the 'into-the-wall' or transverse direction, owing to the porous nature of the substrate wall.

Mass balance for the inlet and outlet channel in the single channel framework at the quasi-steady state can be arrived at from first principles as shown in Appendix A (please see Equations (A.9) and (A.14)) can be expressed as[8,11]:

$$
\begin{aligned}
\frac{d}{d x}\left(\rho_{1} v_{1}\right) & =-\frac{4}{a}\left(\frac{a}{a^{*}}\right)^{2} \rho_{w} v_{w} \\
\frac{d}{d x}\left(\rho_{2} v_{2}\right) & =\frac{4}{a} \rho_{w} v_{w}
\end{aligned}
$$

where $a$ is the width of the 'clean' outlet channel, $a^{*}$ is the effective width of the inlet channel, $v_{1}$ is the velocity of the exhaust gas in the inlet channel, $\rho_{1}$ is the density of the exhaust gas in the inlet channel, $v_{2}$ is the velocity of the exhaust gas in the outlet channel, $\rho_{2}$ is the density of the exhaust gas in the outlet channel, $v_{w}$ is the exit velocity of the exhaust 
gas from the cake+cat+wall control volume and $\rho_{w}$ is the density of the exhaust gas in the cake+cat+wall control volume.

Differentiating equation (B.1b) with respect to the independent variable $x$, we get:

$$
\frac{d^{2}}{d x^{2}}\left(\rho_{2} v_{2}\right)=\frac{4}{a} \frac{d}{d x}\left(\rho_{w} v_{w}\right)
$$

or:

$$
\frac{d}{d x}\left(\rho_{w} v_{w}\right)=\frac{a}{4} \frac{d^{2}}{d x^{2}}\left(\rho_{2} v_{2}\right)
$$

It is known from wall mass conservation (please see Equation (A.7)) that:

$$
v_{w}^{*}=v_{w} \frac{a}{a^{*}}
$$

By combining equations (B.1a) and (B.1b), we can write:

$$
\left(\frac{a^{*}}{a}\right)^{2} \frac{d}{d x}\left(\rho_{1} v_{1}\right)+\frac{d}{d x}\left(\rho_{2} v_{2}\right)=-\frac{4}{a} \rho_{w} v_{w}+\frac{4}{a} \rho_{w} v_{w}=0
$$

At this point, it is assumed that $\left(\frac{a^{*}}{a}\right)^{2} \approx 1$. Then, eqn (B.5) can be further simplified and re-written as:

$$
\frac{d}{d x}\left(\rho_{1} v_{1}\right)+\frac{d}{d x}\left(\rho_{2} v_{2}\right)=0
$$

or

$$
\frac{d}{d x}\left(\rho_{1} v_{1}+\rho_{2} v_{2}\right)=0
$$

Integrating,

$$
\rho_{1} v_{1}+\rho_{2} v_{2}=C
$$

where $C$ is a constant, which can be obtained from examining the boundary conditions for the given problem setup. At the inlet end, i.e., $(x=0)$, we have:

$$
\begin{aligned}
\rho_{1} v_{1} & =\frac{\dot{m}}{n_{\text {cells }}\left(a^{*}\right)^{2}} \\
\rho_{2} v_{2} & =0
\end{aligned}
$$

where $\dot{m}$ is the mass flow rate into the particulate filter and $n_{\text {cells }}$ is the number of inlet cells of the particulate filter. Hence, by substituting, we obtain:

$$
\left(\rho_{1} v_{1}+\rho_{2} v_{2}\right)=\frac{\dot{m}}{n_{\text {cells }}\left(a^{*}\right)^{2}}
$$


$\frac{\dot{m}}{n_{\text {cells }}\left(a^{*}\right)^{2}}$ is hereafter referred to as $M$. So Equation (B.8) can be re-written as:

$$
\begin{gathered}
\rho_{1} v_{1}=M-\rho_{2} v_{2} \\
v_{1}=\frac{M-\rho_{2} v_{2}}{\rho_{1}}
\end{gathered}
$$

Axial momentum conservation equations for the exhaust gas in the inlet channel and outlet channel can be expressed as:

$$
\begin{aligned}
& \frac{d}{d x}\left(\rho_{1} v_{1}^{2}\right)=-\frac{d P_{1}}{d x}-F \frac{\mu_{1} v_{1}}{\left(a^{*}\right)^{2}} \\
& \frac{d}{d x}\left(\rho_{2} v_{2}^{2}\right)=-\frac{d P_{2}}{d x}-F \frac{\mu_{2} v_{2}}{a^{2}}
\end{aligned}
$$

where $P$ is the absolute pressure of the exhaust gas, and $\mu$ is the dynamic viscosity of the exhaust gas mixture. The right-hand side terms in Equations (B.13a) and (B.13b) represent the pressure loss and viscous drag loss along the length of the channel ( $x$ direction). The numerical solution of these equations provides a way to estimate the friction losses in the inlet and outlet channels. It is also known that according to D'Arcy's law,

$$
P_{1}-P_{2}=\mu_{w} v_{w}\left(\frac{w_{p}}{k_{p}}+\frac{w_{s}}{k_{s}}\right)
$$

where $P_{1}$ is the absolute pressure in the inlet channel, $P_{2}$ is the absolute pressure in the outlet channel, $\mu_{w}$ is the dynamic viscosity of the exhaust gas mixture inthe cake+cat+wall control volume, $v_{w}$ is the exit velocity of the exhaust gas mixture from the cake+cat+wall control volume, $w_{p}$ is the thickness of the PM cake layer, $k_{p}$ is the instantaneous permeability of the PM cake layer, $w_{s}$ is the thickness of the substrate wall and $k_{s}$ is the equivalent permeability of the substrate wall.

\section{Combining the Governing Equations}

Equation (B.13a) - equation (B.13b) $\rightarrow$ :

$$
\frac{d}{d x}\left(\rho_{1} v_{1}^{2}\right)-\frac{d}{d x}\left(\rho_{2} v_{2}^{2}\right)=-\left(\frac{d P_{1}}{d x}-\frac{d P_{2}}{d x}\right)-\left(F \frac{\mu_{1} v_{1}}{\left(a^{*}\right)^{2}}-F \frac{\mu_{2} v_{2}}{a^{2}}\right)
$$

or

$$
\frac{d}{d x}\left(\rho_{1} v_{1}^{2}-\rho_{2} v_{2}^{2}\right)=-\frac{d}{d x}\left(P_{1}-P_{2}\right)-F\left(\frac{\mu_{1} v_{1}}{\left(a^{*}\right)^{2}}-\frac{\mu_{2} v_{2}}{a^{2}}\right)
$$


The goal from here on is to re-write all terms in equation (B.16) as functions of $\left(\rho_{2} v_{2}\right)$. Equation (B.16) can re-written as:

$$
\frac{d\left(\frac{\left(\rho_{1} v_{1}\right)^{2}}{\rho_{1}}-\frac{\left(\rho_{2} v_{2}\right)^{2}}{\rho_{2}}\right)}{d x}=-\frac{d\left(P_{1}-P_{2}\right)}{d x}-F\left(\frac{\mu_{1} v_{1}}{\left(a^{*}\right)^{2}}-\frac{\mu_{2} v_{2}}{a^{2}}\right)
$$

Using equations (B.11), (B.12) and D’Arcy's law (B.14), we can re-write equation (B.17) as:

$$
\frac{d\left(\frac{\left(M-\rho_{2} v_{2}\right)^{2}}{\rho_{1}}-\frac{\left(\rho_{2} v_{2}\right)^{2}}{\rho_{2}}\right)}{d x}=-\frac{d\left(\mu_{w} v_{w}\left(\frac{w_{p}}{k_{p}}+\frac{w_{s}}{k_{s}}\right)\right)}{d x}-F\left(\frac{\mu_{1} \frac{M-\rho_{2} v_{2}}{\rho_{1}}}{\left(a^{*}\right)^{2}}-\frac{\mu_{2} \frac{\rho_{2} v_{2}}{\rho_{2}}}{a^{2}}\right)
$$

This can be expanded as:

$$
\begin{aligned}
\frac{d\left(\frac{\left(M^{2}-2 M \rho_{2} v_{2}+\left(\rho_{2} v_{2}\right)^{2}\right)}{\rho_{1}}-\frac{\left(\rho_{2} v_{2}\right)^{2}}{\rho_{2}}\right)}{d x} & =-\frac{d\left(\mu_{w} v_{w}\left(\frac{w_{p}}{k_{p}}+\frac{w_{s}}{k_{s}}\right)\right)}{d x} \\
& -F\left(\frac{\mu_{1} M-\mu_{1}\left(\rho_{2} v_{2}\right)}{\rho_{1}\left(a^{*}\right)^{2}}-\frac{\mu_{2}\left(\rho_{2} v_{2}\right)}{\rho_{2} a^{2}}\right)
\end{aligned}
$$

or

$$
\frac{\left(\rho_{2} v_{2}\right)^{2}}{\rho_{2}}=\frac{\left(\rho_{2} v_{2}\right)^{2}}{\rho_{1}} \frac{\rho_{1}}{\rho_{2}}
$$

Also,

$$
\frac{\mu_{2}\left(\rho_{2} v_{2}\right)}{\rho_{2} a^{2}}=\mu_{2} \frac{\rho_{2} v_{2}}{\rho_{1}\left(a^{*}\right)^{2}} \frac{\rho_{1}}{\rho_{2}}\left(\frac{a^{*}}{a}\right)^{2}
$$

Using the assumption $\left(\frac{a^{*}}{a}\right)^{2} \approx 1$ (valid only for PM cake layer thicknesses corresponding to low-moderate PM loading levels in the CPF) in equation (B.21) and substituting in equation (B.19), equation (B.19) can be re-written as:

$$
\begin{aligned}
& \frac{d}{d x}\left(\frac{M^{2}-2 M\left(\rho_{2} v_{2}\right)+\left(\rho_{2} v_{2}\right)^{2}-\frac{\rho_{1}}{\rho_{2}}\left(\rho_{2} v_{2}\right)^{2}}{\rho_{1}}\right) \\
& =-\frac{d}{d x}\left(\mu_{w} v_{w}\left(\frac{w_{p}}{k_{p}}+\frac{w_{s}}{k_{s}}\right)\right) \\
& -F\left(\frac{\mu_{1} M-\mu_{1}\left(\rho_{2} v_{2}\right)-\mu_{2}\left(\rho_{2} v_{2}\right) \frac{\rho_{1}}{\rho_{2}}}{\rho_{1}\left(a^{*}\right)^{2}}\right)
\end{aligned}
$$


We know from quotient rule of differentiation that:

$$
\frac{d}{d x}\left(\frac{f}{g}\right)=\frac{g \frac{d f}{d x}-f \frac{d g}{d x}}{g^{2}}
$$

So, if $K_{1}=M^{2}-2 M\left(\rho_{2} v_{2}\right)+\left(1-\frac{\rho_{1}}{\rho_{2}}\right)\left(\rho_{2} v_{2}\right)^{2}$, we can re-write:

$$
\frac{d}{d x}\left(\frac{K_{1}}{\rho_{1}}\right)=\frac{\rho_{1} \frac{d K_{1}}{d x}-K_{1} \frac{d \rho_{1}}{d x}}{\rho_{1}^{2}}=\frac{1}{\rho_{1}} \frac{d K_{1}}{d x}-\frac{K_{1}}{\rho_{1}^{2}} \frac{d \rho_{1}}{d x}
$$

Also, we can simplify $\frac{d K_{1}}{d x}$ as:

$$
\begin{aligned}
\frac{d K_{1}}{d x} & =\frac{d}{d x}\left(M^{2}-2 M \rho_{2} v_{2}+\left(1-\frac{\rho_{1}}{\rho_{2}}\right)\left(\rho_{2} v_{2}\right)^{2}\right) \\
& =0-2 M \frac{d\left(\rho_{2} v_{2}\right)}{d x}+\left(1-\frac{\rho_{1}}{\rho_{2}}\right) 2\left(\rho_{2} v_{2}\right) \frac{d\left(\rho_{2} v_{2}\right)}{d x}+\left(\rho_{2} v_{2}\right)^{2} \frac{d\left(1-\frac{\rho_{1}}{\rho_{2}}\right)}{d x}
\end{aligned}
$$

Equations (B.24) and (B.25) can be substituted in Equation (B.22) to obtain:

$$
\begin{aligned}
& \frac{1}{\rho_{1}}\left(-2 M \frac{d\left(\rho_{2} v_{2}\right)}{d x}+\left(1-\frac{\rho_{1}}{\rho_{2}}\right) 2\left(\rho_{2} v_{2}\right) \frac{d\left(\rho_{2} v_{2}\right)}{d x}+\left(\rho_{2} v_{2}\right)^{2} \frac{d\left(1-\frac{\rho_{1}}{\rho_{2}}\right)}{d x}\right) \\
& -\frac{M^{2}-2 M\left(\rho_{2} v_{2}\right)+\left(1-\frac{\rho_{1}}{\rho_{2}}\right)\left(\rho_{2} v_{2}\right)^{2}}{\frac{\rho_{1}^{2}}{d x}} \\
& =-\frac{d}{d x}\left(\mu_{w} v_{w}\left(\frac{w_{p}}{k_{p}}+\frac{w_{s}}{k_{s}}\right)\right) \\
& -\frac{F}{\rho_{1}\left(a^{*}\right)^{2}}\left(\mu_{1} M-\left(\mu_{1}+\frac{\rho_{1}}{\rho_{2}} \mu_{2}\right)\left(\rho_{2} v_{2}\right)\right)
\end{aligned}
$$

Now $\rho_{2} v_{2}=N$ and $\frac{\rho_{1}}{\rho_{2}}=H$ can be substituted into Equation (B.26) to obtain:

$$
\begin{aligned}
& \frac{1}{\rho_{1}}\left(-2 M \frac{d N}{d x}+(1-H) 2 N \frac{d N}{d x}+N^{2} \frac{d(1-H)}{d x}\right) \\
& -\frac{M^{2}-2 M N+(1-H) N^{2}}{\rho_{1}^{2}} \frac{d \rho_{1}}{d x} \\
& =-\frac{d\left(\mu_{w} v_{w}\left(\frac{w_{p}}{k_{p}}+\frac{w_{s}}{k_{s}}\right)\right)}{d x} \\
& -\frac{F}{\rho_{1}\left(a^{*}\right)^{2}}\left(\mu_{1} M-\left(\mu_{1}+H \mu_{2}\right) N\right)
\end{aligned}
$$


Also, from Equation (B.1a) and Equation (B.3),

$$
\begin{aligned}
\frac{d}{d x}\left(\mu_{w} v_{w}\left(\frac{w_{p}}{k_{p}}+\frac{w_{s}}{k_{s}}\right)\right) & =\frac{d}{d x}\left(\frac{\mu_{w}}{\rho_{w}} \rho_{w} v_{w}\left(\frac{w_{p}}{k_{p}}+\frac{w_{s}}{k_{s}}\right)\right) \\
& =\frac{d}{d x}\left(\left(\rho_{w} v_{w}\right)\left(\frac{\mu_{w}}{\rho_{w}}\left(\frac{w_{p}}{k_{p}}+\frac{w_{s}}{k_{s}}\right)\right)\right)
\end{aligned}
$$

Using product rule of differentiation, and substituting $\gamma=\frac{\mu_{w}}{\rho_{w}}\left(\frac{w_{p}}{k_{p}}+\frac{w_{s}}{k_{s}}\right)$, Equation (B.28) can be re-written as:

$$
\begin{aligned}
\frac{d}{d x}\left(\rho_{w} v_{w}\left(\frac{w_{p}}{k_{p}}+\frac{w_{s}}{k_{s}}\right)\right) & =\rho_{w} v_{w} \frac{d \gamma}{d x}+\gamma \frac{d\left(\rho_{w} v_{w}\right)}{d x} \\
& =\frac{a}{4} \frac{d\left(\rho_{2} v_{2}\right)}{d x} \frac{d \gamma}{d x}+\gamma \frac{a}{4} \frac{d^{2}\left(\rho_{2} v_{2}\right)}{d x^{2}} \\
& =\frac{a}{4} \frac{d N}{d x} \frac{d \gamma}{d x}+\frac{a}{4} \gamma \frac{d^{2} N}{d x^{2}}
\end{aligned}
$$

Substitute Equation (B.29) in Equation (B.27) to obtain:

$$
\begin{aligned}
\frac{1}{\rho_{1}} & \left(-2 M \frac{d N}{d x}+(1-H) 2 N \frac{d N}{d x}+N^{2} \frac{d(1-H)}{d x}\right) \\
& -\left(\frac{M^{2}-2 M N+(1-H) N^{2}}{\rho_{1}^{2}}\right) \frac{d \rho_{1}}{d x} \\
& =-\frac{a}{4} \frac{d N}{d x} \frac{d \gamma}{d x}-\frac{a}{4} \gamma \frac{d^{2} N}{d x^{2}}-\frac{F}{\rho_{1}\left(a^{*}\right)^{2}}\left(\mu_{1} M-\left(\mu_{1}+H \mu_{2}\right) N\right)
\end{aligned}
$$

Here, $G=\frac{d N}{d x}$ can be substituted to obtain a coupled system of 2 first order ordinary differential equations such that:

$$
\begin{aligned}
& \frac{1}{\rho_{1}}\left(-2 M G+(1-H) 2 N G-N^{2} \frac{d H}{d x}\right) \\
& -\left(\frac{M^{2}-2 M N+(1-H) N^{2}}{\rho_{1}^{2}}\right) \frac{d \rho_{1}}{d x} \\
& =-\frac{a}{4} G \frac{d \gamma}{d x}-\frac{a}{4} \gamma \frac{d G}{d x}-\frac{F}{\rho_{1}\left(a^{*}\right)^{2}}\left(\mu_{1} M-\left(\mu_{1}+H \mu_{2}\right) N\right)
\end{aligned}
$$


Re-arranging,

$$
\begin{aligned}
\left(-\frac{a}{4} \gamma\right) \frac{d G}{d x} & =-\frac{2 M}{\rho_{1}} G+\frac{2 N(1-H)}{\rho_{1}} G-\frac{N^{2}}{\rho_{1}} \frac{d H}{d x}-\frac{M^{2}}{\rho_{1}^{2}} \frac{d \rho_{1}}{d x} \\
& +\frac{2 M N}{\rho_{1}^{2}} \frac{d \rho_{1}}{d x}-\frac{(1-H) N^{2}}{\rho_{1}^{2}} \frac{d \rho_{1}}{d x}+\frac{a}{4} \frac{d \gamma}{d x} G \\
& +\frac{F \mu_{1} M}{\rho_{1}\left(a^{*}\right)^{2}}-\frac{F\left(\mu_{1}+H \mu_{2}\right) N}{\rho_{1}\left(a^{*}\right)^{2}}
\end{aligned}
$$

or

$$
\begin{aligned}
\frac{d G}{d x}=\frac{1}{\left(-\frac{a}{4} \gamma\right)} & {\left[G\left(-\frac{2 M}{\rho_{1}}+\frac{2 N(1-H)}{\rho_{1}}+\frac{a}{4} \frac{d \gamma}{d x}\right)\right.} \\
& +N^{2}\left(-\frac{1}{\rho_{1}} \frac{d H}{d x}-\frac{1-H}{\rho_{1}^{2}} \frac{d \rho_{1}}{d x}\right)+N\left(\frac{2 M}{\rho_{1}^{2}} \frac{d \rho_{1}}{d x}-\frac{F\left(\mu_{1}+H \mu_{2}\right)}{\rho_{1}\left(a^{*}\right)^{2}}\right) \\
& \left.+M^{2}\left(-\frac{1}{\rho_{1}^{2}} \frac{d \rho_{1}}{d x}\right)+M\left(\frac{F \mu_{1}}{\rho_{1}\left(a^{*}\right)^{2}}\right)\right]
\end{aligned}
$$

The system of equations to be solved simultaneously can be re-written as:

$$
\begin{aligned}
\frac{d N}{d x} & =G \\
\frac{d G}{d x} & =\frac{1}{A_{1}}\left[G\left(A_{2}+A_{3}+A_{4}\right)\right. \\
& +N^{2}\left(A_{5}+A_{6}\right) \\
& +N\left(A_{7}+A_{8}\right) \\
& +M^{2}\left(A_{9}\right) \\
& \left.+M\left(A_{10}\right)\right]
\end{aligned}
$$


where:

$$
\begin{aligned}
A_{1} & =-\frac{a}{4} \gamma \\
A_{2} & =-\frac{2 M}{\rho_{1}} \\
A_{3} & =\frac{2 N(1-H)}{\rho_{1}} \\
A_{4} & =\frac{a}{4} \frac{d \gamma}{d x} \\
A_{5} & =-\frac{1}{\rho_{1}} \frac{d H}{d x} \\
A_{6} & =-\frac{1-H}{\rho_{1}^{2}} \frac{d \rho_{1}}{d x} \\
A_{7} & =\frac{2 M}{\rho_{1}^{2}} \frac{d \rho_{1}}{d x} \\
A_{8} & =-\frac{F\left(\mu_{1}+H \mu_{2}\right)}{\rho_{1}\left(a^{*}\right)^{2}} \\
A_{9} & =-\frac{1}{\rho_{1}^{2} \frac{d \rho_{1}}{d x}} \\
A_{10} & =\frac{F \mu_{1}}{\rho_{1}\left(a^{*}\right)^{2}}
\end{aligned}
$$

and

$$
\begin{aligned}
\gamma & =\frac{\mu_{w}}{\rho_{w}}\left(\frac{w_{p}}{k_{p}}+\frac{w_{s}}{k_{s}}\right) \\
H & =\frac{\rho_{1}}{\rho_{2}} \\
M & =\frac{\dot{m}}{n_{\text {cells }} a^{2}} \\
N & =\rho_{2} v_{2}
\end{aligned}
$$




\section{APPENDIX C. DERIVATION OF CONSERVATION OF ENERGY EQUATIONS}

\section{Governing Equations Used}

Energy balance of each control volume is used to develop the differential governing equation which is then solved to obtain the absolute temperature of the exhaust gas mixture in the control volume(s). The time-dependent energy balance of any open control volume can be written as[76]:

$$
\frac{d}{d t}\left(\hat{u}+\frac{v^{2}}{2}+g z\right) \rho V=\dot{Q}-\dot{W}+\sum_{\text {in }} \dot{m}\left(\hat{h}+\frac{v^{2}}{2}+g z\right)-\sum_{\text {out }} \dot{m}\left(\hat{h}+\frac{v^{2}}{2}+g z\right)
$$

where $V$ is the volume of the control volume considered, $\dot{m}$ is the mass flow rate into/out of the control volume, $\hat{u}$ is the specific internal energy of the fluid in the control volume, $\hat{h}$ is the specific enthalpy of the fluid in the control volume, $v$ is the velocity of the fluid in the control volume, $g$ is the acceleration due to gravity, $z$ is the height of the control volume from a reference point, $\dot{Q}$ is the heat transfer rate to/from the control volume across the control surfaces of the control volume and $\dot{W}$ is the rate of work done on/by the system.

This equation can now be applied to specific control volumes and applying certain assumptions that make the treatment simpler.

\section{Inlet Channel}

Figure C.1 shows a schematic representation of a control volume in the inlet channel of the particulate filter. Here, convection of energy has four components. They are:

1. Axial convection through the left face which is located at axial location $x$,

2. Axial convection through the right face which is located at axial location $x+\Delta x$, 
3. Wall convection through the upper, lower, front and back faces, and

4. Heat transfer between inlet channel gas and substrate wall via convection through the upper, lower, front and back faces.

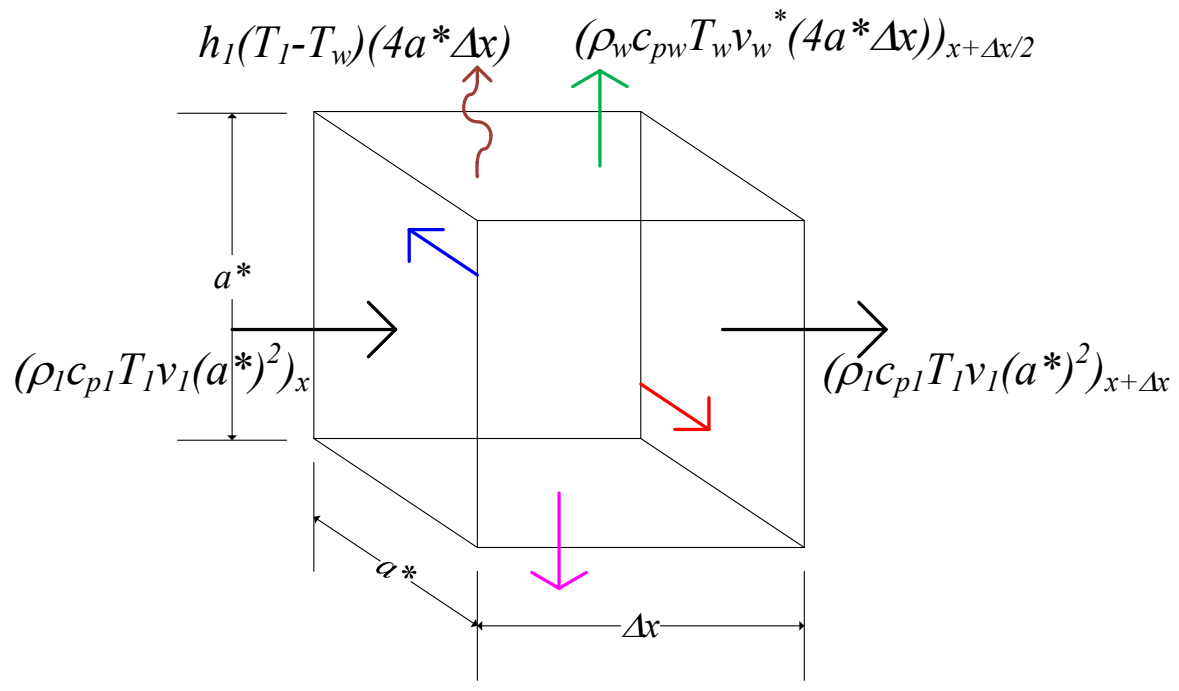

Figure C.1: Schematic representation of a control volume in the inlet channel of the CPF

The transient form of energy balance of the inlet channel CV can be written (from equation (C.1)) as:

$$
\begin{aligned}
\frac{\partial}{\partial t}\left(\rho_{1} c_{v 1} T_{1}\right)\left(a^{*}\right)^{2} \Delta x+\frac{\partial}{\partial x}\left(\rho_{1} v_{1} c_{p 1} T_{1}\right)\left(a^{*}\right)^{2}= & -\left(4 a^{*} \Delta x\right) h_{1}\left(T_{1}-T_{w}\right) \\
& -\left(4 a^{*} \Delta x\right) \rho_{w} v_{w}^{*} c_{p w} T_{w}
\end{aligned}
$$

Assuming quasi-steady state, and dividing all terms by $\left(a^{*}\right)^{2} \Delta x$, equation (C.2) can be re-written as:

$$
\frac{\partial}{\partial x}\left(\rho_{1} v_{1} c_{p 1} T_{1}\right)=-\frac{4}{a^{*}} h_{1}\left(T_{1}-T_{w}\right)-\frac{4}{a^{*}} \rho_{w} v_{w}^{*} c_{p w} T_{w}
$$

Using mass conservation and the assumption that $c_{p 1}$ can be considered as constant, equation (C.3) can be rewritten as:

$$
\frac{\partial T_{1}}{\partial x}=-\frac{4}{a} \frac{a}{a^{*}} \frac{h_{1}}{\rho_{1} v_{1} c_{p 1}}\left(T_{1}-T_{w}\right)
$$




\section{Outlet Channel}

Figure C.2 shows a schematic representation of a control volume in the outlet channel of the particulate filter. Here, convection of energy has four components. They are:

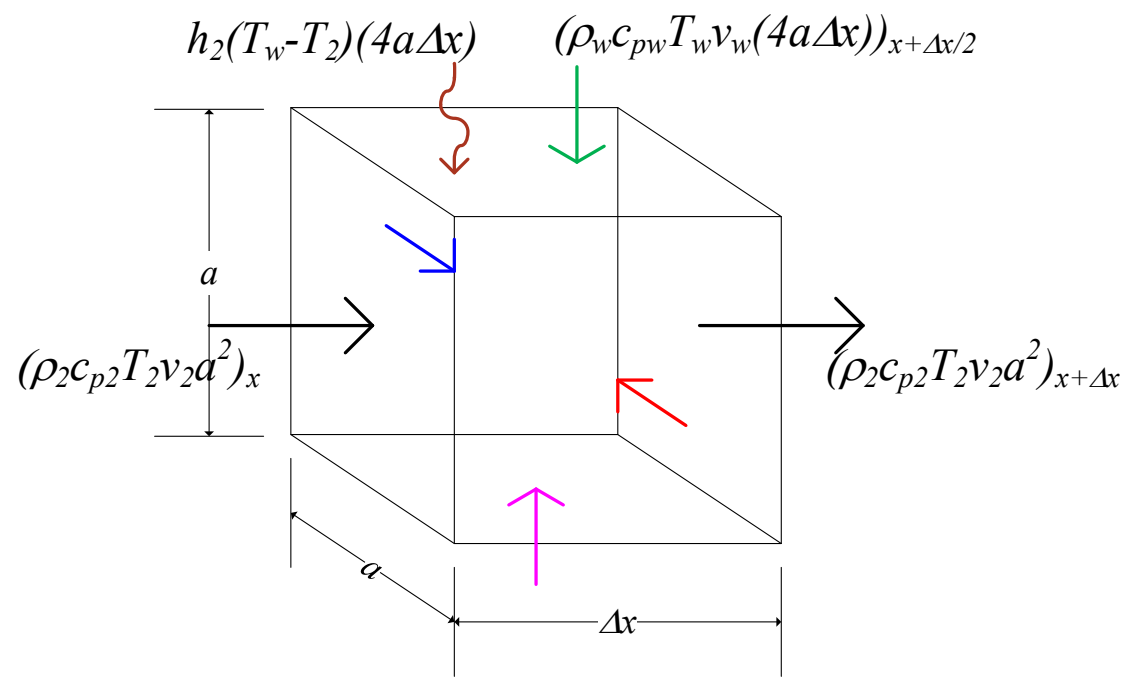

Figure C.2: Schematic representation of a control volume in the outlet channel of the CPF

Transient energy balance of the outlet channel CV can be written (from equation (C.1)) as:

$$
\begin{aligned}
\frac{\partial}{\partial t}\left(\rho_{2} c_{v 2} T_{2}\right) a^{2} \Delta x+\frac{\partial}{\partial x}\left(\rho_{2} v_{2} c_{p 2} T_{2}\right) a^{2}= & -(4 a \Delta x) h_{2}\left(T_{2}-T_{w}\right) \\
& +(4 a \Delta x) \rho_{w} v_{w} c_{p w} T_{w}
\end{aligned}
$$

Assuming quasi-steady state, and dividing all terms by $a^{2} \Delta x$, equation (C.5) can be re-written as:

$$
\frac{\partial}{\partial x}\left(\rho_{2} v_{2} c_{p 2} T_{2}\right)=-\frac{4}{a} h_{2}\left(T_{2}-T_{w}\right)+\frac{4}{a} \rho_{w} v_{w} c_{p w} T_{w}
$$

Using mass conservation and the assumption that $c_{p 2}$ can be considered as constant, equation (C.6) can be rewritten as:

$$
\frac{\partial T_{2}}{\partial x}=-\frac{4}{a} \frac{h_{2}}{\rho_{2} v_{2} c_{p 2}}\left(T_{2}-T_{w}\right)
$$




\section{APPENDIX D. DERIVATION OF GASEOUS SPECIES Concentrations Field}

\section{CPF Species Concentrations Balance Equations}

For each chemical species that form part of the exhaust gas mixture, a balance equation can be written out such that the mass of that particular chemical species is conserved in the control volume(s) considered. All chemical reactions occurring within the CPF as the exhaust gas passes through the different locations of the substrate can be considered to be occurring instantaneously since the chemical reactions occur at a much faster rate than the rate at which the input conditions to the CPF change, the most important of which are the temperature of the inlet gas and that of the substrate itself, and hence the chemical species balance equations can be considered to be having a quasi-steady form, i.e., no temporal gradient terms need be considered. This simplifies the solution procedure since the resulting differential equations are ordinary differential equations (instead of partial differential equations in case temporal gradients are present in addition to spatial (transverse) gradients).

Figure D.1 shows a schematic representation of a discretized element considered which consists of PM cake, catalyst washcoat and substrate wall. This is for a specific case where catalyst washcoat is present as a separate layer in the inlet channel side on the surface of the substrate wall. In case the catalyst washcoat is impregnated into the pores of the substrate wall, the catalyst washcoat layer as shown in Figure D.1 can be specified to be of zero thickness, in which case all gaseous species oxidation reactions (namely that of $\mathrm{HC}, \mathrm{CO}$ and $\mathrm{NO} / \mathrm{NO}_{2}$ ) are assumed to be taking place in the substrate wall sub-domain throughout the entire thickness of the substrate wall.

The entire control volume can be discretized with the help of three user-defined input parameters:

1. $n_{\text {div,cake }}$, the number of divisions in the PM cake layer, 


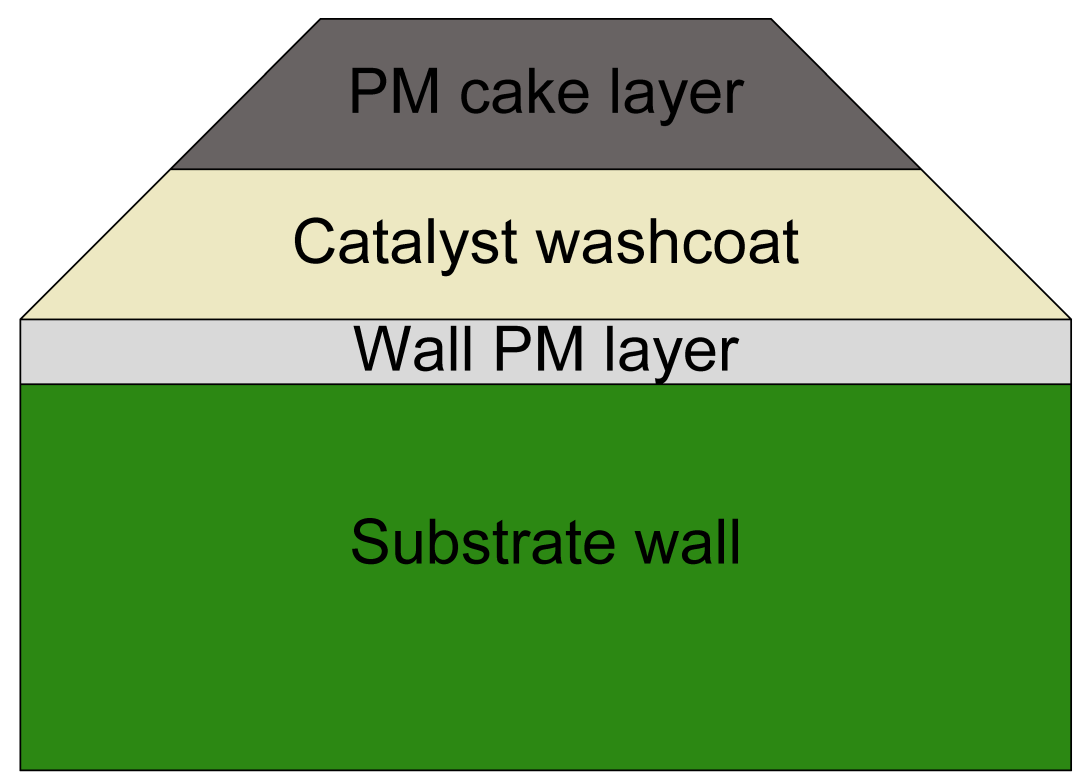

Figure D.1: Schematic representation of the PM cake, catalyst washcoat and substrate wall control volume

2. $n_{\text {div,cat }}$, the number of divisions in the catalyst washcoat, and

3. $n_{\text {div, wall }}$, the number of divisions in the substrate wall

Figure D.2 shows a sample discretization used to sub-divide the PM cake, catalyst washcoat and substrate wall further into smaller discretized slabs in the $(y)$ direction. The black circles denote the nodes at the boundaries $B_{1}$ and $B_{2}$ and all interior points are shown as blue circles.

Figure D. 3 shows a schematic representation of an interior node (intertior nodes are all nodes except the first and last nodes of the control volume) and the control volume enclosing the node.

For all interior points, the components of the mass rates due to different phenomena can be listed as follows:

Rate of convection of species ' $i$ ' at face ' $m-\frac{1}{2}$ ' $=\left.C_{i} M W_{i} a \Delta x v_{w}\right|_{m-\frac{1}{2}}$

Rate of convection of species ' $i$ ' at face ' $m+\frac{1}{2}$ ' $=-\left.C_{i} M W_{i} a \Delta x v_{w}\right|_{m+\frac{1}{2}}$

Rate of diffusion of species ' $i$ ' at face ' $m-\frac{1}{2}$ ' $=-\left.D_{i} M W_{i} \frac{\partial C_{i}}{\partial y} a \Delta x\right|_{m-\frac{1}{2}}$

Rate of diffusion of species ' $i$ ' at face ' $m+\frac{1}{2}$ ' $=\left.D_{i} M W_{i} \frac{\partial C_{i}}{\partial y} a \Delta x\right|_{m+\frac{1}{2}}$ 


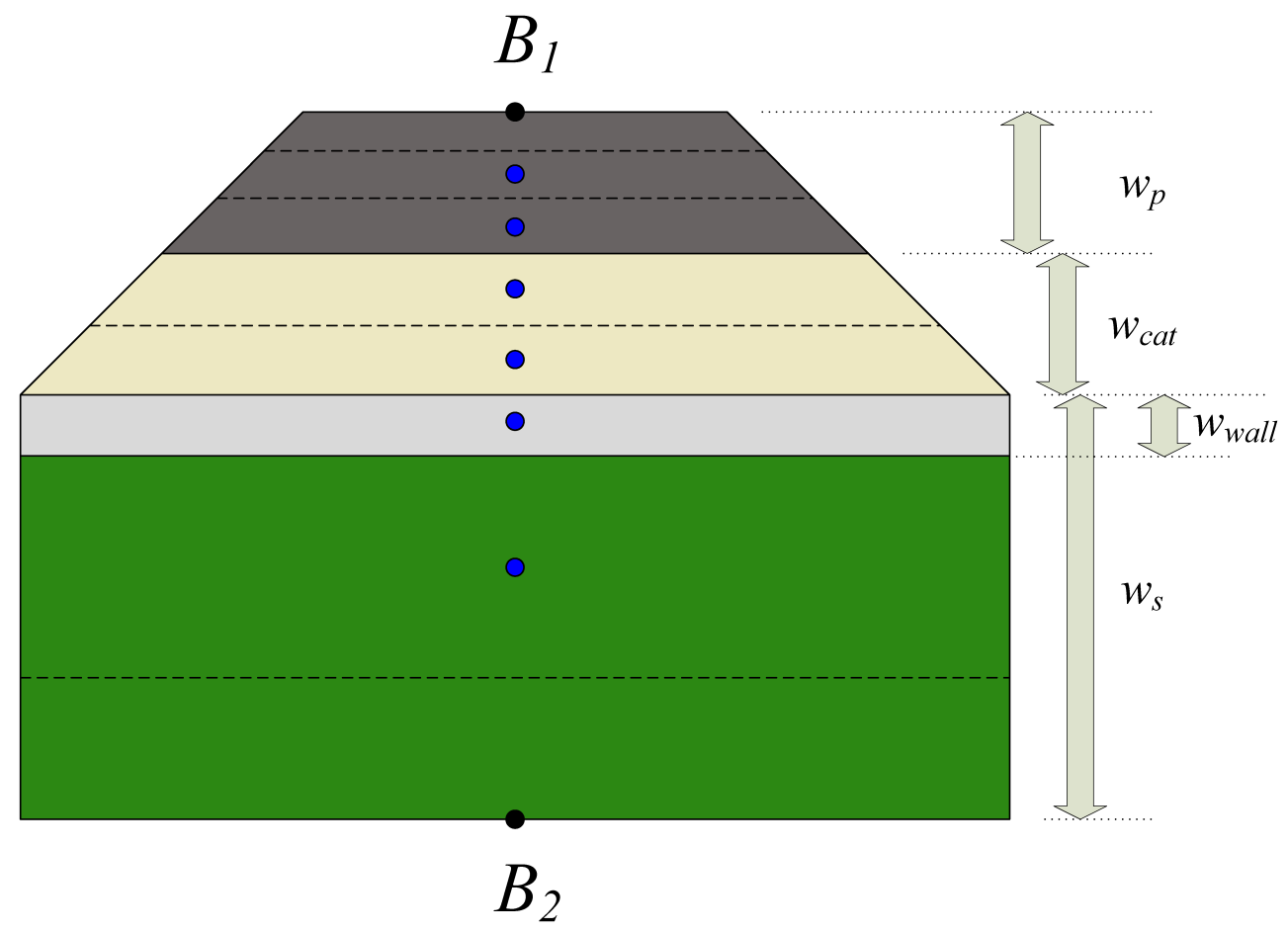

Figure D.2: Sample discretization of the PM cake, catalyst washcoat and substrate wall control volume (Here, $n_{\text {div,cake }}=3, n_{\text {div,cat }}=2$ and $n_{\text {div, wall }}=3$ ). Also shown is the wall PM control volume with the calculated virtual wall layer thickness equal to $w_{\text {wall }}$

Rate of depletion of sp. ' $i$ ' in $\mathrm{CV}=-\sum_{j} \xi_{i j} R_{j} M W_{i}\left(\frac{a_{m-\frac{1}{2}}+a_{m-\frac{1}{2}}}{2}\right)\left(\delta y_{m^{-}}+\delta y_{m^{+}}\right) \Delta x$

Hence, the discretized form of the quasi-steady state species conservation equation can be expressed mathematically as (D.1):

$$
\begin{aligned}
\left(\frac{d m_{i}}{d t}\right)_{c v} & \left.\left.=C_{i} M W_{i} a \Delta x v_{w}\right]_{m-\frac{1}{2}}-C_{i} M W_{i} a \Delta x v_{w}\right]_{m+\frac{1}{2}} \\
& \left.\left.-D_{i} M W_{i} \frac{\partial C_{i}}{\partial y} a \Delta x\right]_{m-\frac{1}{2}}+D_{i} M W_{i} \frac{\partial C_{i}}{\partial y} a \Delta x\right]_{m+\frac{1}{2}} \\
& -\sum_{j} \xi_{i j} R_{j} M W_{i}\left(\frac{a_{m-\frac{1}{2}}+a_{m-\frac{1}{2}}}{2}\right)\left(\delta y_{m^{-}}+\delta y_{m^{+}}\right) \Delta x \\
& =0
\end{aligned}
$$

Canceling out the common terms $\Delta x$ and $M W_{i}$ from all terms in equation (D.1) and 


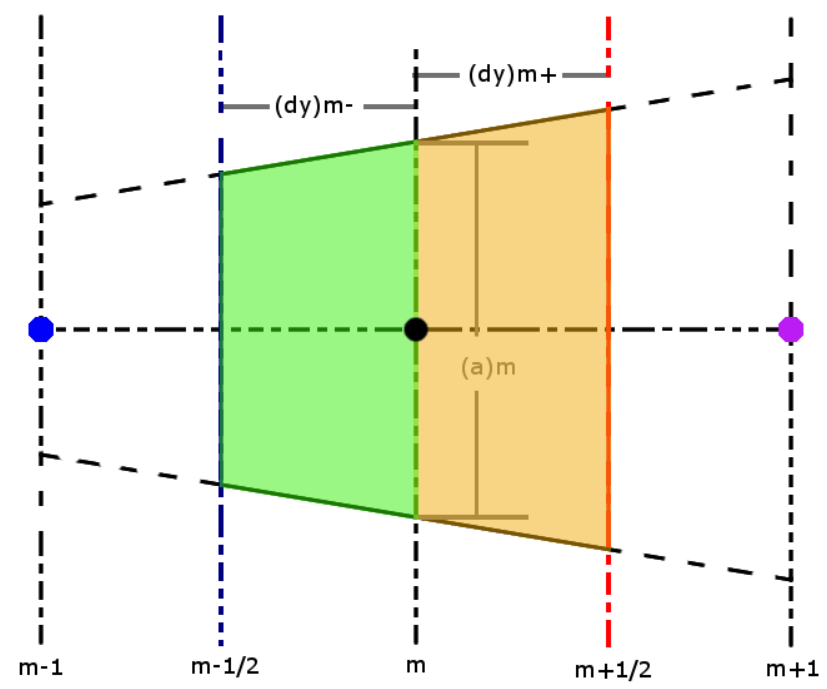

Figure D.3: Schematic representation of an interior node and its neighboring nodes

re-arranging, we get:

$$
\begin{aligned}
\left.\left.C_{i} a v_{w}\right]_{m-\frac{1}{2}}-C_{i} a v_{w}\right]_{m+\frac{1}{2}} & \left.\left.-D_{i} \frac{\partial C_{i}}{\partial y} a\right]_{m-\frac{1}{2}}+D_{i} \frac{\partial C_{i}}{\partial y} a\right]_{m+\frac{1}{2}} \\
& =\sum_{j} \xi_{i j} R_{j}\left(\frac{a_{m-\frac{1}{2}}+a_{m-\frac{1}{2}}}{2}\right)\left(\delta y_{m^{-}}+\delta y_{m^{+}}\right)
\end{aligned}
$$

At this point, two simplifying assumptions are made:

1. Concentration of the mixture is constant throughout the control volume, and

2. Diffusion of chemical species occurs through two mechanisms only, i.e., Brownian (molecular) and Knudsen (pore) types of diffusion.

The constant mixture concentration assumption makes it possible to re-write equation (D.2) in terms of mole fractions ( since $C_{i}=Y_{i} C_{m i x}$ ) as: 


$$
\begin{aligned}
\left.Y_{i} a v_{w}\right|_{m-\frac{1}{2}}-\left.Y_{i} a v_{w}\right|_{m+\frac{1}{2}} & -\left.D_{i} \frac{\partial Y_{i}}{\partial y} a\right|_{m-\frac{1}{2}}+\left.D_{i} \frac{\partial Y_{i}}{\partial y} a\right|_{m+\frac{1}{2}} \\
& =\frac{\Sigma_{j} \xi_{i j} R_{j}}{C_{m i x}}\left(\frac{a_{m-\frac{1}{2}}+a_{m-\frac{1}{2}}}{2}\right)\left(\delta y_{m^{-}}+\delta y_{m^{+}}\right)
\end{aligned}
$$

or in differential form,

$$
a v_{w} \frac{d Y_{i}}{d y}-\frac{d}{d y}\left(D_{i} a \frac{d Y_{i}}{d y}\right)=-\frac{\bar{a}}{C_{m i x}} \sum_{j} \xi_{i j} R_{j}
$$

Since diffusion of chemical species is assumed to occur via two mechanisms, we can compute the effective diffusivity of a particular chemical species knowing the molecular diffusivity, Knudsen diffusivity and microstructural properties of the porous medium that diffusion is occurring in. This can be expressed mathematically as:

$$
D_{e f f, i}=\frac{1}{\frac{\tau}{\epsilon}\left[\frac{1}{D_{m o l, i}}+\frac{1}{D_{K n, i}}\right]}
$$

In equation (D.5), the molecular diffusivity $\left(D_{m o l, i}\right)$ is calculated as follows:

First, binary diffusion coefficients of all gaseous chemical species need to be calculated. This is done according to the method described in [66] as:

$$
D_{i, j}=\frac{a T^{b}}{P}\left(T_{c r i t, i} T_{c r i t, j}\right)^{5 / 12-b / 2}\left(P_{c r i t, i} P_{c r i t, j}\right)^{1 / 3}\left(\frac{1}{M W_{i}}+\frac{1}{M W_{j}}\right)^{1 / 2}
$$

where the binary diffusion coefficients are obtained in the units $\left[\frac{\mathrm{cm}}{\mathrm{s}}\right]$, all temperatures are expressed in $[K]$ and all pressures are expressed in $[\mathrm{atm}$.$] . Knowing the binary$ diffusivities, molecular diffusivities of each chemical species can then be calculated from the Maxwell-Stefan equations for multicomponent diffusion in gases at low density [66] as:

$$
D_{m o l, i}=\frac{1-Y_{i}}{\Sigma_{j \neq i} \frac{Y_{j}}{D_{i, j}}}
$$


The other set of diffusivities needed for computing effective diffusivities are the Knudsen (pore) diffusivities $\left(D_{K n, i}\right)$ of each chemical species. These are calculated according to the method described in reference [77] as:

$$
D_{K n, i}=\frac{d_{p}}{3} \sqrt{\frac{8 R T}{\pi M W_{i}}}
$$

As can be inferred from equation (D.8), the Knudsen diffusivity scales linearly with pore size, linearly with the square root of absolute temperature and inversely with the square root of the molecular weight of the chemical species. Also to be noted is the fact that the Knudsen diffusivities, if they are the dominant set of diffusivities in determining the effective diffusivities, will cause the effective diffusivities of different chemical species to be different by virtue of dependence on molecular weights, even though the diffusion takes place in the same medium at the same conditions of pressure and temperature.

Coming back to equation (D.3), we can make approximations to the interface values from the nodal values of the variable $\left(Y_{i}\right)$ using an upwind scheme for the values used in the convection terms, interface diffusivities calculated so as to preserve interface diffusion fluxes from the method outlined in [68] and a central difference scheme for its gradients $\left(\frac{\partial Y_{i}}{\partial y}\right)$ used in the diffusion terms as follows:

$$
\begin{aligned}
\left(Y_{i}\right)_{m-\frac{1}{2}} & \approx\left(Y_{i}\right)_{m-1} \\
\left(Y_{i}\right)_{m+\frac{1}{2}} & \approx\left(Y_{i}\right)_{m} \\
\left(D_{i}\right)_{m-\frac{1}{2}} & =\left(\frac{1-f_{m}}{\left(D_{i}\right)_{m-1}}+\frac{f_{m}}{\left(D_{i}\right)_{m}}\right)^{-1} \\
\left(D_{i}\right)_{m+\frac{1}{2}} & =\left(\frac{1-g_{m}}{\left(D_{i}\right)_{m}}+\frac{g_{m}}{\left(D_{i}\right)_{m+1}}\right)^{-1} \\
\left(\frac{\partial C_{i}}{\partial y}\right)_{m-\frac{1}{2}} & \approx\left(\frac{\left(Y_{i}\right)_{m}-\left(Y_{i}\right)_{m-1}}{(\delta y)_{m^{-}}+(\delta y)_{m-1^{+}}}\right) \\
\left(\frac{\partial C_{i}}{\partial y}\right)_{m+\frac{1}{2}} & \approx\left(\frac{\left(Y_{i}\right)_{m+1}-\left(Y_{i}\right)_{m}}{(\delta y)_{m+1^{-}}+(\delta y)_{m^{+}}}\right)
\end{aligned}
$$

where: 


$$
\begin{aligned}
f_{m} & =\frac{(\delta y)_{m^{-}}}{(\delta y)_{m^{-}}+(\delta y)_{m-1^{+}}} \\
g_{m} & =\frac{(\delta y)_{m+1^{-}}}{(\delta y)_{m+1^{-}}+(\delta y)_{m^{+}}}
\end{aligned}
$$

For a derivation of effective diffusivities at interfaces from the values of nodal diffusivities, please refer section D.

Substituting equations (D.9) in equation (D.3),

$$
\begin{aligned}
\left(Y_{i}\right)_{m-1}\left(a v_{w}\right)-\left(Y_{i}\right)_{m-1}\left(a v_{w}\right) & -\left(D_{i}\right)_{m-\frac{1}{2}} a_{m-\frac{1}{2}} \frac{\left(Y_{i}\right)_{m}-\left(Y_{i}\right)_{m-1}}{(\delta y)_{m^{-}}+(\delta y)_{m-1^{+}}} \\
& -\left(D_{i}\right)_{m+\frac{1}{2}} a_{m+\frac{1}{2}} \frac{\left(Y_{i}\right)_{m+1}-\left(Y_{i}\right)_{m}}{(\delta y)_{m+1^{-}}+(\delta y)_{m^{+}}} \\
& =\frac{\sum_{j} \xi_{i j} R_{j}}{C_{m i x}}\left(\frac{a_{m-\frac{1}{2}}+a_{m-\frac{1}{2}}}{2}\right)\left(\delta y_{m^{-}}+\delta y_{m^{+}}\right)
\end{aligned}
$$

This can be simplified further by defining some terms:

$$
\begin{aligned}
\phi_{1} & =a v_{w} \\
\phi_{2} & =\frac{\left(D_{i}\right)_{m-\frac{1}{2}} a_{m-\frac{1}{2}}}{(\delta y)_{m^{-}}+(\delta y)_{m-1^{+}}} \\
\phi_{3} & =\frac{\left(D_{i}\right)_{m+\frac{1}{2}} a_{m+\frac{1}{2}}}{(\delta y)_{m+1^{-}}+(\delta y)_{m^{+}}} \\
\phi_{4} & =\frac{\sum_{j} \xi_{i j} R_{j}}{C_{m i x}}\left(\frac{a_{m-\frac{1}{2}}+a_{m-\frac{1}{2}}}{2}\right)\left(\delta y_{m^{-}}+\delta y_{m^{+}}\right)
\end{aligned}
$$

or

$$
\phi_{1}\left(Y_{i}\right)_{m-1}-\phi_{1}\left(Y_{i}\right)_{m}-\phi_{2}\left(Y_{i}\right)_{m}+\phi_{2}\left(Y_{i}\right)_{m-1}+\phi_{3}\left(Y_{i}\right)_{m+1}-\phi_{3}\left(Y_{i}\right)_{m}=\phi_{4}
$$


Re-writing,

$$
\left[\phi_{1}+\phi_{2}\right]\left(Y_{i}\right)_{m-1}+\left[-\phi_{1}-\phi_{2}-\phi_{3}\right]\left(Y_{i}\right)_{m}+\left[\phi_{3}\right]\left(Y_{i}\right)_{m+1}=\phi_{4}
$$

This now is of the tri-diagonal form:

$$
A_{m}\left(Y_{i}\right)_{m-1}+B_{m}\left(Y_{i}\right)_{m}+C_{m}\left(Y_{i}\right)_{m+1}=D_{m}
$$

where

$$
\begin{aligned}
A_{m} & =\phi_{1}+\phi_{2} \\
B_{m} & =-\phi_{1}-\phi_{2}-\phi_{3} \\
C_{m} & =\phi_{3} \\
D_{m} & =\phi_{4}
\end{aligned}
$$

where $\phi_{1}$ thru $\phi_{4}$ are given in equations (D.12a) thru (D.12d) respectively.

For the first point of the control volume which is at boundary ' $B_{1}$ ' (inlet channel - PM cake layer interface), species mole fractions are assumed to be known and equal to the corresponding CPF inlet mole fractions of the species. Mathematically,

$$
\left(Y_{i}\right)_{1}=Y_{i, i n}
$$

Being consistent with a tridiagonal system setup, we can re-write this as:

$$
\text { (0) }\left(Y_{i}\right)_{m-1}+(1)\left(Y_{i}\right)_{m}+(0)\left(Y_{i}\right)_{m+1}=Y_{i, i n}
$$

or

$$
A_{m}\left(Y_{i}\right)_{m-1}+B_{m}\left(Y_{i}\right)_{m}+C_{m}\left(Y_{i}\right)_{m+1}=D_{m}
$$

where 


$$
\begin{aligned}
A_{m} & =0 \\
B_{m} & =1 \\
C_{m} & =0 \\
D_{m} & =Y_{i, \text { in }}
\end{aligned}
$$

where $(m=1)$.

For the last control volume which includes the last point at the boundary ' $B_{2}$ ' of the control volume (i.e., substrate wall - outlet channel interface), the boundary condition is derived from the assumption that the mole fractions of individual chemical species at the 'bottom' of the substrate wall are equal to the corresponding values in the outlet channel can be mathematically expressed as:

$$
\left.\frac{\partial Y_{i}}{\partial y}\right|_{y=+w_{s}}=0
$$

This can be applied to the control volume using a backward difference approximation as:

$$
\left.\frac{\partial Y_{i}}{\partial y}\right|_{y=+w_{s}}=\frac{\left(Y_{i}\right)_{n n}-\left(Y_{i}\right)_{n n-1}}{(\delta y)_{(n n)^{-}}}=0
$$

Re-writing equation (D.22),

$$
\left(Y_{i}\right)_{n n}=\left(Y_{i}\right)_{n n-1}
$$

This can be re-written to be consistent with the tri-diagonal form as:

$$
(-1)\left(Y_{i}\right)_{m-1}+(1)\left(Y_{i}\right)_{m}+(0)\left(Y_{i}\right)_{m+1}=0
$$

or

$$
A_{m}\left(Y_{i}\right)_{m-1}+B_{m}\left(Y_{i}\right)_{m}+C_{m}\left(Y_{i}\right)_{m+1}=D_{m}
$$


where $(m=n n)$ and:

$$
\begin{aligned}
A_{m} & =-1 \\
B_{m} & =1 \\
C_{m} & =0 \\
D_{m} & =0
\end{aligned}
$$

\section{Interface Diffusivities - Derivation}

The values of diffusion coefficients that are used in the finite difference equations used to come up with the tri-diagonal system of equations are not those of the individual nodes which are located in one of three media - PM cake, catalyst wash-coat and substrate wall. Rather, they are the corresponding values at the control surfaces. When this control surface happens to be the interface between two media, the effective diffusivity of the interface should be calculated such that the diffusion flux at the interface is conserved. The CPF catalyst sub-model uses such a calculation which is adapted from a similar calculation as given in reference [68]. The derivation of the same is as follows:

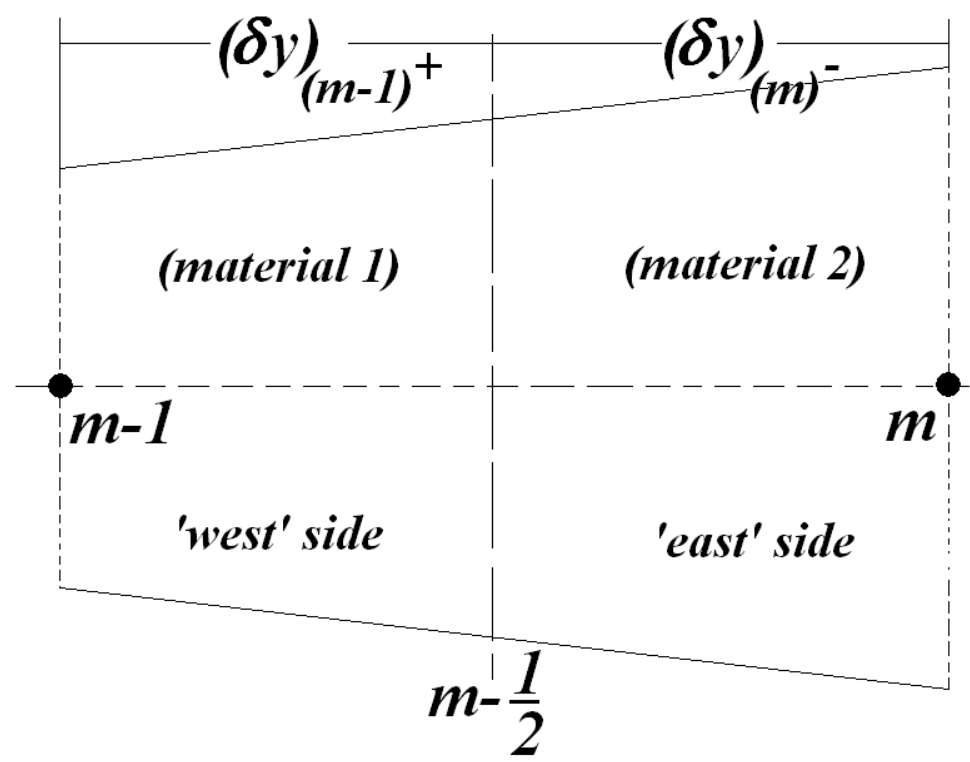

Figure D.4: Schematic representation of an interface shown between two nodes that are in different materials

Diffusion flux of chemical species ' $i$ ' at interface $\left(m-\frac{1}{2}\right)$ can be expressed as: 


$$
q_{i,\left(m-\frac{1}{2}\right)}=-\left(D_{i}\right)_{m-\frac{1}{2}} \frac{\left(Y_{i}\right)_{m}-\left(Y_{i}\right)_{m-1}}{(\delta y)_{(m-1)^{+}}+(\delta y)_{(m)^{-}}}
$$

Similarly, considering the diffusion fluxes on the 'west' $\left(m-\frac{1}{2}\right)^{-}$and 'east' $\left(m-\frac{1}{2}\right)^{+}$ sides of the control surface $\left(m-\frac{1}{2}\right)$, we can write:

$$
\begin{aligned}
& q_{i,\left(m-\frac{1}{2}\right)^{-}}=-\left(D_{i}\right)_{m-1} \frac{\left(Y_{i}\right)_{m-\frac{1}{2}}-\left(Y_{i}\right)_{m-1}}{(\delta y)_{(m-1)^{+}}} \\
& q_{i,\left(m-\frac{1}{2}\right)^{+}}=-\left(D_{i}\right)_{m} \frac{\left(Y_{i}\right)_{m}-\left(Y_{i}\right)_{m-\frac{1}{2}}}{(\delta y)_{(m)^{-}}}
\end{aligned}
$$

From equation (D.28a),

$$
\left(Y_{i}\right)_{m-\frac{1}{2}}-\left(Y_{i}\right)_{m-1}=-q_{i,\left(m-\frac{1}{2}\right)^{-}} \frac{(\delta y)_{(m-1)^{+}}}{\left(D_{i}\right)_{m-1}}
$$

Similarly from equation (D.28b),

$$
\left(Y_{i}\right)_{m}-\left(Y_{i}\right)_{m-\frac{1}{2}}=-q_{i,\left(m-\frac{1}{2}\right)^{+}} \frac{(\delta y)_{(m)^{-}}}{\left(D_{i}\right)_{m}}
$$

Adding equations (D.29) and (D.30), we get:

$$
\left(Y_{i}\right)_{m}-\left(Y_{i}\right)_{m-1}=-q_{i,\left(m-\frac{1}{2}\right)^{-}} \frac{(\delta y)_{(m-1)^{+}}}{\left(D_{i}\right)_{m-1}}-q_{i,\left(m-\frac{1}{2}\right)^{+}} \frac{(\delta y)_{(m)^{-}}}{\left(D_{i}\right)_{m}}
$$

From equation (D.27), we have:

$$
\left(Y_{i}\right)_{m}-\left(Y_{i}\right)_{m-1}=-q_{i,\left(m-\frac{1}{2}\right)} \frac{(\delta y)_{(m-1)^{+}}+(\delta y)_{(m)^{-}}}{-\left(D_{i}\right)_{m-\frac{1}{2}}}
$$

Equating the left-hand sides of equations (D.31) and (D.32), 
$-q_{i,\left(m-\frac{1}{2}\right)^{-}} \frac{(\delta y)_{(m-1)^{+}}}{\left(D_{i}\right)_{m-1}}-q_{i,\left(m-\frac{1}{2}\right)^{+}} \frac{(\delta y)_{(m)^{-}}}{\left(D_{i}\right)_{m}}=-q_{i,\left(m-\frac{1}{2}\right)} \frac{(\delta y)_{(m-1)^{+}}+(\delta y)_{(m)^{-}}}{\left(D_{i}\right)_{m-\frac{1}{2}}}$

But we also know that in order to conserve diffusion flux,

$$
q_{i,\left(m-\frac{1}{2}\right)^{-}}=q_{i,\left(m-\frac{1}{2}\right)^{+}}=q_{i,\left(m-\frac{1}{2}\right)}=\left(q_{i}\right)_{d i f f}
$$

Therefore, equation (D.33) becomes:

$$
\frac{(\delta y)_{(m-1)^{+}}+(\delta y)_{(m)^{-}}}{\left(D_{i}\right)_{m-\frac{1}{2}}}=\frac{(\delta y)_{(m-1)^{+}}}{\left(D_{i}\right)_{m-1}}+\frac{(\delta y)_{(m)^{-}}}{\left(D_{i}\right)_{m}}
$$

or

$$
\left(D_{i}\right)_{m-\frac{1}{2}}=\frac{(\delta y)_{(m-1)^{+}}+(\delta y)_{(m)^{-}}}{\frac{(\delta y)_{(m-1)^{+}}}{\left(D_{i}\right)_{m-1}}+\frac{(\delta y)_{(m)^{-}}}{\left(D_{i}\right)_{m}}}
$$

If we define a ratio $(f)_{m}$ such that:

$$
(f)_{m}=\frac{(\delta y)_{(m)^{-}}}{(\delta y)_{(m-1)^{+}}+(\delta y)_{(m)^{-}}}
$$

Equation (D.37) can then be re-written as:

$$
\left(D_{i}\right)_{m-\frac{1}{2}}=\frac{1}{\frac{1-(f)_{m}}{\left(D_{i}\right)_{m-1}}+\frac{(f)_{m}}{\left(D_{i}\right)_{m}}}
$$




\section{APPENDIX E. FLOWCHARTS FOR THE CPF Model Source Code}




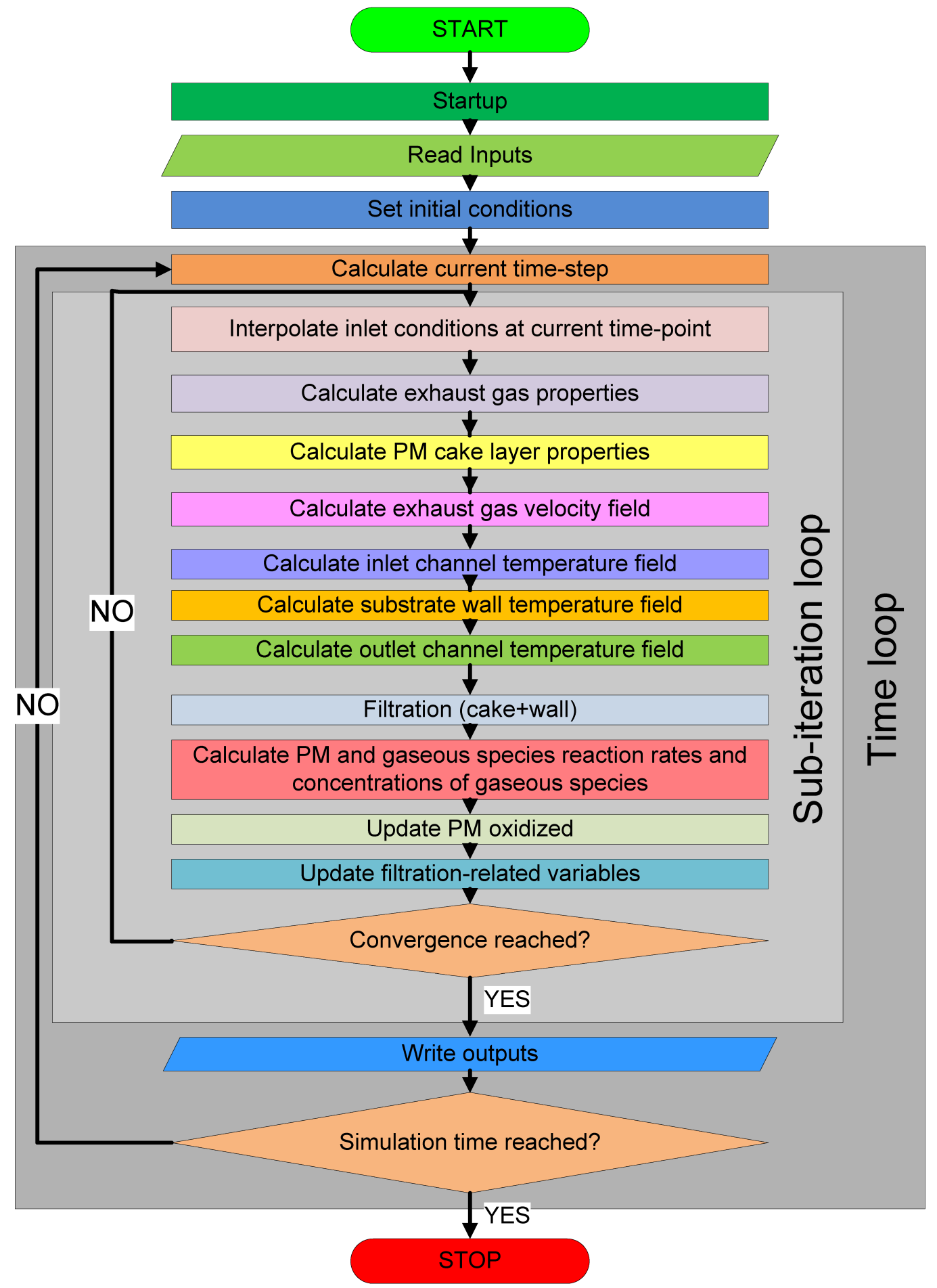

Figure E.1: Flowchart showing a detailed flow of logic in the MTU CPF high-fidelity model version 4.0. Reprinted with permission from SAE Paper No. 2013-01-1574 (c)2013 SAE International. 


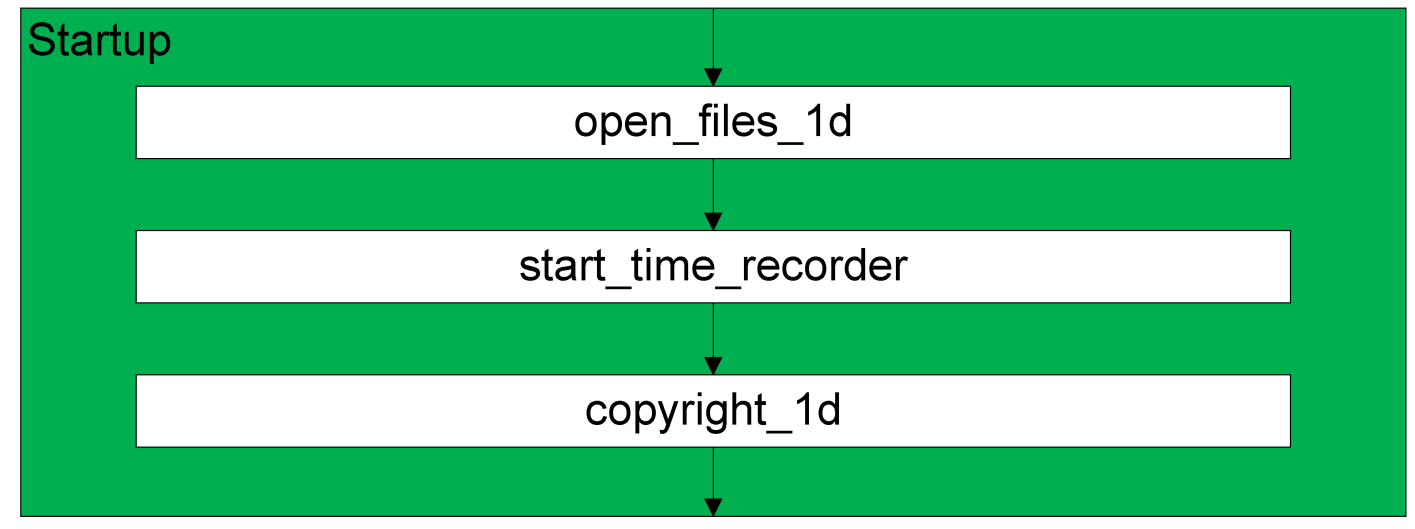

Figure E.2: Flowchart of the Startup subroutine as shown in Figure E.1 


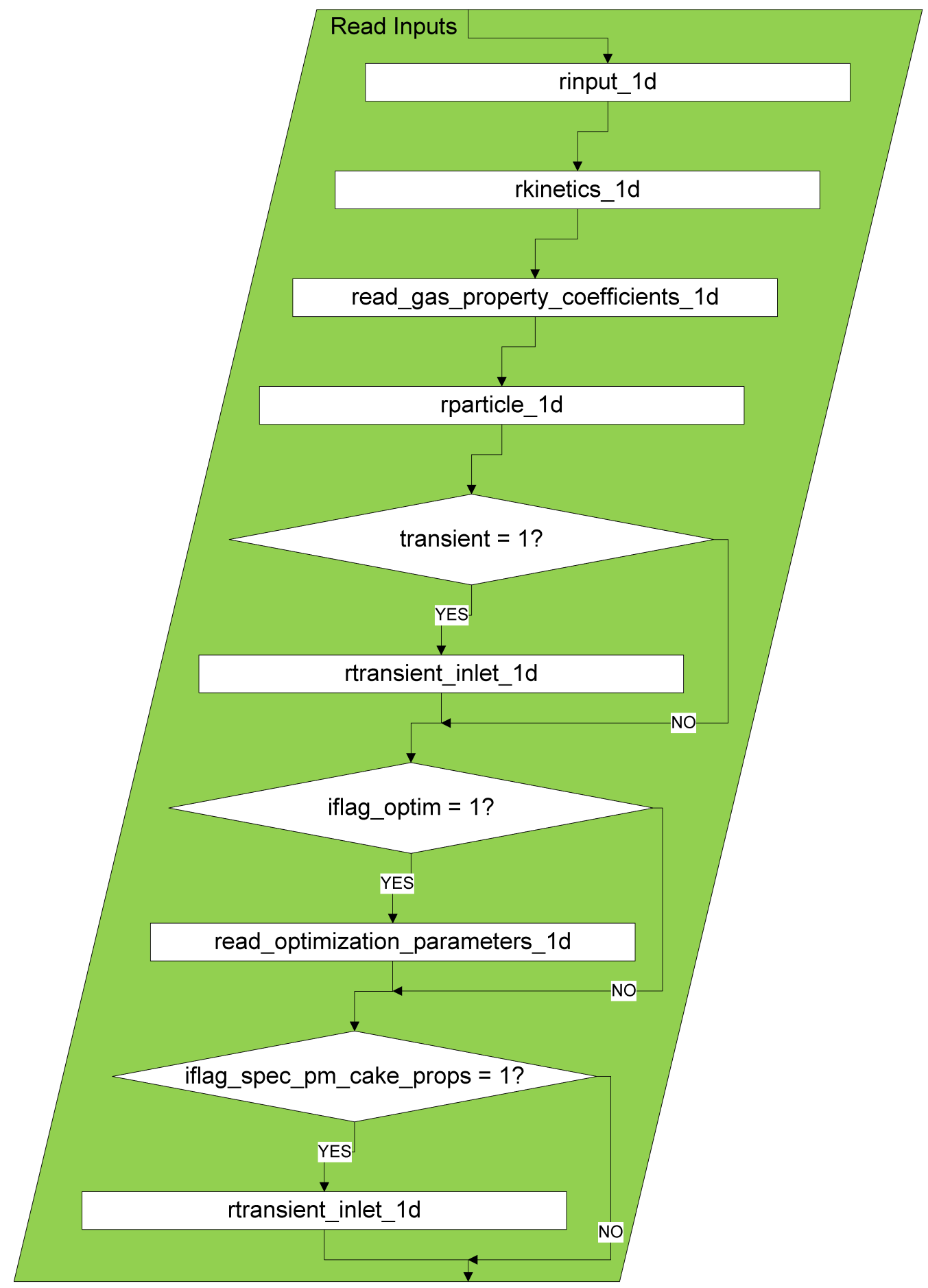

Figure E.3: Flowchart of the Read Inputs subroutine as shown in Figure E.1 

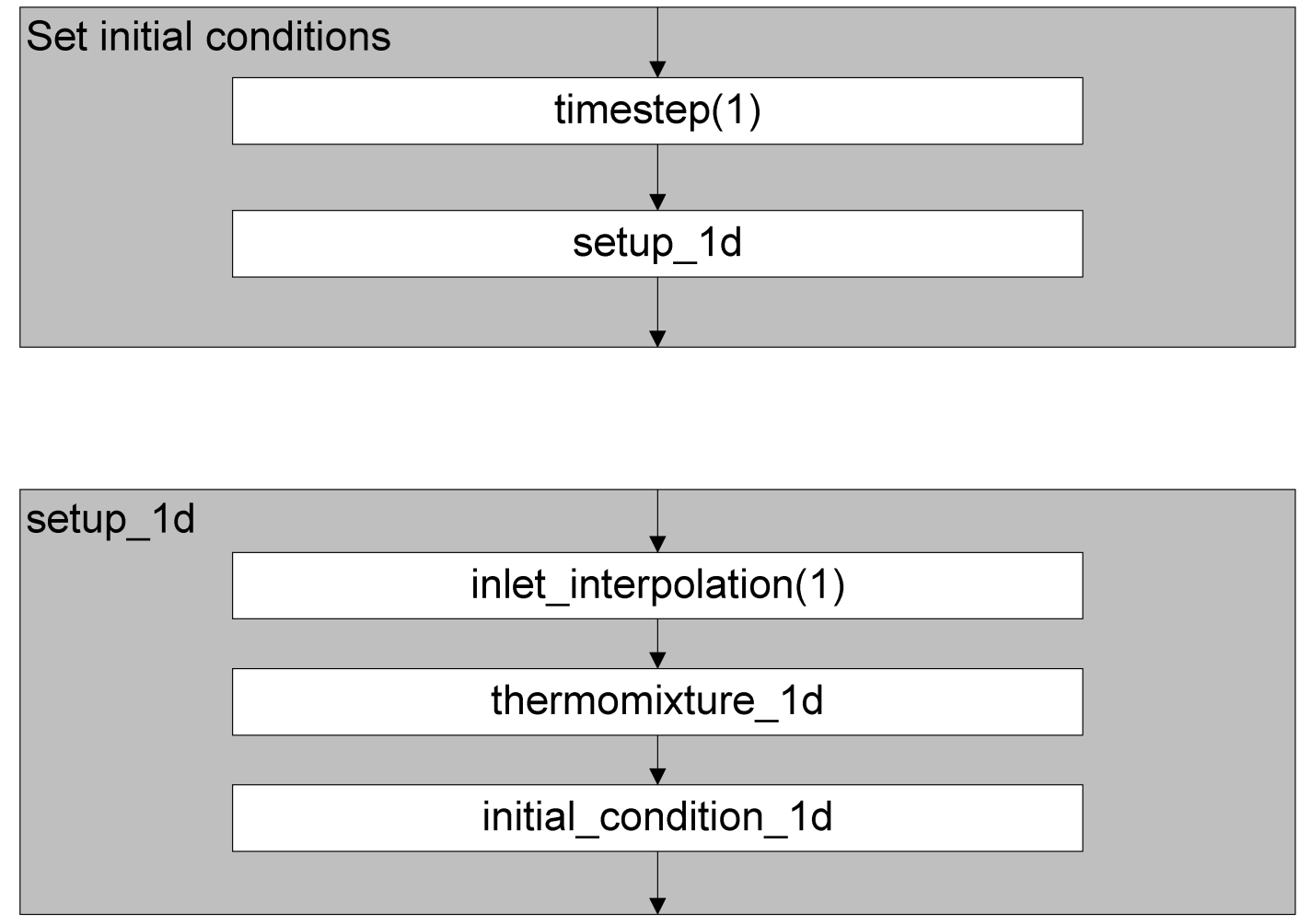

Figure E.4: Flowchart of the Set Initial Conditions subroutine as shown in Figure E.1 


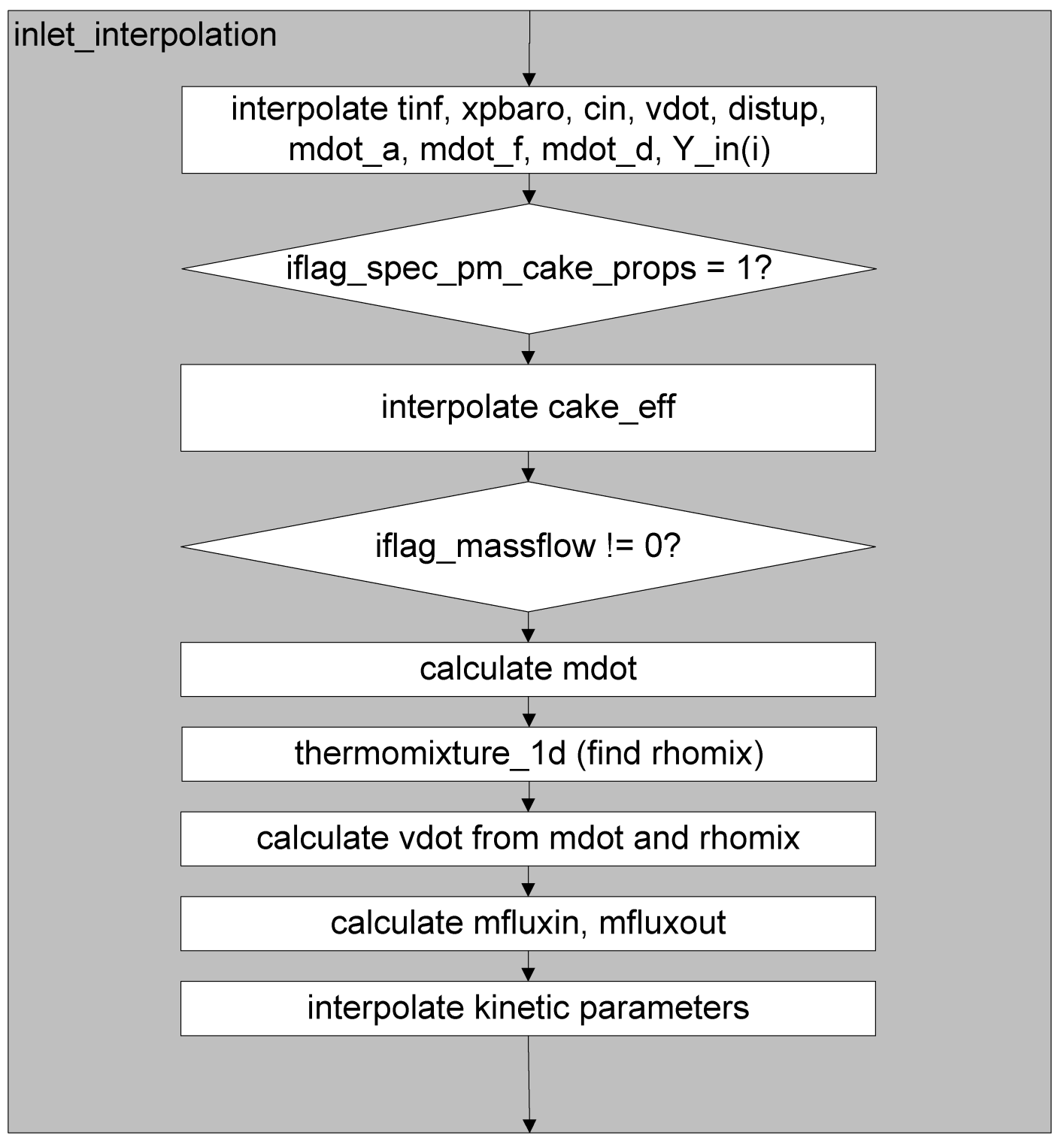

Figure E.5: Flowchart of the Inlet Interpolation subroutine as shown in Figure E.1 
START

\section{t}

Calculate PM inlet mass distribution axially, total CPF inlet PM mass rate, total CPF inlet PM mass

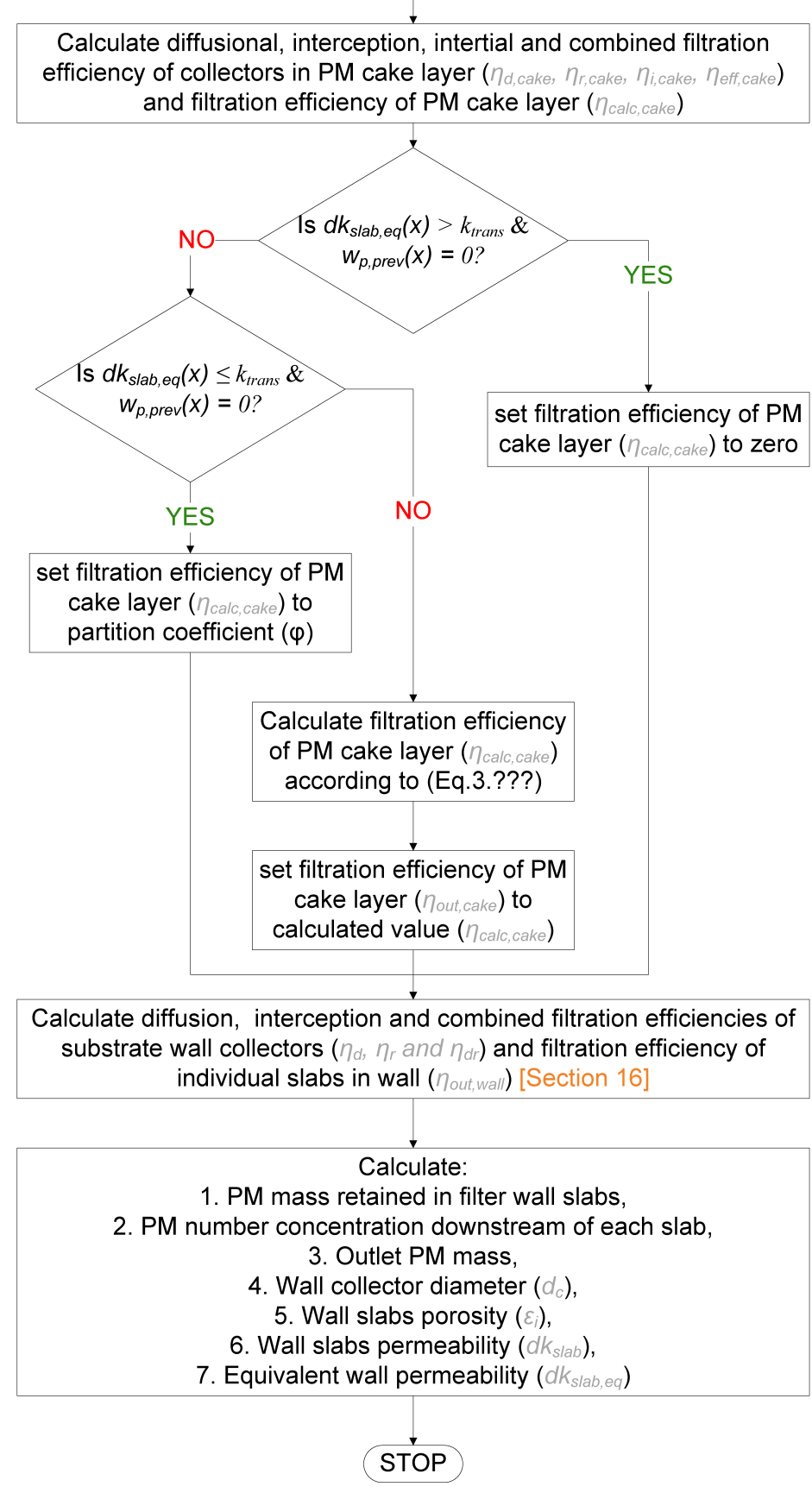

Figure E.6: Flowchart of the filtration subroutine as shown in Figure E.1 - box titled 'Filtration (cake+wall)' 


\section{APPENDIX F. COMPILING AND RUNNING THE CPF Model Source Code}

The MTU 1-D CPF model version 4.0 was developed entirely in the FORTRAN77 programming language. The model code consists of 2 files: the fortran source code file (which has the extension . $f$; hereafter called the '.f file') and the variable declarations file (which has the extension . $i$; hereafter referred to as the '. $i$ file'). In order for a simulation to be performed, the source code file has to be compiled using a FORTRAN compiler (such as gfortran, Intel@Fortran Compiler etc.). In order for the compilation process to be successful, the . f file and the . i file have to be in the same folder as the present working directory from which the compile command is issued. Compilation can be carried out by issuing the compiler command along with desired options at a command prompt. For example, on a windows $\mathrm{PC}$ on which Intel $\mathrm{R}$ Fortran Compiler is installed and available, the user opens a windows command prompt, changes present working directory to the desired folder and issues the command:

ifort/real_size:64 file.f

where $f i l e . f$ is the name of the fortran source code. The option /real_size: 64 directs the compiler to define all REAL type variable declarations, constants, functions, and intrinsics as REAL $* 8$ (DOUBLE PRECISION or KIND = 8), and is required for ensuring accurate results.

Compilation of the source code produces an output file (which has the same name as the Fortran source code by default, and an extension . out on UNIX/Linux systems or . exe on Microsoft Windows-based systems), which is the executable file. This file is hereafter called the '. exe file'.

In the above example, issuing the compile command as given above produces a file named $\mathrm{file}$. exe on a Windows-based system in the present working directory.

The executable file thus created can now be used for running simulations with. Please note that the compilation also produces an 'object file' (which is by default assigned the 
name file.obj; hereafter called the '.obj file') which has to be in the same directory as the .exe file. In addition to this, all input files that the source code opens during the simulation need to be in the same folder as the .exe and . obj files for the simulation to be successfully completed.

\section{Files Needed}

\section{Input Files (*.dat)}

Inputs to the model are specified by ASCII text files, all of which have the extension . dat. This set of input files are as described below:

1. adsorption_parameters_v4_b04.dat,

2. datasave_tsamples_v4_b04.dat,

3. gas_prop_coeff_v4_b04.dat,

4. input_dia_distup_v4_b04.dat,

5. input_exp_tout_solidwall_v4_b04.dat,

6. input_gas_kinetics_v4_b04.dat,

7. input_parameters_v4_b04.dat,

8. input_pm_kinetics_v4_b04.dat, and

9. timepoints_v4_b04.dat

\section{Comparison Files $(* . c m p)$}

Experimental data to be compared with model-predicted data is also stored in text files with extension . cmp. This set of comparison files are:

1. exp_c12h24_concentrations.cmp: Contains experimental data about hydrocarbon measurements made (expressed in terms of $\mathrm{C}_{12} \mathrm{H}_{24}$ by dividing $\mathrm{HC}$ concentrations $[p p m C]$ by 12) during the course of the experiment. It has 3 columns of data and 1 column for comments and description; column 1 contains time points (expressed in $[h r]$ ), column 2 contains UPCPF values of $C_{12} H_{24}$ concentrations 
in parts per million $[\mathrm{ppm}]$ and column 3 contains DNCPF values of $\mathrm{C}_{12} \mathrm{H}_{24}$ concentrations $[p p m]$.

2. exp_co_concentrations.cmp: : Contains experimental data about carbon monoxide concentration measurements made. It has 3 columns of data and 1 column for comments and description; column 1 contains time points $[h r]$, column 2 contains UPCPF $C O$ concentrations $[\mathrm{ppm}]$ and column 3 contains DNCPF $C O$ concentrations $[\mathrm{ppm}]$.

3. exp_no_concentrations.cmp: : Contains experimental data about nitric oxide concentration measurements made. It has 3 columns of data and 1 column for comments and description; column 1 contains time points $[h r]$, column 2 contains UPCPF $N O$ concentrations $[p p m]$ and column 3 contains DNCPF NO concentrations $[\mathrm{ppm}]$.

4. exp_no2_concentrations.cmp: : Contains experimental data about nitrogen dioxide concentration measurements made. It has 3 columns of data and 1 column for comments and description; column 1 contains time points $[h r]$, column 2 contains UPCPF $\mathrm{NO}_{2}$ concentrations $[\mathrm{ppm}]$ and column 3 contains DNCPF $\mathrm{NO}_{2}$ concentrations $[\mathrm{ppm}]$.

5. exp_pdrop . cmp: Contains CPF pressure drop experimental data. It has 2 columns of data; column 1 is time data $[h r]$ and column 2 is corresponding pressure drop data $[k P a]$.

6. exp_pm_retained.cmp: Contains experimental data regarding PM retained measured/calculated. It has 2 columns of data; column 1 is time data $[h r]$ and column 2 is corresponding PM retained data $[g]$.

\section{Output Files $(* . t x t)$}

Model-predicted output is written out in ASCII text format in various output files, all of which have the extension . txt. Some of the important output files are:

1. pressure_drop_components_vs_time.txt,

2. particulate_mass_vs_time.txt,

3. temperature_vs_time.txt,

4. efficiency_vs_time.txt,

5. gaseous_velocity_vs_time.txt, 
6. c12h24_vs_time.txt,

7. co_vs_time.txt,

8. no_vs_time.txt,

9. no2_vs_time.txt,

10. pm_mass_oxid_details_vs_time.txt,

11. cakemass_vs_time.txt, and

12. wallmass_vs_time.txt.

All output files are also written out in the present working directory. These files can be imported into programs that facilitate plotting such as Microsoft Excel or Matlab, and compared and analyzed with the various plotting and comparison tools/methods available in these programs. 


\section{APPENDIX G. Gas Property COEFFICIENTS USED}

Table G.1: Coefficients used in the calculation of specific heat of exhaust gas mixture - see equation (3.4) [66]

\begin{tabular}{|c|l|c|c|c|c|c|}
\hline Index & Species & $A_{i}$ & $B_{i}$ & $C_{i}$ & $D_{i}$ & $E_{i}$ \\
\hline 1 & $\mathrm{C}_{12} \mathrm{H}_{24}$ & $1.87 \mathrm{E}+05$ & $5.83 \mathrm{E}+05$ & $1.56 \mathrm{E}+03$ & $4.24 \mathrm{E}+05$ & $7.21 \mathrm{E}+02$ \\
2 & $\mathrm{O}_{2}$ & $2.91 \mathrm{E}+04$ & $1.00 \mathrm{E}+04$ & $2.53 \mathrm{E}+03$ & $9.36 \mathrm{E}+03$ & $1.15 \mathrm{E}+03$ \\
3 & $\mathrm{~N}_{2}$ & $2.91 \mathrm{E}+04$ & $8.61 \mathrm{E}+03$ & $1.70 \mathrm{E}+03$ & $1.03 \mathrm{E}+02$ & $9.10 \mathrm{E}+02$ \\
4 & $\mathrm{CO}_{2}$ & $2.94 \mathrm{E}+04$ & $3.45 \mathrm{E}+04$ & $-1.43 \mathrm{E}+03$ & $2.64 \mathrm{E}+04$ & $5.88 \mathrm{E}+02$ \\
5 & $\mathrm{H}_{2} \mathrm{O}$ & $3.34 \mathrm{E}+04$ & $2.68 \mathrm{E}+04$ & $2.61 \mathrm{E}+03$ & $8.90 \mathrm{E}+03$ & $1.17 \mathrm{E}+03$ \\
7 & $\mathrm{CO}$ & $2.91 \mathrm{E}+04$ & $8.77 \mathrm{E}+03$ & $3.09 \mathrm{E}+03$ & $8.46 \mathrm{E}+03$ & $1.54 \mathrm{E}+03$ \\
8 & $\mathrm{NO}$ & $3.32 \mathrm{E}+04$ & $-2.36 \mathrm{E}+01$ & $5.32 \mathrm{E}-02$ & $-3.79 \mathrm{E}-05$ & $9.12 \mathrm{E}-09$ \\
9 & $\mathrm{NO}_{2}$ & $3.33 \mathrm{E}+04$ & $2.49 \mathrm{E}+04$ & $1.11 \mathrm{E}+03$ & $9.25 \mathrm{E}+03$ & $5.59 \mathrm{E}+02$ \\
\hline
\end{tabular}

Table G.2: Coefficients used in the calculation of dynamic viscosity of individual chemical species - see equation (3.6) [66]

\begin{tabular}{|c|l|c|c|c|c|}
\hline Index & Species & $A_{i}$ & $B_{i}$ & $C_{i}$ & $D_{i}$ \\
\hline 1 & $\mathrm{C}_{12} \mathrm{H}_{24}$ & $5.24 \mathrm{E}-07$ & $5.07 \mathrm{E}-01$ & $2.69 \mathrm{E}+02$ & 0 \\
2 & $\mathrm{O}_{2}$ & $8.04 \mathrm{E}-07$ & $6.05 \mathrm{E}-01$ & $7.03 \mathrm{E}+01$ & 0 \\
3 & $\mathrm{~N}_{2}$ & $7.63 \mathrm{E}-07$ & $5.88 \mathrm{E}-01$ & $6.78 \mathrm{E}+01$ & 0 \\
4 & $\mathrm{CO}_{2}$ & $2.15 \mathrm{E}-06$ & $4.60 \mathrm{E}-01$ & $2.90 \mathrm{E}+02$ & 0 \\
5 & $\mathrm{H}_{2} \mathrm{O}$ & $6.18 \mathrm{E}-07$ & $6.78 \mathrm{E}-01$ & $8.47 \mathrm{E}+02$ & $-7.39 \mathrm{E}+04$ \\
7 & $\mathrm{CO}$ & $1.11 \mathrm{E}-06$ & $5.34 \mathrm{E}-01$ & $9.47 \mathrm{E}+01$ & 0 \\
8 & $\mathrm{NO}$ & $1.47 \mathrm{E}-06$ & $5.12 \mathrm{E}-01$ & $1.25 \mathrm{E}+02$ & 0 \\
9 & $\mathrm{NO}_{2}$ & $6.13 \mathrm{E}-08$ & $9.22 \mathrm{E}-01$ & $-2.85 \mathrm{E}+02$ & $7.44 \mathrm{E}+04$ \\
\hline
\end{tabular}


Table G.3: Coefficients used in the calculation of thermal conductivity of individual chemical species - see equation (3.10) [66]

\begin{tabular}{|c|l|c|c|c|c|}
\hline Index & Species & $A_{i}$ & $B_{i}$ & $C_{i}$ & $D_{i}$ \\
\hline 1 & $C_{12} H_{24}$ & $9.16 \mathrm{E}-05$ & $1.05 \mathrm{E}+00$ & $7.21 \mathrm{E}+02$ & 0 \\
2 & $\mathrm{O}_{2}$ & $4.94 \mathrm{E}-04$ & $7.34 \mathrm{E}-01$ & $7.00 \mathrm{E}+01$ & 0 \\
3 & $\mathrm{~N}_{2}$ & $3.51 \mathrm{E}-04$ & $7.65 \mathrm{E}-01$ & $2.58 \mathrm{E}+01$ & 0 \\
4 & $\mathrm{CO}_{2}$ & $2.15 \mathrm{E}-06$ & $4.60 \mathrm{E}-01$ & $2.90 \mathrm{E}+02$ & 0 \\
5 & $\mathrm{H}_{2} \mathrm{O}$ & $2.16 \mathrm{E}-03$ & $7.68 \mathrm{E}-01$ & $3.94 \mathrm{E}+03$ & $-4.45 \mathrm{E}+05$ \\
7 & $\mathrm{CO}$ & $8.39 \mathrm{E}-04$ & $6.41 \mathrm{E}-01$ & $8.61 \mathrm{E}+01$ & 0 \\
8 & $\mathrm{NO}$ & $2.41 \mathrm{E}-04$ & $8.27 \mathrm{E}-01$ & $1.70 \mathrm{E}+01$ & 0 \\
9 & $\mathrm{NO}_{2}$ & $7.09 \mathrm{E}-02$ & $-2.14 \mathrm{E}-01$ & $-1.26 \mathrm{E}+03$ & $4.30 \mathrm{E}+05$ \\
\hline
\end{tabular}




\section{APPENDIX H. EXhaust Gas InPUT DATa}

\section{Correction Factor (CF) to Model Input Flow Rates from PO and AR Experiments}

This section has been re-produced with permission (please refer Appendix L) from reference [55]. The correction factor (CF) discussed in reference [55] and shown in Table H. 1 is determined as follows:

First, the $\mathrm{CPF}$ resistance is calculated using the CPF pressure drop corrected for offsets and exhaust mass flow rate corrected with the scaling factors. The CPF resistance for test AR-B20-1 is shown in Figure H.1. Again, the corrections applied to the data in Figure H.1 are only to account for LFE pressure drop measurement error and/or exhaust bypass valve leaks affecting the mass flow rate and pressure drop offset affecting the CPF pressure drop curve.

Next, the graphical input tool within MATLAB is used to re-construct the plausible resistance curve based on the DAQ measurement error corrected CPF pressure drop and exhaust mass flow rates (shown in red on the first subplot in Figure H.1). Using this resistance curve from the graphical input, the required $\mathrm{CF}$ is determined using the $\mathrm{CPF}$ resistance equation. Average values for the corrections (SF) applied to all test phases based on DAQ system measurement errors and/or bypass valve leaks as tabulated in Table $\mathrm{H} 3$ of Reference [55] are used in the analysis. The active regeneration ramp and active regeneration phase correction factor $(\mathrm{CF})$ is determined using the experimental exhaust mass flow rate (after SF from Table H3 of Reference [55] was applied, where required). The plausible resistance curve is used to calculate a correction factor $(\mathrm{CF})$ for the pre-active regeneration ramp and active regeneration test phases. Eqn. 20 of Reference [55] can be re-written to solve for the correction factor, CF, which is shown by Equation H.1.

$$
C F=\frac{\Delta P V_{t} \rho_{e x h}}{\dot{m} \mu R}
$$



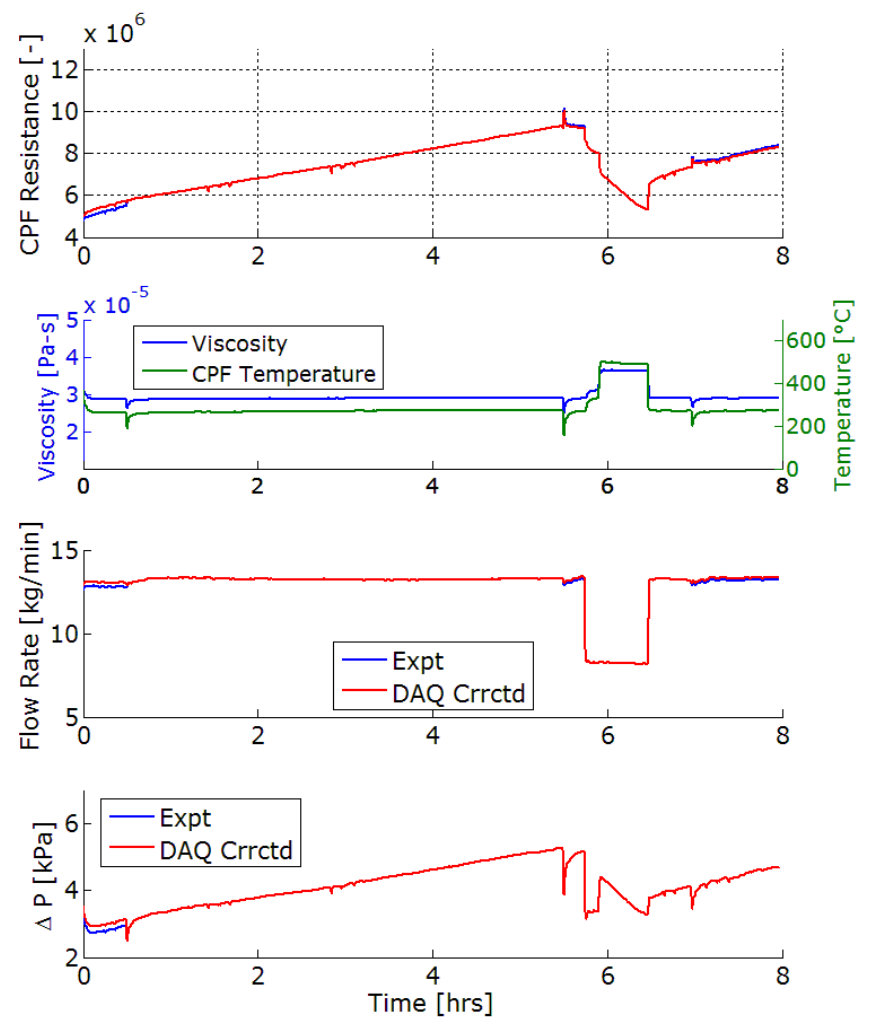

Figure H.1: AR-B20-1 - Corrected and Experimental CPF Flow Resistance (subplot 1), Exhaust Viscosity and CPF Average Temperature (subplot 2), Corrected and Corrected Experimental Exhaust Mass Flow Rate (subplot 3), Corrected and Experimental CPF Pressure Drop Profile (subplot 4)

The variable $R$ in Equation H.1 comes from the re-constructed plausible resistance curve where all other variables used to solve for $C F$ come from the experimental data. The resulting $C F$ is a scalar multiplier to be applied to the experimental mass flow rate in order to simplify input data preparation for the MTU-1D CPF model. The average $C F$ for pre-active regeneration ramp and active regeneration phases for each experiment can be seen in Table H.1.

The resulting $C F$ is equal to 1 in all test phases except active regeneration ramp and active regeneration, because $C F$ corrects for possible temperature and PM maldistribution in the CPF, exhaust flow maldistribution at the CPF inlet, and changing cake layer permeability which cannot be quantified from experimental data at this time. It is believed that there are two triggers which drive the need for the correction factor $(C F)$. The first is the switch to the active regeneration engine condition during the AR-ramp test phase. The second is the initiation of active regeneration by fuel dosing because the $C F$ rapidly changes during the first few minutes of temperature increase after dosing has been initiated 
Table H.1: Correction factors (CF) used for calibration of the CPF model

\begin{tabular}{|c|c|c|c|}
\hline No. & Expt. Name & \multicolumn{2}{|c|}{ Correction Factor (CF) [.] } \\
\hline \multicolumn{4}{|c|}{ Passive Oxidation } \\
\hline & & & PO \\
\hline 1 & PO-B10-14 & & 1.00 \\
\hline 2 & PO-B10-15 & & 0.88 \\
\hline 3 & PO-B10-16 & & 0.86 \\
\hline 4 & PO-B10-17 & & 0.88 \\
\hline 5 & PO-B20-12 & & 0.91 \\
\hline 6 & PO-B20-13 & & 0.87 \\
\hline \multicolumn{4}{|c|}{ Active Regeneration } \\
\hline & & AR-RMP & $\mathbf{A R}$ \\
\hline 7 & AR-ULSD-1 & 0.89 & 0.76 \\
\hline 8 & AR-ULSD-2 & 0.87 & 0.73 \\
\hline 9 & AR-ULSD-4 & 0.93 & 0.73 \\
\hline 10 & AR-ULSD-5 & 0.87 & 0.76 \\
\hline 11 & AR-ULSD-6 & 0.87 & 0.74 \\
\hline 12 & AR-B10-1 & 0.88 & 0.75 \\
\hline 13 & AR-B10-2 & 0.87 & 0.74 \\
\hline 14 & AR-B10-4 & 0.87 & 0.76 \\
\hline 15 & AR-B20-1 & 0.87 & 0.77 \\
\hline 16 & AR-B20-2 & 0.88 & 0.74 \\
\hline 17 & AR-B20-5 & 0.86 & 0.72 \\
\hline 18 & AR-B20-6 & 0.87 & 0.73 \\
\hline
\end{tabular}

and PM oxidation has started. The average $C F$ during the active regeneration ramp and active regeneration phases are 0.87 and 0.74 , respectively, with a standard deviation of 0.2 and 0.1 , respectively. This shows that the $C F$ likely does not carry an effect of biodiesel blends or CPF inlet temperature, but is affected by unknown variables that cannot be determined with the experimental data that has been measured. 


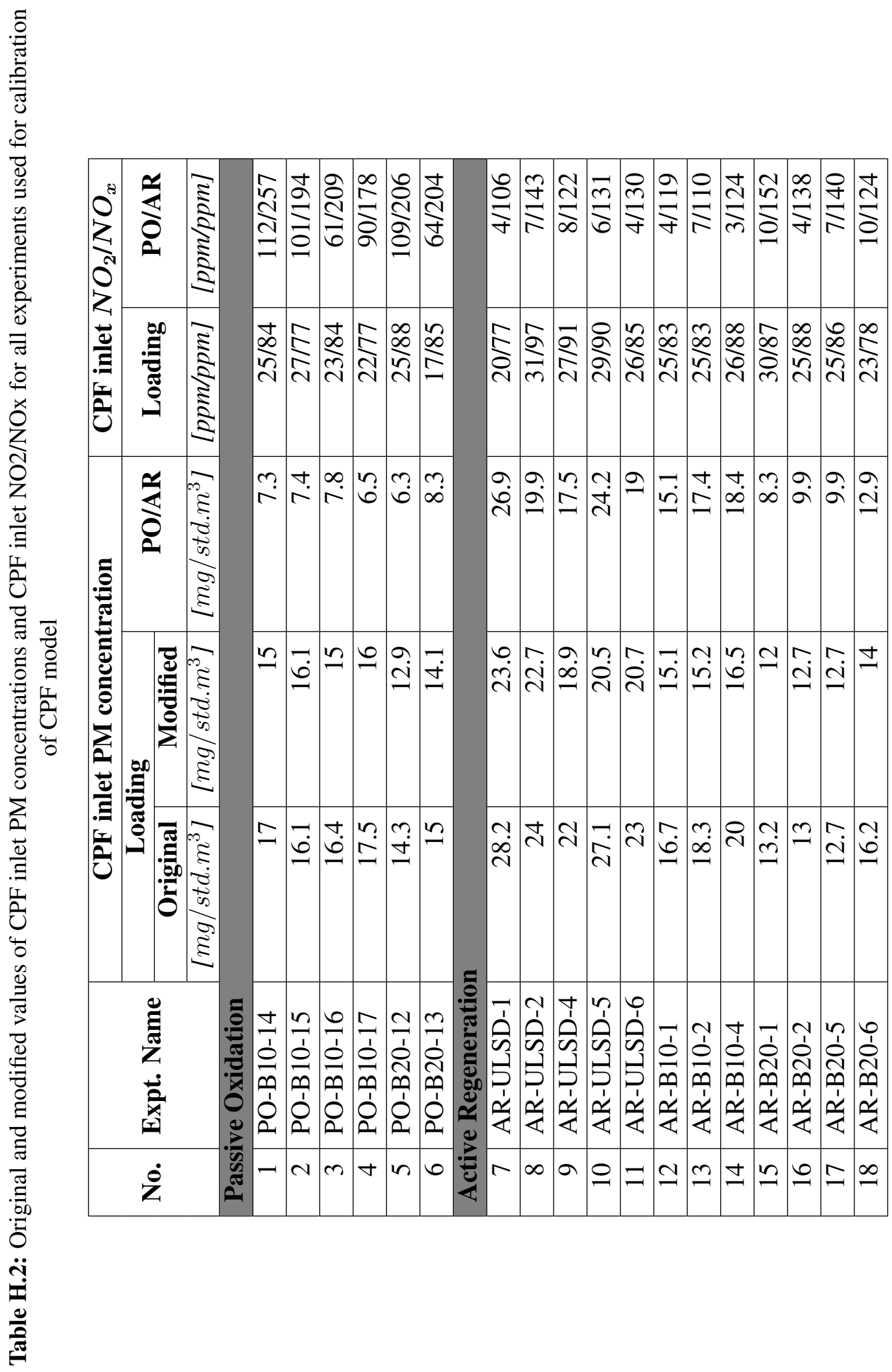




\section{APPEndiX i. Calibration Parameters}
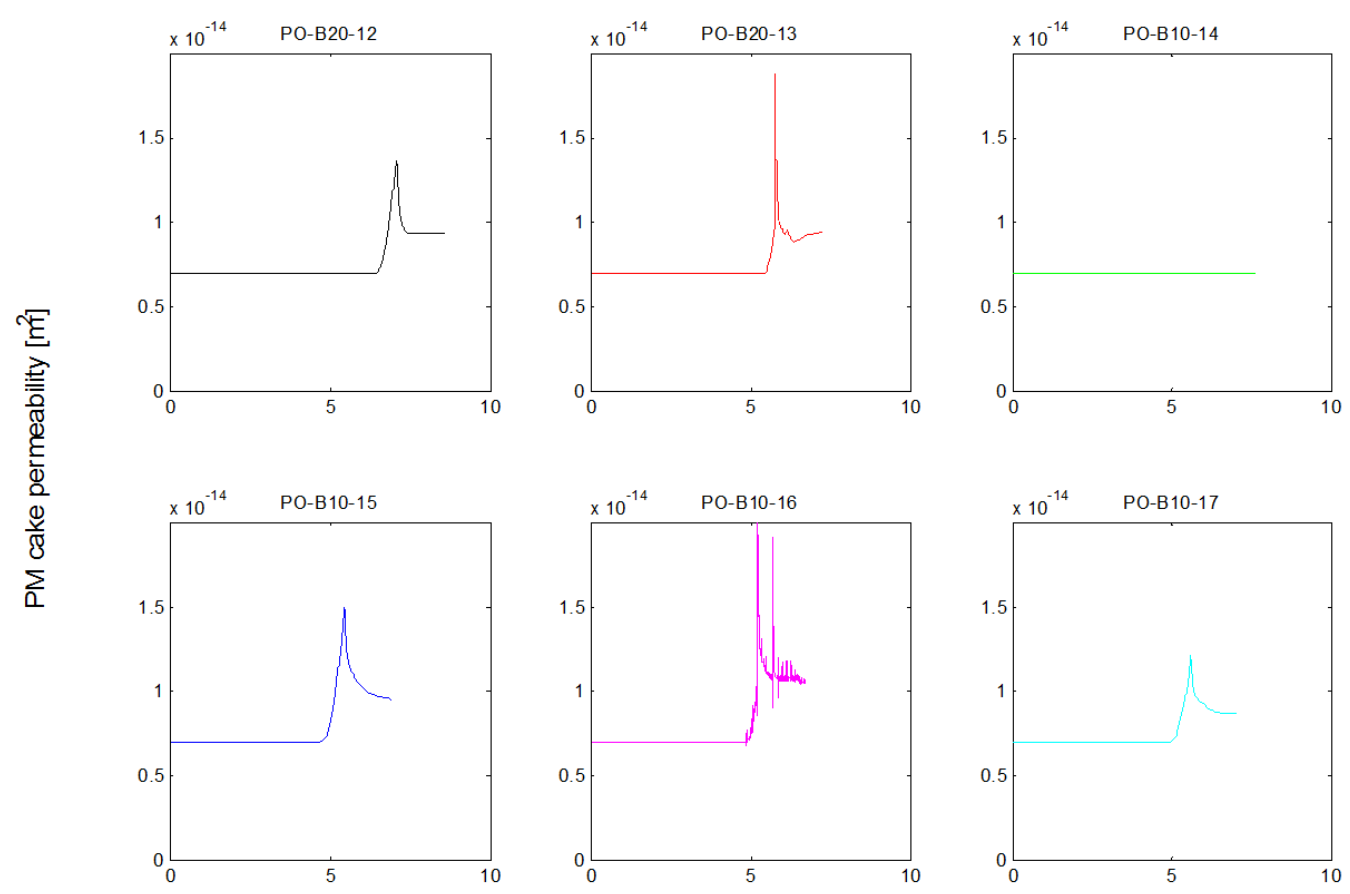

Time $[\mathrm{hr}]$

Figure I.1: PM cake permeability values used for calibration of passive oxidation cases 


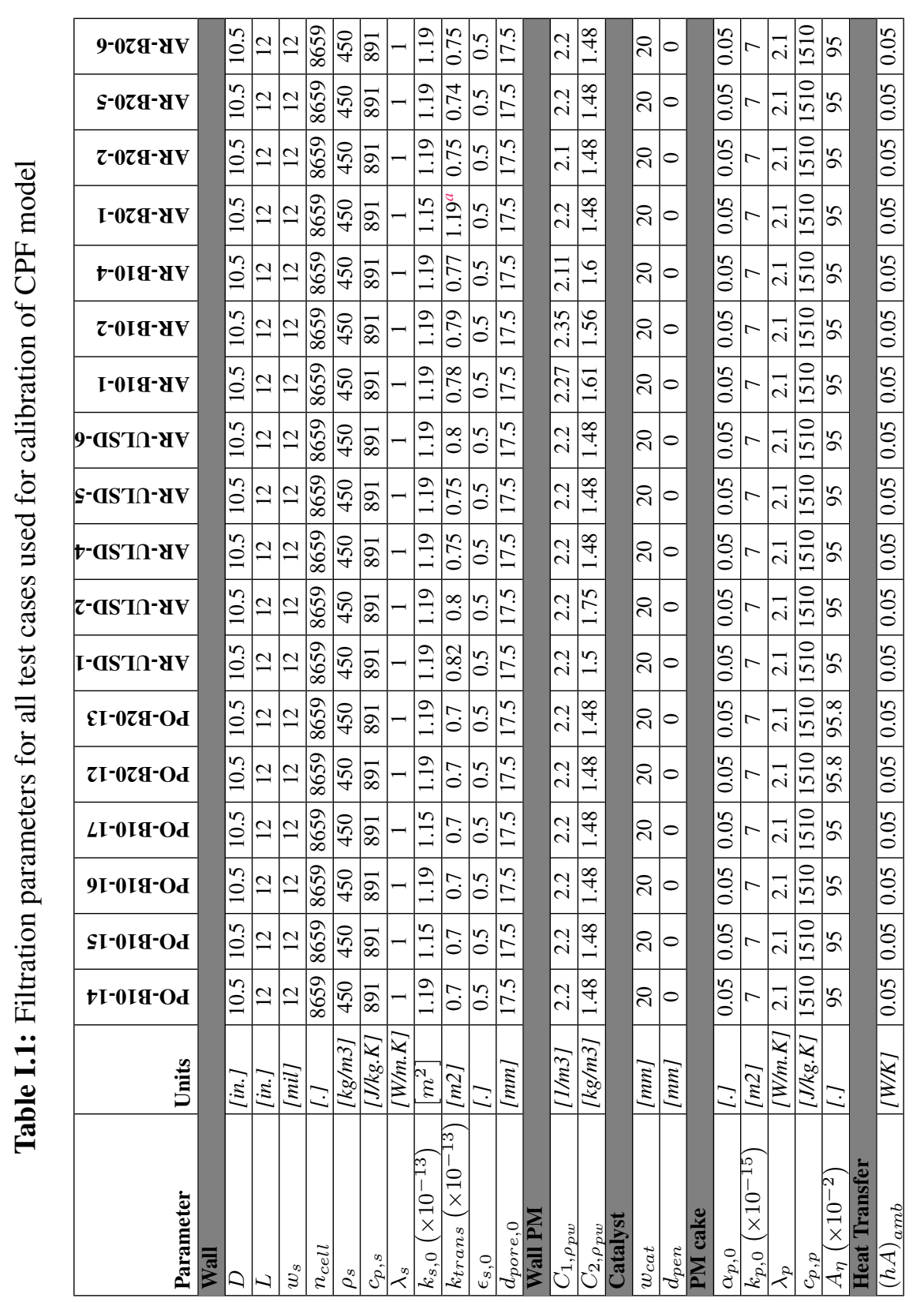

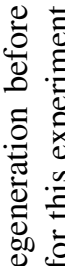

잉

(ช)

영워

ㅎํㅇ.

$\Xi 20$

ฮี

눙흐

:

ปั

$\triangle$ ह 잉

중 홍

응:

․․

홍 폰응

$\exists$ क्ष

on

50 8

음

कo ।

$\Xi \times$

$\stackrel{\Omega}{\rightrightarrows} \stackrel{ }{\hookrightarrow}$

$50 \smile$

긱

홍

$\overparen{\Xi}$

둥

उ

월ㄹ

\&

율

क ज

苛营

焉

Dृ

o $\vec{\Xi}$

है के

卷

चै

$3 F$

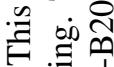

吾选 


\section{APPENDIX J. COMPARISON OF MODEL RESUlTS TO EXPERIMENTAL DATA}

Table J.1: Comparison of experimental and model total pressure drop across the CPF at three different time points during each experiment. Model results are from simulations using filtration parameters in Table I.1, catalytic reaction kinetics from Table 5.2 and PM oxidation kinetics from

Table 5.3

\begin{tabular}{|r|r|r|r|r|r|r|r|r|r|r|}
\hline \multirow{2}{*}{ No. } & \multirow{2}{*}{ Expt. name } & \multicolumn{9}{|c|}{ Pressure Drop $[\mathrm{kPa}]$} \\
\cline { 3 - 11 } & & \multicolumn{3}{|c|}{ End of loading } & \multicolumn{3}{|c|}{ End of PO/AR } & \multicolumn{2}{c|}{ End of post-loading } \\
\cline { 3 - 12 } & & Expt. & Model & Diff. & Expt. & Model & Diff. & Expt. & Model & Diff. \\
\hline 1 & PO-B10-14 & 5.94 & 5.68 & 0.26 & 2.37 & 2.29 & 0.08 & 6.48 & 6.44 & 0.04 \\
\hline 2 & PO-B10-15 & 6.04 & 5.78 & 0.26 & 2.34 & 2.41 & -0.08 & 4.52 & 4.66 & -0.13 \\
\hline 3 & PO-B10-16 & 5.89 & 5.66 & 0.23 & 6.02 & 5.91 & 0.12 & 4.39 & 4.38 & 0.00 \\
\hline 4 & PO-B10-17 & 5.89 & 5.96 & -0.07 & 2.55 & 2.62 & -0.07 & 3.97 & 4.08 & -0.12 \\
\hline 5 & PO-B20-12 & 6.00 & 5.84 & 0.16 & 2.45 & 2.50 & -0.05 & 4.47 & 4.47 & 0.00 \\
\hline 6 & PO-B20-13 & 6.00 & 5.80 & 0.20 & 5.90 & 5.88 & 0.02 & 4.41 & 4.43 & -0.02 \\
\hline 7 & AR-ULSD-1 & 6.35 & 6.21 & 0.13 & 4.04 & 4.11 & -0.07 & 5.15 & 5.21 & -0.06 \\
\hline 8 & AR-ULSD-2 & 6.13 & 6.17 & -0.04 & 4.85 & 5.02 & -0.17 & 4.61 & 4.67 & -0.06 \\
\hline 9 & AR-ULSD-4 & 5.18 & 5.09 & 0.09 & 3.52 & 3.33 & 0.19 & 3.78 & 3.87 & -0.09 \\
\hline 10 & AR-ULSD-5 & 5.95 & 6.03 & -0.07 & 3.60 & 3.59 & 0.02 & 4.88 & 4.84 & 0.04 \\
\hline 11 & AR-ULSD-6 & 6.39 & 6.37 & 0.02 & 3.72 & 3.69 & 0.02 & 4.76 & 4.73 & 0.03 \\
\hline 12 & AR-B10-1 & 6.22 & 6.00 & 0.22 & 3.39 & 3.16 & 0.24 & 4.44 & 4.48 & -0.04 \\
\hline 13 & AR-B10-2 & 6.13 & 5.94 & 0.19 & 3.46 & 3.39 & 0.07 & 4.38 & 4.68 & -0.29 \\
\hline 14 & AR-B10-4 & 6.19 & 5.83 & 0.36 & 3.94 & 3.57 & 0.37 & 4.56 & 4.55 & 0.01 \\
\hline 15 & AR-B20-1 & 5.14 & 5.41 & -0.26 & 3.33 & 3.40 & -0.07 & 4.66 & 4.87 & -0.21 \\
\hline 16 & AR-B20-2 & 6.02 & 5.92 & 0.10 & 3.19 & 3.01 & 0.18 & 4.19 & 4.27 & -0.07 \\
\hline 17 & AR-B20-5 & 5.82 & 5.78 & 0.04 & 3.22 & 3.19 & 0.03 & 4.13 & 4.13 & 0.01 \\
\hline 18 & AR-B20-6 & 6.10 & 5.90 & 0.19 & 3.53 & 3.46 & 0.08 & 4.31 & 4.40 & -0.09 \\
\hline
\end{tabular}




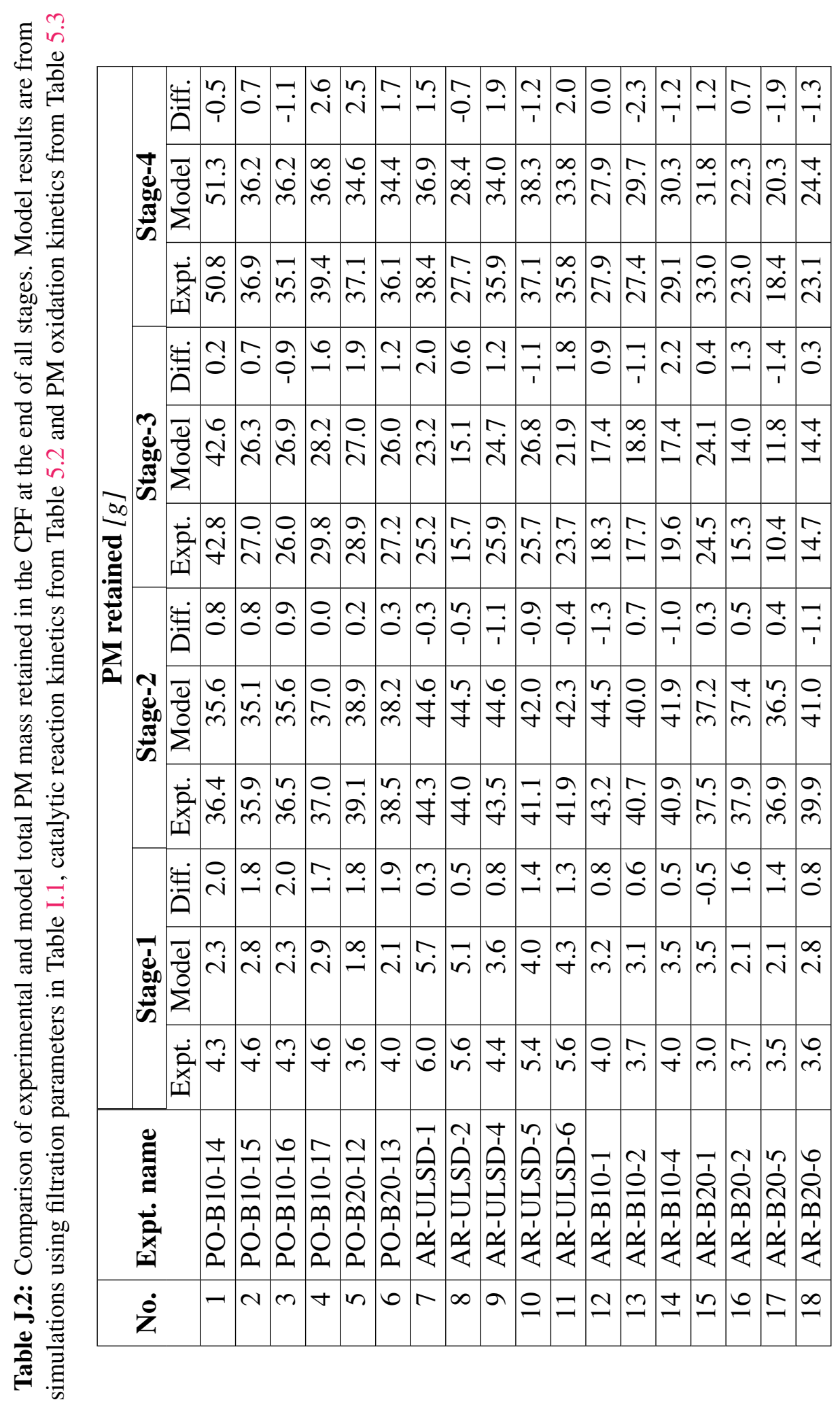


Table J.3: Comparison of experimental and model CPF total filtration efficiency during Stage-2 loading. Model results are from simulations using filtration parameters in Table I.1, catalytic reaction kinetics from Table 5.2 and PM oxidation kinetics from Table 5.3

\begin{tabular}{|r|l|r|r|r|}
\hline \multirow{2}{*}{ No. } & \multirow{2}{*}{ Expt. name } & \multicolumn{3}{|c|}{ Filtration Efficiency [\%] } \\
\cline { 3 - 5 } & & \multicolumn{3}{|c|}{ Stage-2 } \\
\cline { 3 - 5 } & & Expt. & Model & Diff. \\
\hline 1 & PO-B10-14 & 97.0 & 97.4 & -0.4 \\
\hline 2 & PO-B10-15 & 96.6 & 97.4 & -0.8 \\
\hline 3 & PO-B10-16 & 96.6 & 97.4 & -0.8 \\
\hline 4 & PO-B10-17 & 96.6 & 97.4 & -0.8 \\
\hline 5 & PO-B20-12 & 96.6 & 97.6 & -1.0 \\
\hline 6 & PO-B20-13 & 97.0 & 97.8 & -0.8 \\
\hline 7 & AR-ULSD-1 & 97.2 & 97.4 & -0.2 \\
\hline 8 & AR-ULSD-2 & 97.9 & 97.4 & 0.5 \\
\hline 9 & AR-ULSD-4 & 96.8 & 97.5 & -0.7 \\
\hline 10 & AR-ULSD-5 & 95.4 & 97.5 & -2.1 \\
\hline 11 & AR-ULSD-6 & 97.2 & 97.4 & -0.2 \\
\hline 12 & AR-B10-1 & 97.5 & 97.4 & 0.1 \\
\hline 13 & AR-B10-2 & 97.5 & 97.4 & 0.1 \\
\hline 14 & AR-B10-4 & 96.5 & 97.4 & -0.9 \\
\hline 15 & AR-B20-1 & 97.3 & 96.4 & 0.9 \\
\hline 16 & AR-B20-2 & 98.2 & 97.4 & 0.8 \\
\hline 17 & AR-B20-5 & 97.1 & 97.4 & -0.3 \\
\hline 18 & AR-B20-6 & 93.5 & 97.4 & -3.9 \\
\hline
\end{tabular}


Table J.4: Comparison of experimental and model CPF outlet temperature during PO/AR. Model results are from simulations using filtration parameters in Table I.1, catalytic reaction kinetics from Table 5.2 and PM oxidation kinetics from Table 5.3

\begin{tabular}{|r|l|r|r|r|r|}
\hline \multirow{2}{*}{ No. } & \multirow{2}{*}{ Expt. name } & \multicolumn{4}{|c|}{ Average CPF temperature $\left[{ }^{\circ} \mathrm{C}\right]$} \\
\cline { 3 - 6 } & & \multirow{2}{*}{ UPCPF } & \multicolumn{3}{|c|}{ DNCPF } \\
\cline { 3 - 6 } & & & Expt. & Model & Diff. \\
\hline 1 & PO-B10-14 & 253 & 253 & 252 & 1 \\
\hline 2 & PO-B10-15 & 356 & 355 & 353 & 2 \\
\hline 3 & PO-B10-16 & 408 & 407 & 406 & 2 \\
\hline 4 & PO-B10-17 & 356 & 355 & 354 & 1 \\
\hline 5 & PO-B20-12 & 350 & 348 & 348 & 1 \\
\hline 6 & PO-B20-13 & 405 & 404 & 403 & 1 \\
\hline 7 & AR-ULSD-1 & 561 & 569 & 572 & -3 \\
\hline 8 & AR-ULSD-2 & 610 & 624 & 617 & 7 \\
\hline 9 & AR-ULSD-4 & 531 & 535 & 536 & 0 \\
\hline 10 & AR-ULSD-5 & 529 & 536 & 536 & 0 \\
\hline 11 & AR-ULSD-6 & 537 & 546 & 549 & -3 \\
\hline 12 & AR-B10-1 & 529 & 531 & 533 & -2 \\
\hline 13 & AR-B10-2 & 528 & 529 & 528 & 1 \\
\hline 14 & AR-B10-4 & 560 & 564 & 572 & -8 \\
\hline 15 & AR-B20-1 & 478 & 480 & 480 & 0 \\
\hline 16 & AR-B20-2 & 506 & 514 & 513 & 1 \\
\hline 17 & AR-B20-5 & 531 & 538 & 538 & 0 \\
\hline 18 & AR-B20-6 & 535 & 546 & 543 & 3 \\
\hline
\end{tabular}




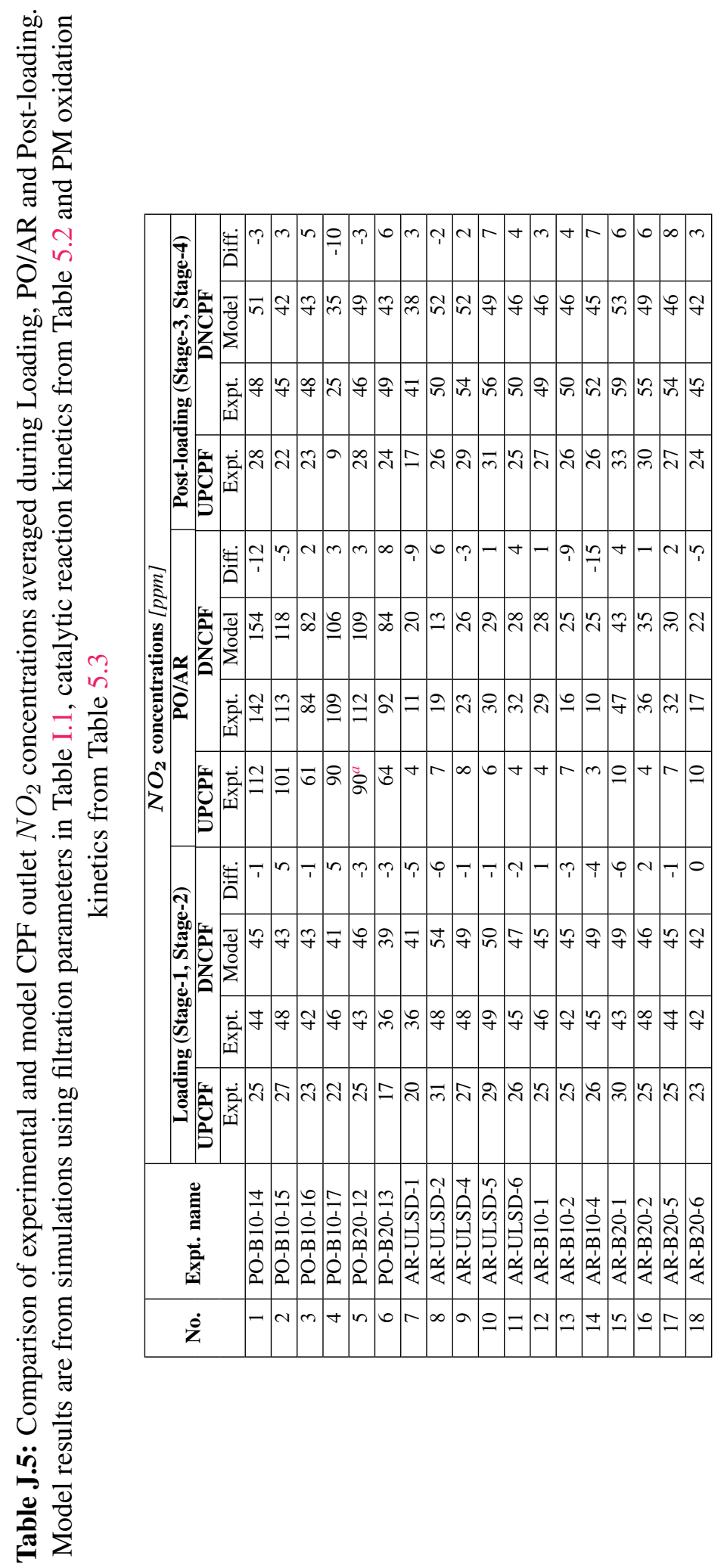

$\because \mathscr{\square}$

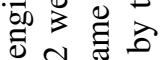

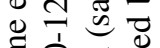

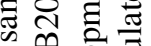

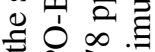

음

के छี 11

๘

䂧

를

广)

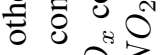

\&OZ

设市

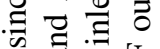

苛它完

Z

๑.

늘

चै ठ ठ

空

ช

D

次 릉

苟

苛

过西

I $0^{*} \pm$

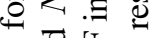

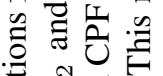

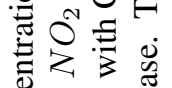

进

응

을

结号。

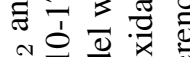

승 훙

之ô่

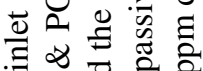

능

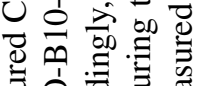

웅

d

ข 0 宁

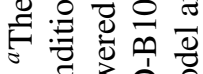




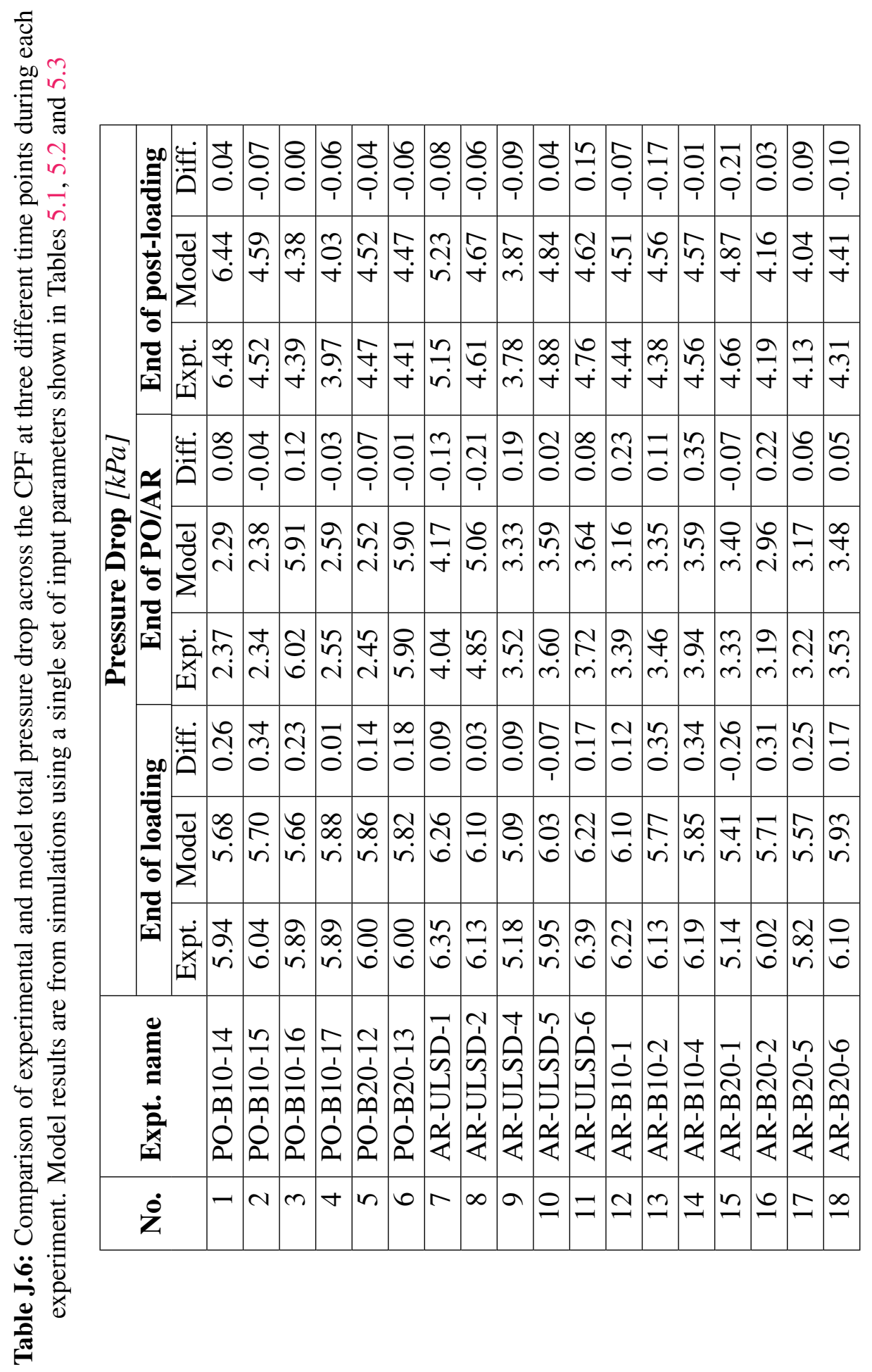




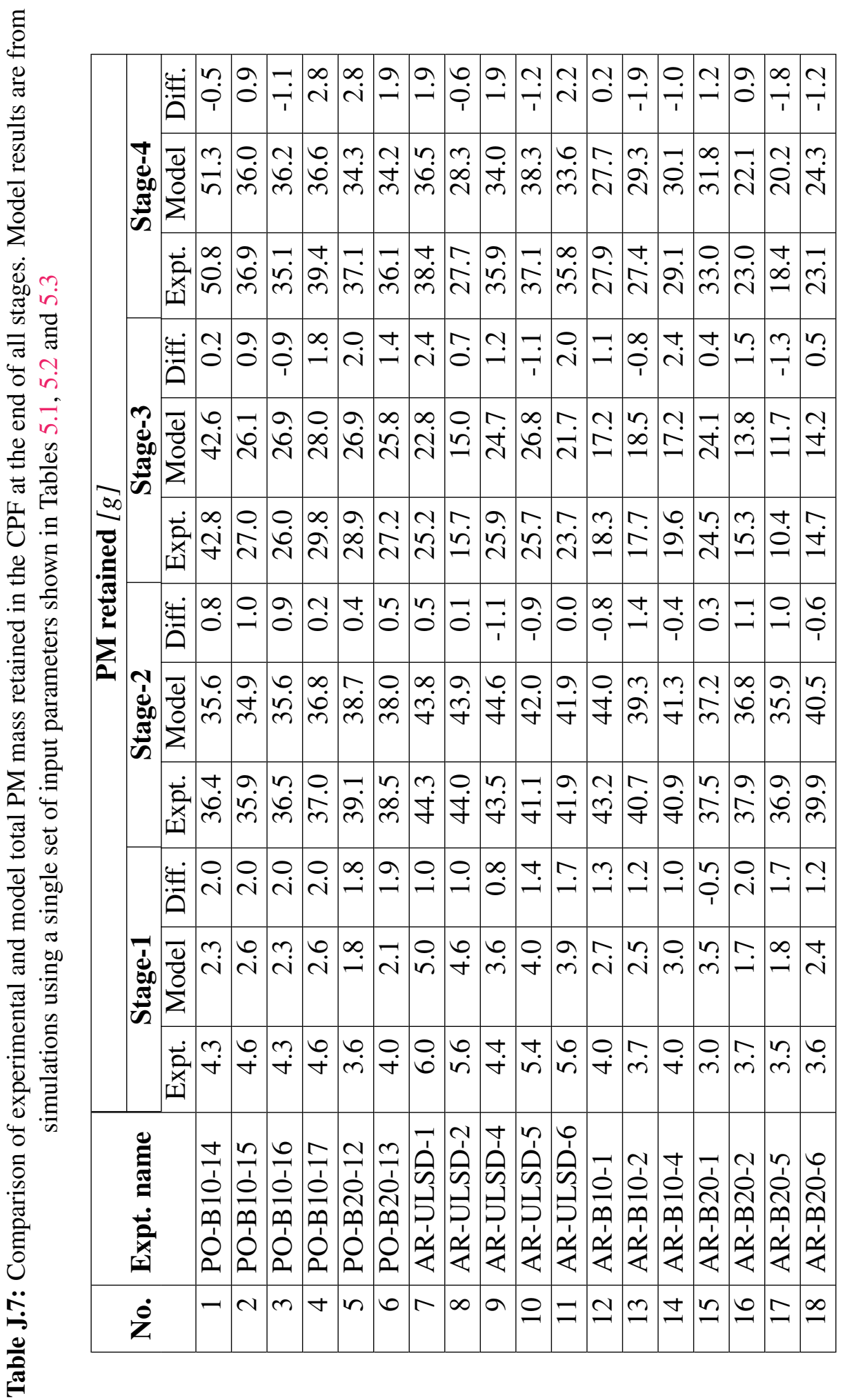


Table J.8: Comparison of experimental and model CPF total filtration efficiency during Stage-2 loading. Model results are from simulations using a single set of input parameters shown in Tables 5.1, 5.2 and 5.3

\begin{tabular}{|r|l|r|r|r|}
\hline \multirow{2}{*}{ No. } & \multirow{2}{*}{ Expt. name } & \multicolumn{3}{|c|}{ Filtration Efficiency [\%] } \\
\cline { 3 - 5 } & & Stage-2 \\
\cline { 3 - 5 } & & Expt. & Model & Diff. \\
\hline 1 & PO-B10-14 & 97.0 & 97.4 & -0.4 \\
\hline 2 & PO-B10-15 & 96.6 & 97.4 & -0.8 \\
\hline 3 & PO-B10-16 & 96.6 & 97.4 & -0.8 \\
\hline 4 & PO-B10-17 & 96.6 & 97.5 & -0.9 \\
\hline 5 & PO-B20-12 & 96.6 & 97.2 & -0.6 \\
\hline 6 & PO-B20-13 & 97.0 & 97.4 & -0.4 \\
\hline 7 & AR-ULSD-1 & 97.2 & 97.5 & -0.3 \\
\hline 8 & AR-ULSD-2 & 97.9 & 97.5 & 0.4 \\
\hline 9 & AR-ULSD-4 & 96.8 & 97.5 & -0.7 \\
\hline 10 & AR-ULSD-5 & 95.4 & 97.5 & -2.1 \\
\hline 11 & AR-ULSD-6 & 97.2 & 97.5 & -0.3 \\
\hline 12 & AR-B10-1 & 97.5 & 97.5 & 0.0 \\
\hline 13 & AR-B10-2 & 97.5 & 97.5 & 0.0 \\
\hline 14 & AR-B10-4 & 96.5 & 97.5 & -1.0 \\
\hline 15 & AR-B20-1 & 97.3 & 96.4 & 0.9 \\
\hline 16 & AR-B20-2 & 98.2 & 97.1 & 1.1 \\
\hline 17 & AR-B20-5 & 97.1 & 97.1 & 0.0 \\
\hline 18 & AR-B20-6 & 93.5 & 97.4 & -3.9 \\
\hline
\end{tabular}




\section{APPENDIX K. AdDitionAL PM REACTION Rate Results From Calibrated PM Oxidation Kinetic PARAmeters for THE THREE Fuels STUdied}

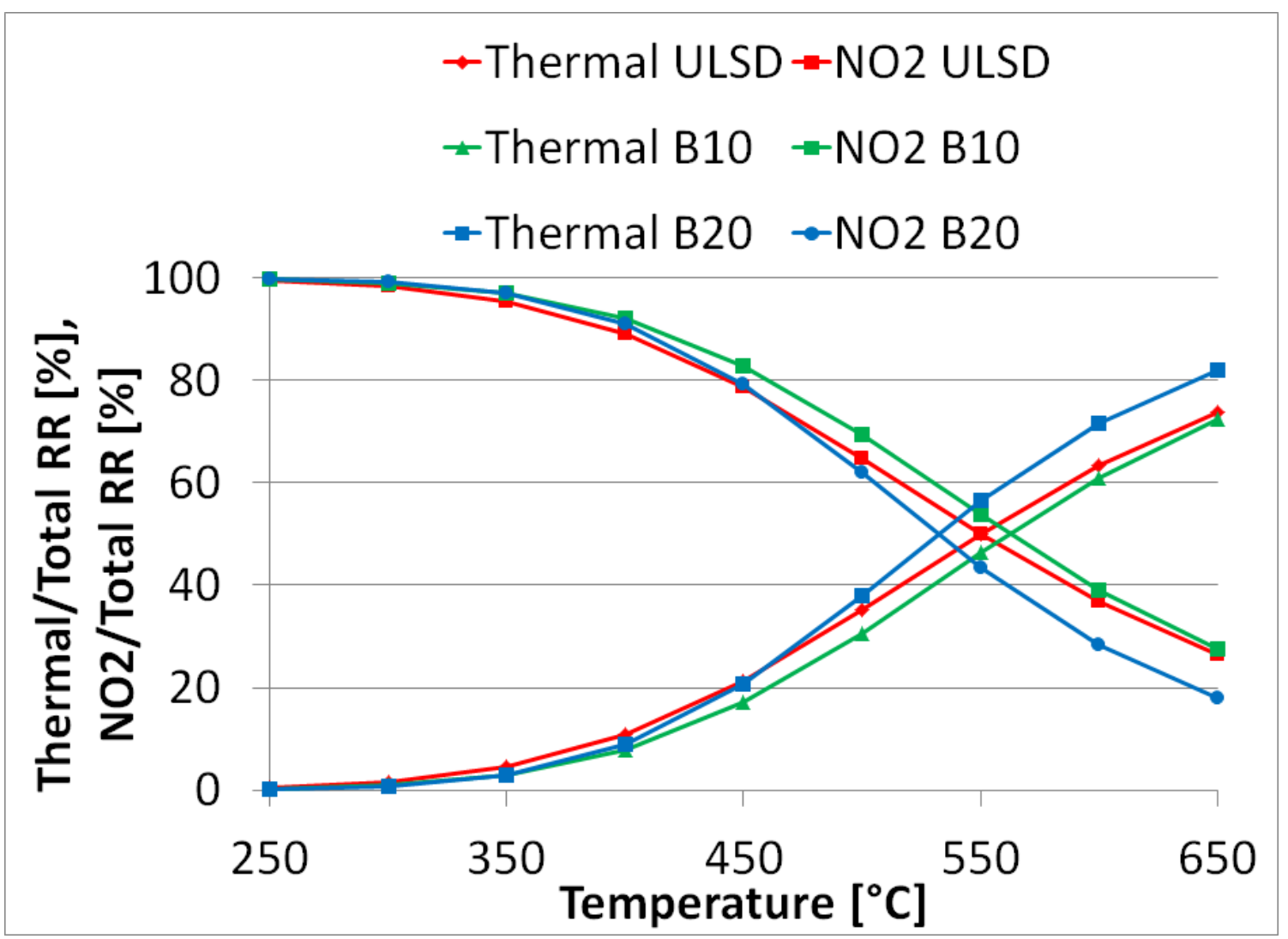

Figure K.1: Thermal to total and $\mathrm{NO}_{2}$-assisted to total reaction rate percentages shown for 3 different fuel types $-\mathrm{O}_{2}=9 \%$ Vol. and $\mathrm{NO}_{2}=50 \mathrm{ppm}$. Results for $\mathrm{O}_{2}=9 \%$ Vol. and $\mathrm{NO}_{2}=100$ ppm and $150 \mathrm{ppm}$ are shown in Table K.1 


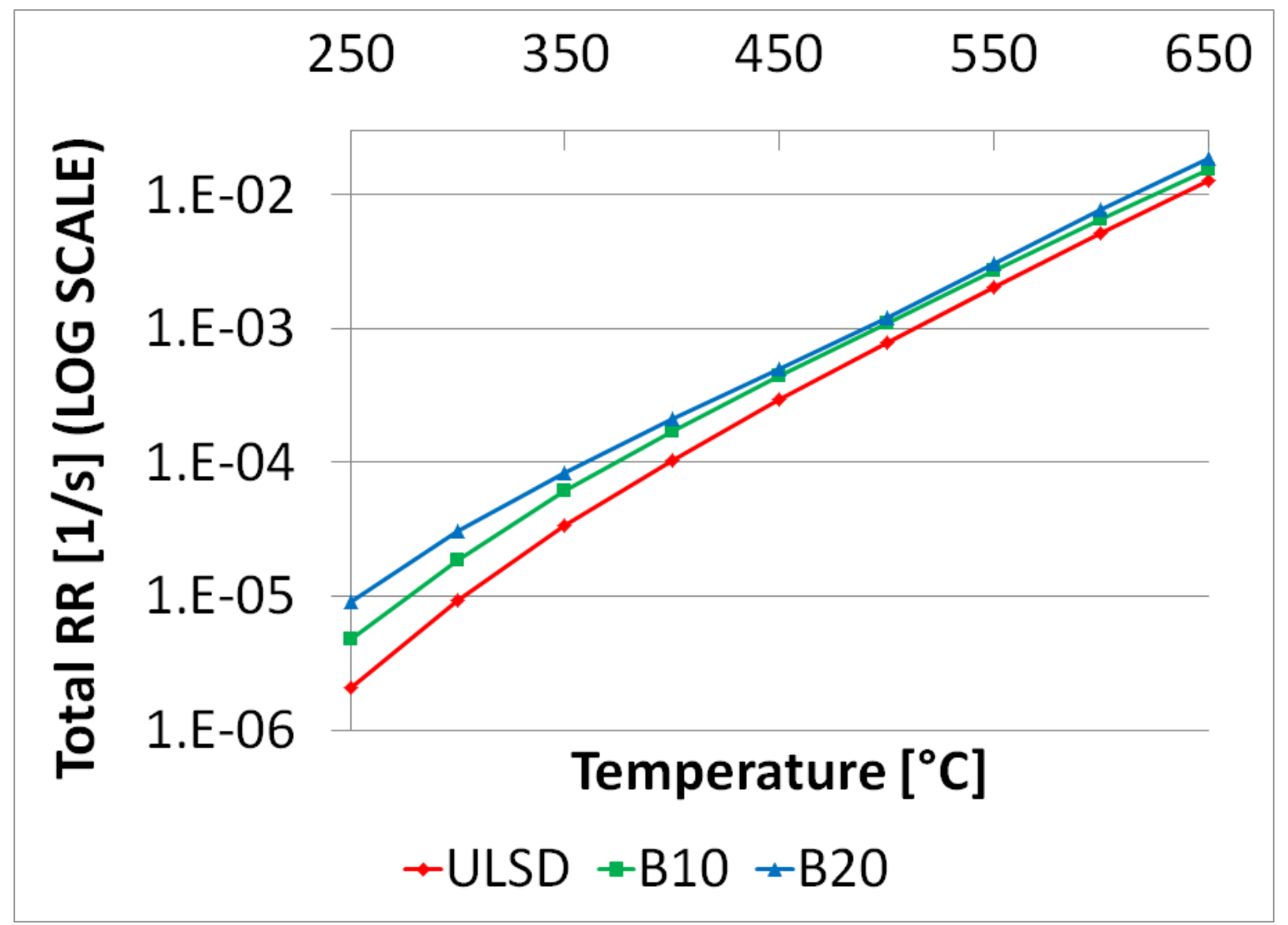

Figure K.2: Total reaction rates along y axis (shown in log-scale) versus temperature for 3 different fuel types $-\mathrm{O}_{2}=9 \%$ Vol. and $\mathrm{NO}_{2}=50 \mathrm{ppm}$. Results for $\mathrm{O}_{2}=9 \%$ Vol. and $\mathrm{NO}_{2}=100$ ppm and 150 ppm are shown in Table K.1 


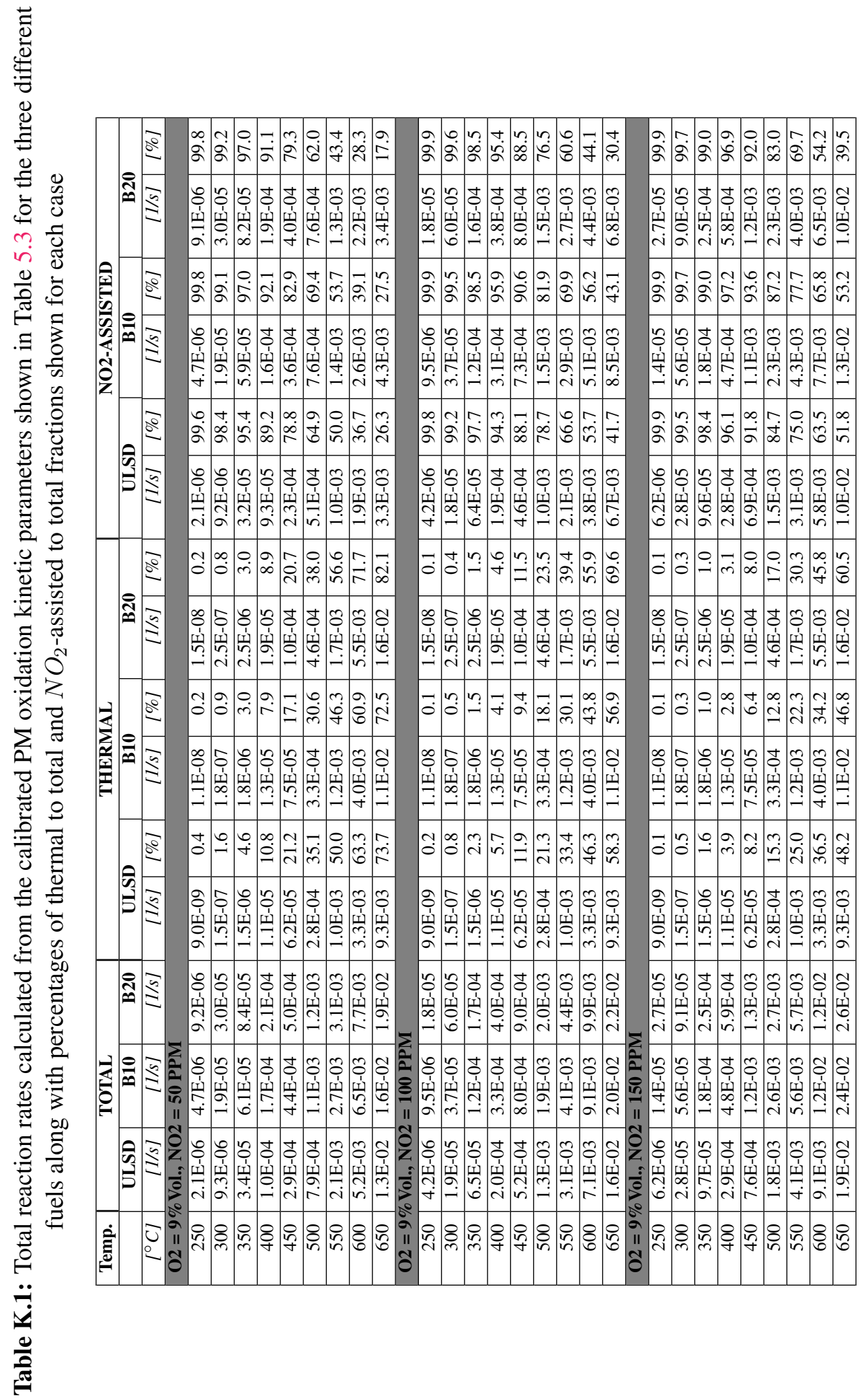




\section{APPENDiX L. COPYRight AgreEmEnTS ANd Letters of Permission}

For Figure 2.4

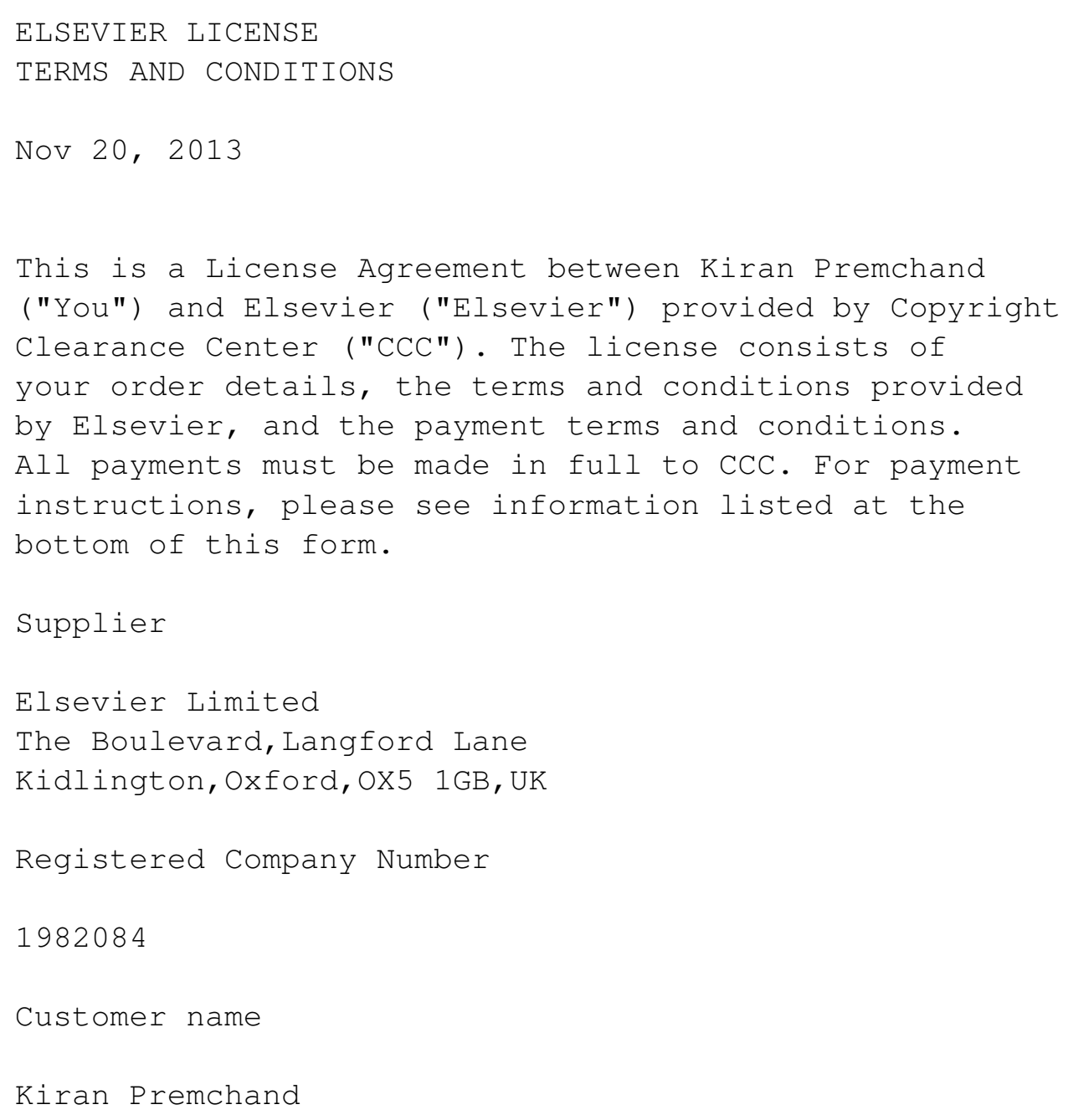




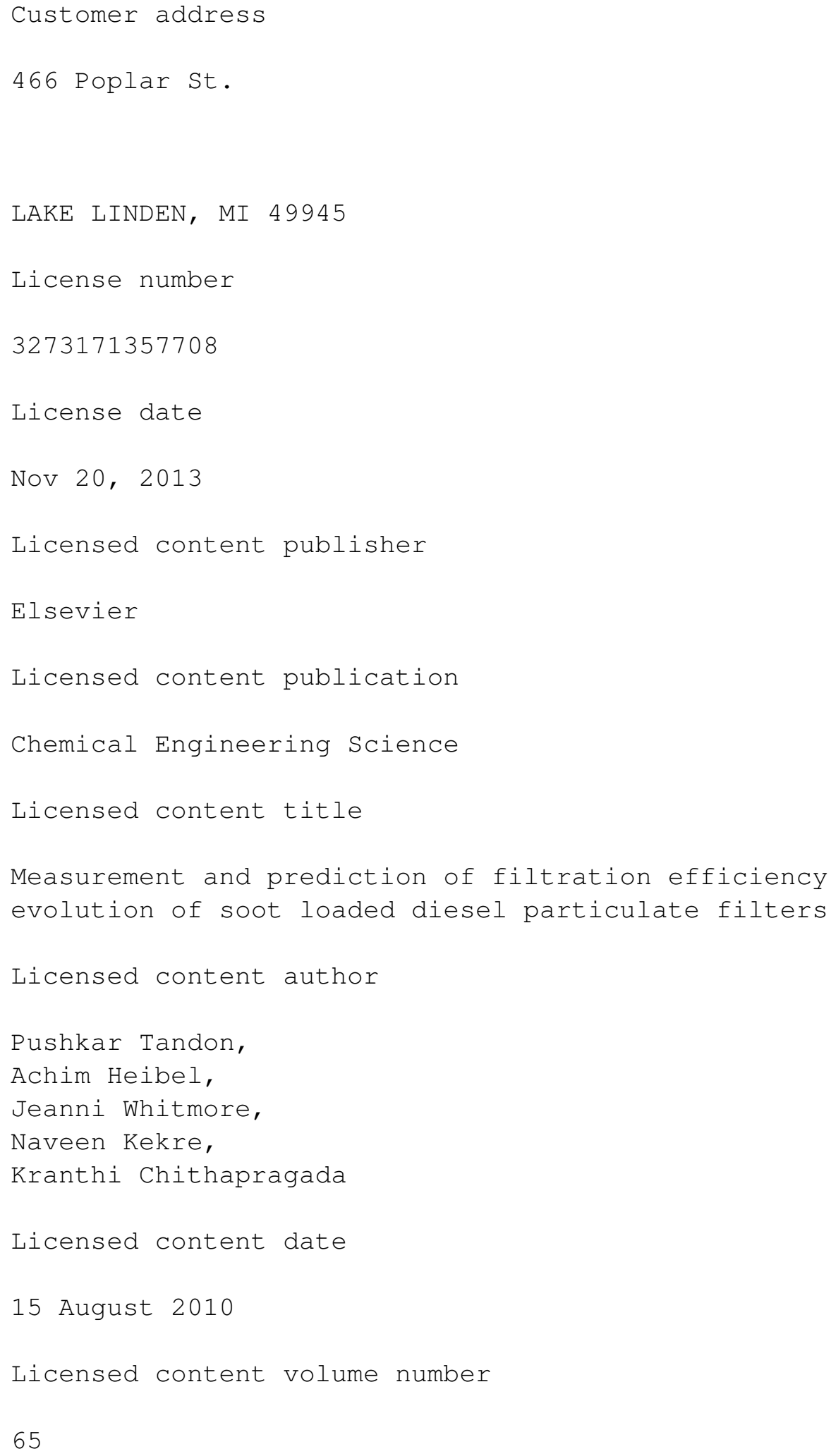


Licensed content issue number

16

Number of pages

10

Start Page

4751

End Page

4760

Type of Use

reuse in a thesis/dissertation

Portion

figures/tables/illustrations

Number of figures/tables/illustrations

1

Format

both print and electronic

Are you the author of this Elsevier article?

No

Will you be translating?

No

Title of your thesis/dissertation

Development of a 1-D Catalyzed Diesel Particulate 
Filter Model Using Engine Experimental Data for Simulation of the Performance and the Oxidation of Particulate Matter and Oxides of Nitrogen during Passive Oxidation and Active Regeneration

Expected completion date

Dec 2013

Estimated size (number of pages)

200

Elsevier VAT number

GB $494 \quad 6272 \quad 12$

Permissions price

$0.00 \mathrm{USD}$

VAT/Local Sales Tax

$0.00 \mathrm{USD} / 0.00 \mathrm{GBP}$

Total

$0.00 \mathrm{USD}$

For Figures 2.1, 2.3, 2.5, 2.6, 2.8 and 2.9

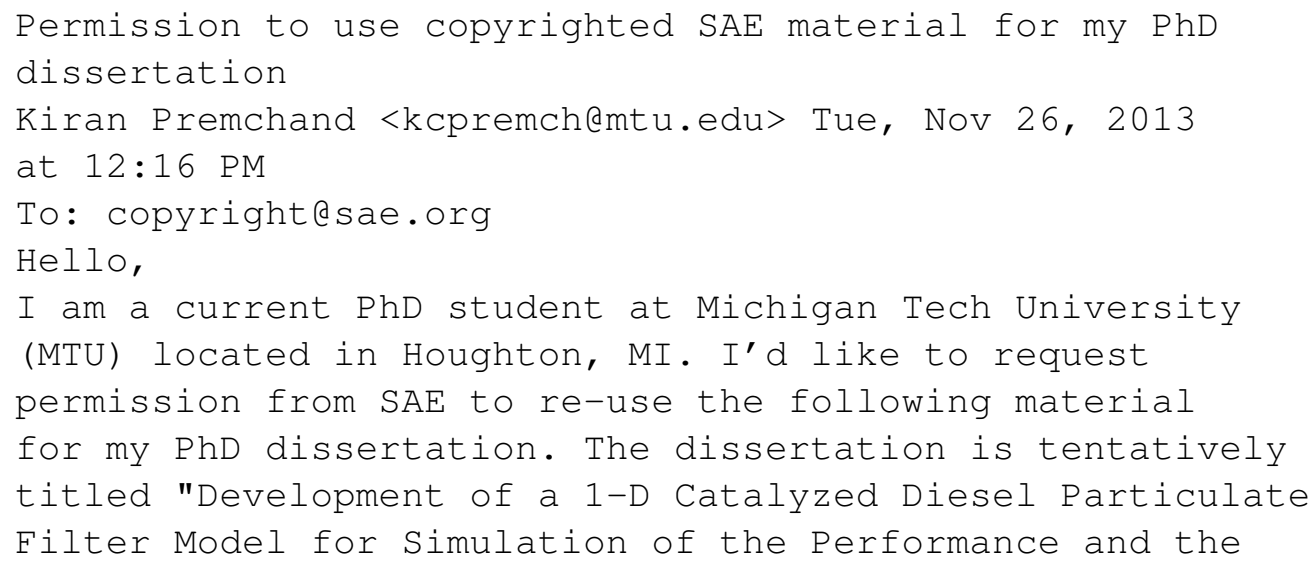


Oxidation of Particulate Matter and Nitrogen Oxides Using Passive Oxidation and Active Regeneration Engine Experimental Data". 7 hard-copies of this dissertation are planned to be distributed between myself, my graduate defense committee members and the library at Michigan Tech. An electronic version is also planned to be submitted to Proquest. The following are the materials that I am requesting permission to use. Please leave this text in the reply. Thanks in advance, Kiran Premchand

1. Figure 3 from the following paper:

Y. Miyairi, S. Miwa, F. Abe, Z. Xu, and Y. Nakasuji, "Numerical Study on Forced Regeneration of Wall-Flow Diesel Particulate Filters", SAE Technical Paper 2001-01-0912, 2001

2. Figure 4 from the following paper:

E. Ohara, Y. Mizuno, Y. Miyairi, T. Mizutani, K. Yuuki, Y. Noguchi, T. Hiramatsu, M. Makino, A. Takahashi, H. Sakai, M. Tanaka, A. Martin, S. Fujii, P. Busch, T. Toyoshima, T. Ito, I. Lappas, and C. D. Vogt, "Filtration Behavior of Diesel Particulate Filters (1)", SAE Technical Paper 2007-01-0921, 2007

3. Figure 7 from the following paper:Z. G. Liu and R. K. Miller "Flow Distributions and Pressure Drops of Wall-Flow Diesel Particulate Filters", SAE Technical Paper 2002-01-1311, 2002

4. Figure 6 from the following paper:

F. Piscaglia, C. J. Rutland, and D. E. Foster, "Development of a CFD Model to Study the Hydrodynamic Characteristics and the Soot Deposition Mechanism on the Porous Wall of a Diesel Particulate Filter", SAE Technical Paper 2005-01-0963, 2005

5. Figure 5 from the following paper:

A. Strzelec, T. Toops, C. S. Daw, D. Foster, and C. Rutland, "Diesel Particulate Oxidation Model: Combined Effects of Volatiles and Fixed Carbon Combustion", SAE Technical Paper 2010-01-2127, 2010

6. Figure $8(a)$ and $8(b)$ from the following paper:

O. A. Haralampous, G. C. Koltsakis, Z. C. Samaras, C. D. Vogt, E. Ohara, Y. Watanabe, and T. Mizutani, "Reaction and Diffusion 
Phenomena in Catalyzed Diesel Particulate Filters", SAE

Technical Paper 2004-01-0696, 2004

Thanks in advance,

Kiran Premchand

Kiran Premchand

Graduate Student

Mechanical Engineering - Engineering Mechanics Dept.

Michigan Technological University

1400 Townsend Drive

Houghton, MI - 49931

Tel: 906-370-0586Fax: 906-487-2822

Terri Kelly <terriesae.org> Mon, Dec 9, 2013 at 2:41 PM

To: Kiran Premchand <kcpremch@mtu.edu>

Dear Kiran,

Thank you for your correspondence requesting permission

to reprint the material below from various SAE papers

in your PhD dissertation (title below) at Michigan

Technological University:

Figure 3 from paper 2001-01-00912

Figure 4 from paper 2007-01-0921

Figure 7 from paper 2002-01-1311

Figure 6 from paper 2005-01-0963

Figure 8(a) and 8(b) from paper 2004-01-0696

Permission is hereby granted, and subject to the following conditions:

Permission is for this one time single use. New requests are required for further use or distribution of the SAE material. The following credit statement must appear below the figures: 'Reprinted with permission from SAE Paper XXXXX* lcopyright 200X** SAE International.'' (*please insert the paper number and **year of publication) We also request that you credit the original source (author, paper number and SAE) in the reference section of your publication. Permission does not cover any third party copyrighted work which may appear in the material requested. Permission must be obtained from the original source.

SAE does not hold the copyright on your 5th paper below (2010-01-2127), therefore, we cannot grant this permission. 
This paper is documented as a government paper. You can contact the paper authors to obtain permission to use this material.

Please feel free to contact me if you need further assistance, and good luck with your dissertation!

Best regards,

Terri Kelly

Intellectual Property Rights Administrator

SAE INTERNATIONAL

400 Commonwealth Drive

Warrendale, PA 15096

$0+1.724 .772 .4095$

f $+1.724-776-9765$

e terriesae.org

www.sae.org

\section{For Figure 2.7}

From: Kiran Premchand <kcpremch@mtu.edu>

To: <andrea.strzelecepnl.gov>

Cc: <astrzelecetamu.edu>

Date: Tue, 10 Dec 2013 09:38:24 -0500

Subject: Fwd: Permission to use copyrighted SAE material

for my PhD dissertation

Hello Dr. Strzelec,

I request permission to re-use figure 5 from SAE paper

2010-01-2127 in my PhD dissertation. I was informed by

SAE that SAE does not hold copyrights to this work

(please see attached message from SAE about this).

Thanks in advance,

Kiran Premchand

Kiran Premchand

Graduate Student

Mechanical Engineering - Engineering Mechanics Dept.

Michigan Technological University

1400 Townsend Drive

Houghton, MI - 49931

Tel: 906-370-0586

Fax: 906-487-2822 
Andrea Strzelec <astrzelec@tamu.edu>

To: Kiran Premchand <kcpremch@mtu.edu>

Tue, Dec 10, 2013 at 11:03 AM

Hi Kiran-

You can absolutely use it. The reason that SAE doesn't hold the copyright is that on all government sponsored work (as mine was by DOE) - the government retains the copyright - so that they can allow unrestricted fair use. So any paper by ORNL, PNNL, SNL, LLNL, etc - you can feel free to use and cite.

Best,

Andrea

\section{For Figures 3.1, 3.3 and E.1, Appendices G and B and Section 3.4.7}

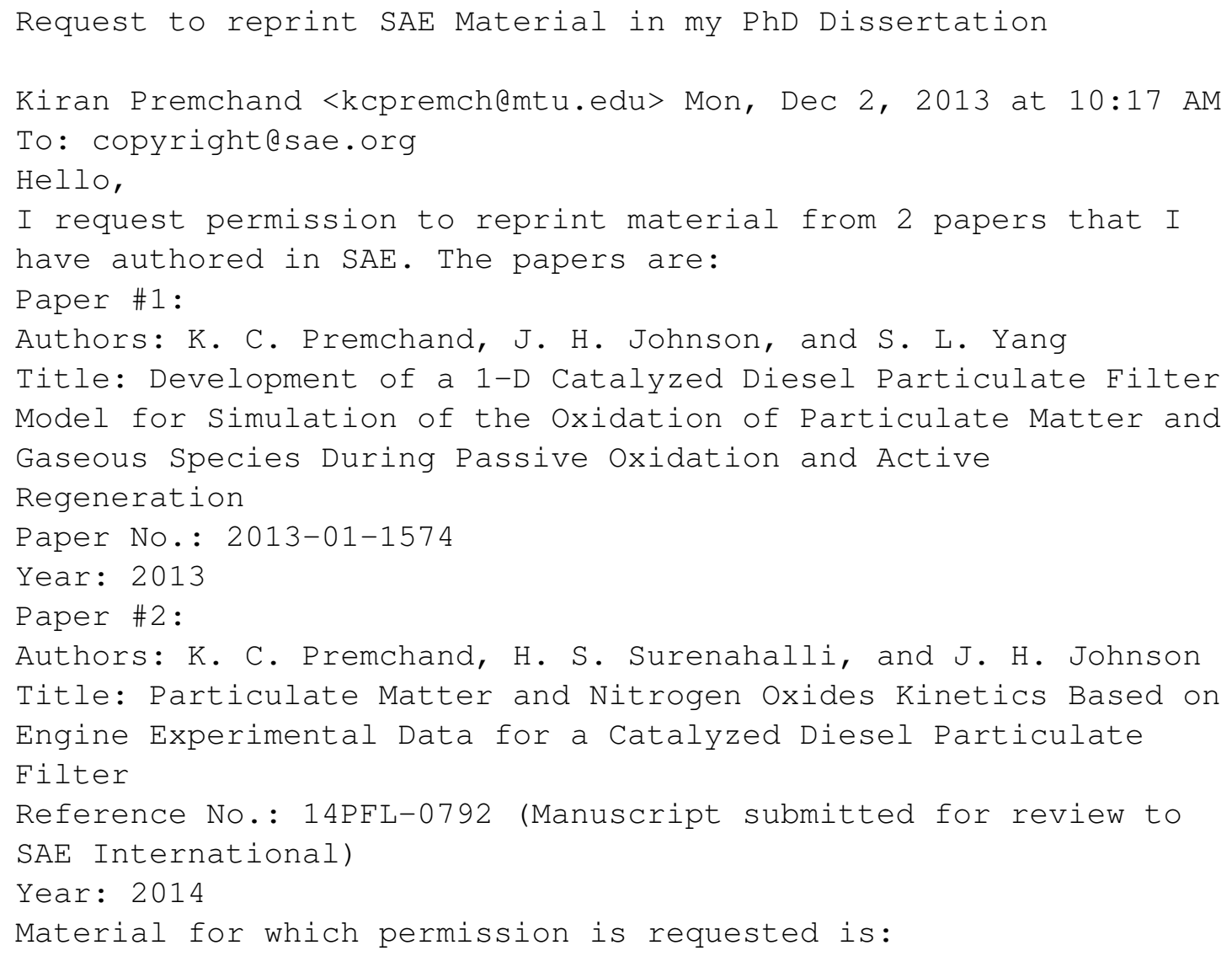




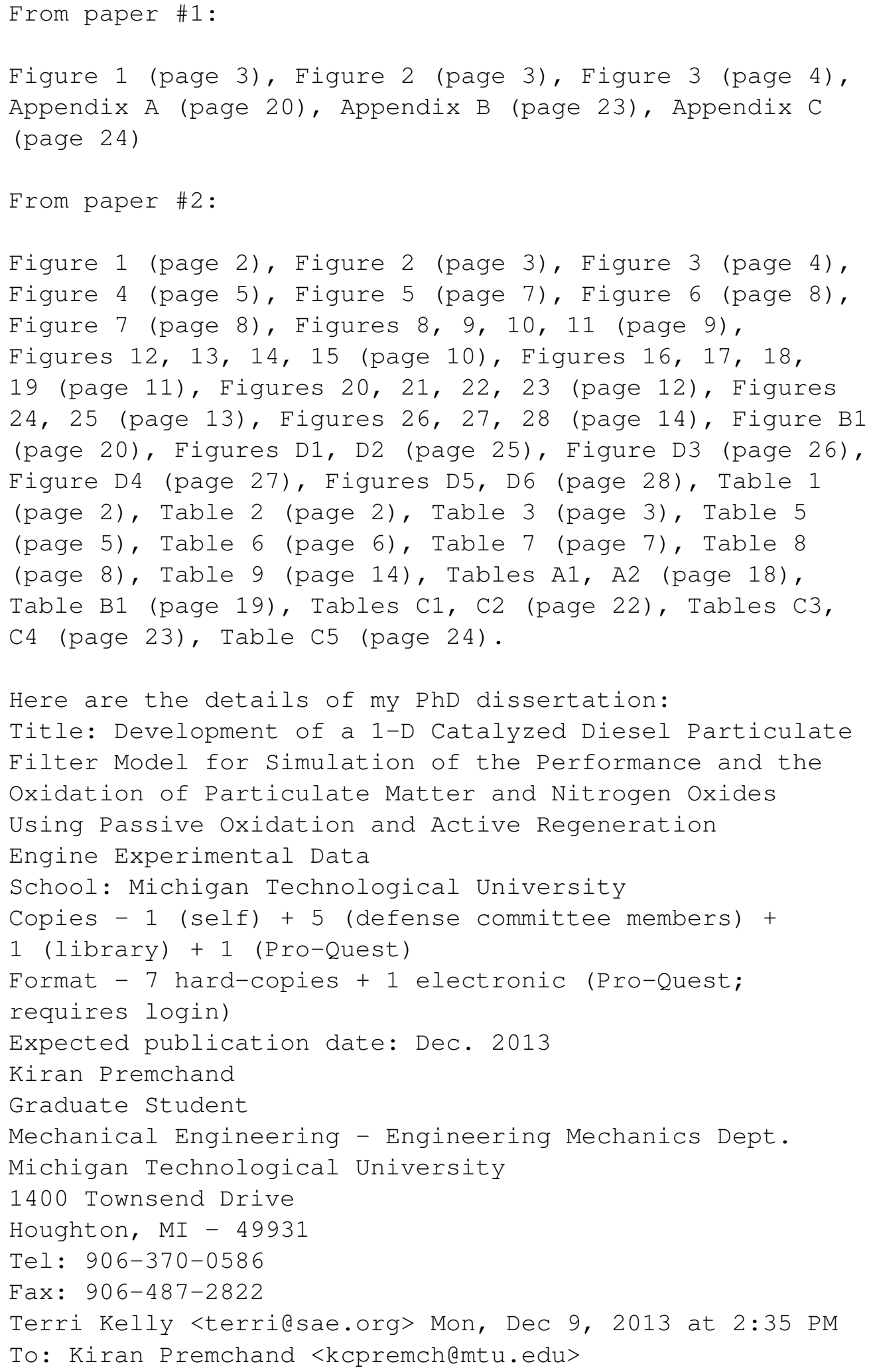




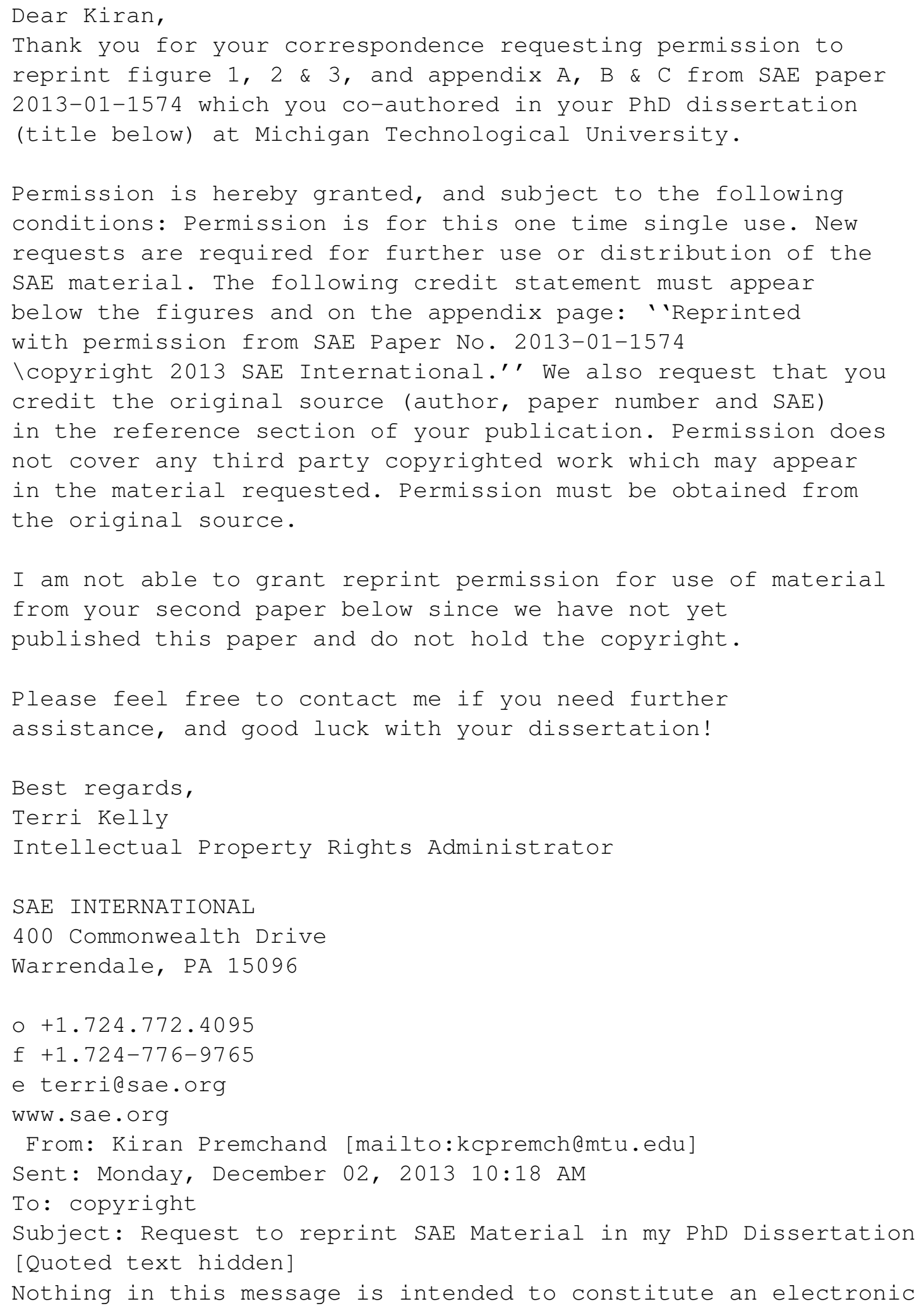


signature unless a specific statement to the contrary is included in this message. Confidentiality Note: This message is intended only for the person or entity to which it is addressed. It may contain confidential and/or proprietary material. Any review, transmission, dissemination or other use, or taking of any action in reliance upon this message by persons or entities other than the intended recipient is prohibited. If you received this message in error, please contact the sender and delete it from your computer.

\section{For Figures 5.33, 5.34 and 5.35}

Kiran,

You have my permission to re-produce the data in the listed figures and any other data that is required from my MS thesis.

Regards, Ryan

On Wed, Nov 20, 2013 at 1:14 PM, Kiran Premchand <kcpremch@mtu.edu> wrote:

Hi Ryan,

May I have permission to re-produce part of the experimental data presented in Figures 6.24, 6.30 and 6.36 of your MS thesis in my PhD dissertation? Please leave this text in your reply.

Thanks in advance, Kiran

Kiran Premchand Graduate Student Mechanical Engineering - Engineering Mechanics Dept. Michigan Technological University 1400 Townsend Drive Houghton, MI - 49931

Tel: 906-370-0586

Fax: 906-487-2822 
Ryan Foley

Graduate Research Assistant

Mechanical Engineering

Michigan Technological University

Office: MEEM Room B009

Lab: (906) 487-3638

Cell: (701) 833-3633

Email: rkfoley@mtu.edu

\section{For Figure 4.1}

Kenneth Shiel <klshiel@mtu.edu> Sat, Dec 14, 2013

at $10: 36 \mathrm{PM}$

To: Kiran Premchand <kcpremch@mtu.edu>

Kiran,

You have my permission to use any material from my thesis you may need. Rock on brother.

Ken

Kiran Premchand <kcpremchemtu.edu>

Permission to re-produce material in my PhD dissertation

Kiran Premchand <kcpremch@mtu.edu> Sat, Dec 14, 2013

at $10: 18 \mathrm{PM}$

To: Kenneth Shiel <klshielemtu.edu>

Hi Ken,

May I have permission to re-produce Figure 3.7 from

your MS Thesis in my PhD dissertation? Please leave

this text in your reply.

Thanks in advance,

Kiran

Kiran Premchand

Graduate Student

Mechanical Engineering - Engineering Mechanics Dept.

Michigan Technological University

1400 Townsend Drive

Houghton, MI - 49931

Tel: $906-370-0586$

Fax: $906-487-2822$ 


\section{For Figure 4.2 and part of Appendix $H$}

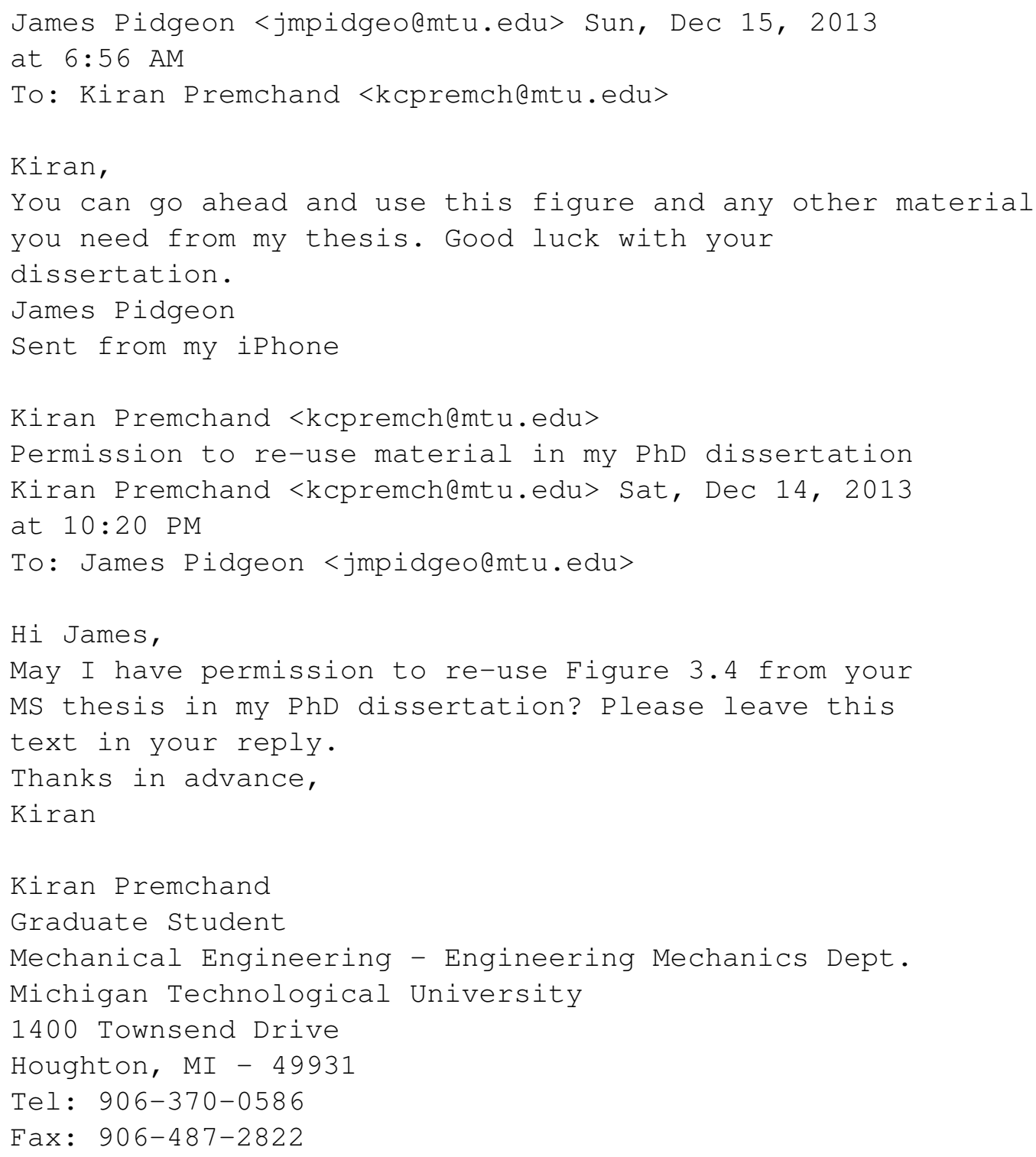

